\title{
The early Development of the Germline in
}

\section{Drosophila melanogaster}

\author{
Dissertation \\ for the award of the degree \\ Doctor rerum naturalium (Dr. rer. nat.) \\ from the Georg-August-Universtät Göttingen \\ within the doctoral program of Biology \\ of the Georg-August-University School of Science
}

submitted by

Andres Hertel

from Strausberg

Göttingen 2018 
Thesis Committee:

PD Dr. Gerd Vorbrüggen, Abteilung Entwicklungsbiologie, Universität Göttingen Prof. Dr. Ernst Wimmer, Abteilung Entwicklungsbiologie, Universität Göttingen Prof. Dr. Reinhard Schuh, RG Molecular Organogenesis, MPIbpc

Members of the examination board:

Reviewer

PD Dr. Gerd Vorbrüggen, Abteilung Entwicklungsbiologie, Universität Göttingen

Second reviewer

Prof. Dr. Ernst Wimmer, Abteilung Entwicklungsbiologie, Universität Göttingen

Further members of the examination board:

Prof. Dr. Reinhard Schuh, RG Molecular Organogenesis, MPIbpc

Prof. Dr. Gregor Bucher, Evolutionary Developmental Genetics, Universität Göttingen

Prof. Dr. Michael Kessel, RG Developmental Biology, MPIbpc

PD Dr. Roland Dosch, Entwicklungsbiochemie, Universitätsmedizin Göttingen

Date of oral examination:

13.02.2018 
ex ovo omnia 



\section{Acknowledgments}

Diverse Leute haben auf die eine oder andere Weise zum Gelingen dieser Arbeit beigetragen. Mein erster Dank gilt meinem Betreuungskommittee Dr. Gerd Vorbrüggen, Prof. Ernst Wimmer und Prof. Reinhard Schuh für das kritische Begleiten der Arbeit während der gesamten Zeit. Insbesondere danke ich Gerd für die unzähligen fruchtbaren Diskussionen und Vorschläge.

Mein zweiter erster Dank gilt Prof. Herbert Jäckle für die Möglichkeit, meine Doktorarbeit in seiner Abteilung anfertigen zu können und für die Finanzierung meiner Stelle über die übliche Zeit hinaus. Eben jene Abteilung bot ein hervorragendes Arbeitsklima mit super Ausstattung und Service. Ich werde in Zukunft sicherlich den Luxus vermissen, mich nicht um Fliegenfutter oder Agarplatten kümmern zu müssen. Für das und weitere Annehmlichkeiten geht ein herzlicher Dank an Ulrike Borchert, Karin Hartwig, Claudia Koch und Regina Krügener! Ohne Euch würde vieles in der Abteilung nicht so funktionieren.

Vielen Dank auch an Tomma Eisbein für das gelegentliche Genotypisieren von Fliegen und das Herstellen der besten RNA in-situ Sonden der Welt und an Dr. Ralf Pflanz, Herr über die Antikörper und die Mikroskope, für Tipps und Diskussionen bezüglich Färbungen und Mikroskopie.

Ninett Wolfram gebührt besonderer Dank für ihre Arbeit am echinoid-Projekt während ihrer Bachelorzeit und für ihre Dienste als Hiwi in den Jahren danach. Auch meinen anderen Hiwis und Praktikanten Nele Vollersen, Folke Ebert, Saskia Köhn, Nadia Prayitno und Carla Perez sei gedankt für tatkräftige Hilfe beim Fliegen flippen, Jungfrauen sammeln oder Embryos fixieren.

Die gesamte Abteilung Jäckle, mit allen vergangenen und rezenten Mitgliedern, hat zu einer sehr angenehme Atmosphäre beigetragen, insbesondere die regelmäßigen Grillund Filmabende mit Ralf, Philip, Eva und Reinhard.

Ein großer Dank von Herzen geht an meine Mutter und Mirko, für die nimmerendende Unterstützung in all den Jahren, motivierende Gespräche und Statistikberatung, und einfach fürs Familie-Sein! 
Wen-ti, thank you for being such an awesome girlfriend! For the company, for traveling, for good food, for countless hours of fun and for just being there. :*

Vielen Dank auch auch Krissy, ohne die ich weit weniger Restaurants in Göttingen kennen würde, für die jahrelange Freundschaft und lustige und interessante Diskussionen. Dann sind da noch die anderen Leute und Dinge, die das Leben in Göttingen (und anderswo) angenehm machten und machen, zum Beispiel Flo, Olli, Philip und Julia, unsere coole Truppe internationaler Metalheads in Göttingen und die Monster Bar. Danke!

Zuletzt sei auch den vielen hier nicht namentlich Erwähnten gedankt, die alle irgendwie ihren Anteil an meinem Leben in den letzten Jahren hatten. 


\section{Contents}

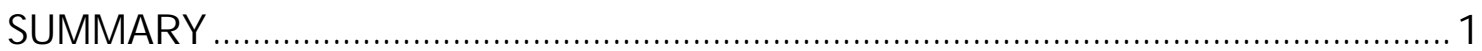

INTRODUCTION ...............................................................................................................................5

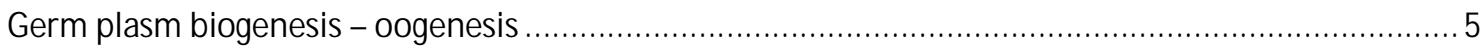

Early embryogenesis

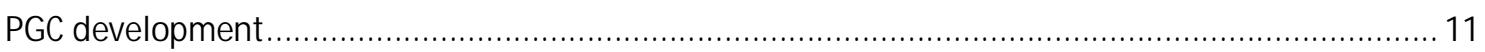

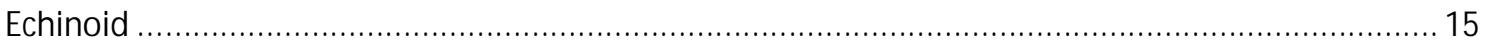

Lost

RESULTS..........................................................................................................................................................21

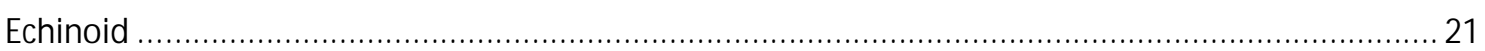

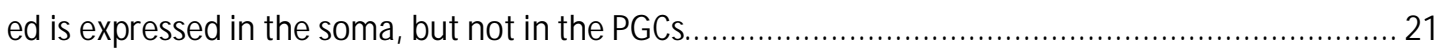

Differential expression of ed prevents the PGCs from transmigrating ........................................ 22

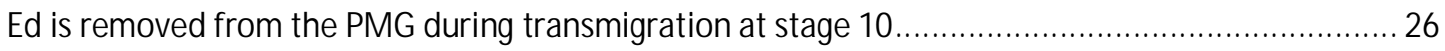

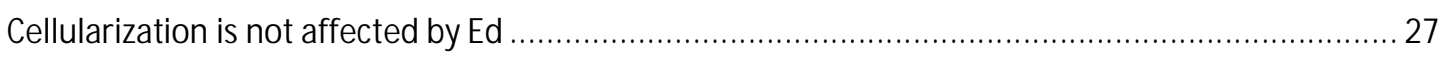

An apical actomyosin network depends on the differential expression of Ed .................................. 29

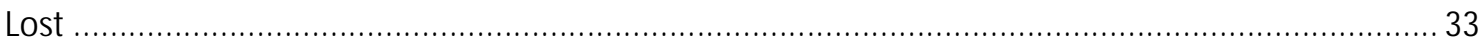

Lost is a sponge body component and ubiquitously present in early embryos ................................. 33

PGC development is impaired in maternal lost mutant embryos .................................................. 34

Cellularization defects at posterior pole in embryos lacking maternal lost...................................... 38

The posterior somatic nuclei have less transcription in maternal lost mutants................................. 41

Fluorescent in-situ hybridyzation reveals localization patterns of germ plasm RNAs ....................... 43

Comparing germ plasm RNA staining patterns using FISH intensity profiles.................................. 45

The pgcFISH intensity profile is altered in maternal lostembryos ................................................... 47

Maternal lost mutants combine features of pgc LOF and pgcGOF ................................................ 49

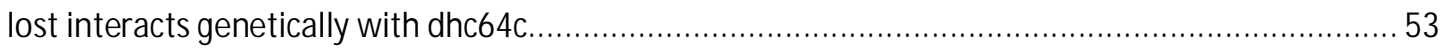

No detectable change in pgcRNA or peptide localization in maternal lost mutants......................... 54

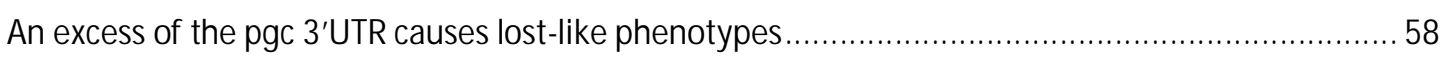

The amount of oskRNA in the PGCs is increased in maternal lost mutants ...................................6 60

The localizion of osk RNA and protein is highly dynamic during PGC formation................................ 62

M aternal expression of osk-nos-3'UTRis sufficient to cause lost-like phenotypes........................... 68 


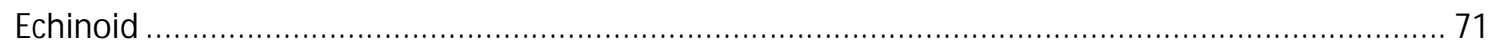

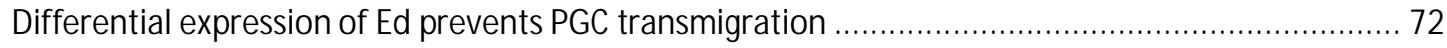

M echanism underling the inhibition of PGC transmigration ......................................................... 73

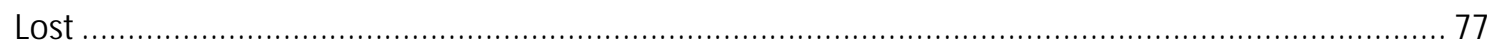

Posterior and PGC development is affected in maternal lost mutants ......................................... 77

Lost affects the localization of osk RNA

Osk protein and oskRNA are part of different RNP particles ........................................................ 81

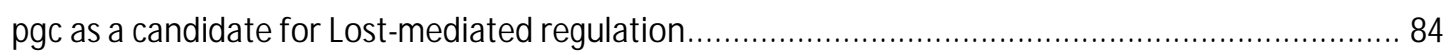

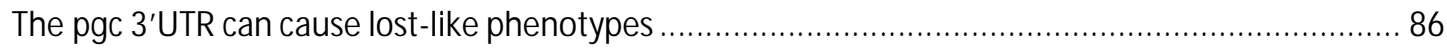

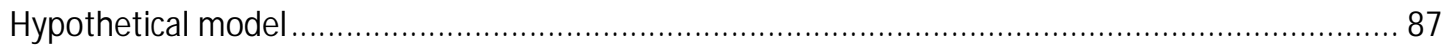

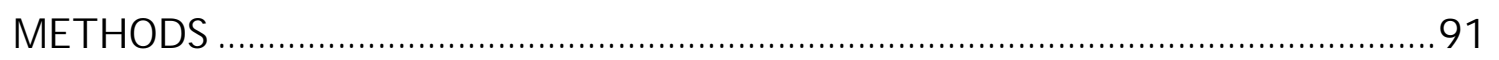

Fly methods

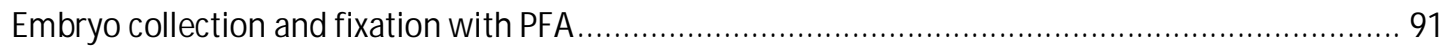

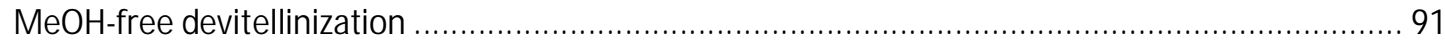

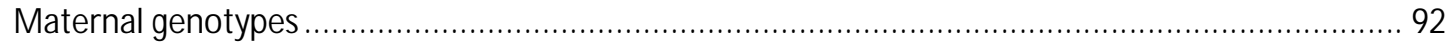

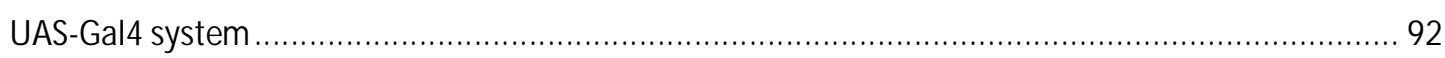

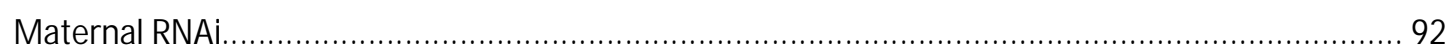

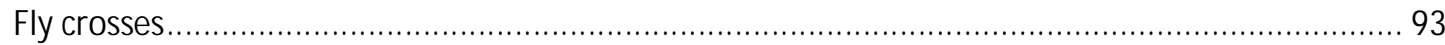

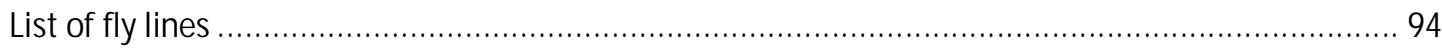

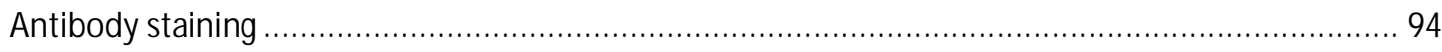

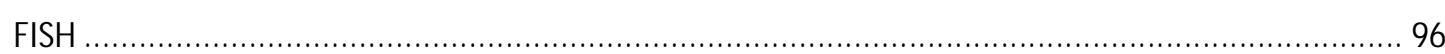

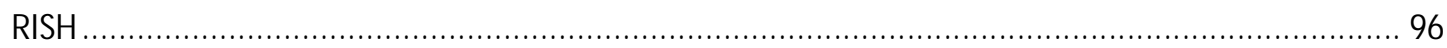

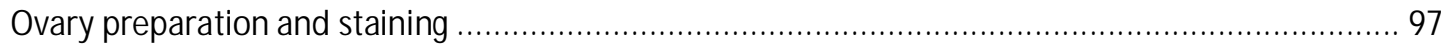

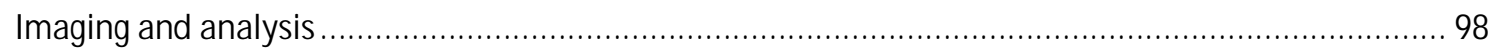

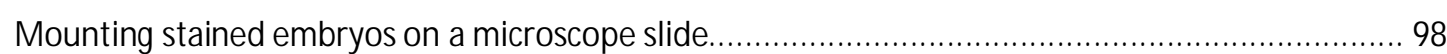

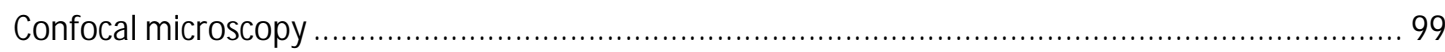

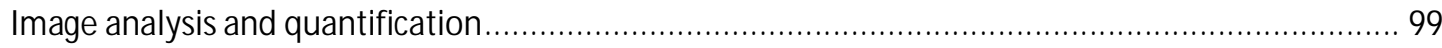

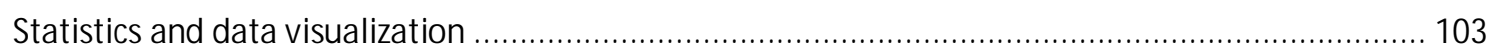

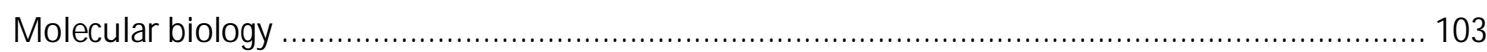

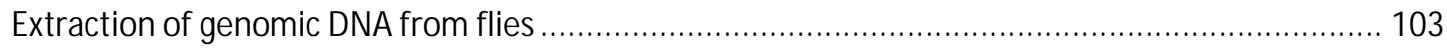

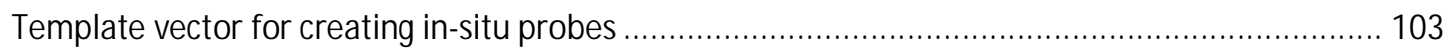




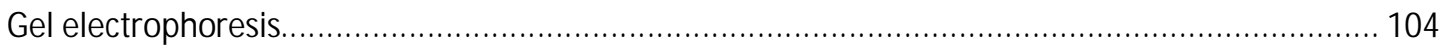

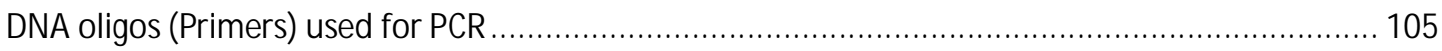

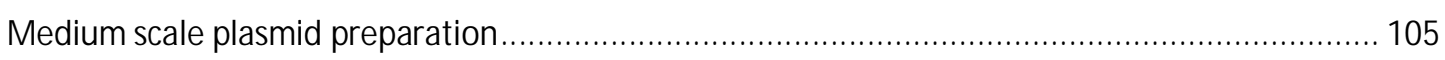

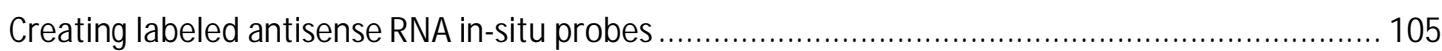

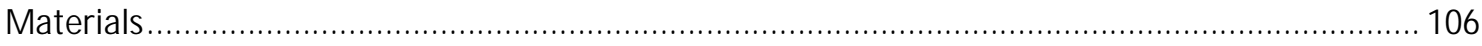

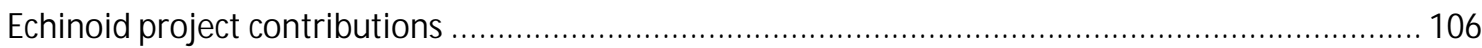

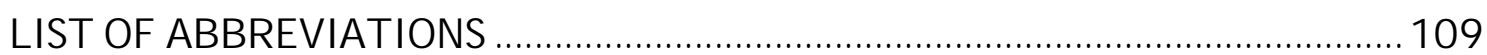

LST OF GENES........................................................................................................................ 111

LST OF FIGURES.................................................................................................................................. 113

DEALARATION............................................................................................................................................. 115

REFERENCES............................................................................................................................ 117

OURRIOLUMVTAE..........................................................................................................1 131

APPENDIXI: PROTOOOLS..................................................................................................... 133

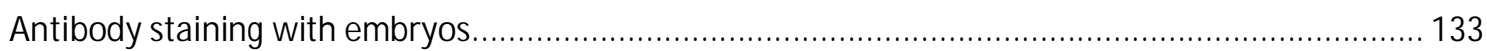

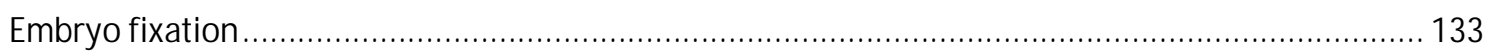

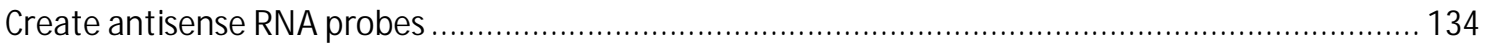

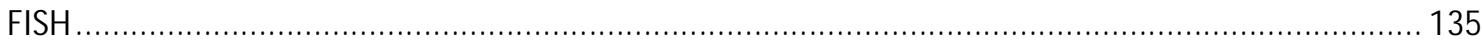

APPENDIXII: FII MACROS......................................................................................................... 137

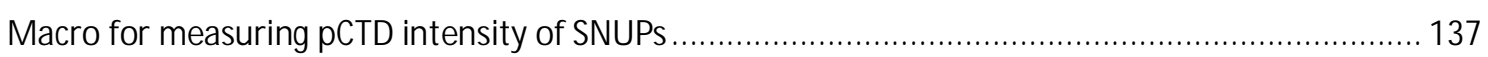

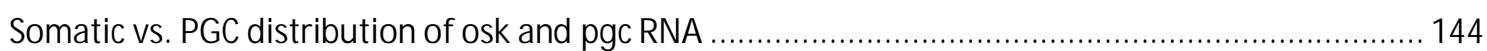

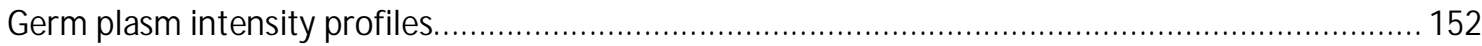

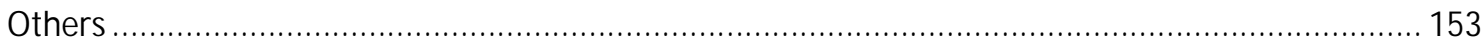





\section{Summary}

The germline cells are of fundamental importance for all animals as they have the ability to give rise to the next generation. Their precursors, the primordial germ cells (PGCs), are determined very early during animal development. To form the embryonic gonad they must come together with the somatic gonadal precursor cells (SGPs) which are often specified at a different location within the embryo. This requires the PGCs to actively migrate towards the SGPs.

In Drosophila, the PGCs arise from a specialized cytoplasm, the germplasm, which is deposited at the posterior pole of the oocyte during oogenesis. They form earlier than the somatic epithelium and are kept outside the embryo until gastrulation. Later they start their active migration towards the SGPs in the lateral mesoderm. If they fail to reach their destination they are removed by programmed cell death.

In this thesis two gene functions have been investigated which are required for the early separation of the PGCs from the somatic epithelium. If these gene functions are absent the PGCs migrate through the epithelium prematurely. Both genes have overlapping mutant phenotypes but function using completely different mechanisms.

echinoid (ed, CG12676) codes for a homophilic cell adhesion molecule which is expressed in the somatic epithelial cells but not in the PGCs during early embryogenesis. If Ed is removed from the soma the PGCs start to traverse the underlying epithelium already shortly after cellularization. Since this phenotype can also be observed when ed is ectopically expressed within the PGCs in addition to its expression in the epithelial cells the untimed transmigration is not due to Ed functioning as a normal cell adhesion factor. This rather demonstrates that the interface between $e d$ expressing and $e d$ nonexpressing cells is crucial to avoid untimed migration of the PGCs. These interfaces and their biological relevance have been studied before in other contexts like dorsal closure. It was shown that the differential expression of $e d$ is sufficient to trigger the formation of an actomyosin cable within the ed expressing cells at the interface. Here, the existence of a similar mechanism could also be demonstrated: In wild type embryos an apical meshwork of actomyosin is present in the epithelial cells beneath the PGCs. This 
actomyosin network is weakened or disrupted if Ed is removed from the epithelium or ectopically expressed within the PGCs. This result strongly supports a model in which actomyosin-mediated tension within the epithelial sheet is essential to inhibit the transmigration of PGCs. Such a regulatory network would represent the first example a system in which the tension within 2D-epithelia inhibits the invasion of overlying cells. However, the molecular mechanisms by which the differential expression of Ed triggers the formation of the apical actomyosin meshwork and its interaction with the well characterized E-cadherin actin meshwork at the adherence junctions remain to be investigated.

lost (CG14648) codes for highly conserved putative RNA binding protein. It is has been found in ribonucleoprotein particles (RNPs) like the splicosome or sponge bodies. Furthermore, together with Rump (a homolog of hnRNP that binds nos RNA) it has a function in the accumulation of the germ plasm during late oogenesis. lost RNA and protein are maternally provided and ubiquitously present in the early embryo. In embryos which lack the maternal lost contribution PGCs are migrating through the epithelium prematurely. This is likely to be caused by a reduction of transcriptional activity found only in the somatic nuclei beneath the PGCs resulting in cellularization defects at the posterior pole. Furthermore, the PGCs, which are transcriptionally silent in wild type embryos, show ectopic transcriptional activity in maternal lost mutant embryos.

All observed lost mutant phenotypes are specific for the posterior pole or the PGCs although Lost is ubiquitously present in the early embryo. Since Lost is a putative RNA binding protein and has been found in RNPs the localization pattern of several germ plasm RNAs was analyzed in maternal lost mutant embryos. It was confirmed that osk RNA, unlike other germ plasm RNAs, is not actively transported into the PGCs but largely remains in the soma in wild type embryos. In maternal lost mutants, however, the amount of osk RNA within the PGCs is increased fourfold. This mislocalization is sufficient to cause ectopic transcriptional activity within the PGCs and an increased rate of PGC transmigration as embryos which have osk RNA ectopically loaded into the PGCs by fusing the nos 3'UTR to the osk ORF show the same phenotypes. The amount or localization of Osk protein, however, does not differ from wild type neither in 
maternal lost mutants nor in embryos having osk RNA ectopically brought into the PGCs. So an increased transport of osk RNA into PGCs or its reduced degradation in the PGCs is sufficient to cause posterior cellularization defects with transmigrating PGCs and an increased number of transcriptionally active PGCs.

A candidate downstream effector could be $p g c$, another germ plasm component, which codes for a peptide responsible for the transcriptional silencing of the PGCs. pgc RNA partially inhabits the same RNP as osk RNA in early embryos.

The lost mutant phenotype combines features from pgc LOF (active transcription in PGCs) and pgc GOF (less transcription in posterior somatic nuclei, cellularization defects and transmigrating PGCs). This could be explained by an impaired $p g c$ RNA transport into the PGCs leading to more $p g c$ in the soma and less in the PGCs; yet quantitative analyzes did not detect a change in $p g c$ RNA localization nor a clear temporal or spatial difference in translation in maternal lost mutants after PGC formation. However, the average relative $p g c$ FISH staining intensity in the center of the germ plasm before PGC formation is increased in maternal lost mutants compared to wild type embryos. This could reflect a change in the $p g c$ RNP structure which affects the accessibility of $p g c$ RNA for the FISH probe.

Furthermore, the results show that the expression of a transgene consisting of the GFP ORF and the pgc 3'UTR is sufficient to cause lost-like phenotypes. Taken together, the loss of lost activity, an increased amount of osk RNA within the PGCs and maternal overexpression of the $p g c$ 3'UTR all lead to the same phenotypes: Ectopic transcriptional activity of PGCs and premature transmigration of PGCs through the underlying epithelium presumably caused by a reduced transcriptional activity of the posterior somatic cells.

Although the mechanisms regulating the gene activity remain unknown, it is tempting to speculate that Lost protein is a part of the osk-pgc RNP affecting osk RNA localization or its stabilization in the PGCs and by that indirectly regulating the activity of Pgc protein. However, more experiments are needed to identify the mechanisms and factors which govern the complex temporal and spatial regulation of the germline determinants. 



\section{Introduction}

The development of higher animals is an intriguing process as the whole body with its high number of different tissues originates from a single cell, the fertilized egg or zygote. Early in development this cell divides rapidly giving rise to a high number of cells which successively become more specialized and eventually form differentiated tissues and organs. The generation of different developmental fates depends on external cues like signaling molecules/morphogens or can be generated by asymmetrical cell divisions resulting in the uneven distribution of cytoplasmic determinants. Once different cell fates are established, it is essential to prevent these cell populations from mixing, which is usually achieved through differential expression of cell adhesion molecules. So both the establishment and the maintenance of different cell populations are of fundamental importance for all multicellular animals.

The germline cells are the only cells which have the ability to form eggs or sperm and thus give rise to the next generation. This makes them fundamentally different from the somatic cells. The germ line is specified early during development as primordial germ cells (PGCs). In contrast to the somatic cells, which differentiate into all kinds of cell types and mostly stop dividing, the germline cells must not differentiate to keep their pluripotency and their ability to divide. The establishment of the germline cells and their separation from the differentiating somatic surroundings is therefore crucial for all animals.

\section{Germ plasm biogenesis - oogenesis}

The ovaries of Drosophila are the largest organ in the adult females. They consist of about 18 ovarioles, each of which can be seen as an "assembly line" for the production of oocytes (Fig. 1 A) (Bastock and Johnston 2008). Its anterior tip, the germarium, harbors the germline stem cells (GSCs) as well as the somatic follicle stem cells (Bate 
and Martinez-Arias 1993). The GSCs divide asymmetrically giving rise to another GSC and a daughter cell which starts to differentiate (Spradling et al. 1997). The daughter cell divides four times with incomplete cytokinesis which results in a 16-cell cyst. The cyst cells are connected to each other via cytoplasmic bridges termed ring canals. One of the 16 cells will become the oocyte; the other ones will develop into polyploid nurse cells (Roth and Lynch 2009). This 16-cell cyst is then ensheathed by somatic follicle cells to form the egg chamber with the developing oocyte located at its posterior side (Roth and Lynch 2009). The nurse cells produce large amounts of proteins, RNAs, ribosomes and mitochondria which are transported along the microtubules through the ring canals into the oocyte. Later the nurse cells empty their content into the oocyte in a process called nurse cell dumping and undergo programmed cell death (Wheatley et al. 1995, Nezis et al. 2000).

The mature oocyte contains localized RNAs which define the body axis of the future embryo. At the anterior pole of the oocyte bicoid RNA is anchored (St Johnston et al. 1989) while the posterior pole holds the germ plasm where the polar granules are positioned (Mahowald 1962). These are large ribonucleoprotein particles (RNPs) which contain abdominal patterning information as well as the germ line determinants (Thomson et al. 2008). The assembly of the germ plasm, which is directed by Oskar (Osk), has been extensively studied during oogenesis (Mahowald 2001, Lehmann 2016). During stages 1-6 of oogenesis osk RNA is transcribed in the nurse cells and then transported into the oocyte via Dynein along microtubules (Fig. $1 \mathrm{~B}$ ) (Ephrussi et al. 1991, Jambor et al. 2014). At stage 7 a signal from the posterior follicle cells triggers a reorganization of the microtubules within the oocyte, which now nucleate from the cortex (Roth et al. 1995, Shulman et al. 2000). Their +-ends point in all directions with a slight bias towards posterior (Khuc Trong et al. 2015). osk RNA, which is now transported along the microtubules via Kinesin, accumulates at the posterior pole (Fig. 1 C) (Zimyanin et al. 2008). Recently it was shown that Actin related protein 1 (Arp1), which is a subunit of the dynactin transport complex, plays a role in promoting the growth of the microtubules into the posterior cortex by preventing their collapse (Nieuwburg et al. 2017). Dynactin is transported along the microtubules via Kinesin-1 to the posterior pole where it accumulates and stabilizes the microtubules which allows 
A

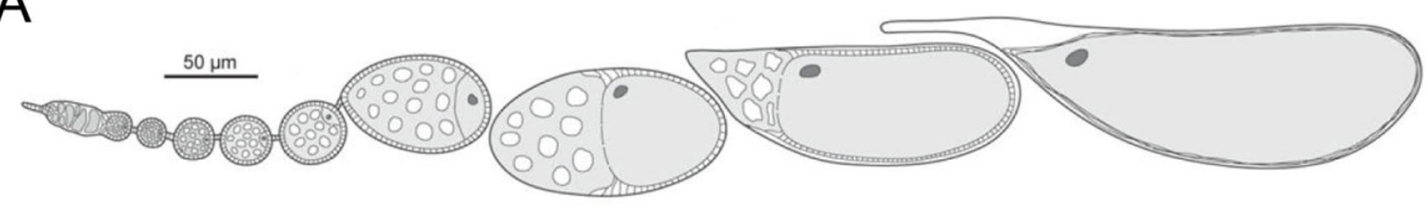

B
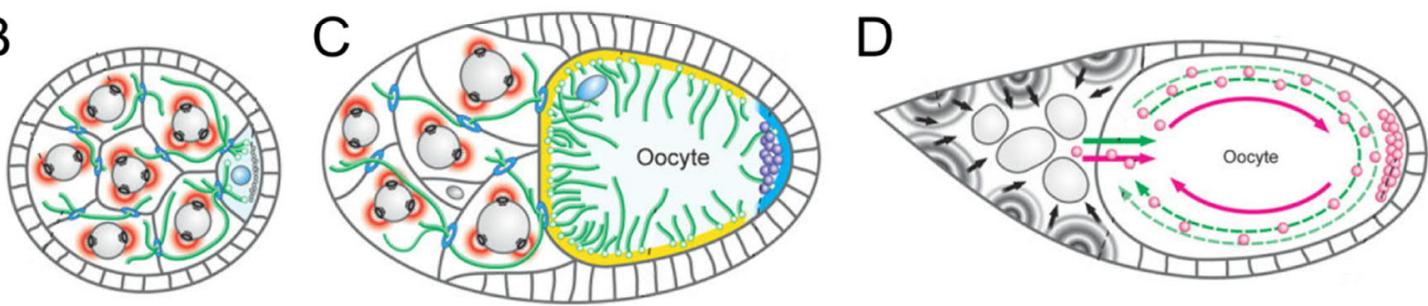

Figure 1: The germ plasm is deposited at the posterior pole during oogenesis. (A) Schematic representation of an ovariole with different stages of oogenesis. The anterior tip, the germarium (left), contains the stem cells. The right image is the mature egg. (B) Stage 5 egg chamber showing the microtubule network (green) connecting the nurse cells with the oocyte. The oocyte is at the posterior pole of the egg chamber (right). (C) After initial polarization the microtubules (green) in the oocyte nucleate from the anterior and lateral cortex (yellow) and extend inwards with a slight bias towards posterior. This leads to the accumulation of osk RNA (blue) at the posterior pole. (D) Nurse cell dumping and ooplasmic streaming. The nurse cells (left) empty their content into the oocyte and undergo programmed cell death. Ooplasmic streaming ensures the content reaches the posterior pole. nos RNA (pink) and other germ plasm components are trapped at the posterior pole and continue to accumulate. (adapted from Lehmann (2016))

Kinesin-1 to transport more Dynactin. This positive feedback loop ensures the microtubules reach the posterior cortex. If this function of Arp1 is prohibited by a point mutation osk RNA is not properly transported and the amount of osk RNA which is anchored at the posterior cortex is severely reduced (Nieuwburg et al. 2017).

There are two isoforms of Osk, both of which are only translated at the posterior cortex (Markussen et al. 1995, Vanzo et al. 2007). The long isoform of Osk promotes the anchoring of the germ plasm creating a positive feedback loop (Tanaka et al. 2011). The short isoform of Osk then acts as a master regulator of posterior and germ plasm development (Lehmann 2016) by recruiting Vasa and Tudor (Breitwieser et al. 1996, Anne 2010). Tudor acts as a scaffold for germ plasm assembly and recruits Aubergine (Arkov et al. 2006, Liu et al. 2010). Germ plasm RNAs like nos, gcl and pgc are then trapped and anchored to the posterior pole (Forrest and Gavis 2003, Sinsimer et al. 2013) where they form large, posterior localized RNPs, which contain the germ line determinants, termed germ granules. At stage 10 of oogenesis the microtubules align in 
the cortex of the oocyte (Dahlgaard et al. 2007). The nurse cells undergo programmed cell death and dump their content into the oocyte (Wheatley et al. 1995, Nezis et al. 2000). Ooplasmic streaming, driven by Kinesin, mixes the ooplasm with the contents of the nurse cells (Fig. 1 D) (Serbus et al. 2005). During this phase osk RNA as well as other germ plasm components continue to accumulate at the posterior pole of the oocyte (Snee et al. 2007). Lost, in a complex with Rump, has been shown to play a role in this process (Sinsimer et al. 2011).

The mechanisms which restrict the translation of osk RNA to the posterior pole of the developing oocyte have been extensively studied (see Lehmann (2016) for a review). During its transport from the nurse cells into the oocyte osk RNA is, among others, bound to Staufen (Stau) and Bruno (Bru) (St Johnston et al. 1991, Kim-Ha et al. 1995). Stau has been shown to be important for the transport of osk RNA along microtubules as well as for its translation when it reaches the posterior pole (Micklem et al. 2000). Bru represses osk RNA translation during the transport in a complex with Cup, a 5'UTR cap binding protein (Nakamura et al. 2004). It has been suggested that the concentration of Bru relative to local osk RNA levels is crucial for repressing the translation. During transport, when osk RNA levels are low, Bru binds to osk RNA and blocks translation. In contrast, at the posterior pole of the oocyte, where osk RNA molecules accumulate, Bru levels are not high enough to suppress translation of osk resulting in the localized production of Osk protein (Kanke and Macdonald 2015). It has been shown that the poly-A binding protein Orb and Poly-A-Binding Protein (PABP) are required for $o s k$ translation indicating that polyadenylation is an essential step (Castagnetti and Ephrussi 2003, Vazquez-Pianzola et al. 2011).

Besides coding for Osk protein it has been suggested that osk RNA has additional functions during oogenesis. In ovaries from osk RNA null mutant mothers oogenesis halts during early stages, before Osk protein is normally produced (Jenny et al. 2006). Since this phenotype can be rescued by reducing the levels of Bru protein (Kanke et al. 2015) it has been suggested that in wild type ovaries osk RNA is required for reducing the levels of unbound Bru which would otherwise interfere with the translation of genes required for early oogenesis. 


\section{Early embryogenesis}

Drosophila embryogenesis starts with a rapid series of synchronous mitotic divisions without cytokinesis. During the ninth division cycle the majority of the nuclei migrate to the periphery of the embryo where they form a monolayer in the cortex (Rabinowitz 1941). The nuclei that reach the posterior pole, where the germ plasm resides, will bud out and form the primordial germ cells (PGCs) or pole cells (Rabinowitz 1941). The somatic nuclei continue to divide synchronously while the PGCs divide asynchronously and at a lower rate (Rabinowitz 1941).

After a total of 14 cycles of mitotic divisions the somatic nuclei stop dividing and cellularization starts (Fig. 2 A). During this process the plasma membrane grows in between the somatic nuclei, encloses them and simultaneously forms the about 6000 cells of the embryonic epithelium (Mazumdar and Mazumdar 2002). After the last mitotic division the centrosomes locate apically of the nuclei with the microtubules extending their minus-ends basally towards the interior of the embryo forming a basketlike structure around the nuclei (Warn and Warn 1986). slow-as-molasses (slam) RNA and protein accumulate in the cortex between the nuclei (Lecuit et al. 2002). slam is maternally contributed as well as zygotically transcribed (Acharya et al. 2013). The localization of slam RNA and protein depend on each other. Slam protein then recruits DRhoGEF2 which in turn locally activates the small GTPase Rho1 (Wenzl et al. 2010). This leads to the local recruitment and stabilization of actomyosin at the site of membrane invagination (Padash Barmchi et al. 2005). The invagination front, termed furrow canal (FC), moves inwards separating the nuclei (Fig. 2 B). slam RNA and protein stay associated with the FC during the invagination (Acharya et al. 2013). During cellularization the apical-basal polarity of the forming epithelium is established which involves several highly conserved protein complexes (Laprise and Tepass 2011). The Drosophila PAR-3 homolog Bazooka (Baz) is a key player in establishing the apical domain of a cell (Tepass 2012). Very early during cellularization Baz is recruited to the apicolateral plasma membrane via microtubules and the apical actin cytoskeleton (Fig. 2 B) (Harris and Peifer 2005). There it serves as a platform for the recruitment of other apical domain proteins like atypical Protein Kinase C (aPKC), Crumbs $(\mathrm{Crb})$ and 


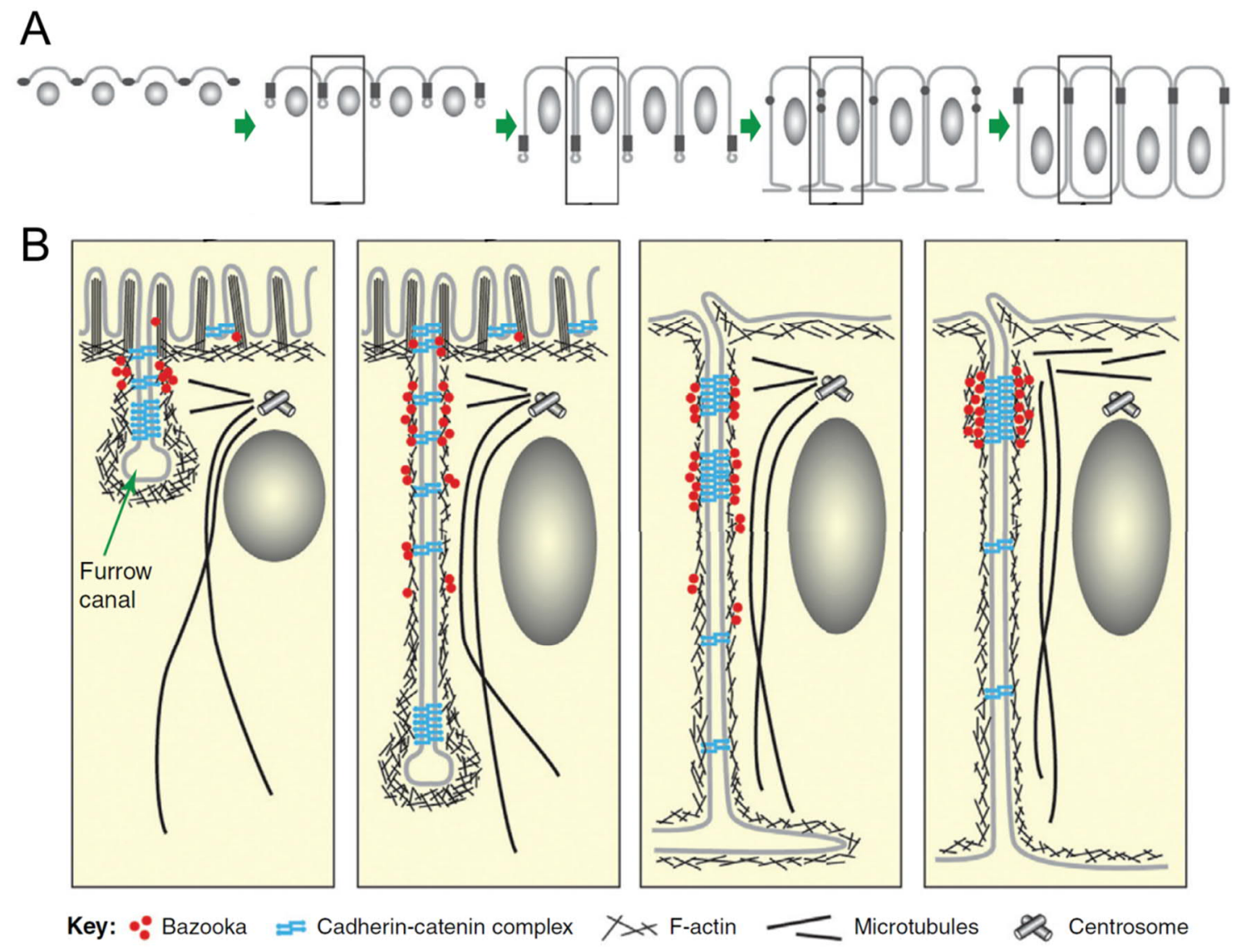

Figure 1: Cell polarity and adhesion are established during cellularization. (A) Schematic representation of cellularization. The somatic nuclei (grey) sit beneath the plasma membrane. Then the furrow canal (FC) forms, the membrane invaginates in between the nuclei. After passing the nuclei the contractile actomyosin at the furrow canal constricts pinching off individual cells. (B) Establishing cell adhesion. Bazooka (Baz) defines the subapical membrane domain. Just apical of the FC an adherens junction (AJ) forms, which stays basal at the FC. While the invagination proceeds spot adherens junctions (SAJ) form in the lateral membrane, which then condense in the subapical domain, while the basal AJ is disassembled. When cellularization is complete apical adherens junctions (AAJ) have formed. (adapted from Laprise and Tepass (2011))

Stardust (Std) (Tepass 1996, Harris and Peifer 2004, Harris and Peifer 2005). A complex of Discs Large (Dlg), Scribbled (Scrib) and Lethal Giant Larvae (Lgl), which is recruited to the lateral membrane domain, prevents the apical domain from expanding basally (Bilder et al. 2000, Bilder and Perrimon 2000).

Cell adhesion is also established during cellularization with two distinct types of adherens junctions (AJ) forming: The transient basal adherens junctions form close to the FC and follow the invagination (Fig. 2 B) (Mazumdar and Mazumdar 2002). Spot 
adherens junctions form in the lateral membrane and then get recruited to the subapical domain by Baz (McGill et al. 2009). There they start clustering together and slowly form a mature zonula adherens during gastrulation. AJ contain DE-Cadherin (Shotgun, Shg), a highly conserved homophilic cell adhesion molecule, $\beta$-Catenin (Armadillo, Arm) and $\alpha$-Catenin, which link the intracellular domain of Shg to the actin cytoskeleton (Tepass et al. 2001). Once the FC passes the nuclei the invagination accelerates and eventually the actomyosin in the FC constricts and pinches off individual cells (Fig. 2 B) (Mazumdar and Mazumdar 2002).

When cellularization is complete gastrulation starts (reviewed by Leptin (1995)). At the ventral side of the embryo the mesoderm starts to invaginate. The germ band extension (GBE) shifts the posterior midgut (PMG) anlage dorsal and anterior. The PGCs form a loose aggregation on top of the PMG epithelium and are passively carried along (StarzGaiano and Lehmann 2001). During this process the length of the germ band increases dramatically which is driven by cell intercalation and oriented cell division (Irvine and Wieschaus 1994, da Silva and Vincent 2007). The PMG epithelium then invaginates forming the posterior gut pocket while the germ band continues to extend. Like the mesoderm invagination the PMG invagination is initiated by the apical constriction of the respective epithelial cells (Leptin 1995). The apical constriction is driven by contractile actomyosin located in the apical and subapical cortex of the cells. All these morphogenetic tissue movements must take place without compromising epithelial integrity. This requires extensive and coordinated rearrangement of AJs and their link to the cortical actin cytoskeleton (Rauzi et al. 2015)

\section{PGC development}

The PGCs are the first individual cells that form during embryogenesis (Fig. 3 A). During their formation the germ plasm is actively transported into the budding PGCs along astral microtubules (Lerit and Gavis 2011). As soon as a PGC is formed it ceases actively transcribing genes (Seydoux and Dunn 1997) while the somatic nuclei stay 
transcriptionally active. This global transcriptional silencing in the PGCs is mediated by Polar granule component (Pgc), a 71 amino acid peptide. Pgc binds to positive Transcription Elongation Factor b (pTEFb) thereby preventing it from phosphorylating the carboxyterminal domain (CTD) of RNA polymerase II which is required for active transcription (Hanyu-Nakamura et al. 2008). pgc RNA is part of the maternally contributed germ plasm and is only translated after the PGC formation to ensure that transcription is only shut down in the PGCs and not in the somatic nuclei (Rangan et al. 2009).

At the beginning of gastrulation the PGCs are passively shifted towards dorsal and anterior with the elongating germ band (Fig. 3 B). Eventually they are internalized and reside inside the gut pocket (Starz-Gaiano and Lehmann 2001) forming a tight cluster (Kunwar et al. 2008). At embryonic stage 9, when the germ band is fully extended, the PGCs lose their contact to each other and start to migrate out of the gut pocket as individual cells (Fig. 3 C) (Jaglarz and Howard 1995). At the same time the PGCs become transcriptionally active (Zalokar 1976). Two independent events have been shown to be essential for the transmigration. First, Trapped-in-endoderm-1 (Tre1), a Gprotein coupled receptor with unknown ligand, needs to be active in the PGCs. In maternal tre1 mutants the PGCs fail to disperse and stay clustered in the gut pocket (Kunwar et al. 2003, Kunwar et al. 2008). Second, the PMG epithelium needs to undergo an epithelial-to-mesenchymal transition (EMT) during which the PMG cells lose their apical-basal polarity and weaken their cell adhesion (Campbell et al. 2011). This allows the PGCs to migrate through the tissue (Seifert and Lehmann 2012). The EMT is independent of PGC development and required for the formation of the digestive tract (Reuter et al. 1993). The anterior midgut and the PMG cells both temporarily adopt mesenchymal behavior, migrate towards the center of the embryo and fuse to form the midgut (Reuter et al. 1993). In the PMG the EMT is triggered by the GATA factor Serpent (Srp), which represses the transcription of crb (Campbell et al. 2011). This leads to the loss of apical-basal polarity and to the delocalization of AJ proteins like Shg, which weakens the cell adhesion (Campbell et al. 2011). If the EMT in the PMG epithelium is blocked by mutating srp or huckebein $(h k b)$, the PGCs fail to leave the gut pocket (Jaglarz and Howard 1994, Campbell et al. 2011). Furthermore, 
A

$\underline{\text { Germ Cell Formation }}$

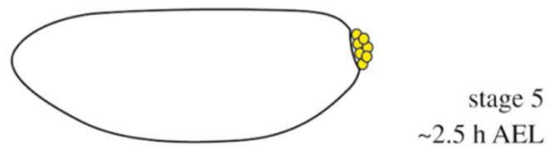

B

$\underline{\text { Germ Band Extension }}$

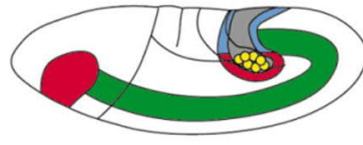

$\sim 3 \mathrm{~h} 45 \mathrm{~m}$ AEL

C Migration through Midgut

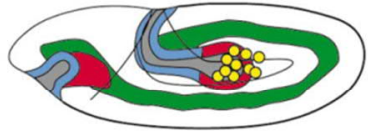

stage 9 $\sim 4.5$ h AEL

D Migration on Midgut and into Mesoderm

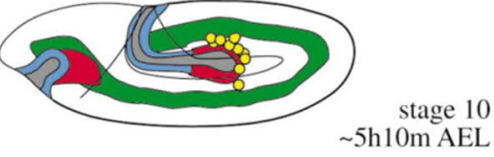

E Attachment to Lateral Mesoderm

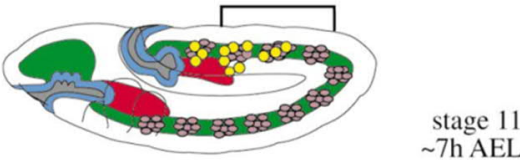

F Alignment with Gonadal Mesoderm

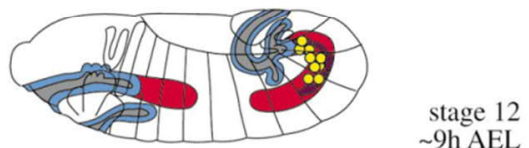

G

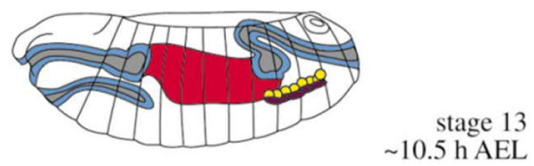

$\mathrm{H}$

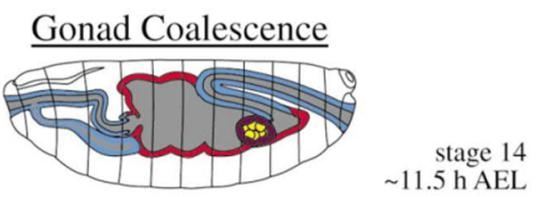

Seifert and Lehmann (2012) showed that weakening the cell adhesion in the PMG independent of the EMT triggered by srp by reducing the amount of $\mathrm{Crb}$ is sufficient for the PGCs to leave the gut pocket. This demonstrates that the weakening or disruption of epithelial integrity either by EMT or other means allows the PGCs to transmigrate.

Once the PGCs have left the gut pocket they migrate dorsally towards the trunk mesoderm where they split into two lateral groups (Fig. 3 D) (Sonnenblick 1941). Then they migrate along the lateral mesoderm towards posterior where they associate with the somatic gonadal precursor (SGP) cells and eventually form the embryonic gonad (Fig. 3 EH) (Starz-Gaiano and Lehmann 2001). The splitting and directed migration is regulated by to date unidentified repulsive and attractive cues.

Figure 2: Schematic representation of the migration of the PGCs. (A) The PGCs (yellow) are formed at the posterior pole. (B) During gastrulation the midgut (red) and the mesoderm primordia (green) are formed. The PGCs are carried passively on the posterior midgut (PMG) and get internalized in the gut pocket. (C,D) The PGCs actively migrate out of the gut pocket and towards the mesoderm. (E) The PGCs migrate into the lateral mesoderm and (F,G) align with the somatic gonadal precursor (SGP) cells. (H) During gonad coalescence the PGCs increase their cell adhesion, undergo compaction and are surrounded by the SGP. (adapted from Starz-Gaiano and Lehmann (2001)) 
However, several genes have been shown to play a role in producing or receiving the guidance cues (Santos and Lehmann 2004a).

Wunen (Wun) and Wunen-2 (Wun2) are transmembrane proteins with a lipid phosphate phosphatase enzymatic activity located in the extracellular domain of the protein (Sigal et al. 2005). wun2 is maternally provided to the PGCs while wun is mostly zygotically expressed in a pattern which provides guidance cues to the migrating PGCs. It is thought that Wun cleaves an unidentified, ubiquitously present phospholipid which acts as an attractant to the PGCs. The presence of Wun therefore reduces the concentration of that phospholipid which provides a negative guidance cue to the PGCs (Starz-Gaiano et al. 2001). wun is expressed in the ventral cells of the PMG epithelium at embryonic stages 9 and 10. when the PGCs leave the gut pocket. This negative cue directs PGCs migration towards dorsal into the trunk mesoderm. When in contact with the mesoderm the PGCs split into two lateral groups caused by wun expression in the central nervous system (CNS). This wun expression is believed to cause again a negative guidance cue along the midline by the degradation of the unknown phospholipid resulting in a gradient of this lipid which ensures an equal distribution of PGCs into two lateral clusters (Renault et al. 2010).

Besides providing guidance cues a phospholipid acts as a survival factor for the PGCs. It has been suggested the PGCs sense the unknown phospholipid via Wun2 which upon cleavage internalizes the lipid (Renault et al. 2004). wun2 RNA is maternally provided to the PGCs (Hanyu-Nakamura et al. 2004). The more wun2 RNA a particular PGC receives the higher is its chance for survival (Slaidina and Lehmann 2017). In embryos overexpressing wun in the mesoderm the PGCs die rapidly presumably because the phospholipid is cleaved by the ectopic Wun (Starz-Gaiano et al. 2001).

The migration of the PGCs towards the SGPs is regulated by columbus (clb), which codes for a 3-hydroxy-3-methylglutaryl-CoA reductase (HMGCoAR). clb is initially expressed throughout the mesoderm and later restricted to the SGPs, where it provides attractive guidance cues for the PGCs (Van Doren et al. 1998a). Overexpressing $c l b$ is sufficient to guide the PGCs into tissues they normally avoid (Van Doren et al. 1998a). 
HMGCoAR catalyzes the rate limiting step of the mevalonate pathway which synthesizes isoprenoids and sterols (Goldstein and Brown 1990). It has been shown that mutating different enzymes of the mevalonate pathway, including the $\beta$-subunit of the geranyl-geranyl transferase type I ( $\beta G G T I)$, results in similar PGC migration phenotypes, which points at a geranylized peptide which acts as a chemoattractant for the PGCs (Santos and Lehmann 2004b).

During germ band retraction the PGCs associate with the SGPs, which are located in the lateral mesoderm of parasegments 10 to 13 (Fig. 3 F,G) (Boyle and DiNardo 1995). The SGP cluster in parasegment 13 contributes to the gonad only in male embryos and is specified in a different way (DeFalco et al. 2003). During embryonic stage 14 the PGCs switch from a motile to a non-motile state and get surrounded by the SGPs (Fig. $3 \mathrm{H}$ ). This process, termed gonad coalescence, requires changes in cell adhesion between the PGCs as well as between the PGCs and somatic SGPs. The zinc transporter Fear of Intimacy (Foi) regulates the expression of Shg transcriptionally and posttranscriptionally, which is essential for this process (Jenkins et al. 2003, Van Doren et al. 2003, Mathews et al. 2006).

\section{Echinoid}

echinoid (ed) codes for a transmembrane homophilic cell adhesion molecule. The extracellular part of Ed contains 7 Immunoglobulin (Ig) superfamily domains and a fibronectin superfamily domain while the intracellular domain lacks any conserved protein domains (Vogel et al. 2003).

$e d$ RNA and protein are maternally provided to the embryo. It is expressed throughout embryogenesis in all epidermal tissues. ed RNA can be found in the embryonic epidermis, the developing trachea and in the midgut epithelium. 
Early studies focused on the role of Ed in modulating different signaling pathways. It was shown that Ed antagonizes the EGFR pathway during the development of the photoreceptors (Bai et al. 2001, Rawlins et al. 2003b). Ed was also shown to modulate Notch signaling during neurogenesis and thorax bristle patterning (Escudero et al. 2003, Rawlins et al. 2003a).

In maternal-zygotic ed mutant embryos the trachea show a convoluted phenotype with reduced intersegmental spacing. Tracheal fusion is also affected by an increased number of fusion events and atypical branching. Although being a cell adhesion molecule loss of Ed in the trachea has no effect on the function of septate junctions (Laplante et al. 2010).

More insight into the function of Ed in epithelial morphogenesis came from the analysis of ed mutant cell clones in the larval wing disc epithelium (Wei et al. 2005) and the follicular epithelium of the egg chamber (Laplante and Nilson 2006). Wei et al. (2005) could show that Ed is a part of the apical adherens junctions (AAJ) and can bind to Canoe (Cno), which interacts with the actin cytoskeleton. They also describe the border between $e d$ mutant cell clones and ed expressing surrounding tissue lacking any AJ components but instead being rich in actin filaments. Laplante and Nilson (2006) confirmed the findings of Wei et al. (2005) and focused more on the interface between $e d$-expressing and $e d$-non expressing cell populations. They describe an intercellular actomyosin cable forming at this interface. It was also shown that a similar actomyosin cable plays a role during the development of the dorsal appendages of the egg shell and during dorsal closure in mid-embryogenesis.

A more detailed analysis of the function of Ed during dorsal closure was later published by the same group (Laplante and Nilson 2011). It was shown that the differential expression of $e d$ (it is present in the lateral epidermis but not in the amnioserosa) creates an interface which defines the leading edge. An intercellular contractile actomyosin cable is formed at the interface which provides physical force that contributes to dorsal closure. If the interface between $e d$-expressing and $e d$-non expressing cells is eliminated 
(by either removing Ed from the epidermis or by ectopically expressing Ed in the amnioserosa) the actomyosin cable fails to form which leads to defects in dorsal closure.

During early embryogenesis the epidermal cells express ed while the PGCs at the posterior pole do not contain Ed. This creates another the interface between edexpressing and $e d$-non expressing cells. In this thesis, the functional relevance of this differential expression of $e d$ regarding the development of the PGCs is investigated.

\section{Lost}

The gene lost (CG14648) was first identified in a misexpression screen in muscle apodeme precursor cells where it caused aberrant muscle morphology and ectopic midline crossing of muscle fibers (Molitor 2002). This phenotype lead to further investigations of lost by Molitor (2002) and later Graf (2007).

The gene lost is located on the third chromosome at cytogenetic band 3R:82A6. It consists of 6 exons and codes for two isoforms, which result from using an alternative promotor (Molitor 2002). The mutant allele $l o s t^{2}$ was created by an imprecise jump-out of a P-element positioned in the first intron which resulted in a 1466 base pair (bp) deletion removing the entire second and a part of the third exon. The deletion also causes a frame shift which leads to an early termination (Molitor 2002).

lost mRNA can be found throughout Drosophila development, from the embryo to the adult fly (Molitor 2002). Besides the very early embryo (0-2h) and the adult female, which contain more lost RNA, the level of transcription is roughly constant (Gelbart and Emmert 2010).

Lost protein contains an N-terminal 5-formyltetrahydrofolate cyclo-ligase (5-FTHF cyclig) family domain and a C-terminal RNA recognition motif (RRM) superfamily domain, which belongs to the RNA recognition motif in vertebrate 
methenyltetrahydrofolate synthetase domain-containing (MTHFSD) protein subfamily (Marchler-Bauer et al. 2017). Although 5-FTHF cyclo-ligase enzymes play an important role in metabolism proteins containing a 5-FTHF cyc-lig domain and an RRM-MTHFSD domain are predicted to be RNA binding proteins (Marchler-Bauer et al. 2017). In fact, the human protein Methenyltetrahydrofolate synthetase domain containing (MTHFSD), which has a similar protein domain architecture as Drosophila Lost (Geer et al. 2002), has been shown to bind RNA in HeLa cells and in human embryonic kidney cells (Baltz et al. 2012, Castello et al. 2012).

Several sources point towards a function of Lost protein in RNA regulation or metabolism. An abstract describes a role of Lost for the localization of gurken mRNA during oogenesis (Lin et al. 2007), however, this result was never published in a peerreviewed publication. The same holds true for a personal communication in Dienstbier et al. (2009) which states that Lost binds RNA unspecifically. Furthermore, Lost protein has been found in the splicosome by mass spectroscopy analysis (Herold et al. 2009), where it physically interacts with Zn72D, a splicing factor for maleless (mle) (Worringer et al. 2009). Also Lost has been described as a sponge body component during oogenesis, where it can be co-immunoprecipitated with Trailer-hitch (Tral) if RNA is present (Snee and Macdonald 2009).

Sinsimer et al. (2011) published a role of Lost for the accumulation of germ plasm during oogenesis and nanos RNA (nos) localization in early embryos. They could show that in $50 \%$ of the embryos from lost mutant mothers nos RNA localization is slightly more diffuse than in wild type embryos (Sinsimer et al. 2011). Furthermore, they found that Lost interacts with the RNA binding protein Rumpelstiltskin (Rump) during oogenesis. Rump is a homolog of hnRNP and directly binds nos RNA (Jain and Gavis 2008). Using rump-lost double mutants, they showed that during late oogenesis the amount of germ plasm at the posterior pole of the oocyte is dramatically increased, a process which requires Lost and Rump. The initial assembly of the germ plasm during mid-oogenesis is not affected by the loss of Rump and Lost. Without the late phase of germ plasm accumulation the embryos show abdominal patterning defects and a reduced number of PGCs (Sinsimer et al. 2011). However, since all their results 
regarding the germ plasm only apply to rump-lost double mutants it is not clear which role Lost is playing in this process.

A genome-wide yeast-two-hybrid protein interaction screen identified six putative interaction partners for Lost (Giot et al. 2003). One of these putative interactors is Actin related protein 1 (Arp1), whose interaction with Lost was verified by coimmunoprecipitation (Graf 2007). Arp1 is part of the Dynactin transport complex, which among others also contains Dynein Heavy Chain (Dhc) (Allan 1996). Lost partially colocalizes with Dhc in early embryos (Graf 2007) which suggests that Lost binds to Dynactin in vivo.

Molitor (2002) and Graf (2007) also showed that the PGCs in embryos from lost mutant mothers migrate through the PMG epithelium prematurely. A detailed quantitative analysis of this premature transmigration phenotype revealed that the number of transmigrating PGCs is considerably increased during embryonic stages 6 and 7 (Hertel 2011). Furthermore, it was shown that besides physically interacting with Arp1 Lost also interacts genetically with Arp1 and Dhc: In embryos from mothers heterozygous for lost and arpl or $d h c$, respectively, the number of transmigrating PGCs is as high as in embryos from homozygous lost mutant mothers (Hertel 2011). This indicates a functional relevance for the interaction of Lost with the Dynactin complex during early embryogenesis.

In this thesis, the mutant phenotype of lost during early embryogenesis is analyzed in detail. The localization patterns of several germ plasm RNAs are visualized and quantified to gain insight into the mechanisms leading to the observed phenotypes. A possible function of Lost for indirectly regulating the translation of germ plasm RNAs via altering the properties of RNPs is discussed as a working model. 



\section{$\underline{\text { Results }}$}

\section{Echinoid}

The echinoid project was started as a lab rotation and bachelor thesis project by Ninett Wolfram under the practical supervision by the author of this dissertation. Details about the contributions can be found in the Methods section.

\section{$\underline{e d \text { is expressed in the soma, but not in the PGCs }}$}

In order to verify the published ed expression during embryogenesis RNA in-situ hybridization (RISH) and antibody staining using a specific antiserum against the Ed protein were performed. ed mRNA is maternally provided and present in the whole blastoderm embryo except for the germline (Fig. 4 A) (Kumar et al. 2011). Later it is enriched in the developing tracheal system, the segment boundaries and the epithelia of the digestive system (data not shown, (Kumar et al. 2011)). Figure 4 B shows the posterior pole of a stage 5 wild type embryo stained with an antibody against Ed and Dapi to label the nuclei. The Ed protein is apically enriched in the forming epithelium

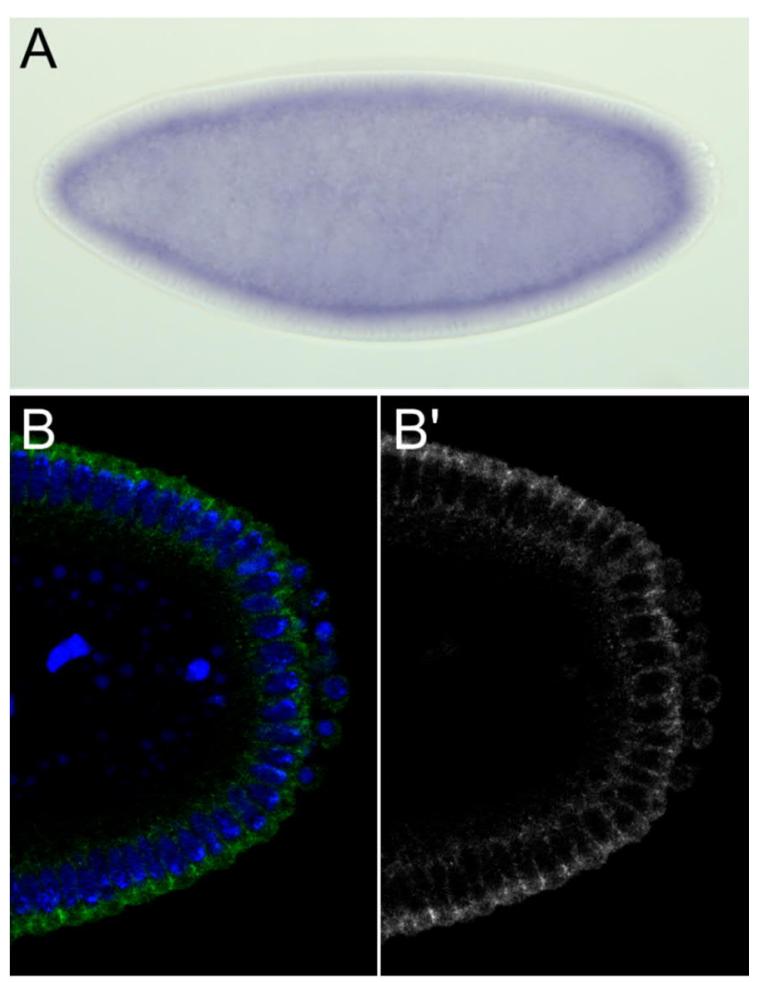

Figure 4: $\mathrm{Ed}$ is found the embryonic epithelium but not in the PGCs. (A) RNA insitu hybridization staining with antisense-ed of a wild type stage 5 embryo. Anterior is left, dorsal is up. ed RNA is present ubiquitously in the soma, but not in the PGCs at the posterior pole. (B) Confocal section of a stage 5 wild type embryo stained for Ed (green) and Dapi (blue). Ed protein $\left(\mathrm{B}^{\prime}\right)$ is located in the apicolateral membrane in the soma and is absent from the PGCs. 
during cellularization and absent from the PGCs (Fig. 4 B'). The apical localization shown here confirms published results that show that Ed can be detected in all epidermal tissues where it is co-localizing with apical adherence junction (AAJ) components like DE-cadherin (Wei et al. 2005).

Based on the absence of $e d$ mRNA and Ed protein from the PGCs an interface between ed expressing (somatic epithelium) and ed non-expressing (PGCs) cells is formed during cellularization. It has been suggested that such an interface could have a function in cell sorting where Ed activity is required to prevent the two cell populations from mixing (Lecuit 2005). However, such a function was only shown for cell populations of the same type, like ed-expressing and non-expressing follicle cells and eye discs cells when ed mutant clones were generated (Wei et al. 2005, Laplante and Nilson 2006). This raises the question whether the boundary between two independent tissues (the $e d$ positive epithelium and the $e d$-negative PGCs) is also required for the formation of a barrier that inhibits the mixing of these two cell types.

\section{Differential expression of $e d$ prevents the PGCs from transmigrating}

To address this hypothesis the interface between $e d$-expressing and $e d$-non expressing tissues needs to be removed by either removing $e d$ expression from the epithelium or by ectopically expressing $e d$ within the PGCs. In order to achieve the former embryos from ed mutant mothers need to be analyzed because ed RNA is maternally provided to the egg. Since $e d$ is an essential gene it is thus not possible to get homozygous mutant adult flies the germline clone (GLC) technique (Chou et al. 1993) has to be used to generate embryos without maternal $e d$ mRNA contribution (hereafter referred to as $e d^{F 72}$ GLC embryos). An alternative way to obtain embryos devoid of maternal $e d$ transcripts is the use of RNAi expressed exclusively in the germline of mothers ((Staller et al. 2013) see methods for details). To evaluate whether the PGCs mix with the underlying epithelium fixed embryos have been stained with an antibody against Vasa to label the PGCs and with an antibody which detects tyrosine-phosphorylated proteins (PY20) to label the somatic epithelium. Figure 5 shows maximum intensity projections of 3-6 confocal sections of posterior poles of wild type embryos (Fig. 5 A,D,G), ed ${ }^{F 72}$ GLC embryos 

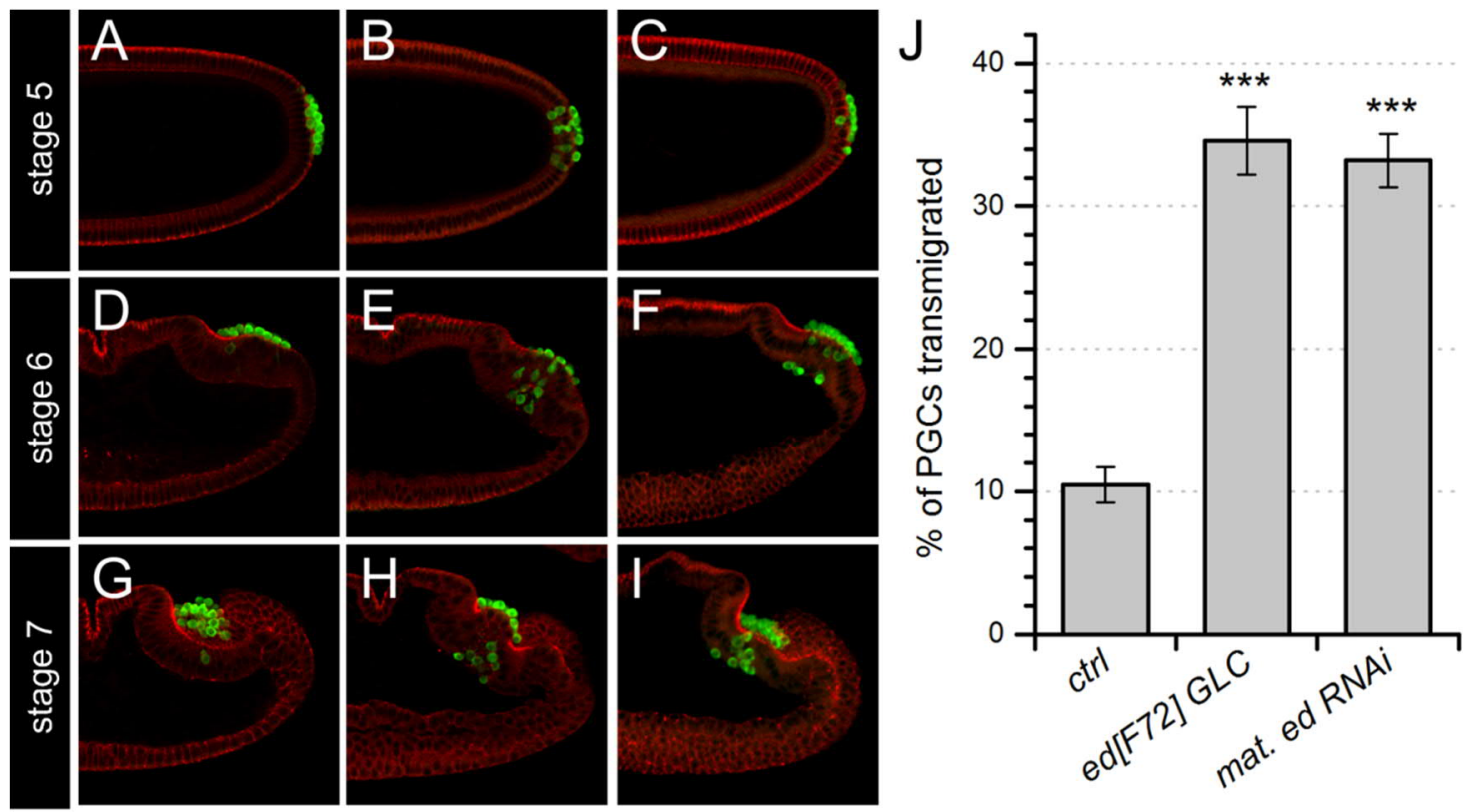

ctrl

$e^{F 72}$ GLC

mat. ed RNAi

Figure 5: In embryos which lack maternal Ed the PGCs mix with the underlying epithelium. (A-I) Maximum intensity projections of 2-5 confocal sections of posterior poles of embryos stained for Vasa (green) and phospho-tyrosine (red). (A,D,G) PGCs in wild type embryos reside at the posterior pole and are then passively moved into the forming PMG pocket. Only very few PGCs migrate through the epithelium. In embryos devoid of maternal Ed, $e d^{F 72} \mathrm{GLC}(\mathrm{B}, \mathrm{E}, \mathrm{H})$ and maternal ed RNAi (C,F,I) the PGCs migrate through the epithelium. (J) Quantification of the cell mixing phenotype. The columns show the average relative number of transmigrating PGCs during stages 6 and 7 of the three genotypes. Error bars show SEM. *** $\mathrm{p}<0.001$ Mann-Whitney-U test

(Fig. 5 B,E,H) and maternal ed RNAi embryos (Fig. 5 C,F,I) at stages 5, 6 and 7 of embryonic development.

In wild type embryos the PGCs remain at the site of their formation during cellularization (Fig. 5 A). With the onset of gastrulation they are shifted dorsally with the elongating germ band (Fig. $5 \mathrm{D}$ ) and then get internalized with the forming posterior midgut (PMG) pocket from stage 7 onward (Fig. 5 G). During these processes the PGCs remain outside the underlying somatic PMG epithelium. In $e d^{F 72}$ GLC embryos, however, the PGCs start to intermingle with the epithelium during the late phases of cellularization (Fig. 5 B). This becomes more apparent during stages 6 and 7 when more PGCs migrate in between the somatic epithelial cells (Fig. 5 E,H). The same phenotype is found in maternal ed RNAi embryos (Fig. 5 C,F,I). 
The relative number of PGCs which migrate in between the epithelium can be used measure the strength of the phenotype (Fig $5 \mathrm{~J}$ ). This reveals that in wild type embryos on average $10 \%$ of the PGCs transmigrate during stages 6 and 7. In $e d^{F 72}$ GLC or in maternal ed RNAi embryos $34 \%$ or $33 \%$ of the PGCs transmigrate, respectively. These changes are statistically significant when compared to wild type (Mann-Whitney-U test, $* * * \mathrm{p}<0.001)$.

This result shows that in embryos without a maternal ed mRNA contribution within the germline PGCs start to mix with the underlying epithelial cells. The effect is seen in $e d^{F 72}$ GLC embryos as well as in maternal ed RNAi embryos. However, the result obtained from ed mutants does not allow to distinguish if the observed cell mixing is caused by a cell autonomous function of Ed within the somatic epithelium or if it is caused by the loss of the interface between $e d$-expressing and $e d$ non-expressing cells.

To investigate if the loss of the interface between $e d$-expressing and $e d$ non-expressing cells is the cause of the observed cell mixing a second independent way of removing the interface is to ectopically express ed within the PGCs in addition to its normal expression in the somatic epithelium. In order to achieve this a transgene was constructed consisting of the ORF of ed fused to the 3'-UTR of the nanos (nos) gene. The 3'-UTR of nos targets the transgenic RNA to the germ plasm at the posterior pole of the embryo and into the PGCs (Van Doren et al. 1998b). The expression of the transgenic construct is controlled by the UAS-Gal4 system (Rorth 1998). Embryos from mothers which carry the transgene as well as a Gal4 driver which is active during oogenesis (hereafter referred to as ed-nos-3'-UTR embryos) will contain the transgenic fusion mRNA.

An ed RISH staining of ed-nos-3'-UTR embryos shows besides the endogenous maternal $e d$ mRNA a high concentration of $e d$ mRNA at the posterior pole before PGC formation (Fig. $6 \mathrm{~A}$ ) which is then transported into the PGCs (Fig. 6 B). Figure $6 \mathrm{C}$ shows a confocal section of the posterior pole of a stage 5 ed-nos-3'-UTR embryo stained with an antibody against Ed, an antibody against Vasa to label the PGCs and with Dapi to show the nuclei. Ed protein is detected apically in the somatic epithelium as well as within the PGCs (Fig. $6 \mathrm{C}^{\prime}$ ). This confirms that the transgenic ed RNA is indeed transported into the PGCs and translated and by that removing the interface 


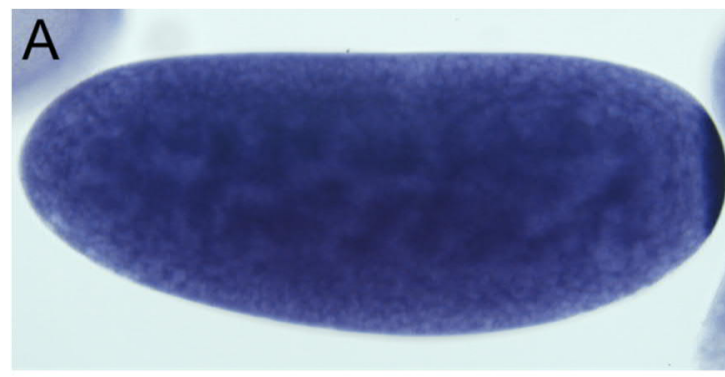

B
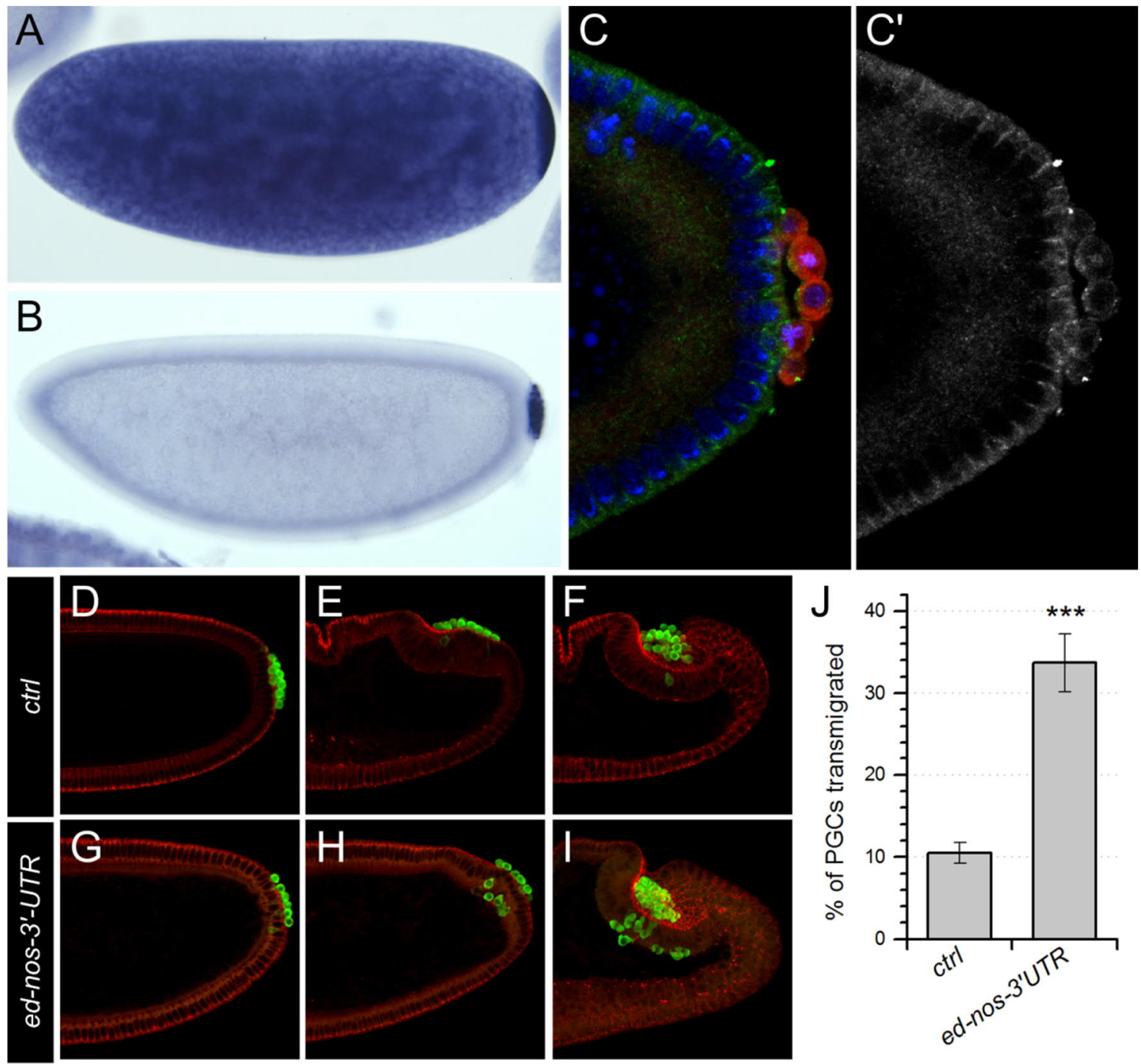

stage 5

stage 6

stage 7

Figure 6: Ectopic expression of ed within the PGCs leads to PGCs mixing with the epithelium. (A,B) RNA in-situ hybridization against $e d$ of $e d$-nos-3'UTR embryos. Anterior is left, dorsal is up. The probe detects the endogenous $e d$ mRNA present ubiquitously in the embryo and the transgenic ed RNA which (A) localizes to the germ plasm and (B) is later transported into the PGCs. (C) Confocal section of a stage 5 ed-nos-3'UTR embryo stained for Ed (green), Vasa (red) and Dapi (blue). ( $\left.C^{\prime}\right)$ Endogenous Ed protein is located in the apicolateral membrane in the soma. Ed protein from the transgene can be detected in the PGCs. (D-I) Maximum intensity projections of 2-5 confocal sections of posterior poles of embryos stained for Vasa (green) and phospho-tyrosine (red). (G-I) In ed-nos-3'UTR embryos the PGCs mix with the underlying epithelium during stages 6 and 7. (D-F) Wild type embryos are shown for comparison. (J) Quantification of the cell mixing phenotype. The columns show the average relative number of transmigrating PGCs during stages 6 and 7 of the wild type (ctrl) and ed-nos-3'-UTR embryos. Error bars show SEM. *** p $<0.001$ Mann-Whitney-U test.

between $e d$-expressing and $e d$ non-expressing cells without affecting the expression of $e d$ within the somatic cells. 
To evaluate whether the PGCs mix with the underlying epithelium fixed ed-nos-3'-UTR embryos are stained with an antibody against Vasa to label the PGCs and with the PY20 antibody to label the somatic epithelium. Figure $6 \mathrm{G}-\mathrm{H}$ shows maximum intensity projections of 3-6 confocal sections of posterior poles of ed-nos-3'-UTR embryos at stages 5, 6 and 7 of embryonic development. Wild type embryos of the same developmental stage are shown for comparison (Fig. 6 D-F). As in embryos devoid of maternal ed contribution (see Fig. 5) the PGCs in ed-nos-3'-UTR embryos mix with the underlying epithelial cells during stages 6 and 7 (Fig. 6 H,I). A quantification of the phenotype reveals that in ed-nos-3'-UTR embryos on average $34 \%$ of the PGCs migrate in between the epithelial cells (Fig. $6 \mathrm{~J}$ ). This differs significantly from wild type, where only $10 \%$ of the PGCs show that behavior (Mann-Whitney-U test, $* * * \mathrm{p}<0.001$ ).

This demonstrates that removing the interface between $e d$-expressing and $e d$ nonexpressing cells by ectopically expressing $e d$ within the PGCs leads to exactly the same phenotype than removing ed entirely from the early embryo: The PGCs mix with the underlying epithelial cells.

\section{Ed is removed from the PMG during transmigration at stage 10}

The analysis of ed mutants reveals an essential role for Ed protein in the PMG epithelium in early embryos (see Fig. 5) to prevent the migration of PGCs in between the PMG cells. At stage 9 of embryonic development, however, the PGCs start to actively traverse the midgut epithelium to migrate towards the lateral mesoderm (Jaglarz and Howard 1995). Therefore, either ed expression is lost in the PMG from stage 10 onwards or PGC start expression of $e d$ before they traverse the PMG.

To address this issue fixed wild type embryos have been stained with an antibody against Ed, an antibody against Vasa to label the PGCs and with Dapi to show the nuclei. Figure 7 A shows a mid-sagittal confocal section of a stage 9 embryo before the start of the active migration of the PGCs. Figure 7 B shows an optical cross section through the very same confocal stack at the position indicated by the dotted line in Figure 7 A. The dotted line in Figure 7 B indicates the position of the sagittal section 
shown in Figure 7 A. Figure $7 \mathrm{C}$ and

D show similar sections of a stage 10 embryo when most of the PGCs have left the gut pocket.

Before the active PGC migration Ed can be detected apically in the epithelial cells of the PMG (Fig. 7 A,B) which surround the cluster of PGCs. When the PGCs are migrating out of the gut pocket almost no $\mathrm{Ed}$ signal is present in the region of the epithelium where the PGCs are traversing, only a weak Ed signal is found in very few PMG cells (Fig. 7 C,D).

This shows that during stages 9 and 10 of embryonic development, when the PGCs actively migrate out of the gut pocket, Ed is locally removed from PMG epithelium allowing the PGCs to migrate in between the cells.
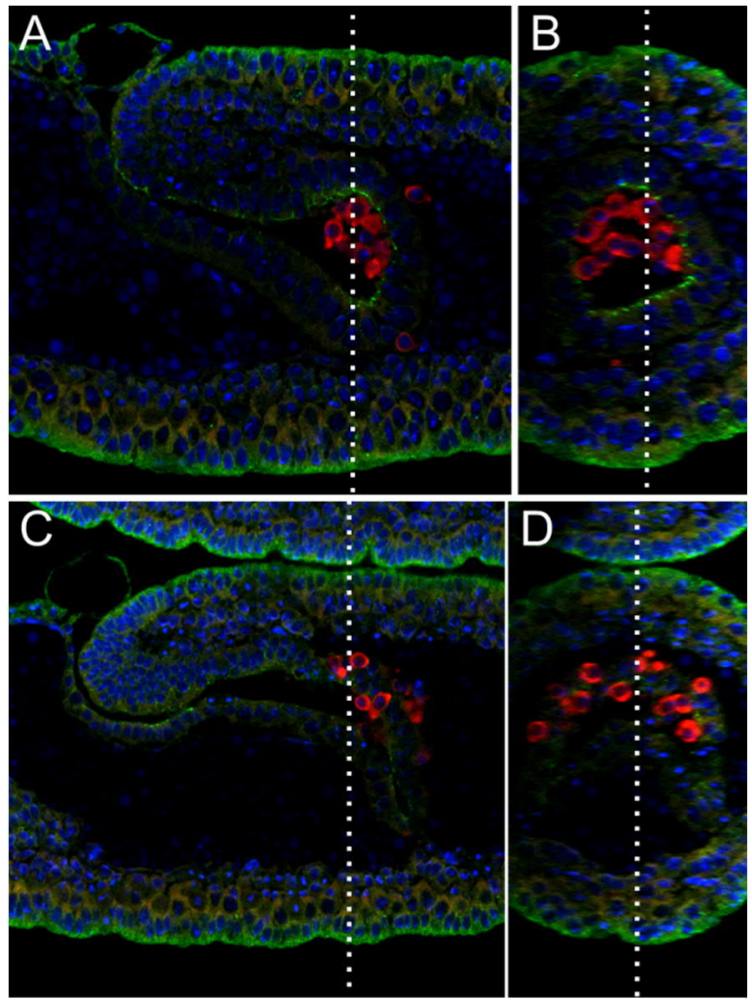

Figure 7: $\mathrm{Ed}$ is removed from the PMG cells when the PGCs transmigrate. (A-D) Wild type embryos stained for Ed (green), Vasa (red) and Dapi (blue). (A) Mid-sagittal and (B) cross section of a stage 9 embryo before the start of the active migration of the PGCs. The dotted lines indicate the position of the respective section. Ed (green) can be detected apically in the epithelial cells of the PMG. (C) Mid-sagittal and (D) cross section of a stage 10 embryo when the PGCs transmigrate. The dotted lines indicate the position of the respective section. Only a weak Ed signal is detected in a few PMG cells.

\section{Cellularization is not affected}

\section{by Ed}

Ed is part of the AAJ complex and indirectly linked to the actin cytoskeleton, but it is not essential for epithelial integrity (Wei et al. 2005). It could, however, have a function during cellularization, when the epithelium is established. To address the possibility fixed wild type, maternal $e d$ RNAi and $e d$-nos-3'-UTR embryos have been stained with antibodies against Discs-large (Dlg), Slam, Vasa and with Dapi. Dlg marks the lateral 

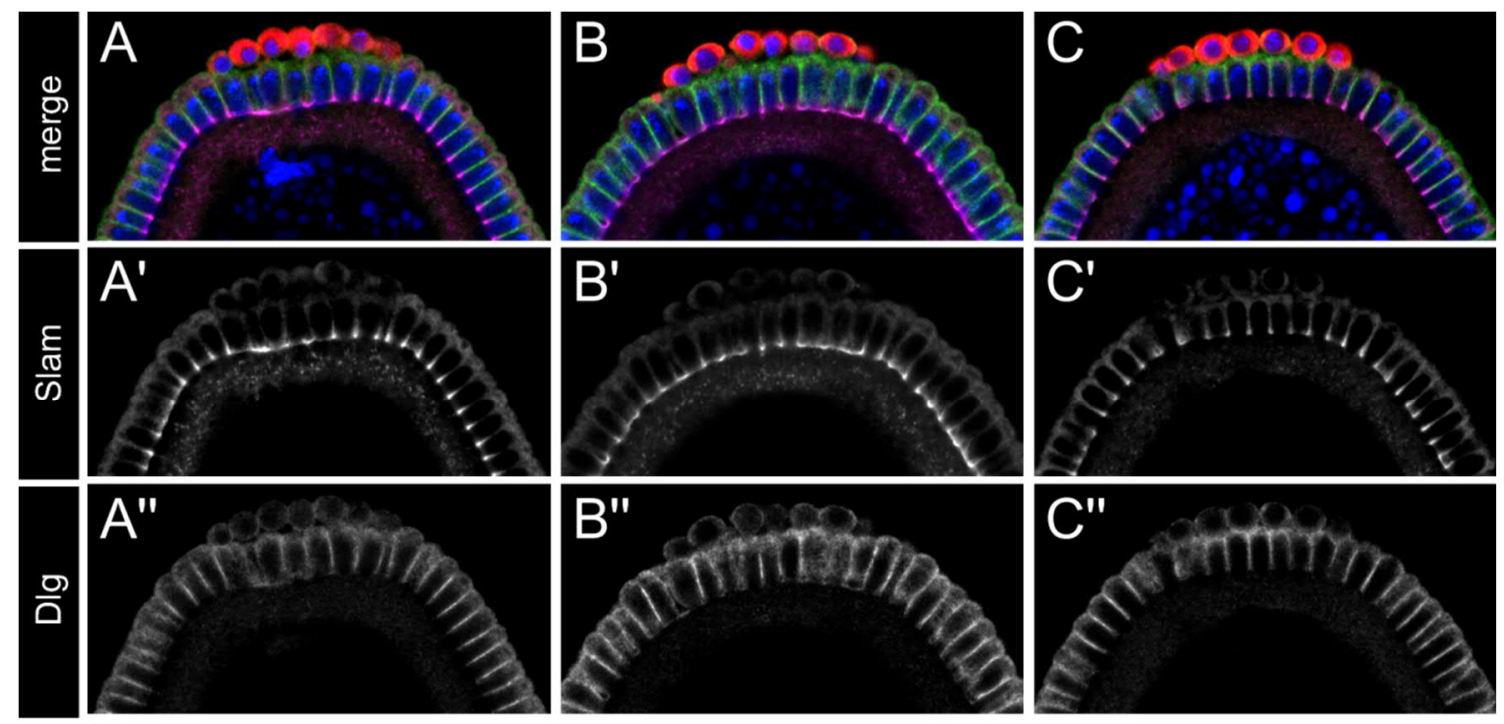

ctrl
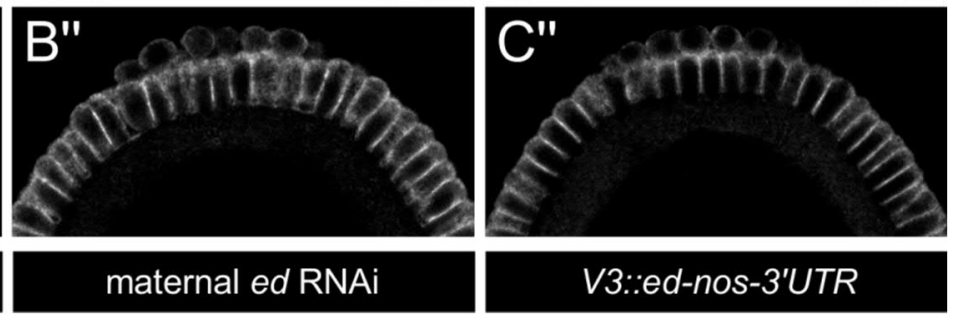

Figure 8: Cellularization is not disturbed in ed RNAi and ed-nos-UTR embryos. (A-C) Confocal sections of posterior poles of stage 5 embryos stained for Slam (magenta), Dlg (green), Vasa (red) and Dapi (blue). Posterior is up. (A) Wild type. Slam (A') localized to the furrow canal. Dlg (A") localizes to lateral membranes. No difference in the localization of Slam or Dlg are found in maternal ed RNAi embryos (B) or ed-nos-3'-UTR embryos (C).

membrane domain (Bilder et al. 2000), Slam is part of the furrow canal (Wenzl et al. 2010), Vasa labels the PGCs and Dapi the nuclei.

Figure 8 shows confocal sections of posterior poles of embryos of the three genotypes mentioned above during cellularization. In all the genotypes Slam localizes to the furrow canal, there is no difference between wild type, maternal ed RNAi or ed-nos-3'UTR (Fig. 8 A',B',C' respectively). Also no difference is found in the localization of Dlg. It always localizes to the lateral membranes of the somatic cells irrespective if Ed protein is present or not. These experiments show that neither the loss of Ed nor its ectopic presence within the PGCs cause cellularization defects, which strongly supports a model that the premature transmigration of PGCs is not indirectly caused by an abnormal cellularization of the PMG. 


\section{An apical actomyosin network depends on the differential expression of Ed}

It has been shown that an intercellular contractile actomyosin cable forms in $e d$ expressing cells at the interface between $e d$-expressing and $e d$ non-expressing cells and that this cable prevents the cell populations from mixing (Wei et al. 2005). It is therefore possible that Ed prevents the PGCs from mixing with the underlying epithelial cells via a similar mechanism. To visualize the actin network fixed embryos have been devitellinized by hand and then stained with Phalloidin to label the actin cytoskeleton, with an anti-Vasa antibody to label the PGCs and with Dapi to show the nuclei. Figure 9 shows confocal sections of posterior poles of wild type, maternal ed RNAi and ed-nos3'-UTR embryos (Fig. 9 A,B,C respectively). In wild type embryos (Fig. 9 A) a weak actin signal can be detected in the cell membranes of the epithelium where it is slightly enriched in the apicolateral membrane domains (Fig. 9 A'). The strong actin signal at

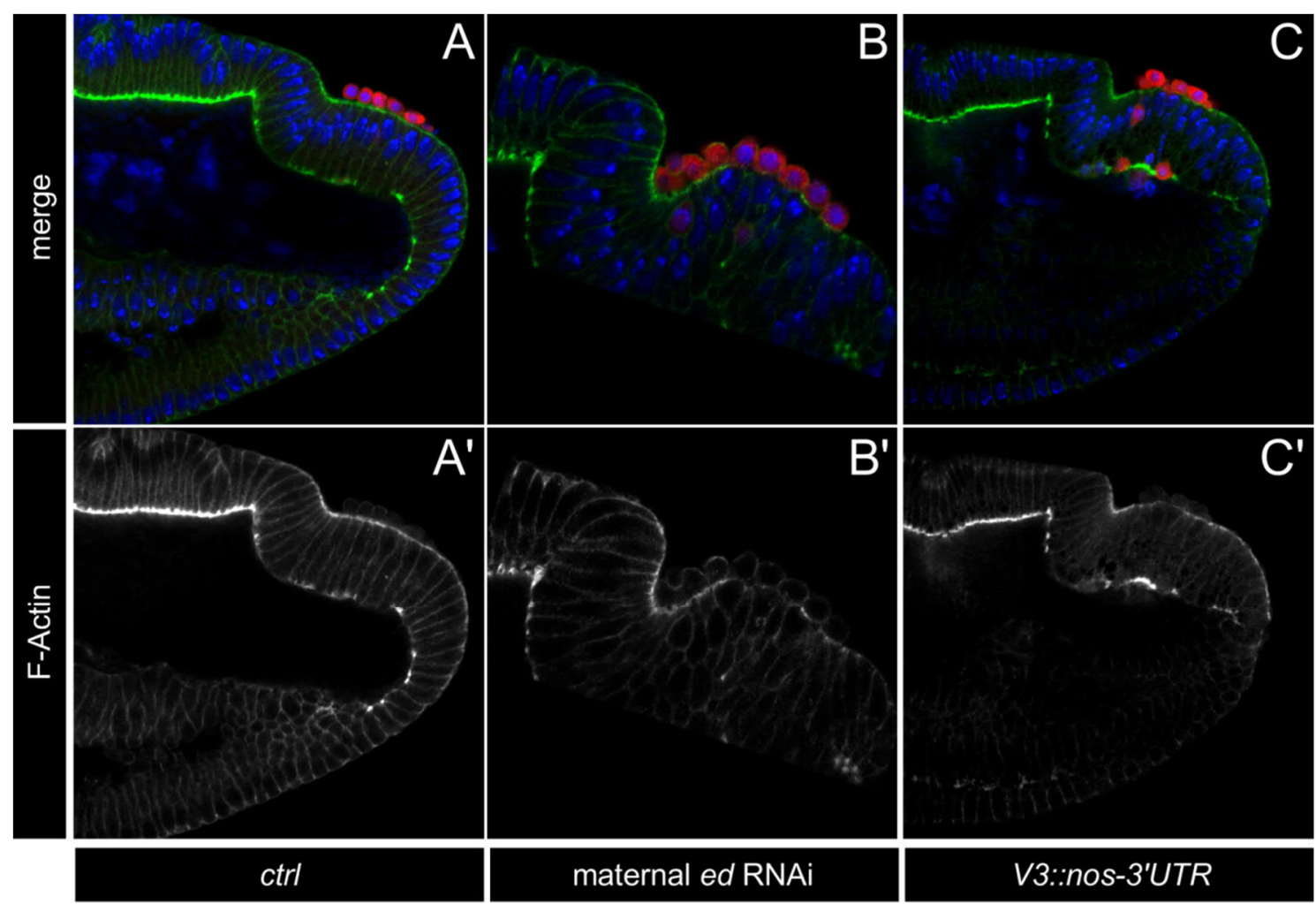

Figure 9: The apical actin network is disrupted in fixed $e d$ RNAi and ed-nos-UTR embryos. (A-C) Confocal sections posterior poles of fixed embryos stained for actin (green), Vasa (red) and Dapi (blue). (A) Wild type embryos show an apical network of actin in the epithelial cells beneath the PGCs (A'). The apical actin network is weakened or disrupted underneath the PGCs in maternal ed RNAi embryos (B) or ed-nos-3'-UTR embryos (C). 


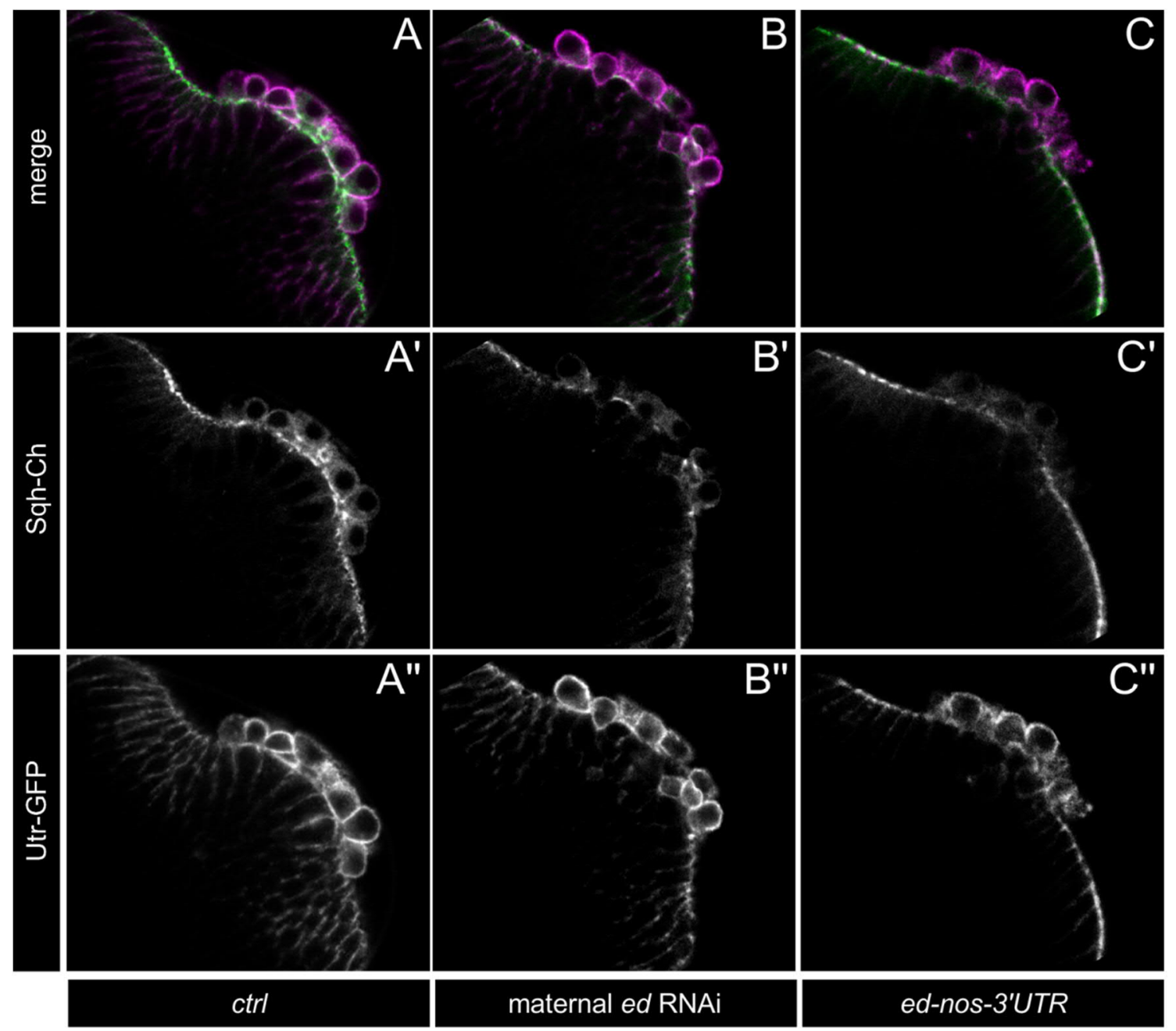

Figure 10: The apical actin network is disrupted in live ed RNAi and ed-nos-UTR embryos. (A-C) Confocal sections of posterior poles of live embryos expressing Utr-GFP (magenta) and Sqh$\mathrm{Ch}$ (green). Utr-GFP labels acting, Sqh-Ch labels myosin-II. In all genotypes Utr-GFP is enriched in the PGCs, but also present in the epithelium (A"-C"). Sqh-Ch is mainly found apically in the epithelial cells $\left(\mathrm{A}^{\prime}-\mathrm{C}^{\prime}\right)$. In control embryos the apical Sqh-Ch signal is uniformly strong at the posterior pole ( $\left.\mathrm{A}^{\prime}\right)$ while in maternal ed RNAI embryos and ed-nos-3'-UTR embryos this apical Sqh-Ch is disrupted or severely weakened (B', C').

the basal side of some epithelial cells represents remnants of the furrow canal. Beneath the PGCs a strong actin signal can be seen in the apical cortex of the epithelium (Fig. 9 $\left.\mathrm{A}^{\prime}\right)$. In maternal ed RNAi or ed-nos-3'-UTR embryos, without an interface between $e d-$ expressing and ed non-expressing cells, this apical actin network is disrupted or severely weakened at this position (Fig. $9 \mathrm{~B}^{\prime}$ and $\mathrm{C}^{\prime}$ ). In contrast, the other features of the actin pattern are indistinguishable from wild type embryos. 
Since the actin cytoskeleton is a highly dynamic structure fixing the samples before analyzing can lead to artifacts. In order to exclude fixation artifacts, live embryos were analyzed which requires the use of transgenic fly lines in which the proteins of interest are tagged with a fluorophore. Here, a transgenic line was used in which the females express Utrophin-eGFP (Utr-GFP) and Spaghetti-Squash-Cherry (Sqh-Ch) in the germline (see methods for details (Rauzi et al. 2010)). Utrophin is a mammalian protein that binds actin fibers and Sqh is the regulatory light chain of myosin-II. Thereby the Utr-GFP signal shows actin filaments while the Sqh-Ch signal highlights contractile actomyosin cables (Rauzi et al. 2010).

Figure 10 shows confocal sections of posterior poles of live stage 6 embryos from mothers either expressing Utr-GFP and Sqh-Ch (ctrl), Utr-GFP, Sqh-Ch and ed RNAi (maternal ed RNAi) or Utr-GFP, Sqh-Ch and ed-nos-3'-UTR (ed-nos-3'UTR). In all genotypes Utr-GFP is enriched in the PGCs but can also be detected apically in the somatic cells (Fig. 10 A",B",C", respectively). Sqh-Ch, in contrast, is highly enriched in the apical cortex of the epithelial cells and barely present in the PGCs (Fig. 10 A',B', C' respectively). In control embryos the apical Sqh-Ch signal is uniformly strong at the posterior pole, also beneath the PGCs (Fig. 10 A') while in embryos which lack an interface between $e d$-expressing and $e d$ non-expressing cells this apical Sqh-Ch signal is disrupted or severely weakened (Fig. 10 B' and $C^{\prime}$ ).

These findings show that wild type embryos have an apical actomyosin network in the epithelial cells beneath the PGCs and that this actomyosin network is disturbed in the absence of the interface between $e d$-expressing and $e d$ non-expressing cells.

Taken together, here, it was shown that Ed, a homophilic cell adhesion molecule, is expressed in the somatic epithelial cells but not in the PGCs during early embryogenesis. If Ed is removed from the soma the PGCs start to traverse the underlying epithelium already shortly after cellularization. Since this phenotype can also be observed when ed is ectopically expressed within the PGCs in addition to its expression in the epithelial cells the untimed transmigration is not due to Ed functioning as a normal cell adhesion factor. This rather demonstrates that the interface between $e d$-expressing and $e d$ nonexpressing cells is crucial to avoid untimed migration of the PGCs. 
These interfaces and their biological relevance have been studied before in other contexts like dorsal closure. It was shown that the differential expression of $e d$ is sufficient to trigger the formation of an actomyosin cable within the ed expressing cells at the interface.

Here, it was shown that in wild type embryos an apical meshwork of actomyosin is present in the epithelial cells beneath the PGCs. This actomyosin network is weakened or disrupted if Ed is removed from the epithelium or ectopically expressed within the PGCs. 


\section{Lost}

\section{Lost is a sponge body component and ubiquitously present in early}

$\underline{\text { embryos }}$

It was reported earlier that Lost can be found in the sponge bodies during oogenesis (Snee and Macdonald 2009). To verify this, ovaries from females which have a GFP enhancer trap inserted into the lost locus have been analyzed. In this fly line a Lost-GFP fusion protein is expressed instead of the wild type protein (Morin et al. 2001). Figure 11 A shows a confocal section of an ovariole expressing Lost-GFP (green), stained with Dapi (blue) and Phalloidin (red). Figure 11 B shows a different optical section of the same egg chamber highlighting the developing oocyte. Lost-GFP can be detected from mid-oogenesis onwards. It is present in large perinuclear structures around the nurse cell nuclei and highly enriched in the oocyte.

In order to analyze the localization of Lost protein in early embryos fixed wild type preblastoderm embryos were stained with a specific antibody against Lost (Graf 2007).
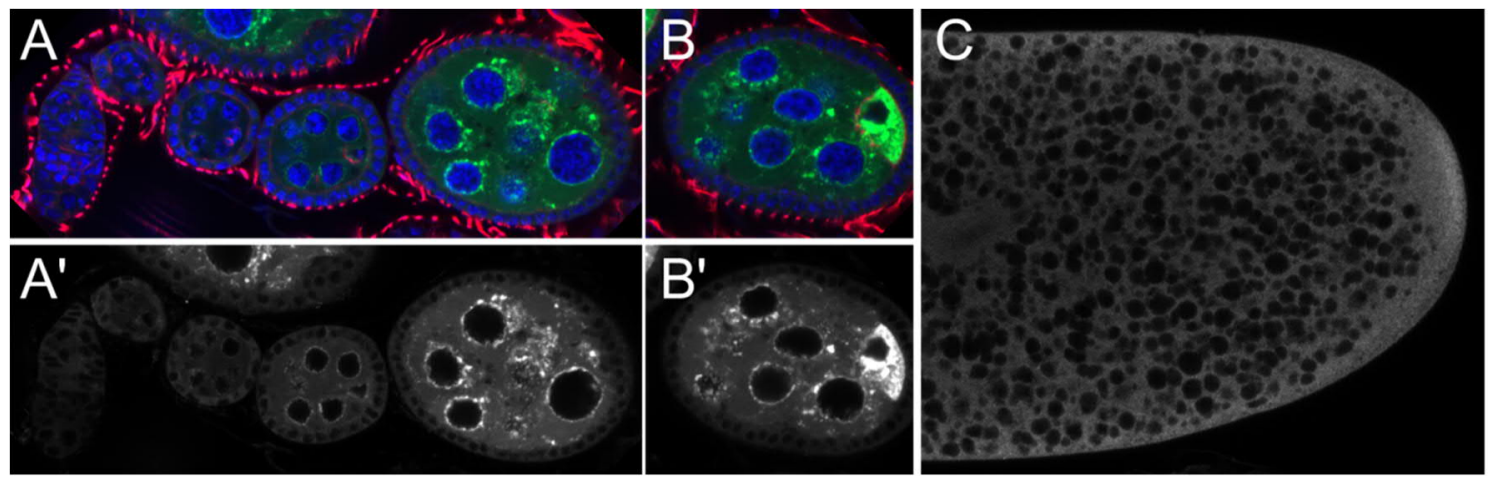

Figure 11: Lost is a sponge body component and ubiquitously present in early embryos. (A) Confocal section of an ovary expressing Lost-GFP (green), stained with Phalloidin (red) and Dapi (blue). (B) Different confocal section of the rightmost egg chamber in (A) showing the developing oocyte. (A') In the nurse cells Lost-GFP is enriched in perinuclear patches resembling nuage. (B') Lost-GFP is accumulating in the developing oocyte. (C) Confocal section of the posterior half of a preblastoderm wild type embryo stained for Lost. Lost is present ubiquitously in the embryo and slightly enriched at the posterior pole. 
Figure $11 \mathrm{C}$ shows a confocal section of the posterior half of an early wild type embryo. Lost protein can be detected throughout the embryo. It is slightly enriched at the posterior pole.

\section{PGC development is impaired in maternal lost mutant embryos}

In wild type embryos the vast majority of PGCs transmigrates during stages 9 and 10 of development through the PMG whereas in maternal lost mutants an increased number of PGCs cross the epithelium already during stages 6 and 7 (Hertel 2011). Here the transmigration phenotype was reanalyzed and quantified (Fig. 12). Fixed embryos of the desired developmental stages were stained with an antibody against Vasa protein to label the PGCs. Then 3D confocal stacks of the posterior pole were acquired and analyzed. Using the cell counter plugin from Fiji the total number of PGCs was counted as well as the number of transmigrating PGCs. In wild type embryos only a small number of PGCs transmigrate during stages 6 and 7 (Fig. 12 A). In embryos maternally mutant for lost the number of transmigrating PGCs is drastically increased (Fig. 12 B). The same phenotype can be observed in maternal knockdown of lost using RNAi (Fig. $12 \mathrm{C})$. In control embryos on average 6 PGCs cross the epithelium during stages 6 and 7, while in lost mutants or lost RNAi that number is increased to 11 or 14 , respectively. The difference is statistically significant (Mann-Whitney-U test, $p<0.001$ ). Since the total number of PGCs varies between different embryos of the same genotype as well as between genotypes the number of transmigrating PGCs was also normalized to the total number of PGCs in that embryo. This reveals that in control embryos on average $13 \%$ of PGCs transmigrate, compared to $22 \%$ in lost mutants and $35 \%$ in lost RNAi. These differences are statistically significant (Mann-Whitney-U test, $* * \mathrm{p}<0.01$ and $* * *$ $\mathrm{p}<0.001$ respectively).

A general feature of developing PGCs is their transcriptional quiescence (Leatherman and Jongens 2003). This has been reported for the development of mice, worms, flies and others (Nakamura and Seydoux 2008, Strome and Updike 2015). The transcriptional silencing in the different model organism is achieved by different 

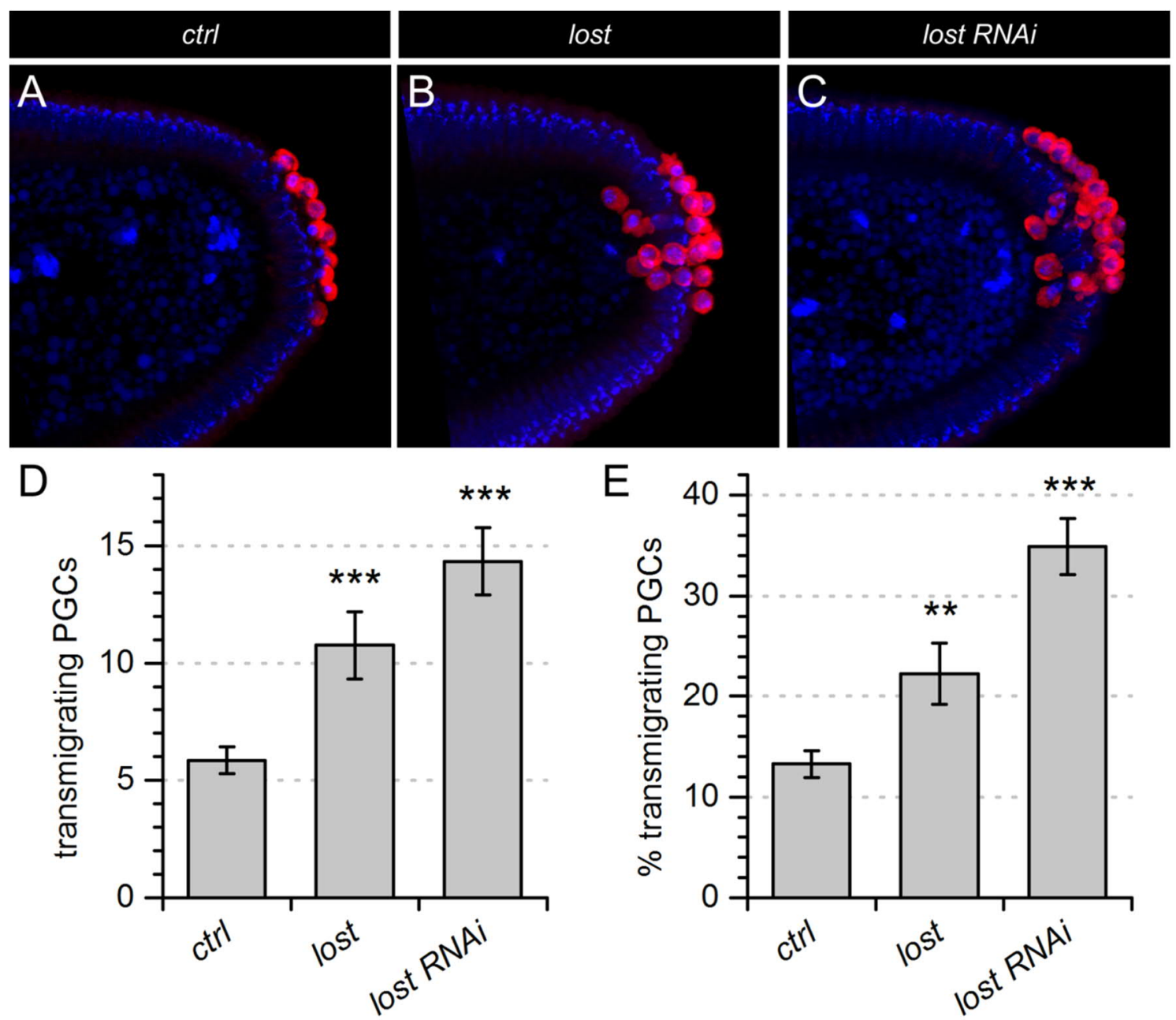

Figure 12: The PGCs in embryos which lack maternal lost cross the PMG epithelium prematurely. (A-C) Maximum intensity projections of 2-5 confocal sections of posterior poles of stage 6 embryos stained for Vasa (red) and Dapi (blue). (A) In wild type embryos the PGCs reside at the posterior pole. (B,C) In lost embryos or lost RNAi embryos PGCs migrate through the epithelium. (D,E) Quantification of the premature transmigration phenotype. The columns show the average total number (D) or relative number (E) of transmigrating PGCs during stages 6 and 7 of the three genotypes. Error bars show SEM. *** $p<0.001$. ** $p<0.01$ Mann-Whitney-U test.

mechanisms but it is always crucial to their development. If the transcriptional silencing is disturbed in Drosophila the PGCs show developmental defects and eventually die (Nakamura et al. 1996).

During early Drosophila embryogenesis the nuclei are transcriptionally active. After PGC budding the somatic nuclei remain transcriptionally active while the PGC nuclei become transcriptionally silent (Seydoux and Dunn 1997). Later, when the PGCs start their active migration at stage 9, they become transcriptionally active (Zalokar 1976). 

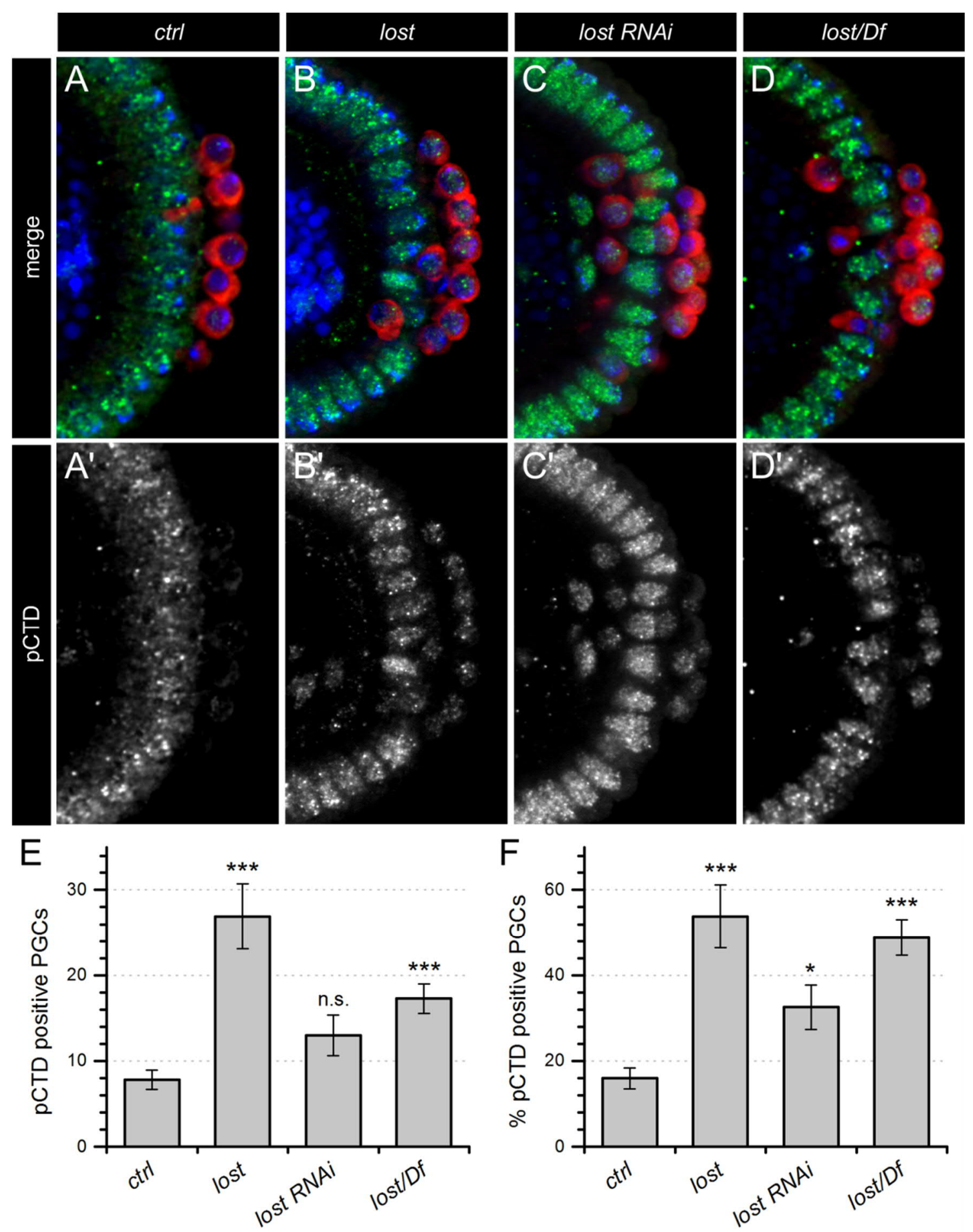

The major DNA-dependent RNA polymerase for protein coding genes, RNApolymerase II, has a characteristic 7 amino acid repeat at its $\mathrm{C}$-terminal domain (CTD) which is conserved across all phyla (Dahmus 1996). The phosphorylation state of these repeats correlates with the activity of the enzyme. It is only fully phosphorylized while 
Figure 13: The PGCs in embryos which lack maternal lost show ectopic transcriptional activity. (A-D) Maximum intensity projections of 2-5 confocal sections of posterior poles of stage 6 embryos stained for Vasa (red), pCTD (green) and Dapi (blue). The presence of a pCTD signal indicates transcriptional activity. (A) In wild type embryos only the somatic cells but not the PGCs show a strong pCDT signal (A'). (B-D) In embryos which lack the maternal lost contribution many PGCs show a clear pCTD signal (B'-D'). The pCTD signal of the somatic nuclei is considerably stronger. (E,F) Quantification of the ectopic transcriptional activity of the PGCs phenotype. The columns show the average total number (D) or relative number (E) of pCTD-positive PGCs in stage 6 embryos of the four genotypes. Error bars show SEM. $* * * p<0.001$. $* * \mathrm{p}<0.01 . * \mathrm{p}<0.05$. n.s. not significant, Mann-Whitney-U test.

actively elongating mRNA. The phosphorylation state and by that transcriptional activity can be readily detected using immunofluorescence (Palancade and Bensaude 2003).

Figure 13 shows the posterior poles of stage 6 embryos of wild type (A,A'), maternal lost mutants (B, $\left.\mathrm{B}^{\prime}\right)$, maternal lost $\mathrm{RNAi}\left(\mathrm{C} \mathrm{C}^{\prime}\right)$ and embryos from mothers which are heterozygous for lost and a deficiency line covering the lost locus stained with antibodies against pCTD (green), Vasa that labels the PGCs (red) and Dapi to show the nuclei (blue).

In wild type embryos the PGCs are mostly transcriptionally silent, as shown by the absence of pCTD signal in the PGCs (Fig. 13 A,A'). In maternal lost mutant embryos the number of PGCs which are transcriptionally active is greatly increased (Fig. 13 B,B') revealed by a strong nuclear signal of pCTD in the PGCs. In maternal lost RNAi embryos the PGCs also show transcriptional activity, although to a lesser extent than in lost embryos (Fig. 13 C,C'). Embryos from lost/Df mothers show the same ectopic transcription in the PGCs (Fig. 13 D,D'). The strength of this phenotype can be quantified by counting the number of transcriptionally active PGCs using the Fiji Cell Counter plugin. On average the absolute number of transcriptionally active PGCs is 8 for wild type, 26 for lost mutants, 14 for maternal lost RNAi and 17 for lost/Df (Fig. 13 E). Besides lost RNAi the increased numbers in ectopic transcription are highly statistically significant (Mann-Whitney-U test, $\mathrm{p}<0.001)$ compared to wild type. Since the total number of PGCs can differ between the individual embryos and between the genotypes the relative number of transcriptionally active PGCs was calculated (Fig: 13 
F). In wild type embryos approximately $16 \%$ of the PGCs show transcriptional activity during stage 6, in maternal lost mutants 56\%, in lost RNAi 32\% and in lost/Df 50\%. These changes are statistically significant compared to wild type with $\mathrm{p}<0.05$ for lost RNAi and $\mathrm{p}<0.001$ for lost and lost/Df.

This demonstrates that in embryos that come from mothers which lack lost function the PGCs show ectopic transcriptional activity during embryonic stage 6.

\section{Cellularization defects at posterior pole in embryos lacking maternal lost}

During stages 9 and 10. when most of the PGCs leave the midgut pocket in wild type embryos, the PMG epithelium undergoes an epithelium-to-mesenchymal transition (EMT) which weakens the adhesion between the cells and allows the PGCs to pass (Seifert and Lehmann 2012). The presence of the premature transmigration phenotype in maternal lost mutants (see Fig. 12) therefore raises the question whether Lost functions during cellularization or in cell adhesion. Several proteins show characteristic localization patterns during cellularization and in the epithelium. Their localization indicates a proper formation of the epithelium during stage 5.

Bazooka (Baz) labels the apical part of the lateral membrane of epithelial cells (Tepass 2012). The lateral membrane domain contains Discs-large (Dlg), a protein which among others has a role in maintaining apicobasal cell polarity and cell adhesion (Bilder et al. 2000). During cellularization the invaginating membrane front, the furrow canal, contains Slam, which recruits DRhoGEF2 and by that promotes the formation of contractile actomyosin at the invaginating membrane (Wenzl et al. 2010). During cellularization the centrosomes are located apically to the somatic nuclei. The microtubules extent basally and form a very regular, cage-like structure around the nuclei (Warn and Warn 1986).

Therefore, the localization pattern of the four before mentioned proteins was analyzed in fixed stage 5 embryos. They were visualized using immunofluorescence and 3D confocal image stacks were acquired of a lateral part of the embryo (lat.) or the posterior pole (post.) showing the forming epithelium. The analyzed areas are indicated in Figure 
Figure 14: Cellularization phenotypes in lost embryos are restricted to the very posterior pole. (A) Confocal section of a wild type embryo stained for Vasa (red), Slam (green) and Dapi (blue). The yellow boxes highlight the regions of interest: Posterior (post.), beneath the PGCs and lateral (lat.), a lateral part of the embryo, distant from the posterior pole. (B-Q) Confocal sections of wild type or lost stage 5 embryos stained for Baz, Dlg, Slam or Tubulin. The pictures showing the two regions of interest are always from the same embryo for a given genotype and staining. (B,C) In wild type embryos Baz localizes to the apical membrane, independent of the region. (D,E) In lost embryos Baz localizes wild type-like in the lateral regions, while beneath the PGCs it is also found in the furrow canal. $(\mathrm{F}, \mathrm{G})$ In wild type embryos $\mathrm{Dlg}$ localizes to the lateral membrane, independent of the region. (H,I) In lost embryos Dlg localizes wild type-like in the lateral regions, while beneath the PGCs it is sometimes also found in the furrow canal or completely missing at a lateral membrane. $(\mathrm{J}, \mathrm{K})$ In wild type embryos
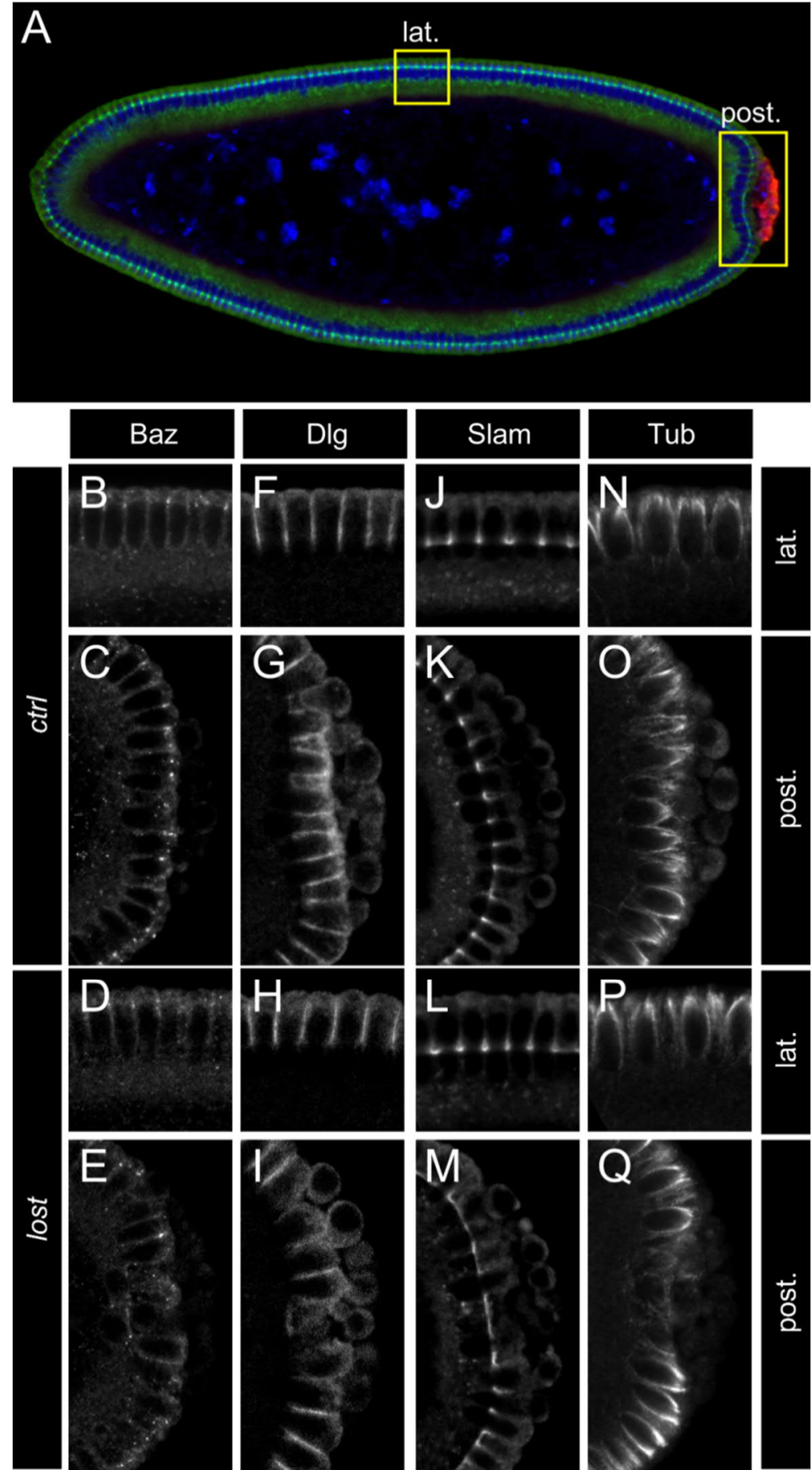

Slam localizes to the furrow canal, independent of the region. (L,M) In lost embryos Slam localizes wild type-like in the lateral and posterior regions. The furrow canal can be malformed and enlarged, spanning several nuclei. (N,O) In wild type embryos Tubulin forms regular cage-like structures around the nuclei, independent of the region. (P,Q) In lost embryos the Tubulin pattern is wild typelike in the lateral regions. Beneath the PGCs the Tubulin cages are missing or malformed.

14 A. The lateral and the posterior confocal sections always show fractions of the same embryo for a given genotype and protein stained. In wild type embryos Baz is localized 
in apical puncta at the cell interfaces in the lateral part of the embryo (Fig. 14 B) as well as at the posterior pole beneath the PGCs (Fig. 14 C). In embryos from lost mutant mothers Baz shows a normal localization in lateral portion of the embryo (Fig. 14 D) while at the posterior pole ectopic Baz can be detected in the furrow canal (Fig. 14 E). The apicolateral Baz puncta are sometimes absent.

Dlg localizes to the lateral membrane basally to Baz at cell interfaces in wild type embryos with no difference between the lateral and posterior portion of the embryo (Fig. 14 F,G respectively). In maternal lost mutant embryos Dlg localizes normally in the lateral epithelium (Fig. $14 \mathrm{H}$ ) whereas at the posterior pole Dlg can sometimes be found in the basal domain of the membrane. Also at some cell interfaces Dlg is largely absent (Fig. 14 I).

Slam, which marks the furrow canal, is only detected there in wild type embryos (Fig. $14 \mathrm{~J}, \mathrm{~K})$ as well as in maternal lost mutants both in the lateral portion of the embryo and at the posterior pole (Fig. 14 L,M). However, in lost mutants the furrow canal sometimes is malformed and severely enlarged spanning several nuclei (Fig. 14 M). This phenotype only occurred beneath the PGCs at the posterior pole but never in lateral portion of the embryo.

The very regular pattern of microtubules is readily visible in the lateral parts of the embryo for both wild type and maternal lost mutants (Fig. 14 N,P). At the posterior pole beneath the PGCs the same regular pattern can be seen in wild type embryos (Fig. 14 O) while in maternal lost mutants this pattern is disrupted (Fig. 14 Q). The tubulin "cages" around the nuclei can be severely malformed, reduced or absent. This tubulin phenotype is only present beneath the PGCs.

lost mRNA is maternally provided and the protein is ubiquitously present in early embryos with a slight enrichment at the posterior pole (see Fig. $11 \mathrm{C}$ ). In general cellularization and cell polarity are not affected in maternal lost mutants in the most parts of the embryo. Only at the posterior pole, right underneath the PGCs, membrane polarity markers Baz and Dlg are mislocalized or absent, the furrow canal is malformed and the tubulin pattern is disturbed or absent. Since the Lost protein is present throughout the entire embryo a general role of Lost in cellularization seems to be unlikely. 


\section{The posterior somatic nuclei have less transcription in maternal lost}

$\underline{\text { mutants }}$

It has been shown that zygotic transcription of several factors is essential for cellularization, including Slam (Edgar et al. 1986, Acharya et al. 2013). In embryos from lost mutant mothers the amount of tubulin at the posterior pole can be severely reduced (Fig. 14 Q) alongside with other defects in cellularization (Fig. 14).

One possible cause for that could be reduced zygotic transcription from the somatic nuclei underneath the PGCs (hereafter called SNUPs). The overall transcriptional activity can be easily detected by an antibody against the phosphorylated carboxyterminal domain (pCTD) of RNA-polymerase II. To assess the possibility of reduced levels of transcription at the posterior pole fixed embryos were stained with an antipCTD antibody to label active transcription, an anti-Vasa antibody to label the PGCs and Dapi to show the nuclei.

Figure 15 A shows the posterior pole of an embryo from a wild type mother. A clear and strong pCTD signal is detected in all somatic nuclei (Fig. 15 A') indicating a uniformly high level of active transcription. In embryos from lost mutant mothers the pCTD signal in the SNUPs is weaker compared to somatic nuclei which are not directly beneath the PGCs (Fig. 15 B,B'). The nuclei are present as shown by the Dapi staining (Fig. 15 B"). The same phenotype can be found in embryos from mothers expressing lost RNAi (Fig. 15 C,C',C") and in embryos from lost/Df mothers (Fig. 15 D,D',D").

The magnitude by which transcription is reduced in the SNUPs can be estimated by comparing the signal intensity of the pCTD staining within the SNUPs to the signal intensity within the other somatic nuclei. For that, 3D confocal stacks of posterior poles of stage 4 embryos stained for pCTD, Vasa and Dapi of the respective genotypes were recorded. A Fiji macro was created for batch-processing each image stack individually (see Methods for details). In brief for each slice in a 3D stack a mask was created from the Dapi channel and the PGC nuclei were removed from that mask resulting in a selection of only the somatic nuclei. The part of that mask which lies beneath a Vasa signal was detected and defined as SNUP (= somatic nuclei beneath PGCs). The total signal intensity in the pCTD channel and the number of analyzed voxels was then 

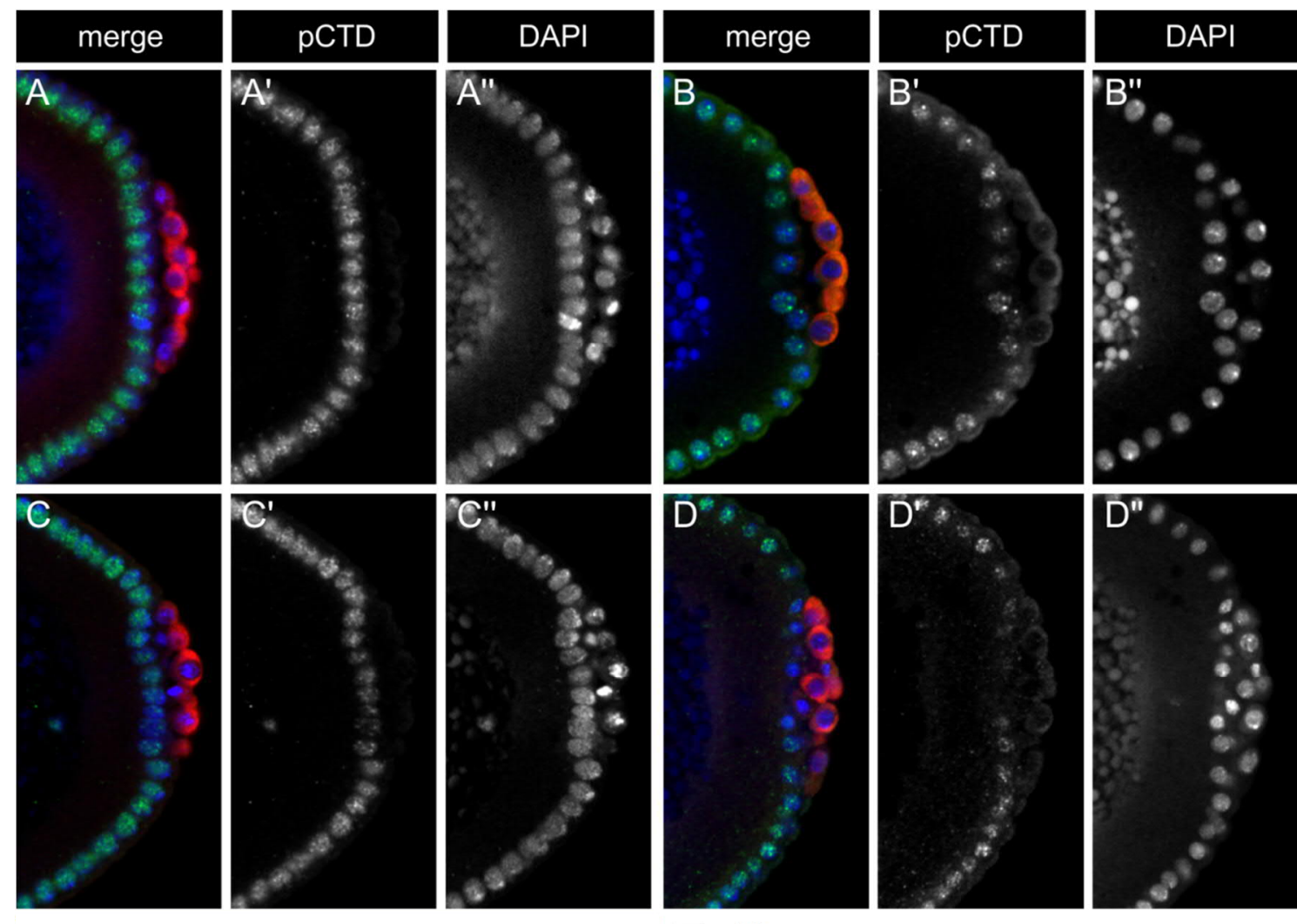

Figure 15: The somatic nuclei underneath the PGCs (SNUPs) have reduced levels of transcription in embryos which lack maternal lost. (A-D) Confocal sections of posterior poles of stage 4 embryos stained for Vasa (red), pCTD (green) and Dapi (blue). (A) In wild type embryos the pCTD signal is uniformly strong in all somatic nuclei. (B-D) In embryos which lack maternal lost the pCTD signal in the SNUPs is weaker than that of the
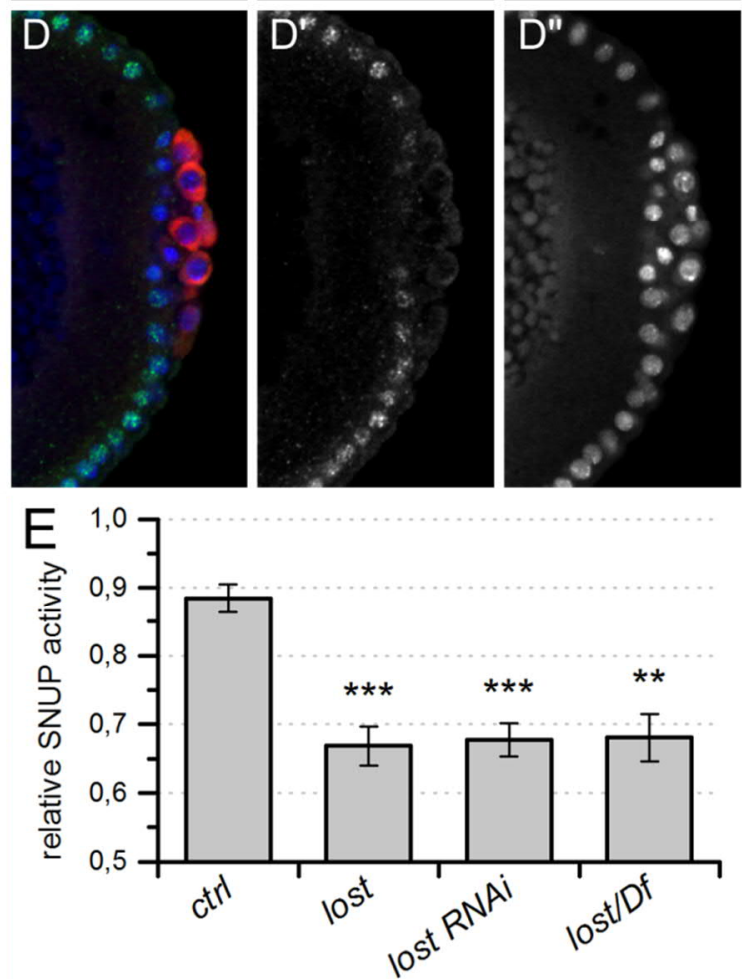
other somatic nuclei. (E) Quantification of the reduced levels of transcription. The bars show the average relative intensity of the pCTD signal of the SNUPs compared to the other somatic nuclei of the same embryo for the indicated genotypes. Error bars show SEM. *** p<0.001. ** $\mathrm{p}<0.01$ MannWhitney-U test.

measured in the SNUP area and outside the SNUP area of the Dapi mask. From that the average pCTD signal intensity within the SNUPs and within the other somatic nuclei was calculated. By that method the complete posterior pole of an embryo is quantified and the relative transcriptional activity of the SNUPs compared the rest of the soma is obtained. The analysis of the data revealed that even in control embryos the transcriptional activity of the SNUPS is only $88 \%$ of that in the rest of the soma (Fig. 15 E). In embryos from mothers which are devoid of Lost function the transcriptional 
activity in the SNUPs is reduced to $67 \%$ in maternal lost mutants, $68 \%$ in maternal lost RNAi and $68 \%$ in embryos from lost/Df mothers (Fig. 15 E). These differences are highly statistically significant when compared to control embryos (Mann-Whitney-U test; *** $\mathrm{p}<0.001 ; * * \mathrm{p}<0.01)$.

While the vast majority somatic nuclei of wild type stage 4 embryos shows a uniformly strong transcriptional activity the SNUPs show slightly reduced levels of transcription. In embryos from mothers who lack Lost function however the transcriptional activity of the SNUPs is reduced to two thirds of that of the rest of the soma.

\section{Fluorescent in-situ hybridyzation reveals localization patterns of germ} plasm RNAs

Lost protein is present throughout the entire early embryo (Fig. $11 \mathrm{C}$ ). However, all the lost mutant phenotypes (premature transmigration of PGCs (Fig. 12), ectopic transcription in PGCs (Fig. 13), cellularization defects (Fig. 14) and reduced levels of transcription in SNUPs (Fig. 15)) involve PGCs or are restricted to the very posterior pole of the embryo. Furthermore, Lost has been reported to be part of several RNAprotein complexes (Herold et al. 2009, Snee and Macdonald 2009) as well as being involved in the accumulation of germ plasm in the oocyte during the last stages of oogenesis (Sinsimer et al. 2011) and being potentially an RNA binding protein (Marchler-Bauer et al. 2017). This raises the question whether the germ plasm RNAs are properly localized in maternal lost mutant embryos.

The germ plasm is composed of several proteins (for example Vasa and Osk) and different RNAs (reviewed by Santos and Lehmann (2004a)). Especially gcl, cycB, osk, nos and pgc RNAs have been extensively studied and their posterior localization in early embryos is well established. Fluorescent in-situ hybridization (FISH) was used to visualize the localization of these RNAs (see methods for details). In brief fixed embryos of stages 1 or 2 were probed with a labeled antisense-RNA against the respective target RNA. The antisense-RNA probe was then detected by a horseradish peroxidase (HRP)-coupled antibody which in turn produces a fluorescent signal when 


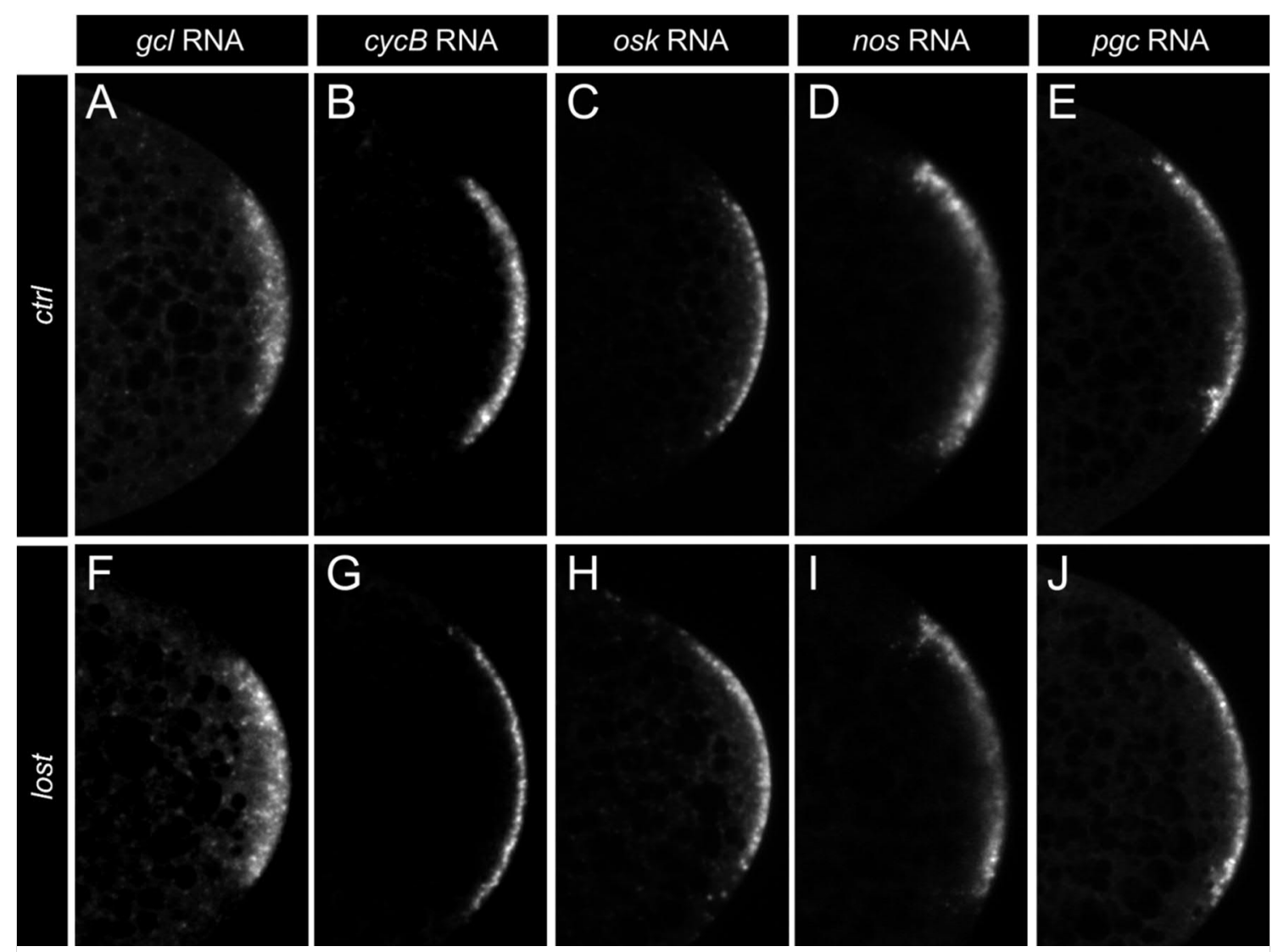

Figure 16: The localization patterns of germ plasm RNAs revealed by FISH. (A-J) Confocal sections of posterior poles of preblastoderm embryos stained for the indicated RNAs by fluorescent in-situ hybridization (FISH). $g c l, c y c B$, osk, nos and $p g c$ RNA localize to the germ plasm at the posterior pole. There is no difference between wild type (A-E) and lost embryos (F-J). Note that the staining intensity at the very posterior tip of the embryo seems lower than at the margins of the germ plasm for nos and $p g c$ RNA independent of the genotype (D,E,I,J).

subjected to a tyramide signal amplification (TSA) reaction. The stained embryos are then analyzed by confocal microscopy.

Figure 16 shows the localization of $g c l(\mathrm{~A}, \mathrm{~F}), \operatorname{cycB}(\mathrm{B}, \mathrm{G}), \operatorname{osk}(\mathrm{C}, \mathrm{H}), \operatorname{nos}(\mathrm{D}, \mathrm{I})$ and $p g c$ RNA (E,J) at the posterior poles of wild type embryos (A-E) and embryos from lost mutant mothers (F-J). All analyzed RNAs show a typical germ plasm localization at the posterior cortex as reported in the literature (Lecuyer et al. 2007) in both wild type and lost embryos. However, nos and $p g c$ FISH stainings differ in one aspect from $g c l, c y c B$ and $o s k$ : The staining intensity at the very posterior tip of the embryo seems lower than at the margins of the germ plasm (Fig. 16 D,E), although the amount of germ plasm RNAs is reported to be the highest in that region (Slaidina and Lehmann 2017). This 
phenomenon is highly reproducible, independent of genotype (compare Fig. 16 D and I) and only occurring when staining nos or $p g c$ RNA.

\section{Comparing germ plasm RNA staining patterns using FISH intensity profiles}

To visualize and quantify the distribution of germ plasm RNAs the embryos were counterstained with an anti-Vasa antibody to label the germ plasm. Then 3D confocal stacks of posterior poles of stage 1 or 2 embryos were acquired covering the complete germ plasm. Figure 17 show y-projections of complete confocal stacks from wild type $\left(\mathrm{A}^{\prime}, \mathrm{A}^{\prime \prime}\right)$ and maternally lost mutant embryos $(\mathrm{B}, \mathrm{B} ")$. In this projection the germ plasm RNAs (Fig. 17 A',B') as well as Vasa protein (Fig. 17 A",B") form a circular disc at the posterior pole of the embryo. To quantify the distribution of germ plasm RNAs and Vasa protein intensity profiles along a straight line through the middle of the germ plasm were obtained (see blue and red lines in Fig. 17 A,B). A Fiji macro was created for batch processing each 3D confocal stack (see methods for details). The intensity profiles for a given genotype and staining (germ plasm RNA FISH or Vasa protein) were then scaled to the diameter of the germ plasm and normalized to highest intensity. This allows averaging the intensity profile curves from a given genotype and staining and comparison between genotypes.

Figure 17 shows the averaged intensity profiles of $g c l, c y c B$, osk and nos RNA (C-F) and the corresponding Vasa protein (G-J) for wild type embryos (blue) and maternal lost mutant embryos (red). In all analyzed conditions the Vasa protein always forms a circular disk with a uniform distribution (Fig. 17 A",B"). This is reflected by the averaged intensity profiles (Fig. 17 G-J): A very low background signal between 0.05 (Fig. $17 \mathrm{I}$ ) and 0.2 (Fig. $17 \mathrm{H}$ ) of the maximum intensity, followed by a steep increase which reaches a plateau at 0.8 to 0.9 and a steep decrease to background levels. Regarding the distribution of Vasa protein in the germ plasm no difference between wild type embryos and maternally lost mutant embryos could be detected. The intensity profiles of $g c l, c y c B$ and $o s k$ RNA show the same features as those from Vasa protein. 

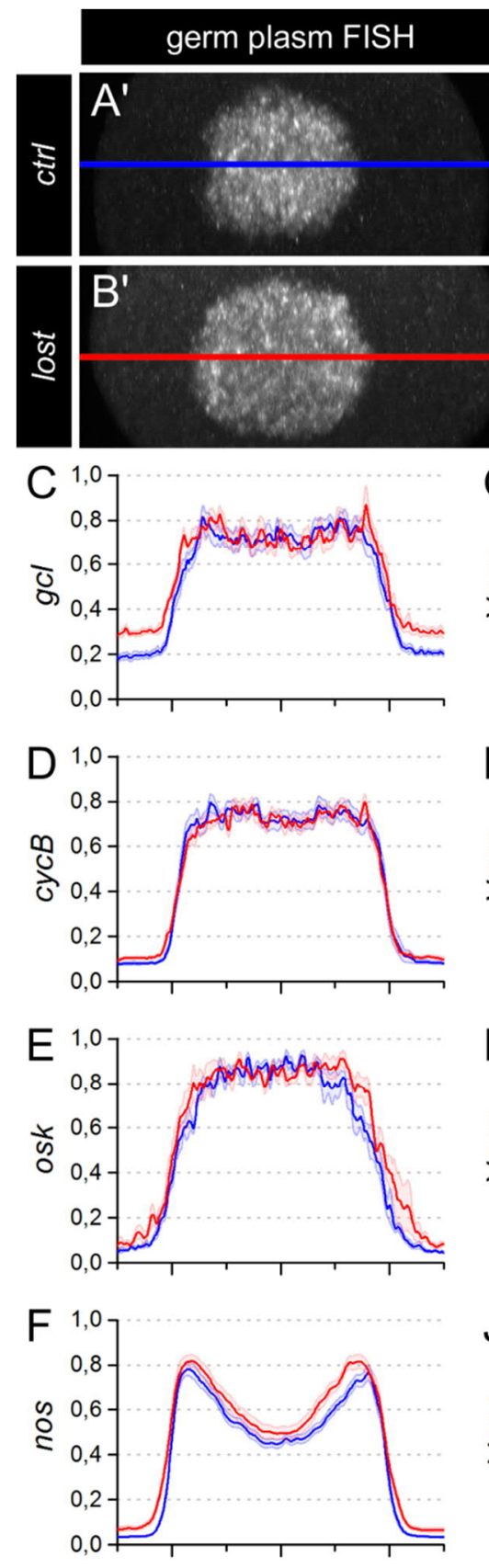

relative germ plasm position
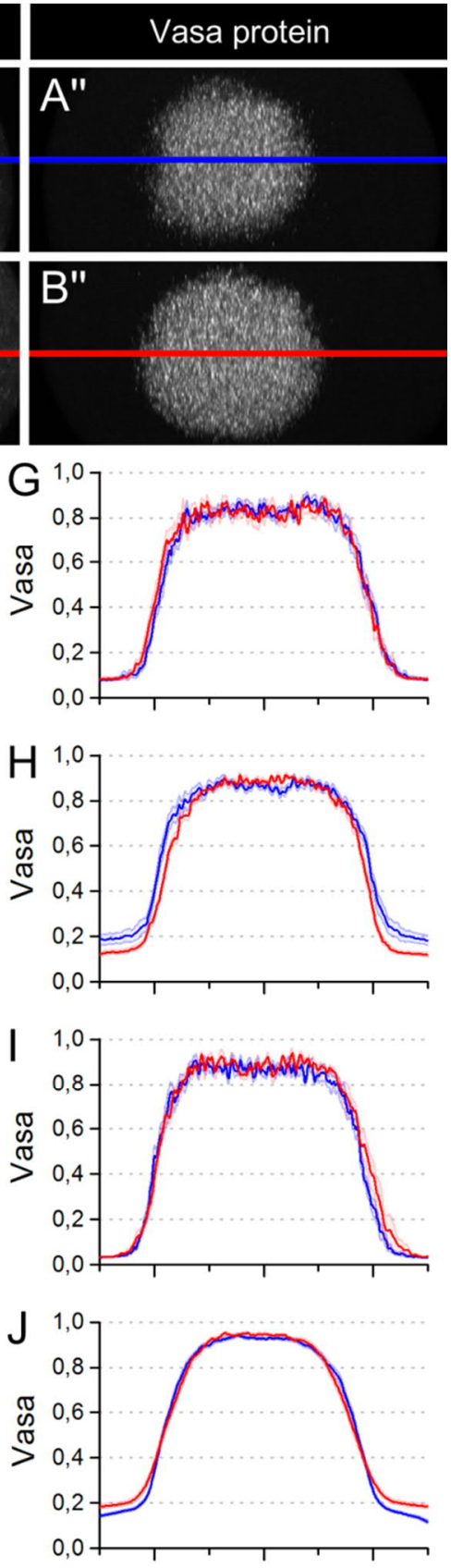

relative germ plasm position
Figure 17: The localization patterns of most germ plasm RNAs do not differ between wild type and lost. (A,B) yprojections of confocal stacks covering the complete germ plasm stained for a germ plasm RNA and Vasa protein. (C-J) Average intensity profiles of germ plasm RNAs (C-F) and the corresponding Vasa channel (G-J) from control (blue) and maternal lost embryos (red) along a straight line through the center of the germ plasm (see red and blue lines in $(\mathrm{A}, \mathrm{B}))$ in y-projections of confocal stacks covering the complete germ plasm stained for a germ plasm RNA and Vasa protein. The shaded areas show the SEM. The intensity profile curves of $g c l$ (C), $c y c B$ (D) and osk (E) have the same shape as their corresponding Vasa profiles (G-I): A steep incline, followed by a plateau and a sharp decline. The intensity profile of nos (F) shows a depression reflecting the lower FISH staining intensity in the center of the germ plasm (see Fig. 16 D,I). There is no difference in the average intensity profiles of $g c l, c y c B$, osk and nos between control and maternal lost mutant embryos.

A uniformly high staining intensity within the germ plasm (Fig. 17 C-E) can be detected for both wild type (blue) and maternal lost mutant (red) embryos. The before mentioned reduction in staining intensity at the very posterior pole for nos RNA (Fig. 16 D,I) is clearly reflected in the intensity profile (Fig. 17 F). The margins of the germ plasm show a high intensity ( 0.8 of the maximum), comparable to the other RNAs. However, 
this intensity is reduced to 0.5 in the center of the germ plasm which results in a clear dent in the intensity profile. There is no difference between wild type (blue) and maternal lost mutant embryos (red).

\section{The $p g c$ FISH intensity profile is altered in maternal lost embryos}

The distribution of $p g c$ RNA in the germ plasm of wild type embryos shows similar features as the one for nos RNA: The staining intensity in the margins of the germ plasm (labeled by Vasa (Fig. 18 A")) is considerably higher than in its center (Fig. 18 $\left.\mathrm{A}^{\prime}\right)$. This phenomenon is highly reproducible and thereby reflected in the averaged intensity profile (Fig. $18 \mathrm{C}$, blue). As for nos RNA (see Fig. $17 \mathrm{~F}$ ) the mean intensity in the center of the germ plasm is around 0.4 while the margins reach a normalized intensity of 0.8 . Vasa protein is uniformly distributed (Fig. 18 A") which results in an in intensity profile with an even plateau at 0.9 (Fig. $18 \mathrm{D}$, blue). In embryos from lost mutant mothers, however, the reduction of $p g c$ FISH staining intensity at the very posterior pole is less pronounced than in wild type embryos (compare Fig. $16 \mathrm{E}$ and $\mathrm{J}$ ). A y-projection of a complete confocal stack of a posterior pole shows clearly shows that phenomenon (compare Fig. $18 \mathrm{~A}^{\prime}$ and $\mathrm{B}^{\prime}$ ), while the distribution of Vasa protein in lost and wild type condition is highly similar (Fig. 18 A" and B").

The less pronounced reduction of $p g c$ FISH staining intensity in the center of the germ plasm is also reflected in the averaged intensity profile (Fig. $18 \mathrm{C}$, red). At the margins of the germ plasm the normalized staining intensity reaches 0.8 which poses no difference between wild type and maternally lost mutant embryos. In the center of the germ plasm, however, the $p g c$ FISH staining intensity is reduced to only 0.6 in lost embryos, compared to a reduction to 0.4 in wild type embryos (Fig. $18 \mathrm{C}$ ). The difference between the averaged curves is highly statistically significant in its center (Mann-Whitney-U test, $\mathrm{p}<0.001$ ) and not significant at the margins of the germ plasm. The analysis of germ plasm RNAs by FISH reveals a uniform distribution of $g c l, c y c B$ and osk RNA within the germ plasm of wild type embryos and a reduced staining intensity in the center of the germ plasm for nos and pgc RNA. This reduction in staining intensity is highly reproducible and can be quantified by averaging intensity 

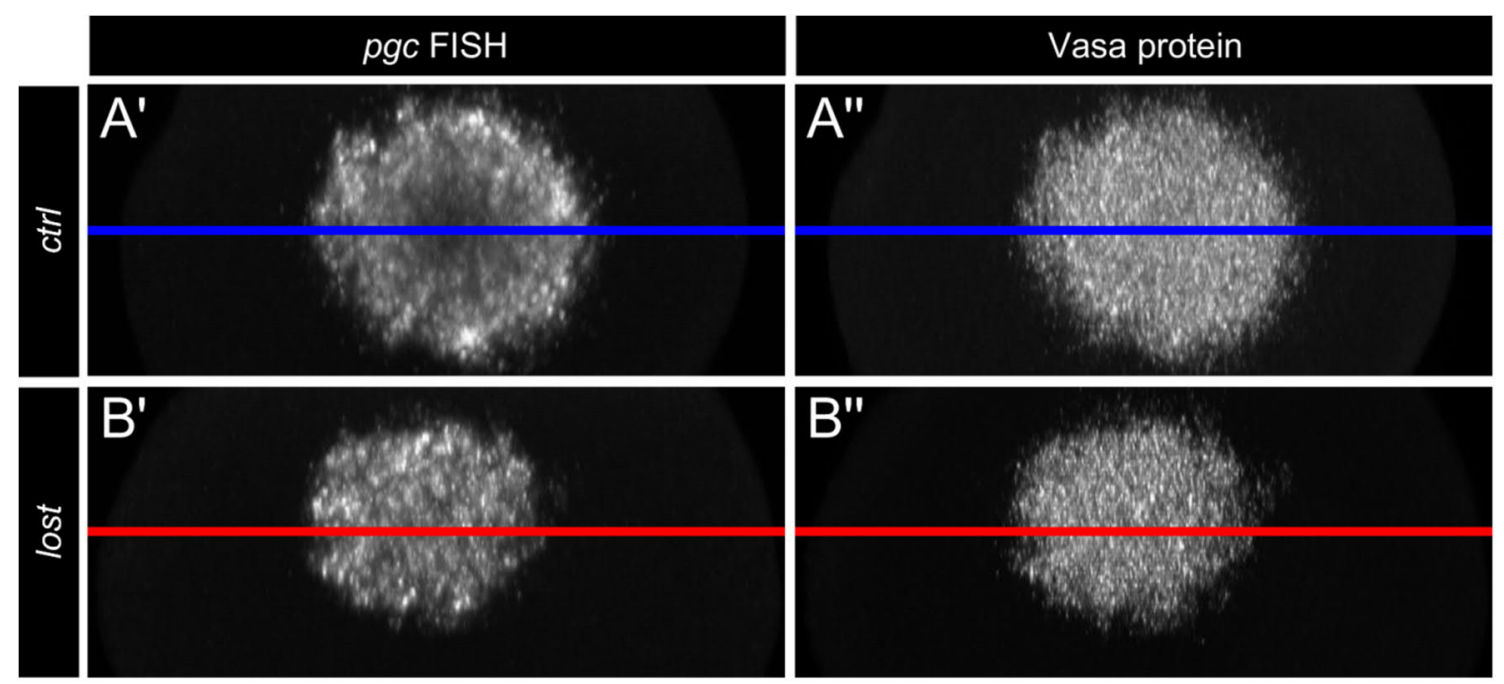

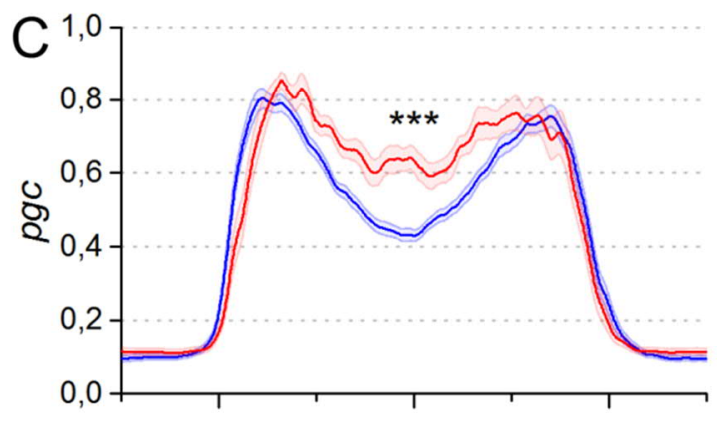

relative germ plasm position

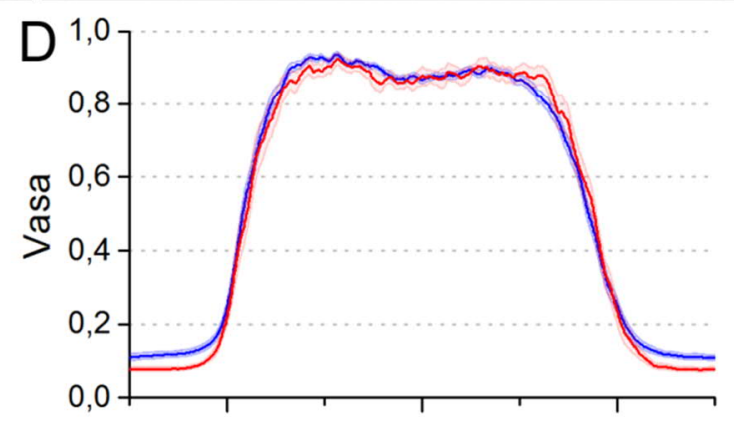

relative germ plasm position
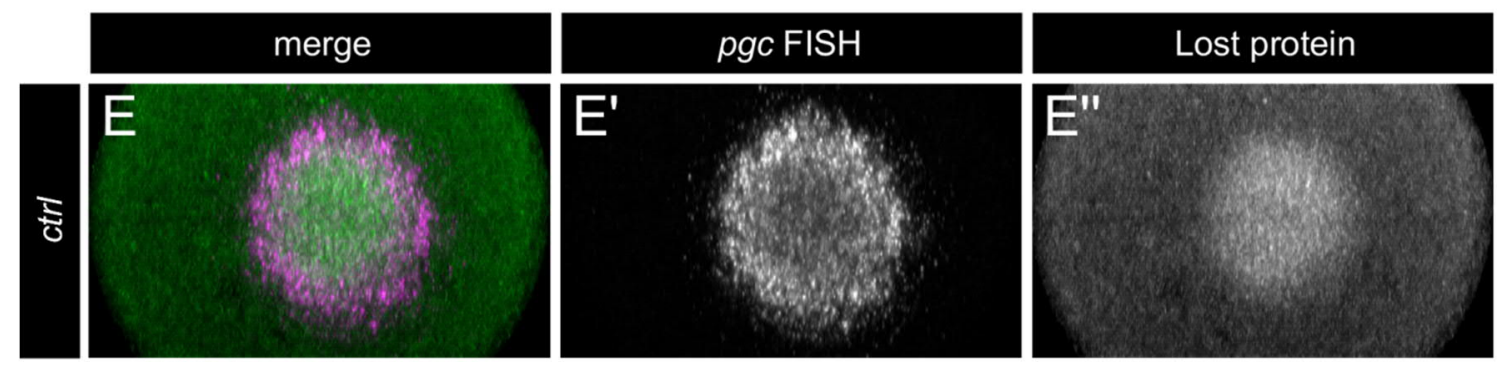

Figure 18: The average relative pgc FISH staining intensity in the center of the germ plasm is higher in maternal lost embryos. $(\mathrm{A}, \mathrm{B}) \mathrm{y}$-projections of confocal stacks covering the complete germ plasm stained for $p g c$ RNA and Vasa protein. (C,D) Average intensity profiles of $p g c$ FISH (C) and the corresponding Vasa channel (D) from control (blue) and maternal lost embryos (red) along a straight line through the center of the germ plasm (see red and blue lines in (A,B)) in y-projections of confocal stacks covering the complete germ plasm stained for $p g c$ RNA and Vasa protein. The shaded areas show the SEM. The intensity profile of $p g c(C)$ shows a depression reflecting the lower $p g c$ FISH staining intensity in the center of the germ plasm. The depression is significantly less deep in maternal lost embryos compared to control (*** $\mathrm{p}<0.001$ Mann-Whitney-U test). (E) y-projections of a confocal stack covering the complete germ plasm of a wild type embryo stained for $p g c$ RNA and Lost protein. Lost protein is enriched in the center of the germ plasm, where the $p g c$ FISH staining intensity is lower. 
profile curves. The quantitative analysis reveals a statistically significant weaker reduction in $p g c$ FISH staining intensity in the center of the germ plasm of lost embryos compared to wild type embryos. No difference was observed for $g c l, c y c B$, osk and nos RNA, as well as for the distribution of Vasa protein.

\section{Maternal lost mutants combine features of $p g c$ LOF and $p g c$ GOF}

Of all analyzed germ plasm RNAs only $p g c$ FISH staining shows a different behavior in lost embryos. pgc (polar granule component) codes for a 71-amino acid peptide that plays a critical role in the transcriptional silencing of the PGCs (Hanyu-Nakamura et al. 2008). Overactivity of $p g c$ results in a "pole-hole" phenotype: Embryos from mothers, who carry 4 additional copies of the genomic $p g c$ locus $(6 x[p g c])$, show cellularization defects at the posterior pole; likely to be caused by a decrease in somatic transcription due ectopic action of Pgc in the somatic nuclei (de Las Heras et al. 2009). That polehole phenotype is strikingly similar to the premature transmigration of PGCs seen in maternally lost mutant embryos (compare Fig. 19 B and C). As described earlier the number of transmigrating PGCs can be used to quantify the strength of the phenotype. In embryos from $6 x[\mathrm{pgc}]$ mothers on average 12 PGCs (40\%) transmigrate, compared to $11(23 \%)$ in maternal lost embryos and 6 (13\%) in wild type embryos (Fig. 19 E). The differences between $6 x[\mathrm{pgc}]$ or lost and wild type, respectively, are statistically significant (Mann-Whitney-U test, $* * \mathrm{p}<0.01$. *** $\mathrm{p}<0.001)$.

If two genes or their products function in the same biological process the combination of respective mutations can result in phenotypes which are much stronger than expected from the individual phenotypes. This phenomenon is called genetic interaction and can be used to identify functional relationships between genes (Mani et al. 2008).

In embryos from mothers heterozygous for lost (lost/+) the absolute as well as the relative number of transmigrating PGCs are not significantly different from wild embryos (Fig. 19 E,F). A very slight increase of $p g c$ activity, in embryos from mothers having just 1 additional copy of the genomic $p g c$ locus $(3 x[p g c])$, does not lead to a pole-hole phenotype, as the absolute and the relative number of transmigrating PGCs differ not significantly from wild embryos (Fig. 19 E,F). However, when combining 

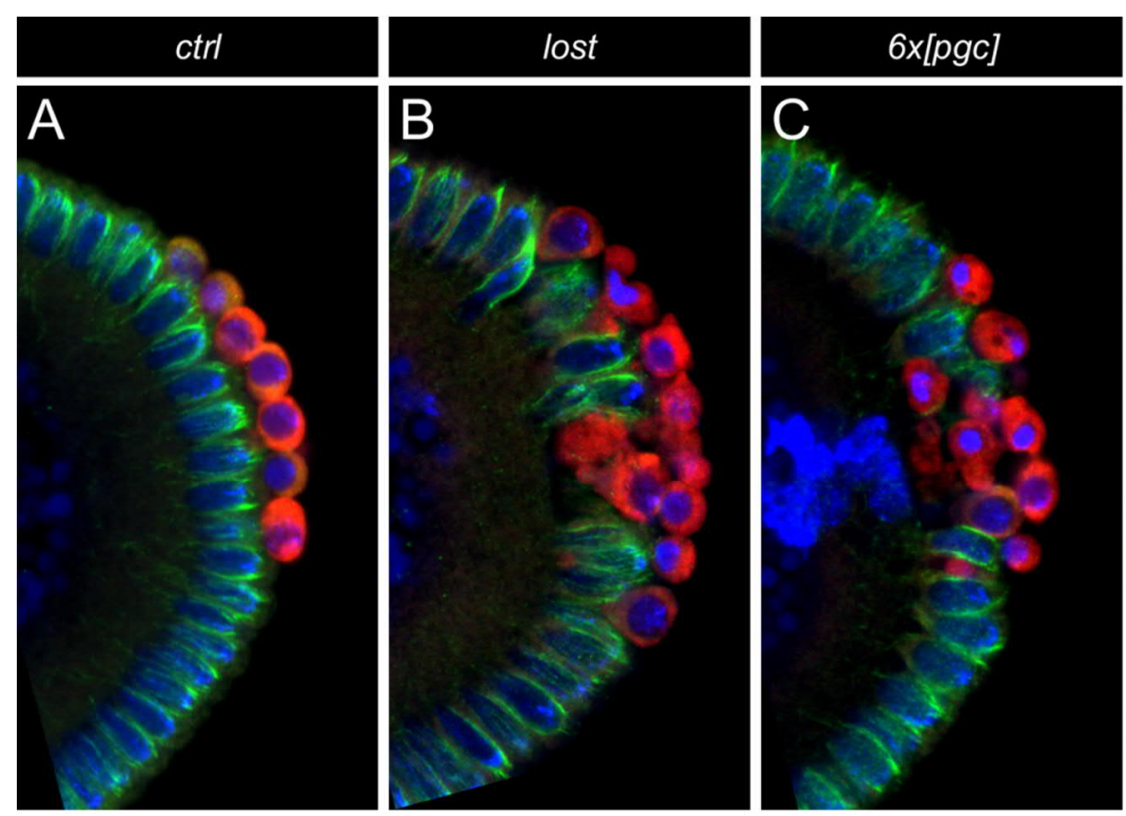

$3 \times[p g c] ; ; / o s t /+$
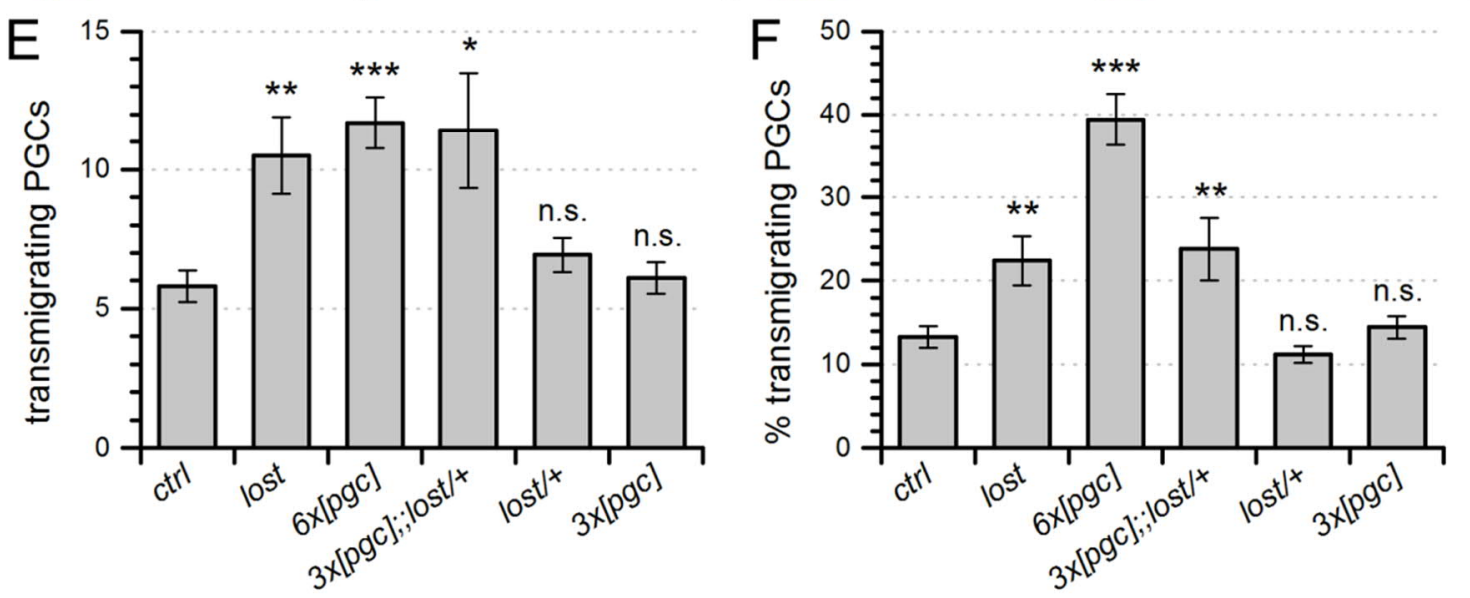

Figure 19: lost interacts genetically with $p g c$ GOF. (A-D) Maximum intensity projections of 2-5 confocal sections of posterior poles of stage 6 embryos stained for Vasa (red), Tubulin (green) and Dapi (blue). (A) In control embryos the PGCs reside at the posterior pole. (B,C,D) In lost embryos, $6 x[p g c]$ embryos and $3 x[p g c] ; ;$ lost/+ embryos some PGCs migrate through the epithelium. (E,F) Quantification of the premature transmigration phenotype. The columns show the average total number (E) or relative number (F) of transmigrating PGCs during stages 6 and 7 of the indicated genotypes. While heterozygous lost embryos and $3 x[\mathrm{pgc}]$ embryos don't differ from control embryos the combination of both $(3 x[\mathrm{pgc}] ; ;$ lost/+ embryos) leads to premature transmigration as strong as in lost embryos. Error bars show SEM. *** $\mathrm{p}<0.001$. ** $\mathrm{p}<0.01$. ${ }^{*} \mathrm{p}<0.05$ Mann-Whitney-U test.

these two conditions, in embryos from $3 x[\mathrm{pgc}] ; ;$ lost/+ mothers, a clear pole-hole phenotype can be observed (Fig. 19 D). A quantification of the phenotype reveals that on average 12 PGCs (24\%) transmigrate which is significantly different from wild type embryos (Fig. 19 E,F. Mann-Whitney-U test, * p<0.05. ** $\mathrm{p}<0.01$ ). An increased maternal load of $p g c$ (in embryos from $6 x[\mathrm{pgc}]$ mothers) leads to a pole-hole phenotype, 
which is highly similar to the lost premature transmigration phenotype. When combining a slight increase in maternal pgc load (3x[pgc]) and heterozygous lost condition, which both don't show a transmigration phenotype on their own, the resulting embryos from $3 x[\mathrm{pgc}] ; ;$ lost/+ mothers show a pole-hole phenotype, which is as strong as in embryos from homozygous lost mutant mothers. This indicates a genetic interaction between lost and $p g c \mathrm{GOF}$.

In wild type embryos the PGCs are transcriptionally silent after they are formed and become transcriptionally active later in embryonic development (Zalokar 1976, Seydoux and Dunn 1997). Pgc is essential for the repression of transcription in the PGCs, as is embryos from $p g c$ mutant mothers the PGCs show ectopic transcriptional activity (Hanyu-Nakamura et al. 2008). As described above (see Fig. 12) in lost embryos the PGCs also become transcriptionally active after they are formed. In order to compare these phenotypes embryos from wild type mothers, from lost mothers and from two different $p g c$ LOF conditions ( $p g c / D f$ mothers and mothers expressing dsRNA against $p g c$ during oogenesis (maternal $p g c$ RNAi)) were analyzed. Fixed stage 6 embryos of these genotypes were stained with an antibody against active RNApolymerase II to show transcription (pCTD), an anti-Vasa antibody to label the PGCs and Dapi to show the nuclei. Then 3D confocal stacks were acquired and the total number of PGCs as well as the number of transcriptionally active PGCs, determined by the presence of a nuclear pCTD signal, was counted.

Figure 20 shows the posterior poles of stage 6 embryos from wild type mothers (A,A'), lost mutant mothers (B, $\left.\mathrm{B}^{\prime}\right)$, pgc/Df mothers $\left(\mathrm{C}, \mathrm{C}^{\prime}\right)$ and mothers with RNAi-mediated pgc knockdown. In wild type embryos around $20 \%$ of the PGCs are transcriptionally active, while in lost embryos almost $60 \%$ of the PGCs show transcriptional activity. In embryos from mothers devoid of $p g c$ function the amount of PGCs which have active transcription is drastically increased to $85 \%$ in embryos from $\mathrm{pgc} / \mathrm{Df}$ mothers and $98 \%$ in embryos from $p g c$ RNAi mothers. These differences are statistically significant compared to wild type numbers (Mann-Whitney-U test, $* * * \mathrm{p}<0.001$ ).

In summary, Embryos from lost mutant mothers and pgc LOF mothers both show the ectopic transcriptional activity in the PGCs, although the phenotype is stronger in $p g c$ LOF condition. 

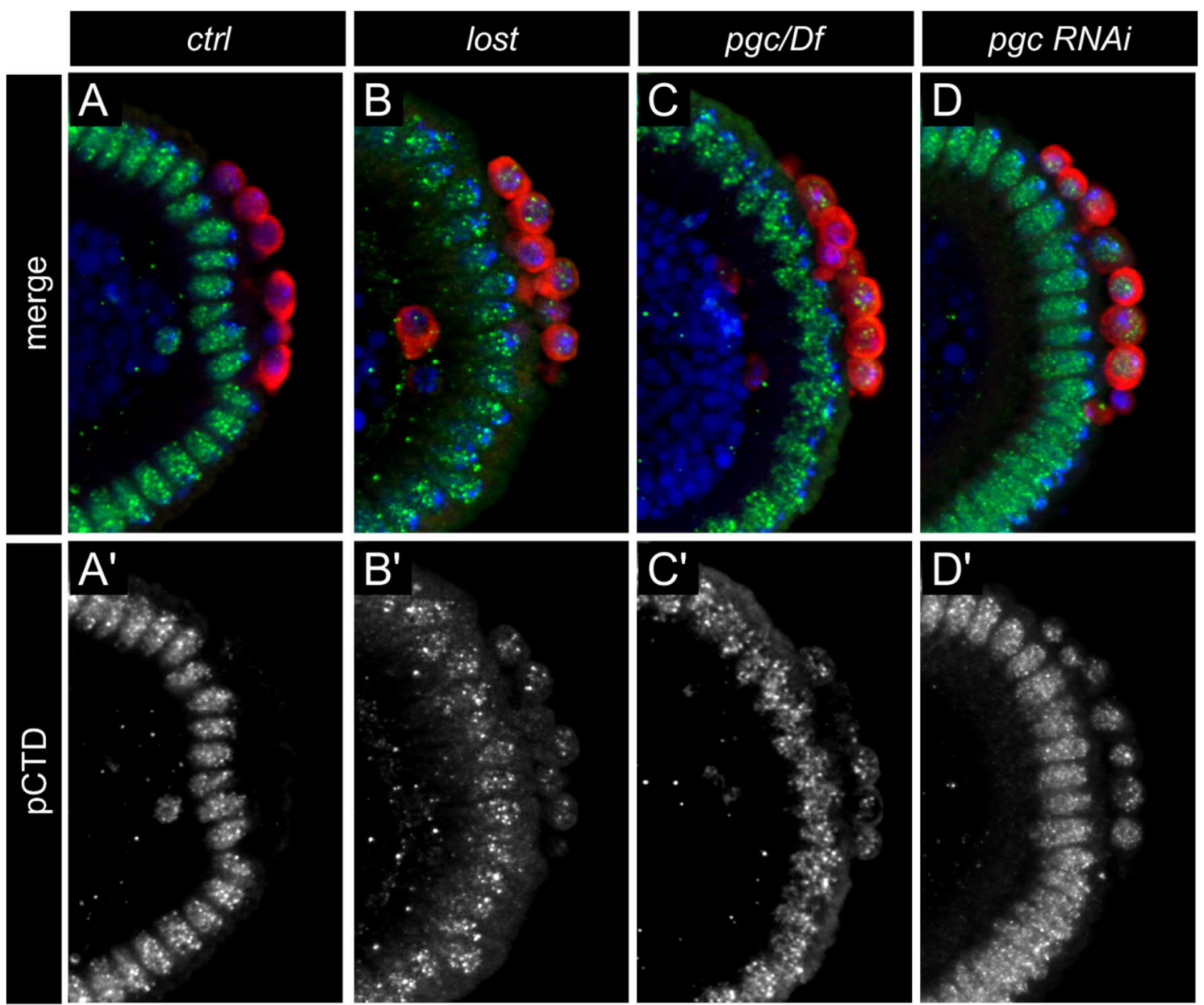

Figure 20: The PGCs in embryos which lack maternal lost or maternal pgc show ectopic transcriptional activity. (A-D) Maximum intensity projections of 2-5 confocal sections of posterior poles of stage 6 embryos stained for Vasa (red), pCTD (green) and Dapi (blue). The presence of a pCTD signal indicates transcriptional activity. (A) In wild type embryos only the somatic cells but not the PGCs show a strong pCDT signal (A'). (B-D) In embryos

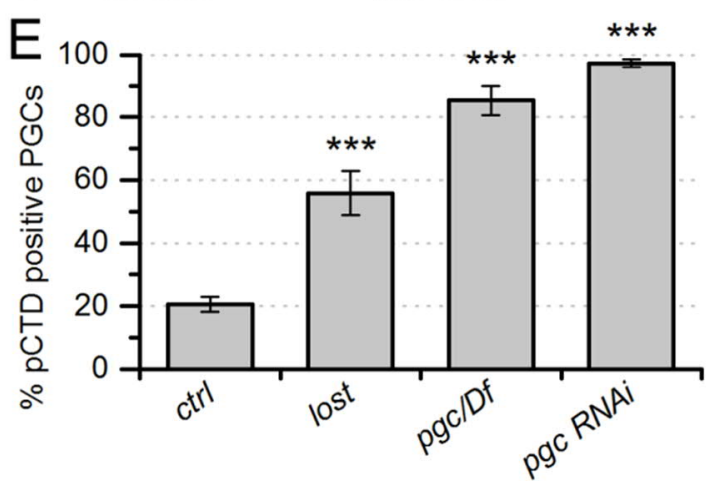
which lack the maternal lost contribution (A) or maternal pgc (C,D) many PGCs show a clear pCTD signal (B'-D'). (E) Quantification of the ectopic transcriptional activity of the PGCs. The columns show the average relative number of pCTD-positive PGCs in stage 6 embryos of the four genotypes. Error bars show SEM. *** $p<0.001$. ** $p<0.01$ Mann-Whitney-U test. 


\section{$\underline{\text { lost interacts genetically with } d h c 64 c}$}

Maternally lost mutant embryos show features of the pgc GOF phenotype in the soma beneath the PGCs (reduced transcriptional activity in the SNUPs and cellularization defects) as well as features of the pgc LOF phenotype within the PGCs (ectopic transcriptional activity). During stage 3 of embryonic development the germ plasm and with it $p g c$ RNA is transported into the forming PGCs via dynein-mediated transport on the astral microtubules (Lerit and Gavis 2011). A reduced efficiency or slower transport could result in an increased amount of $p g c$ RNA in the soma after PGC budding and a reduced amount of $p g c$ RNA within the PGCs. The dynactin complex contains, among others, Actin-related protein 1 (Arp1) and Dynein heavy chain (Dhc) and links different cargoes to microtubule dependent motor proteins (Allan 1996). It has been shown that one Dhc variant, Dhc64c, plays a role in the transport of the germ plasm (Lerit and Gavis 2011). Furthermore, a genome wide yeast two-hybrid screen identified Lost as a possible binding partner of Arp1 (Giot et al. 2003). This interaction was verified in early Drosophila embryos by GST-pulldown and co-immunoprecipitation experiments (Graf 2007). In order to establish a functional relationship between Lost and the dynactin transport complex a genetic interaction experiment was performed. Embryos
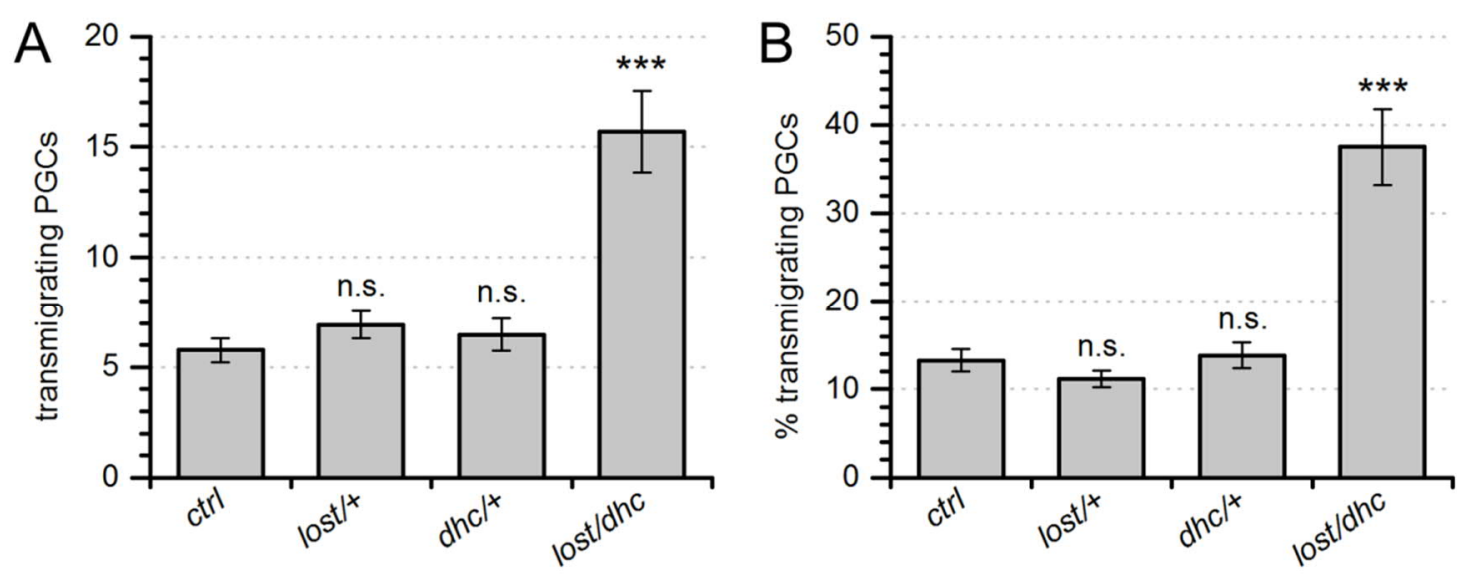

Figure 21: lost interacts genetically with dynein heavy chain $(d h c)$. (A,B) Quantification of the premature transmigration phenotype. The columns show the average total number (A) or relative number (B) of transmigrating PGCs during stages 6 and 7 of the indicated genotypes. While heterozygous lost embryos and heterozygous $d h c$ embryos don't differ from control embryos the combination of both (lost/dhc embryos) leads to a strong premature transmigration phenotype. Error bars show SEM. *** $\mathrm{p}<0.001$ Mann-Whitney-U test. 
from wild type mothers ( $\mathrm{ctrl}$ ), heterozygous lost mutant mothers (lost/+), heterozygous dhc64c mutant mothers ( $d h c /+)$ and from mothers transheterozygous for lost and dhc64c (lost/dhc) were fixed and stained with an antibody against Vasa to label the PGCs. The number of transmigrating PGCs was used to quantify the phenotype.

Figure 21 shows the average absolute (Fig. 21 A) and relative (Fig. 21 B) numbers of transmigrating PGCs in embryos of the before mentioned genotypes. In wild type embryos around 6 PGCs (14\%) transmigrate. In embryos from mothers heterozygous for lost (lost/+) on average 7 PGCs (12\%) transmigrate and in embryos from mothers heterozygous for $d h c 64 c(d h c /+)$ on average $6.5(14 \%)$. These numbers are not significantly different from wild type embryos. However, when combining these two conditions, in embryos from lost/dhc mothers, the amount of transmigrating PGCs is 16 $(38 \%)$. This increase is statistically significant when compared to wild type (MannWhitney-U test, *** $\mathrm{p}<0.001)$. This indicates a genetic interaction between lost and dhc64c.

\section{$\underline{\text { No detectable change in } p g c \text { RNA or peptide localization in maternal lost }}$} $\underline{\text { mutants }}$

Lost protein was shown to be a part of different RNP complexes (Herold et al. 2009, Snee and Macdonald 2009) and is enriched at the posterior pole of very early embryos (Fig. 18 E"). Furthermore, lost interacts biochemically (Graf 2007) and genetically (Fig. 21) with components of the dynactin transport complex which has been shown to have a function in germ plasm transport (Lerit and Gavis 2011). This raises the possibility that Lost acts as an adapter protein linking germ plasm RNPs to the dynactin complex and by that promotes the transport of $p g c$ RNA into the budding PGCs. If Lost facilitates the transport of $p g c$ RNA into the PGCs an increased amount of $p g c$ RNA should be detectable in the soma after PGC budding in embryos from lost mutant mothers. In order to test this hypothesis fixed embryos from wild type or lost mutant mothers were analyzed. $p g c$ RNA was visualized using FISH, an antibody against Vasa and Dapi were used to label the PGCs and the nuclei, respectively. Figure 22 shows the posterior poles 


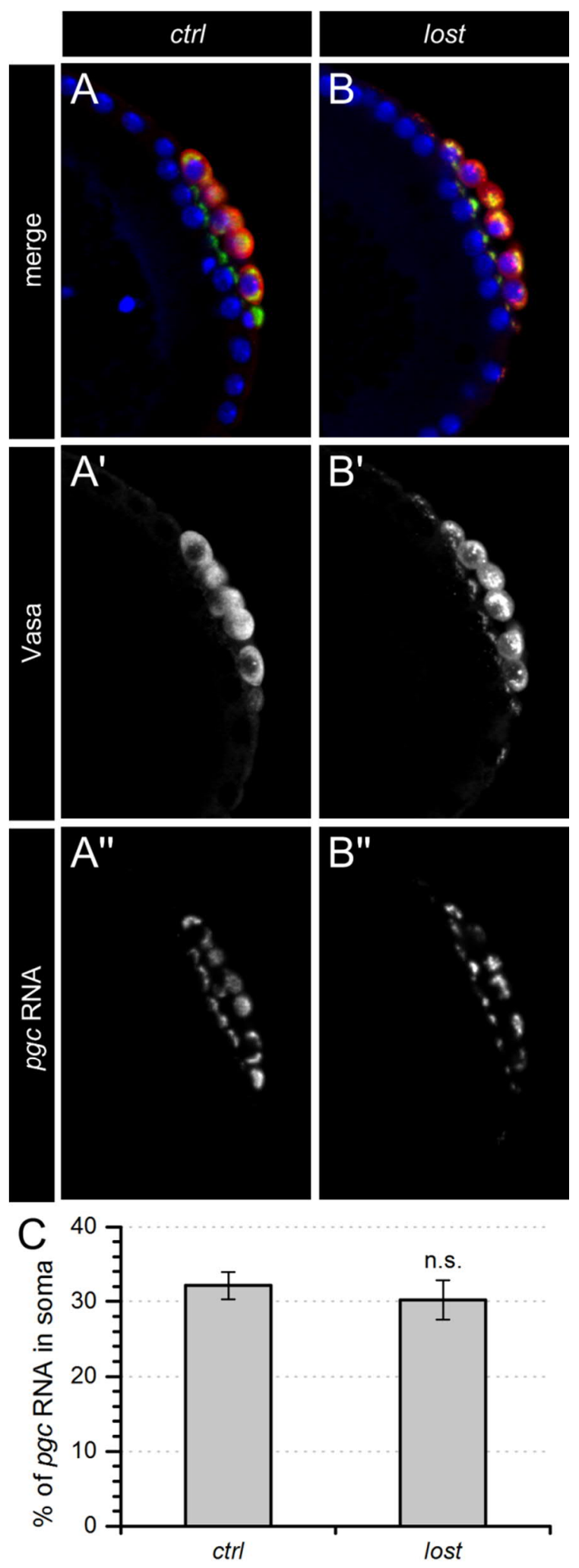

Figure 22: The amount of $p g c$ RNA which remains in the soma after PGC formation is not increased in maternal lost mutants. (A,B) Maximum intensity projections of 2-4 confocal sections of posterior poles of stage 4 embryos stained for Vasa (red), $p g c$ RNA (green) and Dapi (blue). $\left(A^{\prime}, B^{\prime}\right)$ Vasa staining clearly labels the PGCs. $(\mathrm{A} " \mathrm{~B} ")$ A considerable portion of the $p g c$ RNA can be detected in the soma after PGC formation in both wild type and lost mutant embryos, located apical of the SNUPs. (C) Quantifaction of the $p g c$ FISH signal in the soma. The columns show the average cumulative $p g c$ FISH signal intensity located in the soma relative to the cumulative total $p g c$ FISH signal intensity of a given embryo. The relative amount of $p g c$ FISH signal in the soma in maternal lost embryos does not differ significantly from wild type embryos. Error bars show SEM. n.s. not significant, MannWhitney-U test.

of a wild type embryo (Fig. 22 A) and a lost embryo (Fig. 22 B). After PGC budding is completed the PGCs reside at the posterior pole separated from the soma (Fig. $22 \mathrm{~A}^{\prime}, \mathrm{B}^{\prime}$ ). In both genotypes $p g c$ RNA is detected within the PGCs as well as in the soma adjacent to the PGCs (Fig. 22 $\mathrm{A} " \mathrm{~B} ")$. There is no obvious difference in the amount of somatic pgc RNA between wild type and lost embryos. In order to quantify the distribution of $p g c$ RNA within an embryo a Fiji macro was created for batch processing each embryo individually (see Methods for details). 
In brief, 3D confocal stacks covering the complete posterior pole were analyzed. Because of some Vasa protein left in the soma after PGC budding it is not possible to use a mask from the Vasa channel to distinguish between somatic $p g c$ RNA and $p g c$ RNA which has been transported into the PGCs. Instead the identity of the nuclei (somatic or PGC nucleus) can be used since the somatic pgc RNA always localizes to an area directly apical of the somatic nuclei underneath the PGCs (Fig. 22 A",B"). For that in the Dapi channel each nucleus was identified as a single object using a 3D watershed algorithm. Then each nucleus was assigned to be either somatic or within a PGC by measuring the average intensity of Vasa signal in a sphere around that nucleus. Then the $p g c$ FISH signal in a volume around all somatic nuclei was measured and compared to the $p g c$ FISH signal which is not in that volume (i.e. the $p g c$ FISH signal within the PGCs). This yields the relative amount of pgc RNA which remains in the soma after PGC formation is complete for each individual embryo. On average $32 \%$ of the pgc FISH signal is located in soma in wild type stage 4 embryos and $30 \%$ in embryos from lost mutant mothers (Fig. 22 C). The difference is not statistically significant (Mann-Whitney-U test, $\mathrm{p}=0.71$ ). There is no detectable difference in the amount of $p g c$ RNA which remains in the soma after PGC budding between wild type and lost embryos.

The transcriptional silencing of the SNUPs which is seen in lost embryos could be explained by a temporal or spatial misregulation of $p g c$ translation, although no change in $p g c$ RNA localization could be detected after PGC formation in lost embryos compared to wild type embryos (Fig. 22). pgc RNA codes for a 71-amino acid peptide which globally blocks transcriptional elongation (Hanyu-Nakamura et al. 2008). In order to visualize the Pgc peptide fixed stage 4 embryos from either wild type or lost mutant mothers were stained with an antibody against Pgc, an antibody against Vasa and with Dapi (Fig. 23 A,C). As reported earlier (Hanyu-Nakamura et al. 2008) in wild type embryos Pgc is enriched in the nuclei of the PGCs and in their cytoplasm but it is absent from the somatic portion of the embryo (Fig. 23 A"). The same localization pattern is found in lost embryos (Fig. 23 C"). In both genotypes no Pgc signal could be detected in the soma beneath the PGCs which stands out from the background noise. Another way of visualizing the translation of $p g c$ RNA is to use a transgenic reporter 


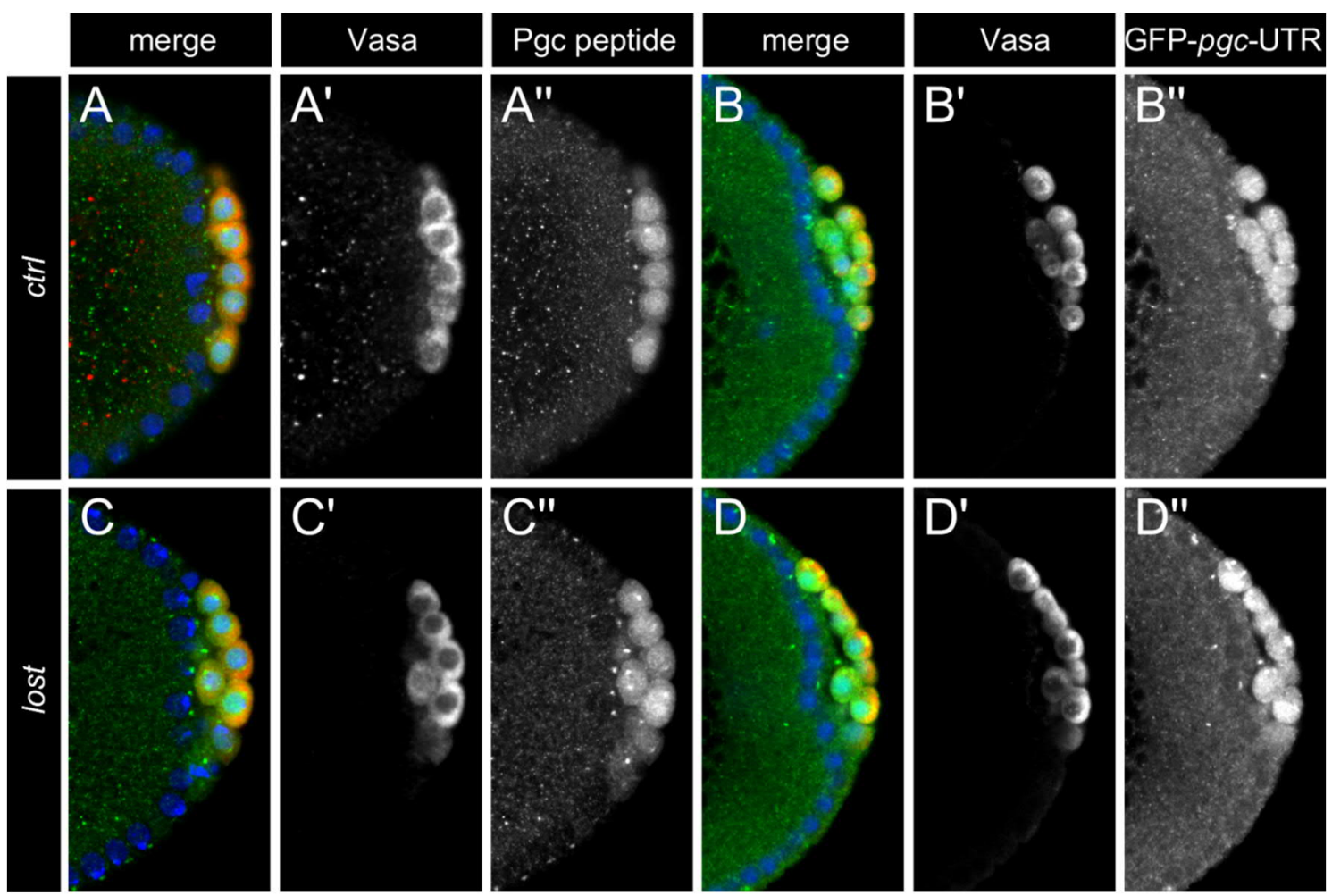

Figure 23: Maternal loss of lost does not drastically affect the localization of Pgc peptide or a $p g c$ translation reporter construct after PGC formation. (A-D) Maximum intensity projections of 2-4 confocal sections of posterior poles of stage 4 embryos stained for Vasa (red), Pgc peptide or GFP reporter (green) and Dapi (blue). (A'-D') Vasa staining clearly labels the PGCs. (A",B") Pgc peptide is readily detected in the PGCs (enriched in the nuclei) but not in the soma after PGC formation with no obvious difference between wild type and lost embryos. (B") The translation of GFP from a maternally provided GFP-pgc-3'-UTR transgene recapitulates the translation of $p g c$ (Rangan et al. 2009). GFP is detected in the PGCs but not in the soma. (D") Maternal loss of lost does not affect the expression of the GFP reporter. The low signal-to-noise ratio in (A"-D") does not allow to exclude subtle effects.

construct described by Rangan et al. (2009). It has been shown that the 3'-UTR of a given mRNA dictates its subcellular localization and translational regulation (Rangan et al. 2009). A transgene consisting of the open reading frame (ORF) of eGFP fused to the pgc-3'-UTR under the control of the maternally active nos promotor can be used to highlight the spatial and temporal regulation of the translation of pgc RNA (Rangan et al. 2009). Fixed stage 4 embryos from mothers homozygous for the above mentioned transgenic reporter construct either in wild type genetic background or lost mutant background have been stained with antibodies against GFP and Vasa and with Dapi (Fig. $23 \mathrm{~B}, \mathrm{D})$. In control embryos the GFP reporter is highly enriched in the PGCs, as already published (Rangan et al. 2009). No signal which is stronger than the background noise 
can be detected in the soma beneath the PGCs (Fig. 23 B"). This pattern reflects the translation of $p g c$ RNA in wild type embryos. In embryos from lost mutant mothers which also express the transgenic reporter construct the pattern of the GFP reporter is indistinguishable from the pattern in control embryos: The GFP reporter can be detected in the PGCs, but not in the adjacent soma (Fig. 23 D").

There is no obvious difference in the translation of $p g c$ RNA between wild type embryos and lost embryos as judged by Pgc antibody staining and a transgenic reporter construct which recapitulates $p g c$ translation. However, due to the high levels of background noise in the stainings a weak somatic signal of $\mathrm{Pgc}$ or the GFP reporter cannot be excluded.

\section{An excess of the $p g_{c}$ 3'UTR causes lost-like phenotypes}

Fixed embryos from mothers overexpressing the GFP-pgc-3'UTR transgenic construct described above in a wild type background were stained with an anti-Vasa antibody to label the PGCs, an anti-pSerCTD antibody to show transcriptional activity and Dapi to label the nuclei. Figure 24 A shows the posterior pole of a stage 6 embryo. The embryo shows a pole-hole phenotype with cellularization defects at the posterior pole and transmigrating PGCs (Fig. 24 A'). This is highly similar to lost embryos (see Fig. 12) and to maternal pgc overexpression (Fig. 19). Quantification of that phenotype by calculating the relative amount of transmigrating PGCs reveals that the relative number of transmigrating PGCs is increased to $21 \%$ while in wild type embryos only $14 \%$ of the PGCs transmigrate (Fig. $24 \mathrm{~B}$ ). This increase compared to wild type embryos is statistically significant (Mann-Whitney-U test, * ${ }^{*}<0.05$. ** $\mathrm{p}<0.01$ ). The amount of transmigrating PGCs in maternal lost mutants is shown for comparison (see Fig. 12 for details).

Furthermore, an ectopic transcriptional activity can be detected in the PGCs in stage 6 embryos from mothers overexpressing GFP-pgc-3'UTR as evident by the nuclear pCTD staining in the PGCs (Fig. 24 A,A"). The same phenotype can be found in lost embryos (Fig. 13) and in maternal pgc mutant embryos (Fig. 20). Quantification of that phenotype by calculating the relative amount of transcriptionally active PGCs reveals 
Figure 24: Embryos from mothers expressing GFPpgc-3'UTR show lost-like phenotypes. (A) Maximum intensity projections of 4 confocal sections of posterior poles of stage 6 embryos stained for Vasa (red), pCTD (green) and Dapi (blue). The presence of a pCTD signal indicates transcriptional activity. (A') Some of the PGCs, labeled by Vasa, migrate through the epithelium prematurely. (A") Some PGCs show ectopic transcriptional activity. (B) Quantification of the premature transmigration and (C) transcriptional active PGCs in GFP-pgc-UTR
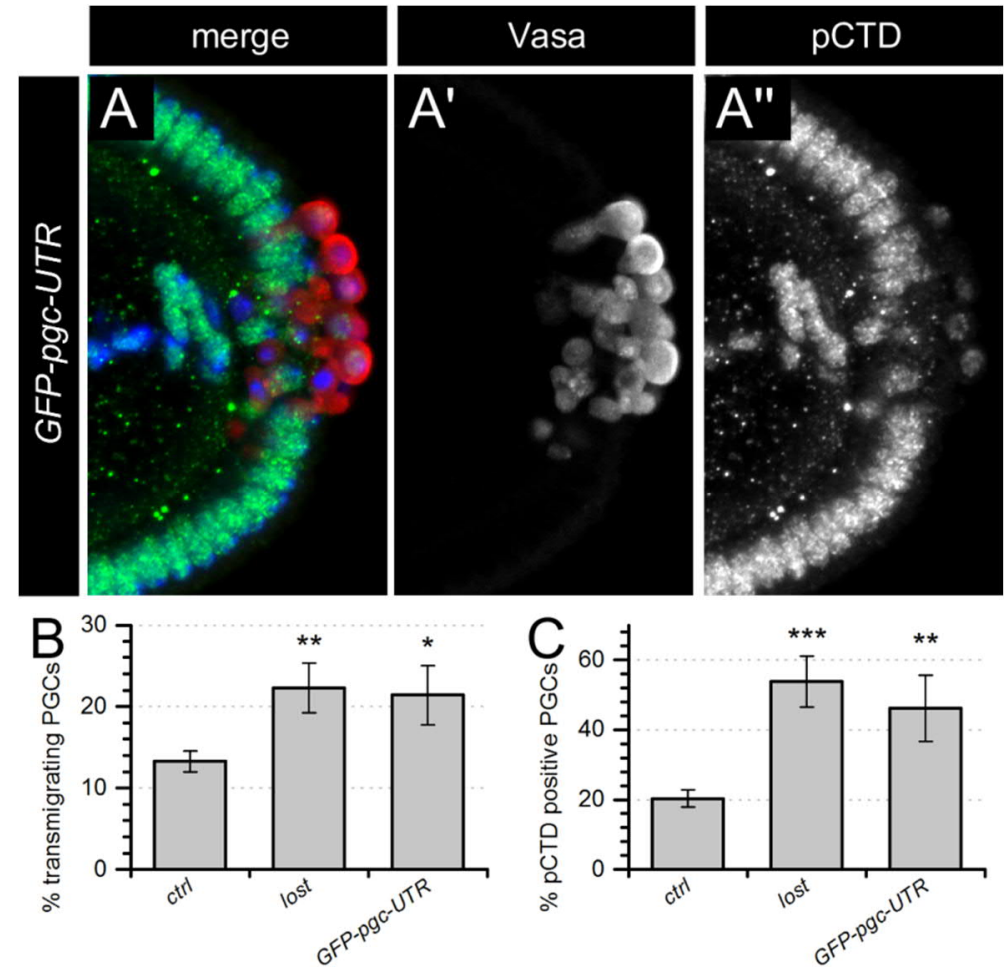

embryos in comparison wild type ( $\mathrm{ctrl}$ ) and maternal lost embryos. The columns show the average relative number of transmigrating PGCs during stages 6 and 7 (B) or pCTD positive PGCs during stage 6 (C) of the indicated genotypes. The relative amount of transmigrating PGCs and transcriptionally active PGCs in GFP-pgc-UTR embryos is significantly increased compared to wild type embryos, but does not differ significantly from maternal lost embryos. Error bars show SEM. *** $\mathrm{p}<0.001$. ** $\mathrm{p}<0.01$. $* \mathrm{p}<0.05$ Mann-Whitney-U test.

that the relative number of transcriptionally active PGCs is increased to $48 \%$ while in wild type embryos only $20 \%$ of the PGCs have active transcription (Fig. 24 C). This increase compared to wild type embryos is statistically significant (Mann-Whitney-U test, $* * p<0.01$. $* * * p<0.001)$. The amount of transcriptionally active PGCs in maternal lost mutants is shown for comparison (see Fig. 13 for details).

Embryos from mothers which overexpress GFP-pgc-3'UTR exhibit features from maternal pgc mutants (ectopic transcriptional activity in the PGCs) and from $p g c$ GOF embryos (transmigrating PGCs). This combination of phenotypes is also seen in embryos from lost mutant mothers. 
The amount of osk RNA in the PGCs is increased in maternal lost mutants

These phenotypes are not caused by an increased amount of the complete pgc RNA in the early embryo but only its 3'-UTR. The 3'-UTR of a given RNA dictates its localization, stability and translation by binding to certain proteins forming ribonucleoprotein particles (RNPs) (reviewed by Martin and Ephrussi (2009)).

These RNPs can consist of different proteins and RNAs which define their function and subcellular localization. The RNPs which contain the germ line determinants are referred to as "polar granules" and are located at the posterior pole in the early embryo. Since Lost protein has been found to be present in various RNPs (Herold et al. 2009, Snee and Macdonald 2009) and it is enriched at the posterior pole (Fig. 11 C) it might play a role in regulating the germ plasm RNPs.

Recently it was found that the pole plasm contains at least two different types of RNPs (Trcek et al. 2015). One type, the polar granules, consists of Vasa and Osk proteins and $n o s, g c l, c y c B$ and $p g c$ RNAs. $p g c$ RNA, however, is the only germ plasm RNA which is also found in the other type of RNP (Trcek et al. 2015). Besides pgc RNA and Vasa protein this RNP also contains osk RNA, which is absent from the polar granules (Trcek et al. 2015). In contrast to the polar granule RNAs osk RNA is excluded from PGCs (Little et al. 2015) although it inhabits the same volume at the posterior pole of an embryo as the germ plasm.

Since $o s k$ and $p g c$ are found in the same RNP and loss of lost has an effect on pgc FISH intensity profile (see Fig. 18) a possible effect of Lost on the localization of $o s k$ after PGC formation was investigated. For that wild type and maternal lost mutant embryos have been stained and analyzed. Figure 25 shows the posterior poles of fixed stage 4 embryos from either wild type mothers (Fig. 25 A) or lost mutant mothers (Fig. 25 B). osk RNA was visualized using FISH, an anti-Vasa antibody was used to label the PGCs and Dapi to show the nuclei. As published before (Little et al. 2015), the vast majority of osk RNA is found in the soma beneath the PGCs but not in the PGCs in wild type embryos (Fig. 25 A'). The somatic osk RNA is not localized apically or perinuclear, it rather seems to diffuse away from the posterior pole. In lost embryos, however, a substantial fraction of osk RNA signal is found within the PGCs (Fig. 25 B',B"). In order to quantify the relative amount of osk RNA within the PGCs of a stage 4 embryo a 


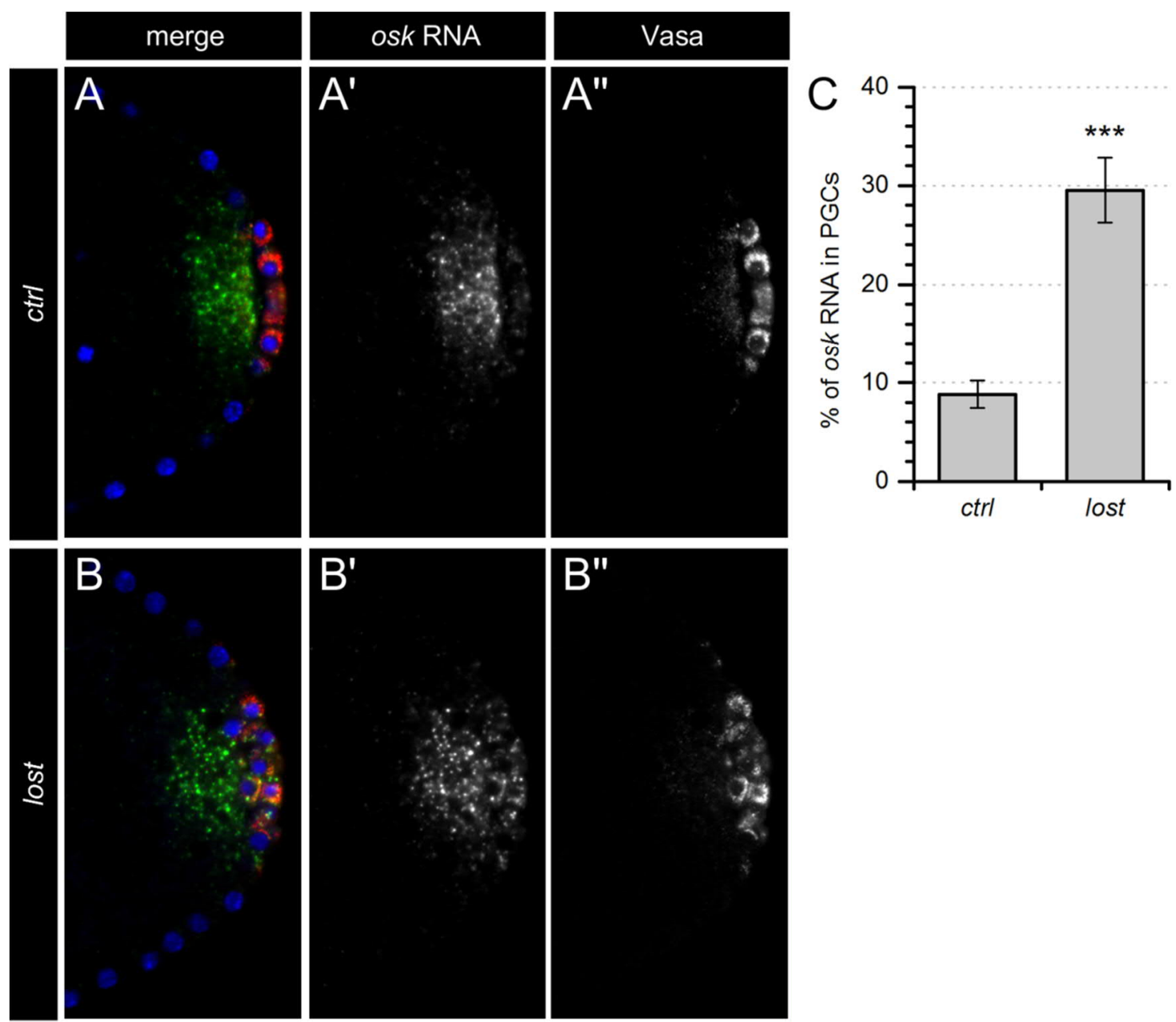

Figure 25: The amount of osk RNA within the PGCs is drastically increased in maternal lost mutants. (A,B) Maximum intensity projections of 2-4 confocal sections of posterior poles of stage 4 embryos stained for Vasa (red), osk RNA (green) and Dapi (blue). (A) In control embryos the vast majority of osk RNA is found in the soma after PGC formation. Only very weak osk FISH signals (A') can be detected in the PGCs, labeled by Vasa (A"). (B) In maternal lost embryos a strong osk FISH signal (B') can be found colocalizing with Vasa (B"). (C) Quantification of the osk FISH signal in the PGCs. The columns show the average cumulative osk FISH signal intensity located in the PGCs relative to the cumulative total osk FISH signal intensity of a given embryo. The relative amount of osk FISH signal in the PGCs in maternal lost embryos is significantly increased compared to wild type. Error bars show SEM. *** $\mathrm{p}<0.001$ Mann-Whitney-U test.

Fiji macro was created for batch processing each embryo individually (see Methods for details). In brief, 3D confocal stacks covering the complete posterior pole were analyzed. Because of some Vasa protein left in the soma after PGC budding it is not possible to use a mask from the Vasa channel to distinguish between soma and PGCs. Instead the identity of the nuclei (somatic or PGC nucleus) can be used. For that each nucleus was identified as a single object using a 3D watershed algorithm. Then each 
nucleus was assigned to be either somatic or within a PGC by measuring the average intensity of Vasa signal in a sphere around that nucleus. Then a mask from the Vasa channel was created from which a volume around the somatic nuclei was removed resulting in a mask specific for the PGCs. Then the osk FISH signal intensity within that mask and outside that mask was measured. This yields the amount of osk RNA within the PGCs relative to the total amount of osk RNA for each embryo. The quantitative analysis reveals that in wild type embryos on average around $9 \%$ of the osk RNA signal is localized within the PGCs while in embryos from lost mutant mothers $40 \%$ of the osk RNA resides inside the PGCs (Fig. 25 C). This difference is statistically significant (Mann-Whitney-U test, $* * * \mathrm{p}<0.001$ ).

This shows that the relative amount of osk RNA in the PGCs of lost embryos is increased fourfold compared to wild type embryos. The biological relevance of excluding osk RNA from the PGCs remains unclear, however, it was reported that forcing osk RNA into the PGCs has a detrimental effect on their development (Little et al. 2015). The details of this phenomenon remain to be explored.

The localizion of osk RNA and protein is highly dynamic during PGC formation

In order to gain further understanding of the behavior of osk RNA during PGC formation and to investigate the effect of the observed osk RNA mislocalization in lost embryos (see Fig. 25) the changes in osk RNA localization before, during and after PGC formation were analyzed in detail. A transgenic construct, in which the localizing elements of the osk-3'-UTR were replaced with the nos 3'UTR, was obtained (Little et al. 2015). In embryos from mothers overexpressing this construct using the UAS-Gal4 system (thereafter referred to as osk-nos-3'UTR embryos) the transgenic osk RNA is ectopically loaded into the PGCs due to the presence of the nos-3'-UTR which dictates its localization (Van Doren et al. 1998b, Little et al. 2015).

In order to evaluate the performance of the ectopic transport of osk RNA into the PGCs and to document the localization of osk RNA during early embryogenesis FISH was 
used to label osk RNA. The germ plasm was labeled using an antibody against Vasa, Dapi was used to detect the nuclei. The antisense RNA probe directed against osk does not discriminate between endogenous and transgenic osk RNA species. Figure 26 shows confocal sections of posterior poles of embryos from wild type mothers (Fig. 26 A,D,G), lost mutant mothers (Fig. 26 B,E,H) and mothers overexpressing osk-nos-3'UTR (Fig. 26 C,F,I) before PGC budding (Fig. 26 A-C), during PGC budding (Fig. 26 D-E) and after PGC budding (Fig. 26 G-I).

Before PGC budding the osk RNA inhabits the same volume as the germ plasm at the posterior pole as indicated by the almost perfect overlap of the osk FISH staining (Fig. $26 \mathrm{~A}^{\prime}-\mathrm{C}^{\prime}$ ) with the anti-Vasa staining (Fig. 26 A"-C"). A close observation reveals that although osk RNA particles and Vasa particles are localized in the same region they are separate structures. This has been reported earlier (Trcek et al. 2015). There is no difference in the localization of $o s k$ RNA between wild type embryos, lost embryos and osk-nos-3'UTR embryos before the formation of the PGCs.

During PGC budding the germ plasm accumulates around the budding nuclei and eventually gets incorporated into the PGCs. This process can be observed in wild type embryos (Fig. 26 D"), lost embryos (Fig. 26 E") and osk-nos-3'UTR embryos (Fig. 26 F") with no apparent difference between these genotypes. osk RNA follows a different pattern of localization. In wild type embryos osk RNA does not accumulate around the budding nuclei and is thus not actively transported into the forming PGCs. Instead the osk RNA starts to diffuse away from the posterior cortex towards the yolk (Fig. 26 D'). These observations confirm the results from Little et al. (2015). In lost embryos, however, an increased amount of osk RNA seems to be present in the polar buds (Fig. 26 E') compared to wild type embryos. The majority of osk RNA diffuses from the cortex into the embryo comparable to wild type embryos. In osk-nos-3'UTR embryos a substantial amount of osk RNA accumulates around the budding nuclei like the germ plasm does (Fig. $26 \mathrm{~F}^{\prime}$ ) while the remaining osk RNA is diffusing into the embryo. The osk RNA particles which follow the germ plasm are likely to be the transgenic osk-nos3'UTR molecules while the diffusing osk FISH signal represents the endogenous osk RNA.

After PGC budding the germ plasm has been almost completely transported into PGCs. There are only small amounts of Vasa particles left in the soma localized directly apical 


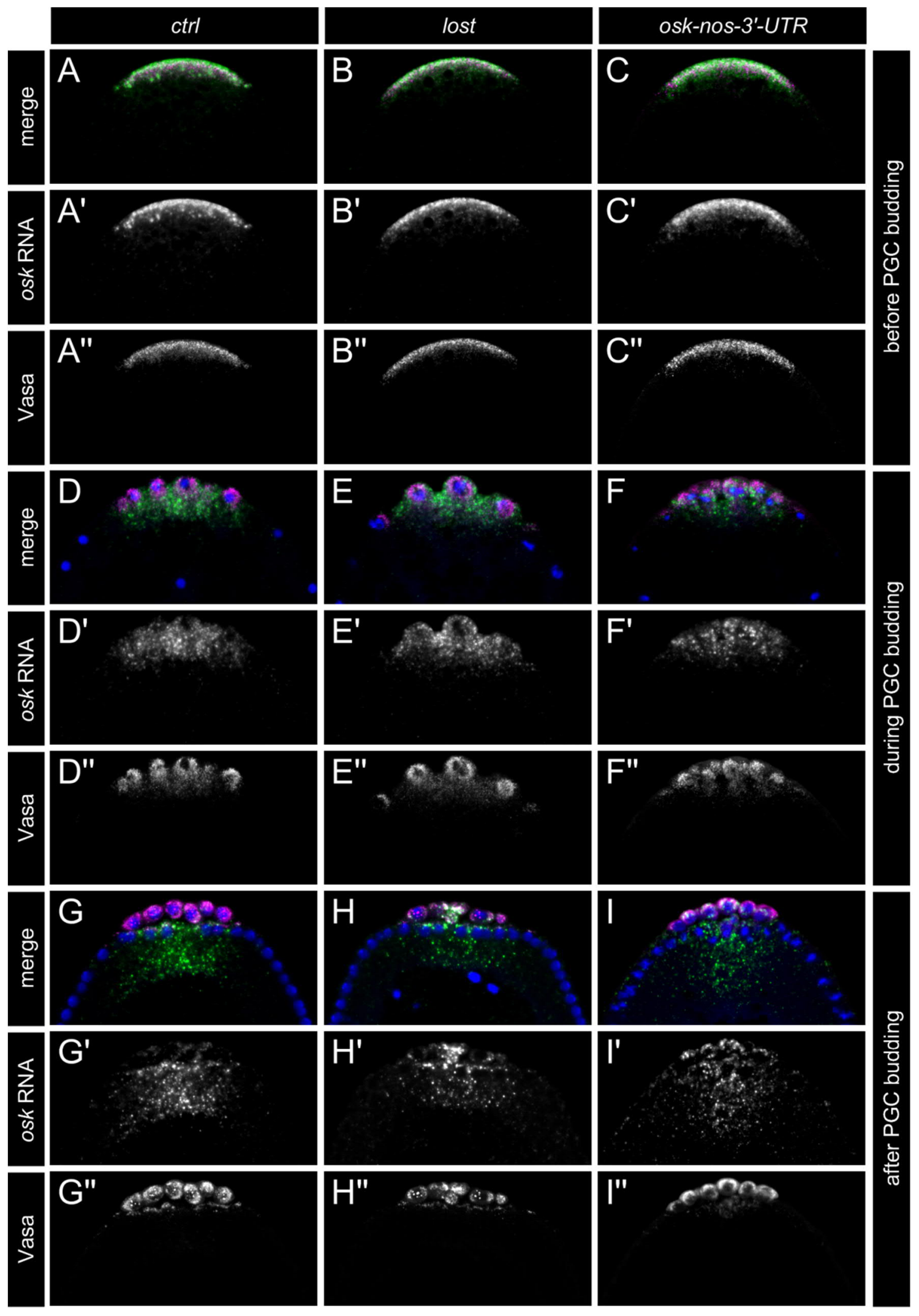


Figure 26: The localization of osk RNA is highly dynamic during PGC formation. (A-I) Maximum intensity projections of 2-4 confocal sections of posterior poles of embryos before (A-C), during (D-F) and after PGC budding (G-I) stained for Vasa (magenta), osk RNA (green) and Dapi (blue). Posterior is up. Note that the osk FISH in $\mathrm{C}^{\prime}, \mathrm{F}^{\prime}$ and I' labels both the endogenous and the transgenic osk RNA species. (A-C) Before PGC budding osk RNA ( $\left.\mathrm{A}^{\prime}-\mathrm{C}^{\prime}\right)$ inhabits the same volume at the posterior pole as the germ plasm labeled by Vasa (A"-C") in wild type, maternal lost mutant embryos and embryos maternally expressing a transgenic RNA composed of the osk ORF and the 3'UTR of nos. (D-F) During PGC budding the germ plasm accumulates around the budding nuclei and is transported into PGCs ( $\left.\mathrm{D}^{\prime \prime}-\mathrm{F}^{\prime \prime}\right)$ with no difference between the genotypes. (D',E') In wild type and maternal lost embryos osk RNA is accumulating around the budding nuclei. Some fraction of osk RNA appears to be brought into the PGCs passively with the posterior cytoplasm. (F') In osk-nos$3^{\prime} U T R$ embryos a large portion of the osk RNA accumulates around the budding nuclei and is transported into the PGCs. (G-I) After PGC formation the vast majority of the germ plasm is located in the PGCs, only a small fraction remains in the soma (G"-I") with no difference between the genotypes. ( $\left.G^{\prime}\right)$ In wild type embryos almost no osk RNA is detectable in the PGCs, while in maternal lost and in osk-nos-3'UTR embryos osk RNA can be detected within the PGCs.

of the SNUPs. This can be observed in wild type embryos (Fig. $26 \mathrm{G"}$ ), lost embryos (Fig. 26 H") and osk-nos-3'UTR embryos (Fig. 26 I") with no apparent difference between these genotypes. As shown in Figure 25 in wild type embryos the vast majority of osk RNA is present in the soma beneath the PGCs diffusing away from the posterior pole (Fig. $26 \mathrm{G}^{\prime}$ ). Only a small fraction of $o s k$ RNA can be detected within the PGCs (see above for details). In lost embryos the amount of osk RNA which resides within the PGCs is significantly increased (Fig. $26 \mathrm{H}^{\prime}$ ) compared to wild type (see Fig. 25 for details). In osk-nos-3'UTR embryos a substantial amount of osk RNA can be found within the PGCs (Fig. 26 I'). The osk RNA particles which are detected in the soma diffuse away from the posterior pole into the embryo comparable to wild type and lost embryos.

In summary, although osk RNA particles and the germ plasm inhabit the same volume at the posterior cortex before PGCs budding they are regulated differently. In wild type embryos the germ plasm is accumulating around the budding nuclei and by that incorporated into the forming PGCs while osk RNA starts to diffuse away from the cortex. This leaves the vast majority of osk RNA in the soma while the germ plasm is almost exclusively present in the PGCs. In lost embryos an increased amount of osk RNA can be found within the PGCs after their formation. In osk-nos-3'-UTR embryos a part of the osk RNA is accumulating around the budding nuclei and gets transported into the PGC where it is readily detected after PGC formation. This is likely to be the 


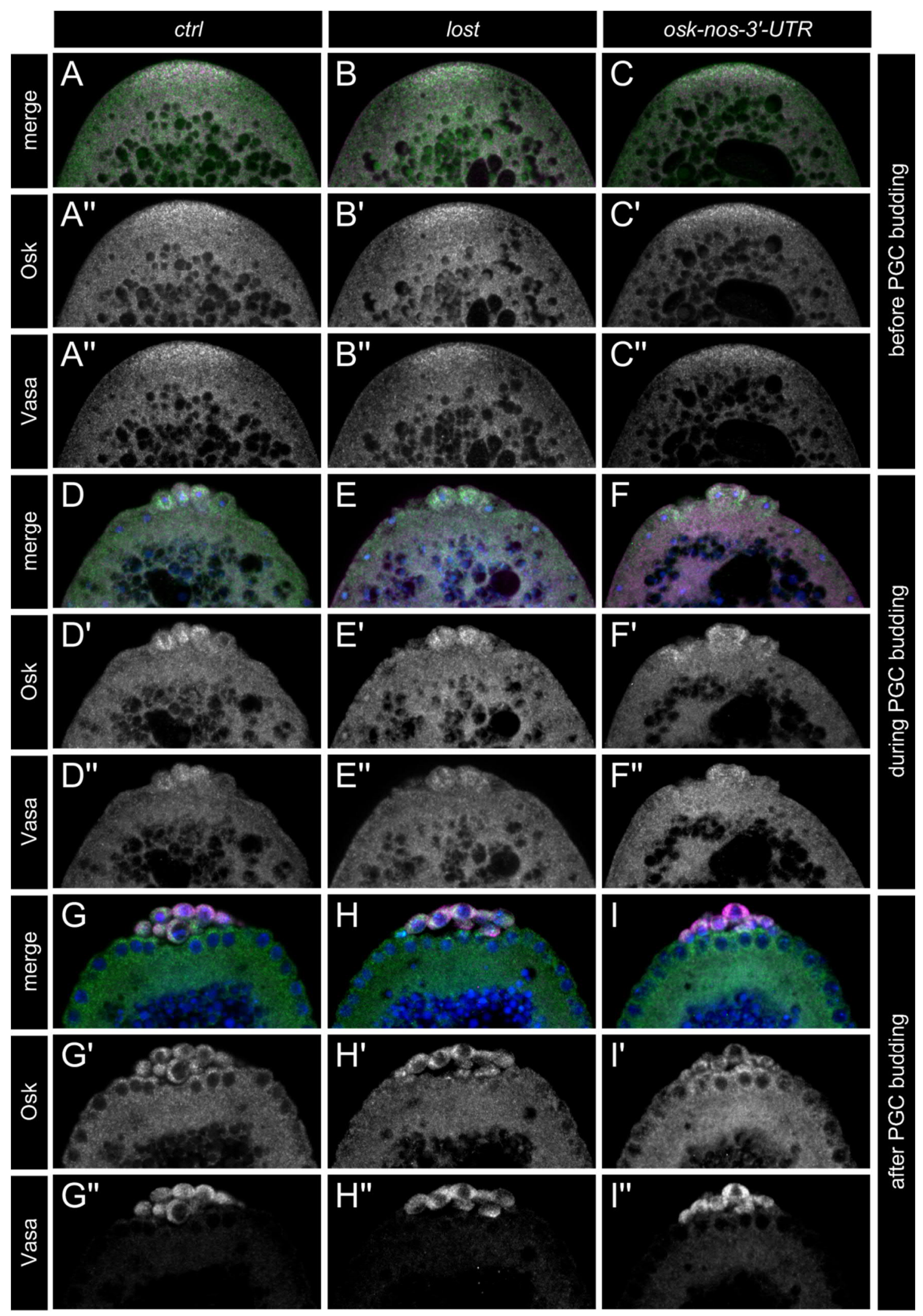


Figure 27: The localization of Osk protein is not affected in maternal lost and in osk-nos3'UTR embryos. (A-I) Maximum intensity projections of 2-4 confocal sections of posterior poles of embryos before (A-C), during (D-F) and after PGC budding (G-I) stained for Vasa (magenta), Osk protein (green) and Dapi (blue). Posterior is up. Localization of Osk protein in maternal lost (B,E,H) and in osk-nos-3'UTR embryos (C,F,I) does not differ from wild embryos (A,D,G). It colocalizes with Vasa and behaves as a germ plasm component: Before PGC budding ( $\left.\mathrm{A}^{\prime}-\mathrm{C}^{\prime}\right)$ Osk resides at the posterior pole, during PGC budding ( $\left.\mathrm{D}^{\prime}-\mathrm{F}^{\prime}\right)$ Osk accumulates around the budding nuclei and after PGC formation $\left(\mathrm{G}^{\prime}-\mathrm{I}^{\prime}\right)$ Osk is mainly found within the PGCs. A small fraction of Osk remains in the soma apical to the SNUPs.

transgenic osk-nos-3'-UTR RNA species. The remaining osk RNA, presumably the endogenous osk RNA molecules, shows a wild type-like localization pattern. This confirms that in embryos from mothers overexpressing osk-nos-3'-UTR osk RNA is indeed ectopically transported into the PGCs.

Like osk RNA Osk protein has been extensively studied during oogenesis and its role as the nucleator for the formation of the germ plasm is well established (reviewed by Lehmann (2016)). Osk protein is a component of the polar granules located at the posterior pole of embryos. These granules also contain the germ line determinants like gcl RNA and Vasa protein. Since osk RNA is mislocalized in lost embryos (see Fig. 25) the question arises whether Osk protein is affected as well. To address these questions the localization of Osk protein during early embryogenesis was visualized using immunofluorescence. The germ plasm was labeled using an antibody against Vasa, Dapi was used to detect the nuclei. Figure 27 shows confocal sections of posterior poles of embryos from wild type mothers (Fig. 27 A,D,G), lost mutant mothers (Fig. 27 B,E,H) and mothers overexpressing osk-nos-3'UTR (Fig. 27 C,F,I) before PGC budding (Fig. 27 A-C), during PGC budding (Fig. 27 D-E) and after PGC budding (Fig. 27 G-I). During the whole process of PGC formation Osk protein resembles the localization pattern of Vasa protein. Before PGC budding both proteins are found at the posterior of the embryo (Fig. 27 A',A") forming, together with other components, the germ granules. During PGC budding both Osk and Vas accumulate around the budding nuclei (Fig. 26 D',D") and are thus transported into the forming PGCs. After PGC budding the majority of both Osk and Vas is present within the PGCs and only a small fraction remains in the soma (Fig. 27 G',G") where it localizes apically of the SNUPs. There are no detectable 
differences in the localization of Osk protein between wild type, lost or osk-nos-3'UTR embryos at any of the analyzed time points. This makes it unlikely that Osk protein is involved in causing the phenotypes observed in lost embryos. Furthermore, the overexpression of osk-nos-3'UTR during oogenesis does not result in changes in Osk protein localization during PGC formation, it merely leads to an increased amount of osk RNA within the PGCs as it is also found in lost embryos (see Fig. 25 and Fig. 26).

\section{Maternal expression of osk-nos-3'UTR is sufficient to cause lost-like} phenotypes

Embryos from lost mutant mothers show a fourfold increase in the relative amount of osk RNA within the PGCs after their formation compared to wild type embryos (see Fig. 25), however, no difference in the amount or localization of Osk protein could be detected (see Fig. 27). This raises the question whether ectopic osk RNA within PGCs can cause the phenotypes observed in lost embryos. To address this question osk-nos-3'UTR embryos were analyzed in detail and the strength of the lost-like phenotypes was quantified. Fixed embryos were stained with an antibody against Vasa to label the PGCs, an antibody against the phosphorylated carboxy-terminal domain (pCTD) of RNApolymerase II to label active transcription and Dapi was used to show the nuclei. Analyses and quantifications were performed as before (see methods for details).

In lost embryos the transcriptional activity of the somatic nuclei underneath the PGCs (SNUPs) is lower than in the other somatic nuclei (see Fig. 15). The same effect can be observed in osk-nos-3'UTR embryos (Fig. 28 A"). A quantification of this phenotype reveals that the average intensity of the pCTD signal within the SNUPs is 0.68 -fold of the signal strength of the other somatic nuclei (Fig. $28 \mathrm{C}$ ). This difference is statistically significant compared to wild type (Mann-Whitney-U test, $* * * p<0.001$ ). The effect seen in osk-nos-3'UTR embryos is as strong as in lost embryos (Fig. $28 \mathrm{C}$ ).

Another feature of lost embryos is an increased relative number of PGCs which show transcriptional activity at stage 6 of development (see Fig. 13). This is also found in osknos-3'UTR embryos (Fig. 28 B"). Quantifying this phenotype reveals that in osk-nos- 

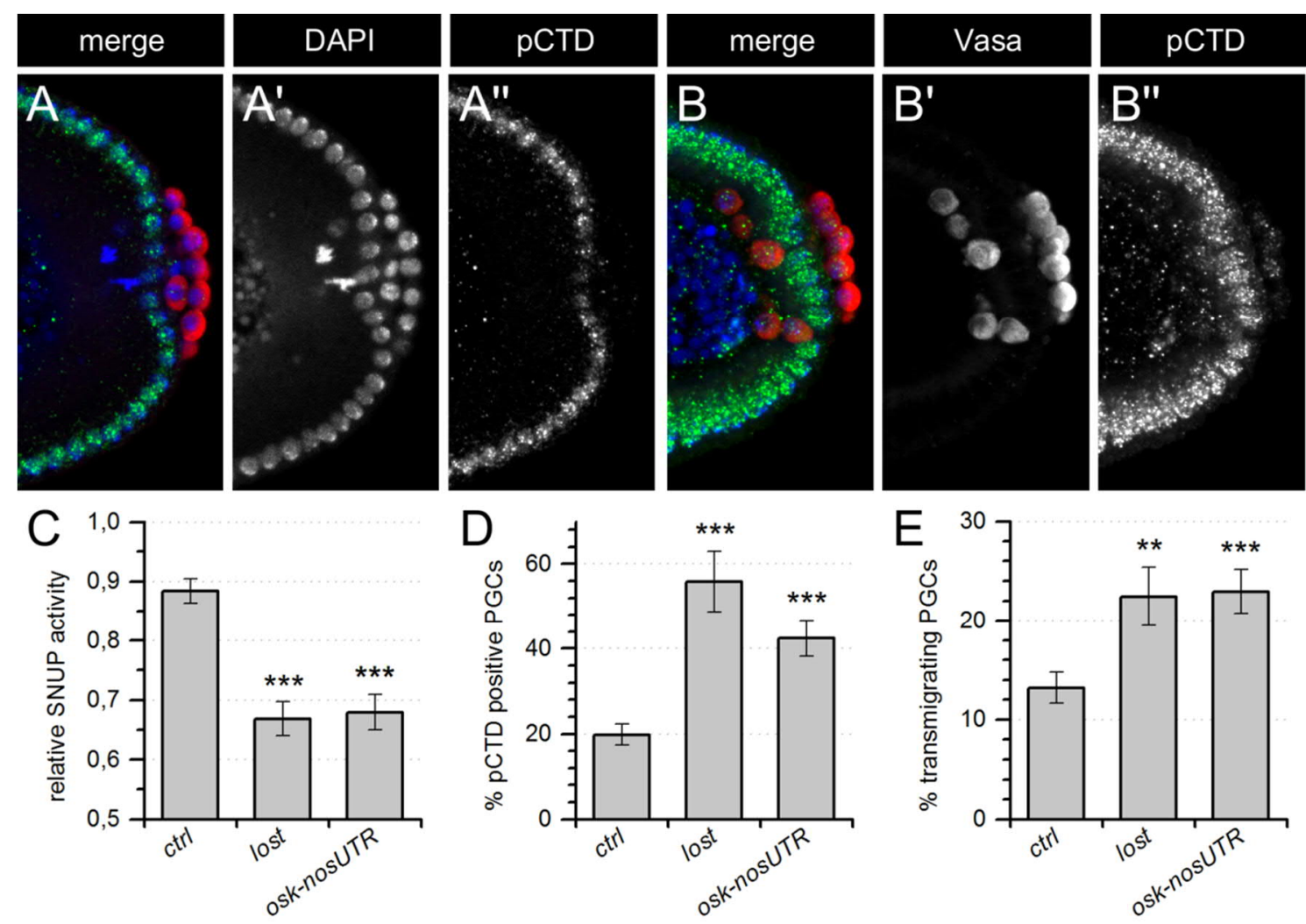

Figure 28: Maternal expression of $o s k-n o s-3^{\prime} U T R$ is sufficient to cause lost-like phenotypes. (A, B) Maximum intensity projections of 2-4 confocal sections of posterior poles of osk-nos-3'UTR embryos stained for Vasa (red), pCTD (green) and Dapi (blue). The presence of a nuclear pCTD signal indicates transcriptional activity. (A) At stage 4 the SNUPs are less transcriptionally active than the other somatic nuclei indicated by their very weak pCTD signal (A"). Dapi (A') labels the nuclei. (B) At stage 6 some of the PGCs, labeled by Vasa (B'), migrate through the epithelium prematurely. (B") Some PGCs show ectopic transcriptional activity. (C) Quantification of the relative transcriptional activity of the SNUPs, (D) transcriptional active PGCs and (D) the premature transmigration in osk-nos-3'UTR embryos in comparison wild type (ctrl) and maternal lost embryos. The columns show the average pCTD signal in the SNUPs relative to the other somatic nuclei (C), the average relative number of pCTD positive PGCs during stage 6 (D) or the average relative number of transmigrating PGCs during stages 6 and 7 (E) of the indicated genotypes. In osk-nos-3'UTR embryos the SNUPs show significantly less transcription (C) while the number of transcriptionally active PGCs and the number of transmigrating PGCs are significantly increased compared to wild type embryos. The phenotypes are as strong as in maternal lost embryos. Error bars show SEM. $* * * \mathrm{p}<0.001$. ** $\mathrm{p}<0.01$ Mann-Whitney-U test.

3'UTR embryos $42 \%$ of the PGCs show transcriptional activity while in wild type embryos only $20 \%$ are transcriptionally active (Fig. 28 D). Although the phenotype is not as strong as in lost embryos (57\%) it differs statistically significant from wild type (Mann-Whitney-U test, $* * * \mathrm{p}<0.001$ ).

In lost embryos an increased number of PGCs migrate through the PMG epithelium during stages 6 and 7 (see Fig. 12). This phenotype can also be observed in osk-nos- 
3'UTR embryos (Fig. 28 B'). A quantification of this phenotype reveals that in osk-nos3'UTR embryos on average $23 \%$ of the PGCs transmigrate (Fig. 28 E), a statistically significant increase compared to $13 \%$ transmigrating PGCs found in wild type embryos (Mann-Whitney-U test, ${ }^{* *} \mathrm{p}<0.01$. ${ }^{* * *} \mathrm{p}<0.001$ ). The effect seen in osk-nos-3'UTR embryos is as strong as in lost embryos (Fig. $28 \mathrm{E}$ ).

This demonstrates that an increased amount of osk RNA within the PGCs caused by transgenic osk-nos-3'UTR RNA is sufficient to induce lost-like phenotypes, including a reduced transcriptional activity in the SNUPs, ectopic transcriptional activity in the PGCs' nuclei and cellularization defects at the posterior pole with transmigrating PGCs. 


\section{Discussion}

The germline cells are of fundamental importance for all animals as they have the ability to give rise to the next generation. Their precursors, primordial germ cells (PGCs), are determined very early during animal development. To form the embryonic gonad they must come together with the somatic gonadal precursor cells (SGPs) which are often specified at a different location within the embryo. This requires the PGCs to actively migrate towards the SGPs.

In Drosophila, the PGCs arise from a specialized cytoplasm, the germplasm, which is deposited at the posterior pole of the oocyte during oogenesis. They form earlier than the somatic epithelium and are kept outside the embryo until gastrulation. Later they start their active migration towards the SGPs in the lateral mesoderm. If they fail to reach their destination they are removed by programmed cell death.

In this thesis two gene functions have been investigated which are required for the early separation of the PGCs from the somatic epithelium. Both genes have overlapping mutant phenotypes but function by completely different mechanisms.

\section{Echinoid}

ed codes for a homophilic cell adhesion molecule that is expressed throughout embryogenesis in all epidermal tissues. $e d$ mRNA is maternally contributed to the early embryo (Kumar et al. 2011) and its RNA and the translated protein are present in the embryonic epithelium during stage 6 but not in the PGCs which reside at the posterior pole of the embryo (Fig. 4). 


\section{Differential expression of Ed prevents PGC transmigration}

Since ed LOF mutations are homozygous lethal the germline clone (GLC) technique was used to obtain maternally ed mutant embryos (Chou et al. 1993). The UAS-Ga14 mediated expression of dsRNA against $e d$ in the female germline (Staller et al. 2013) was used as a completely independent way of obtaining embryos devoid of the maternal contribution of ed RNA. Both, ed GLC and maternal ed RNAi embryos, show the same phenotype: The PGCs, which normally reside on top of the PMG epithelium during embryonic stages 6 and 7, migrate in between the epithelial cells (Fig. 5). This phenotype could be due a role of $\mathrm{Ed}$ in epithelial cell adhesion or cellularization. Several points argue against that: It was shown that in ed mutant clones in larval wing disc epithelia adherens junctions (AJ) between mutant cells are not affected (Wei et al. 2005). Furthermore, an analysis of the localization of the cellularization markers Slam and Dlg in maternal ed RNAi embryos revealed no difference compared to wild type embryos (Fig. 8) ruling out a role of Ed in cellularization. The finding that in embryos lacking maternal ed contribution the PGCs migrate through the PMG epithelium without having cell adhesion or cellularization compromised suggests that the interface between ed-expressing epithelial cells and ed non-expressing PGCs is crucial. Differential expression of $e d$ has been shown to have a function in tissue morphogenesis and has been suggested to drive cell sorting (Lecuit 2005, Laplante and Nilson 2006). This led to the hypothesis that the interface between $e d$-expressing somatic cells and $e d$ non-expressing PGCs prevents intermingling of these two cell populations

Further support for this interpretation comes from embryos in which the expression interface was removed by ectopically expressing $e d$ within the PGCs. This was achieved by maternally expressing a transgene consisting of the ed ORF fused to the 3'UTR of nos in the female germline. In embryos from these mothers ed RNA is enriched at the posterior pole and then in the PGCs (Fig. 6). In these embryos also Ed protein is found in the PGCs as well as in the soma, so the ectopic expression does not influence the endogenous ed expression. These ed-nos-3'UTR embryos show the same PGC transmigration phenotype as maternal ed mutant embryos without affecting 
cellularization. This demonstrates that indeed the differential expression of $e d$ prevents the PGCs from mixing with the underlying epithelial cells.

The results suggest an essential role of the ed expression within the PMG to inhibit the premature transmigration of PGCs. However, during gastrulation and germ band extension (GBE) the PGCs stay in the posterior midgut pocket only up to stage 9 . Then they start to migrate as individual cells in between the epithelial cells and subsequently towards the SGPs in the lateral mesoderm. So if ed expression in the PMG epithelium prevents transmigration a change in Ed localization or expression would be expected at the onset of PGC migration.

Indeed, in wild type embryos Ed remains apically localized in the PMG epithelium until stage 9 when the active migration of the PGCs starts. However, during transmigration Ed is mostly absent from the PMG (Fig. 7). At the same time the PMG epithelium undergoes an epithelial-to-mesenchymal transition (EMT) during which the PMG cells lose their apical-basal polarity and weaken their cell adhesion (Campbell et al. 2011). It is not clear whether Ed is actively removed from the PMG to allow the transmigration or if its absence is a consequence of the EMT. It has been shown, however, that the reduced cell adhesion between the PMG cells is necessary for allowing the PGCs to transmigrate (Seifert and Lehmann 2012). The removal of Ed from the PMG precisely at the time of the PGCs transmigration supports the model that indeed the interface of $e d$ expressing PMG and $e d$ non-expressing PGCs represents a key inhibitory mechanism against an untimed transmigration of PGCs.

\section{Mechanism underling the inhibition of PGC transmigration}

The interface between $e d$-expressing and $e d$ non-expressing cell populations has been studied before. When Laplante and Nilson (2006) introduced ed mutant clones of cells in the follicular epithelium of the egg chamber they found a smooth border between the $e d$ mutant cells and the surrounding $e d$ expressing cells. This borderline is devoid of cell adhesion proteins like Cadherin and Catenin but rich in actin and myosin in the ed 
expressing cells. The same behavior of $e d$ mutant clones in an $e d$ expressing epithelium was reported for the larval wing disc (Wei et al. 2005). The biological relevance of the differential expression of $e d$ has been demonstrated in the context of dorsal closure (Laplante and Nilson 2011). It was shown that Ed is present in the epidermal epithelium but not in the amnioserosa. At the interface, the leading edge, epithelial cells generate an intercellular contractile actomyosin cable which provides physical force that results in a smooth border and was shown to be essential for dorsal closure. If $e d$ is removed from the epithelium or ectopically expressed within the amnioserosa the actomyosin cable fails to form which leads to defects in dorsal closure (Laplante and Nilson 2011).

The ed non-expressing PGCs sitting on top of the ed-expressing epithelium form an interface comparable to that during dorsal closure, although the function of the differential expression of $e d$ is different. While during dorsal closure the differential expression ensures the formation of an intercellular contractile actomyosin cable that stabilizes the leading edge (Laplante and Nilson 2011) in context of the PGCs the differential ed expression prohibits the PGCs mixing with the underlying epithelium. Here, the differential expression of Ed prevents the migration of an overlying cell population through an epithelium. So in contrast to the already described examples of Ed function between $e d$ expressing and non-expressing cells within a planar epithelium where the border resembles a line (Wei et al. 2005, Laplante and Nilson 2006), here a border resembling a plane is generated between a two-dimensional epithelium and a cell population on top.

Interestingly, in fixed wild type embryos an apical actin network is present in the epithelial cells beneath the PGCs (Fig. 9). This network is weakened or disrupted in maternal ed RNAi embryos as well as in ed-nos-3'-UTR embryos indicating that the differential expression of $e d$ is necessary for its formation or stabilization. These findings are confirmed using fly lines which express fluorescently labeled actin and myosin markers in the female germ line (Rauzi et al. 2010). In living embryos with these markers maternally provided a strong apical actin and myosin signal is detected in the epithelium beneath the PGCs (Fig. 10). This network is severely weakened or disrupted in maternal ed RNAi embryos as well as in ed-nos-3'-UTR embryos ectopically expressing $e d$ in the PGCs (Fig. 10). 
Although the interface between $e d$ expressing and non-expressing tissues promotes the formation of actomyosin cables both during dorsal closure and in the PMG cells underneath the PGCs the situation is slightly different: The PGCs are not in the same plane as the epithelium but on top of it. This also means that the interface between $e d$ expressing and ed non-expressing cells is not a line as in the follicle but a plane. Accordingly the actomyosin forms an intercellular network at the apical side of the epithelial cells and not a cable. However, it is not clear whether it resembles an apicolateral actomyosin connected to the adherens junctions (AJ) or a subapical or apical cortical network.

The germ band extension (GBE) further complicates the situation. This process is mainly driven by cell intercalation and cell shape changes (Irvine and Wieschaus 1994, da Silva and Vincent 2007). These tissue movements are taking place without compromising epithelial integrity which requires the constant remodeling of AJ. The role of actomyosin cables during these processes have been studied in detail during the invagination of the mesoderm at embryonic stage 6 (Coravos and Martin 2016) as well during GBE (Rauzi et al. 2010). While DE-Cad in the AJ keeps the cells attached actomyosin is providing the force for the morphogenetic movements. Two distinct actin systems have been described to play a role: An actin cable located in the subapical cortex which is connected to the AJ and an actomyosin meshwork in the apical cortex (Martin et al. 2009). The former plays a role in epithelial integrity and positioning of the AJ (Lecuit and Lenne 2007) while the latter provided physical force for cell shape changes like apical constriction (Coravos and Martin 2016). During the movement the local concentration of myosin and therefore the force generation is highly dynamic (Mason et al. 2013). It is likely that similar mechanisms are used by the cells of the PMG to regulate their adhesion shape during GBE which is reflected by the strong accumulation of actin in the apical region of PMG cells which starts to undergo cell shape changes during GBE (Fig. 9, 10).

It is, however, unclear which of the actomyosin systems is used by the differential expression of $e d$. No conclusion can be drawn from analyzing the confocal images 
because both actomyosin populations are located apically in the cells and are therefore in close proximity. Furthermore, the PGCs which are located directly on top of the epithelium also contain actin and myosin. By analyzing the confocal images it is impossible to distinguish to which of two touching cells the actin signal detected at the interface belongs. It is, however, unlikely that Ed affects the DE-Cad associated species of actin because it has been published that $e d$ LOF has no impact on cell adhesion (Wei et al. 2005). Furthermore, GBE and gastrulation are not disturbed neither in maternal ed RNAi nor in ed-nos-3'UTR embryos which argues against Ed affecting AJ. It has been shown, however, that DE-Cad is linked to the actin cytoskeleton via $\alpha$-Catenin and Girdin (Gir) while Ed uses Canoe (Cno) as an adaptor (Wei et al. 2005, Desai et al. 2013, Houssin et al. 2015). Further research focusing on these genes might help to shed light on the molecular mechanisms by which Ed is affecting the actin cytoskeleton.

However, it cannot be excluded that the differential expression of ed triggers the formation of another, distinct cortical actomyosin network in the PMG epithelium. It has been shown that physical tension provided by cortical actomyosin can drive cell sorting and morphogenesis (Umetsu et al. 2014, Duque and Gorfinkiel 2016). Recently it was shown that in early mouse blastocysts the cells that become the inner cell mass sort themselves out from the later trophectoderm cells via differential surface tension (Samarage et al. 2015). Live imaging and mathematical models show that cortical tension also directs cell sorting during zebrafish gastrulation (Krieg et al. 2008). Cortical tension provided by actomyosin seems to be commonly used mechanism for cell sorting in animal development. However, the means of regulating the actin cytoskeleton differ between species. 


\section{Lost}

lost codes for a highly conserved RNA binding protein which has been found in several RNA-protein complexes, such as splicosomes and sponge bodies (Herold et al. 2009, Snee and Macdonald 2009). Furthermore, it has been shown to form a complex with Rumpelstiltskin (Rump) during oogenesis which directly binds to nos mRNA and has a function for transport and anchoring of the germ plasm (Jain and Gavis 2008, Sinsimer et al. 2011). Lost protein and RNA are maternally provided to the early embryo (Molitor 2002). After the maternal-to-zygotic transition (MZT) the lost RNA is degraded in the somatic part of the embryo and stabilized in the PGCs (Molitor 2002). However, Lost protein is localized ubiquitously in the embryo but slightly enriched at the posterior pole before PGC formation and later in the PGCs (Fig. 11).

\section{$\underline{\text { Posterior and PGC development is affected in maternal lost mutants }}$}

In embryos derived from mothers that are homozygous mutant for lost (hereafter referred to as maternal lost mutant embryos) some PGCs traverse the PMG epithelium prematurely during embryonic stages 6 and 7 through gaps in the epithelium. This phenotype solely depends on the genotype of the mother (Hertel 2011) therefore only maternal mutants have been analyzed here. Although Lost is present throughout the embryo the epithelial integrity is compromised only in somatic cells directly beneath the PGCs. Staining of various proteins expressed in the somatic epithelium revealed a normal expression and localization except for the area beneath the PGCs. Marker proteins for the apical, lateral and basal membrane domains show a reduced expression level and are localized abnormally only in these somatic cells (Fig. 14). This argues against a general function of Lost for cellularization or cell adhesion between the somatic epithelial cells and supports a model of specific function of Lost in the region of the posterior pole of the embryo. 
Shortly before cellularization the somatic nuclei are characterized by a uniformly strong transcriptional activity as judged by an antibody against active RNA polymerase II (Fig. 15, Palancade and Bensaude (2003)). In contrast, the PGCs are mostly transcriptionally silent after their formation at stage 3 until they start their active migration at stage 9 in wild type embryos (Fig. 13, Zalokar (1976)). In maternal lost mutants, however, the transcriptional activity of the somatic nuclei underneath the PGCs (SNUPs) is reduced to about two thirds compared to the other somatic nuclei. This local reduction in transcriptional activity is perfectly overlapping with the region of the observed changes in expression and localization of epithelial marker proteins.

It has been demonstrated that active zygotic transcription is essential for cellularization (Edgar et al. 1986), thus the reduced transcriptional activity in the SNUPs observed in maternal lost mutant embryos could cause the cellularization phenotypes observed at the posterior pole. In wild type embryos the PGCs migrate through the PMG epithelium during stages 9 and 10 while at the same time the PMG undergoes an epithelial-tomesenchymal transition (EMT) weakening its cell adhesion (Campbell et al. 2011). It has been shown that compromising epithelial integrity (either by EMT or other means) is necessary for allowing the PGCs to transmigrate (Seifert and Lehmann 2012). The premature transmigration seen in maternal lost mutant embryos could therefore be explained by compromised epithelial integrity at the posterior pole which is a result of cellularization defects caused by reduced levels of zygotic transcription in the SNUPs.

The analysis RNA polymerase II activity not only revealed the described reduction of the activity in the somatic cells beneath the PGCs, but also that the number of transcriptional active PGCs is increased 3-fold in maternal lost mutant embryos (Fig. 13). In wild type embryos the PGCs are mostly transcriptionally silenced after their formation (Seydoux and Dunn 1997). This is a general feature of developing PGCs and has been shown in mice, worms, flies and others (Leatherman and Jongens 2003, Nakamura and Seydoux 2008, Strome and Updike 2015). The mechanisms by which the PGCs shut down their transcription differ among species. In Drosophila the PGC silencing is mediated by Pgc, a 71 amino acid peptide (Hanyu-Nakamura et al. 2008), which directly binds to the positive Transcription Elongation Factor b (pTEFb) thereby 
preventing its recruitment to chromatin (Hanyu-Nakamura et al. 2008). Therefore, pTEFb is unable to phosphorylize the carboxy-terminal domain (CTD) of RNA polymerase II, which is necessary for active transcription (Dahmus 1996). If the transcriptional silencing is disturbed in Drosophila the PGCs show developmental defects and eventually die (Nakamura et al. 1996). Although the PGCs are transcriptionally active in maternal lost mutants such an excessive cell death is not seen in maternal lost mutants (Hertel 2011). This might be due to the magnitude of the disruption of the transcriptional silencing. In maternal $p g c$ mutants or in maternal $p g c$ RNAi embryos $85 \%$ or $98 \%$ of the PGCs, respectively, are transcriptionally active (Fig. 20) and in maternal lost mutants only 54\% of the PGC. The analysis of PGC cell death is further complicated by the fact that those PGCs that migrate through the PMG prematurely are removed by programmed cells death as well.

In wild type the PGCs start their active migration at embryonic stage 9 (Jaglarz and Howard 1995). At the same time they become transcriptionally active (Zalokar 1976). Therefore, it could be that active transcription is a prerequisite for active migration since both phenomena are seen in maternal lost mutants. Two findings argue against this hypothesis. First, it has been shown that PGCs in wild type embryos, which are transcriptionally silent after their formation, are highly motile (Kunwar et al. 2008). Second, there is no correlation between transcriptional activity and transmigration at stage 6. The transmigrating PGCs are not necessarily transcriptionally active, while the transcriptionally active PGCs are not necessarily transmigrating (Fig. 13). This supports the view that PGCs have the ability to transmigrate independently of their transcriptional state strongly supporting the model that the two phenotypes observed in maternal lost mutants are independent.

\section{Lost affects the localization of osk RNA}

In summary, all lost mutant phenotypes (premature transmigration of the PGCs, reduced transcriptional activity of the SNUPs, ectopic transcriptional activity of the PGCs) are restricted to somatic cells beneath the PGCs or directly involve PGCs. Lost protein, however, is present throughout the embryo. This discrepancy indicates that Lost protein 
must have a specific function at the posterior pole and/or in the PGCs during the early embryonic stages. Since Lost was found in various RNA-protein complexes (Herold et al. 2009, Snee and Macdonald 2009), it has a putative RRM and it was suggested to play a role in germ plasm transport during late oogenesis (Sinsimer et al. 2011) a function of Lost in regulating germ plasm RNA transport or anchorage during early embryogenesis was investigated. While FISH analysis revealed no difference in the localization of $c y c B$, nos, $p g c$ and $g c l$ RNA between lost mutants and wild type during stage 1 and stage 4 the amount of $o s k$ RNA within the PGCs was significantly increased in maternal lost mutant embryos compared to wild type embryos at stage 4 (Fig. 25).

It was shown that, in contrast to other germ plasm RNAs, osk RNA is not detectable in the PGCs after their formation (Little et al. (2015), Fig. 25) although it is present at the posterior pole of the embryo before the PGCs form. In wild type embryos only a fraction of osk RNA is incorporated into the PGCs while the majority remains in the soma (Fig. 26). The osk RNA in the PGCs then is rapidly degraded in wild type embryos. In contrast, osk RNA can be reliably detected in the PGCs in maternal lost mutants (Fig. 25, 26). However, the experimental design does not allow determining whether the increased amount of osk RNA in PGCs originates from a larger fraction of the osk RNA originally transported into the PGCs or from its reduced degradation in lost mutant embryos.

To investigate whether the observed increased amount of osk RNA in the PGCs causes the lost mutant phenotypes a transgenic fly line was obtained which maternally expresses an osk RNA variant which lacks its own localization signals and is fused to the 3'-UTR of nos (Little et al. 2015). The 3'-UTR of nos targets the transgenic osk RNA into the PGCs (Van Doren et al. 1998b). Embryos from mothers expressing osknos-3'UTR RNA during oogenesis indeed show the same phenotypes as maternal lost mutant embryos: The PGCs migrate through the PMG epithelium prematurely, the SNUPs have reduced transcriptional activity and the PGCs become transcriptionally active (Fig. 28). This independent experimental design proves that targeting osk RNA into the PGCs is sufficient to cause lost-like phenotypes both in the PGCs and in the somatic cells beneath them. Furthermore, since the localization of the osk-nos-3'-UTR 
RNA into the PGCs is sufficient to induce the observed mutant phenotypes a role of the 3'UTR of the osk RNA can be excluded. This result supports the model that the mislocalization of an RNA that contains the ORF of Osk is sufficient to cause the phenotypes.

Although osk RNA is degraded within the PGCs in wild type embryos Osk protein is present in the PGCs where it is part of the germ granules (Trcek et al. (2015), Fig. 27). Surprisingly, PGCs of maternal lost mutant embryos, which have an increased amount of osk RNA, do not show elevated levels or mislocalization of Osk protein (Fig. 27). The same holds true for embryos from mothers expressing osk-nos-3-UTR, which targets the transgenic osk RNA into the PGCs in high amounts (Fig. 27). Although Osk protein levels could only be measured in a semi-quantitative way, these results support the conclusion that the lost-like phenotypes in these embryos are caused by the excess of osk RNA within the PGCs independent of the presence of increased levels of Osk protein. Therefore, increased amounts of the osk RNA including the ORF without a requirement of additional Osk protein translation seem to be the cause for the observed phenotypes in lost mutants.

\section{Osk protein and osk RNA are part of different RNP particles}

Osk protein induces the formation of the germ plasm during oogenesis. osk RNA is transcribed in the nurse cells and transported into the developing oocyte. During the transport its translation is inhibited until it reaches the posterior pole of the oocyte, where Osk protein is produced. Several regions of the osk RNA, located in the ORF and in the 3'-UTR, have been shown to be essential for its localization and translational regulation (Jambor et al. 2011, Simon et al. 2015). Osk protein then binds its own RNA resulting in a positive feedback loop which further increases the amount of Osk protein at the posterior pole. Osk protein also recruits Vasa and Tudor and by that induces the formation of the germ plasm at the posterior pole of the oocyte (Breitwieser et al. 1996, Anne 2010). 
However, less is known about the function of osk RNA. Recently it was reported that osk RNA has the ability to rescue certain osk mutations in trans during oogenesis (Macdonald et al. 2016). It was shown that in ovaries without wild type osk RNA two osk RNA species with independent mutations can rescue each other when the mutations are positioned in different domains of the RNA. This ability to rescue in trans could be blocked by preventing the formation of RNPs indicating that it is sufficient that all essential osk RNA regions are present in a single RNP rather than on a single RNA molecule. They also showed that osk RNA molecules affect other RNA molecules which are present in the same RNP (Macdonald et al. 2016). The authors suggest that osk RNA molecules recruit certain proteins to the RNP which in turn could regulate the behavior of all the RNA molecules within that RNP (Macdonald et al. 2016).

These results show an essential role of osk RNA for the formation and composition of RNPs thereby regulating the transport and translation of additional RNAs. Some RNAs recruit factors to the RNP which affect the localization and/or translation of all the RNA species in that RNP (Macdonald et al. 2016). Therefore interfering with the composition of RNPs by mutating RNAs or proteins affect several genes whose RNA happens to be part of the affected RNPs. This might result in mislocalized RNAs or untimely or ectopic translation or ectopic translational repression.

RNPs and their dynamic composition are known to be of great importance for the regulation of transport and translation of RNAs (Stoiber et al. 2015). Recent advances in imaging and image analysis allow a more detailed investigation of RNPs during Drosophila oogenesis and early embryogenesis. Little et al. (2015) reported that the number of nos RNA molecules within a single RNP increases from 1 during transport to up to 60 (with a mean of 16) once the particles reach the posterior pole of the oocyte. This indicates that single RNA molecules get incorporated into large RNPs at the posterior pole. $p g c$ and $c y c B$ RNAs behave in a similar way: After traveling as singlecopy RNPs they assemble into homotypic clusters at the posterior pole of the oocyte. These clusters (containing either nos, $p g c$ or $c y c B$ ) then aggregate to form the germ granules (Little et al. 2015). However, it was also shown that osk RNA behaves in a fundamentally different way. osk RNA forms multi-copy particles already during 
transport and is assembled into large aggregates containing 50 to 250 molecules of osk RNA at the posterior pole. Furthermore, the osk RNA particles are devoid of $n o s, c y c B$ and Vasa and are not transported into the PGCs during embryogenesis (Little et al. 2015).

Similar results were published by the group of Ruth Lehmann (Trcek et al. 2015). They combined single molecule FISH with super resolution microscopy and found two distinct species of RNA-protein particles at the posterior pole of early embryos. One, the germ granule, contains the well characterized germ line determinants (Vasa, Osk, Tud, $g c l, c y c B, n o s, p g c$ ) while the other particle contains osk RNA but no Osk protein (Trcek et al. 2015). The fact that in contrast to the germ granules osk RNA is not actively transported into the PGCs suggests that these two particles are regulated differently.

The only germ plasm RNA that has been found in both types of RNPs is pgc (polar granule component) RNA with around $50 \%$ of the $p g c$ RNA molecules being part of the osk RNP (Trcek et al. 2015). Therefore, pgc RNA in the osk RNA RNP would be a good candidate that could be affected by the observed mislocalization of osk RNA into the PGCs.

A detailed analysis of the distribution of germ plasm RNAs in stage 1 wild type embryos revealed that $p g c$ and nos RNA show a weaker staining intensity at the center of the germ plasm compared to the outer area of the germ plasm in early wild type embryos (Fig. 16, 17). This finding is highly reproducible and can also be observed in the genome-wide FISH database (Lecuyer et al. 2007). However, in other publications visualizing nos RNA in early embryos using a different staining procedure this reduced staining intensity is not seen (Little et al. 2015). This indicates that the reduced signal intensity in the center of the germ plasm is not due to a reduced number of RNA molecules but a result of the staining method. This interpretation is supported by Slaidina and Lehmann (2017), who found the concentration of germ plasm components to be the highest in the center of the germ plasm. It might be that the reduced signal intensity is caused by a very tight packaging of the respective RNA molecules into 
RNPs in the center of the germ plasm. This could restrict the ability of the antisenseRNA probe to bind to its target when certain FISH staining methods are used. Interestingly, when early wild type and maternally lost mutant embryos are stained and imaged using identical methods the reduction of FISH staining intensity for $p g c$ RNA is less strong in lost mutants compared to wild type embryos (Fig. 18). Assuming that the reduced signal intensity is caused by the packaging of the RNA the observed phenotype could be explained by an altered structure or composition of the $p g c$ containing RNPs in maternal lost mutants.

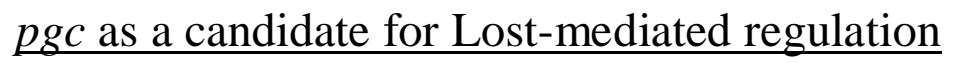

$p g c$ is a well characterized germ plasm RNA. It codes for a 71-amino acid peptide which binds to positive Transcription Elongation Factor b (pTEFb). This prevents pTEFb from phosphorylating RNA-polymerase II and by that globally inhibits transcription (Hanyu-Nakamura et al. 2008). Interestingly, maternal lost mutant embryos combine features found in $p g c$ LOF and $p g c$ GOF embryos. Like in embryos from mothers devoid of $p g c$ the transcriptional silencing of the PGCs is disrupted, i.e. active transcription can be detected in the nuclei of the PGCs (Hanyu-Nakamura et al. (2008), Fig. 20). In pgc GOF embryos (from mothers having 6 genomic copies of the pgc locus instead if 2) not only the PGCs get transcriptionally repressed but also the somatic nuclei at the posterior pole of the embryo which leads to the "pole-hole" phenotype where some PGCs "fall" into embryo (de Las Heras et al. 2009). Exactly these features are also seen in lost embryos: The somatic nuclei underneath the PGCs (SNUPs) show less active transcription than the other somatic nuclei (Fig. 15). The epithelial integrity is compromised only beneath the PGCs (Fig. 14) which allows them to transmigrate through "holes" in the epithelium (Fig. 19).

This combination of features from pgc LOF and pgc GOF phenotypes in lost mutants could be explained by a misregulation of $p g c$ RNA mediated by $o s k$ RNA. pgc and $o s k$ RNA can be found in the same RNP in early embryos (Trcek et al. 2015) and osk RNA is affected in maternal lost mutants. Furthermore, it has been demonstrated that the presence of osk RNA in an RNP can influence other RNAs in the same particle 
(Macdonald et al. 2016). A misregulation of $p g c$ transport or translation could therefore lead to less $p g c$ activity within the PGCs (explaining the ectopic transcriptional activity) and more $p g c$ activity remaining in the soma (explaining the reduced transcriptional activity in the SNUPs and the transmigration) in maternal lost mutant embryos. A detailed analysis of the distribution of $p g c$ RNA between soma and PGCs in wild type embryos and lost mutants, however, revealed no clear difference (Fig. 22). Within the limitations of the semi-quantitative measurement of the $p g c$ RNA amount this result rules out an important function of Lost for the transport of $p g c$ RNA into the PGCs.

Since no difference in the distribution of $p g c$ RNA between soma and PGCs after PGC formation was found a misregulation of $p g c$ translation due to changes in RNP structure or composition mediated by osk RNA could alternatively explain the phenotypes observed in maternal lost mutants. The increased amount of osk RNA within the PGCs of lost mutant embryos could negatively affect the translation of $p g c$ by recruiting translational repressors to the shared RNP. The weaker reduction in $p g c$ FISH staining intensity at the center of the germ plasm in maternal lost mutant embryos at stage 1 could be due to a less dense structure of the $p g c$ containing RNPs which could result in ectopic translation of $\mathrm{pgc}$ in the soma.

The translation of $p g c$ is tightly regulated: It is only translated after the PGCs have formed to prevent transcriptional silencing of the somatic nuclei (Rangan et al. 2009). If Lost is a part of a $p g c$-containing RNP it might have an influence on its translation. Lost might affect the temporal and/or spatial regulation of translation by influencing the physical properties or the composition of the RNP. As discussed above, Lost affects the localization of osk RNA after PGC formation. Furthermore, it was shown that about 50\% of the pgc RNA in early embryos are part of the osk RNP (Trcek et al. 2015). Interestingly, the fraction of $p g c$ RNA which colocalizes with osk RNA is reduced at the same time the germ plasm gets transported into the forming PGCs and the translation of $p g c$ starts (Trcek et al. 2015). This means that either osk RNA is removed from the RNP or that $p g c$ RNA leaves the RNP. Since it was also shown that osk RNA can affect the translation of other RNA molecules in the same RNP (Macdonald et al. 2016) it could 
be that the translation of $p g c$ is regulated via osk RNA. This raises the possibility that Lost could have an indirect effect on the translation of $p g c$ via influencing osk RNA.

If Lost affects the spatial and/or temporal regulation of $p g c$ translation there should be an excess of Pgc peptide detectable in the soma after PGC formation. Comparative analyses of antibody stainings of wild type and maternal lost mutant embryos, however, did not show any noticeable difference in the amount or localization of Pgc peptide (Fig. 23). However, the low quality of the antiserum did not allow the detection of subtle changes of Pgc expression rates. Therefore, an independent way of visualizing the translation of $p g c$ was used. A maternally expressed transgenic construct consisting of the ORF of GFP and the 3'-UTR of $p g c$ allows the analysis of the translation of $p g c$ (Rangan et al. 2009). It was shown that the expression of GFP from this construct recapitulates the translation of $p g c$ RNA both temporally and spatially due to the regulatory signals being localized in the 3'-UTR. However, comparison of anti-GFP stainings derived from this construct in wild type and in maternal lost mutant embryos carrying this transgenic construct did not show any clear difference neither in the amount or localization of GFP expression nor its temporal expression (Fig. 23). However, the relatively high levels of background noise in both cases make it impossible to detect or exclude subtle changes in the amount or localization of Pgc peptide or the GFP reporter. An improved staining procedure and the use of better antibodies could increase the signal-to-noise ratio and may make it possible to detect even small differences between wild type and lost mutant embryos in the future.

\section{The pgc 3'UTR can cause lost-like phenotypes}

A detailed analysis of PGCs in embryos from mothers overexpressing GFP-pgc-3'UTR, however, revealed that these embryos show the same phenotypes as maternal lost mutant embryos (Fig. 24): The PGCs transmigrate prematurely through gaps between the epithelial cells and the PGCs show active transcription. These results strongly suggests that an excess of RNA including the 3'-UTR of pgc without its ORF can cause the same defects at the posterior pole as the lack of Lost and as an excess of osk RNA in 
the PGCs. It was shown before that the 3'-UTR of $p g c$ dictates the spatial and temporal regulation of $p g c$ translation (Rangan et al. 2009). It is most likely that this regulatory activity is based on recruiting proteins which then ensure its proper localization and translation. Therefore, overexpressing GFP-pgc-3'-UTR RNA might result in a competition of the transgenic $p g c-3$ '-UTR with the endogenous one for the regulating factors. This could lead to a less efficient transport or a less tightly controlled translation of the endogenous $p g c$ RNA species which in turn would cause the observed phenotypes. The phenomenon that certain RNAs can affect other RNA species by competing for regulatory factors was already described during oogenesis, where the lack of osk RNA causes early oogenesis defects, because the translational repressor Bruno (Bru), which normally binds osk, was free to block the translation of other RNAs (Kanke et al. 2015). However, it is not known which factors regulate the translation of $p g c$ RNA. Therefore, it remains unclear whether the translation of $p g c$ and of $o s k$ RNA is controlled by overlapping activities.

As discussed above, the overexpression of GFP-pgc-3'UTR causes lost-like phenotypes with a combination of $p g c \mathrm{LOF}$ and $p g c$ GOF features, so the expression of the $p g c$ 3'UTR does not simply shift the wild type conditions into one direction. In contrast, embryos from mothers bearing 6 copies of the genomic pgc locus instead of 2 only show strong $p g c$ GOF phenotypes: An excess of $p g c$ RNA can be found in the soma after PGC formation, the SNUPs have dramatically reduced levels of transcription causing cellularization defects and later premature transmigration of the PGCs. The transcriptional silencing of the PGCs, however, is not affected. Therefore, the overexpression of the $p g c$ 3'UTR together with its ORF results in the compensation of the phenotype within the PGCs (the ectopic transcriptional activity in the PGCs). This results clearly shows that the lost-like phenotypes are only dependent on the pgc 3'UTR.

\section{Hypothetical model}

In summary, two separate phenotypes are found in maternal lost mutant embryos. First, the increased transmigration of the PGCs, due to cellularization defects at the posterior pole caused by reduced transcriptional activity in the SNUPs. Second, the 
transcriptional silencing of the PGCs is disrupted so that the number of transcriptionally active PGCs is increased fourfold. The same two phenotypes are observed in embryos from mothers which express GFP-pgc-3'UTR or osk-nos-3'UTR in their germline. Both defects have been described independently in conjunction with $p g c$. The first phenotype, transmigrating PGCs and less transcription in the SNUPs, is found in pgc GOF embryos, while the second phenotype, transcriptionally active PGCs, is seen in pgc LOF embryos. Therefore, both effects could be explained by a disturbed regulation of the activity of $p g c$ : the somatic nuclei experience an excess of $p g c$ activity and the PGCs lack $p g c$ activity. Both, osk and $p g c$ RNA, are transported and localized as RNPs. Furthermore, Lost binds to RNA directly or indirectly (via Rump) and has been found in several RNPs. It is therefore likely that the lost mutant phenotypes are caused by a misregulation of RNPs, their localization and/or their physical properties. However, no change in pgc RNA or peptide localization could be observed in maternal lost mutants, suggesting that either these changes are very subtle or that the activity of $p g c$ can be regulated independent of RNA and protein amounts.

pgc RNA is part of the germ plasm located at the posterior pole and is then transported into the PGCs. It is not translated before PGC formation to avoid silencing the transcription of the somatic nuclei. The mechanisms regulating the translation of $p g c$ are unknown, however its 3'UTR is sufficient to control its temporal and spatial translation (Rangan et al. 2009). pgc RNA is present in two distinct RNPs in early embryos, the germ granule and the osk RNP (Trcek et al. 2015). It might therefore be that the premature translation of $\mathrm{pgc}$ is inhibited by two different mechanisms dependent on the type of RNP. Lost could be a part of these RNPs and directly or indirectly bind pgc RNA and affect its translation.

As a germ granule component, Lost might promote a tightly packed structure (suitable for storing and transporting) of $p g c$ RNA molecules within the germ granules when their concentration is high. It has been shown that the concentration of germ plasm components is higher in the center of the germ plasm that in its marginal regions (Slaidina and Lehmann 2017). Lost protein is also slightly enriched in the center of the germ plasm (Fig. 18). The fact that the $p g c$ FISH staining intensity is lower at the center 
of the germ plasm although there is more $p g c$ RNA might be due to a very tight packaging of the RNA molecules. A tight packaging of the RNP might also prevent the premature translation of $p g c$. To date it is not known how the translation of germ granule components is regulated in detail. However, it was shown that different RNAs of the germ plasm are translated at different time points depending on their 3'UTR sequences (Rangan et al. 2009). One regulatory mechanism could be a very tight clustering of the $p g c$ RNA molecules in the germ granules before PGC formation. Once the germ granules enter the PGCs they dissolve and release the germ line determinants ( $p g c$ RNA being one of them) and allow their translation. In the absence of Lost the $p g c$ RNA molecules might be less tightly packed which would explain the increased pgc FISH staining intensity observed in maternal lost mutant preblastoderm embryos. This might lead to precocious translation of $p g c$ in the soma which in turn leads to less zygotic transcription in the SNUPs (as observed) and later to cellularization defects and transmigrating PGCs.

About $50 \%$ of the $p g c$ RNA are not part of the germ granules but inhabit the same RNP as osk RNA. This population of pgc RNA molecules might be regulated by other mechanisms. osk RNA has been shown to recruit translation repressors like Bru and by that affect the translation of other RNA species which are in the same RNP (Macdonald et al. 2016). So the translation of $p g c$ would be indirectly inhibited by osk RNA. This model is supported by the fact, that before the $p g c$ RNA is transported into the PGCs (where it is supposed to be translated) it loses its connection with osk RNA (Trcek et al. 2015), which remains in the soma. It is not known, however, whether osk RNA is removed from the particle or $p g c$ RNA and how this process is regulated. It might be that Lost promotes the separation of $p g c$ RNA from the osk RNP. In maternal lost mutants osk RNA could therefore be transported into the PGCs along the pgc RNPs. This would explain the increased amount of osk RNA in the PGCs which is observed in maternal lost mutant embryos. This model could also explain the impaired transcriptional silencing of the PGCs. If osk RNA is still present in the same RNP as $p g c$ in the PGCs it might inhibit the translation of $p g c$ (via recruiting for Bru or other factors to the shared RNP) which in turn leads to the observed ectopic transcriptional activity of the PGCs. 
The same phenotypes as in maternal lost mutants are also observed in embryos from mothers which express GFP-pgc-3'UTR or osk-nos-3'UTR during oogenesis. A maternal overexpression of the 3'-UTR of pgc might result in the competition of the transgenic RNA and the endogenous $p g c$ RNA for Lost and other putative regulatory factors. This would explain why an excess of $p g c-3$ 'UTR phenocopies the maternal lost mutants.

The very same phenotypes are also caused by maternal overexpression of osk-nos3'UTR, although the mechanism has to be a different one. First, the transgenic osk RNA might compete with the endogenous osk RNA species for translational inhibitory factors like Bru or others. This would lead to a reduced amount of translational inhibitors bound to the endogenous osk RNP, which in turn could lead to premature translation of $p g c$ from the osk RNP. This model would explain why an excess of $o s k$ RNA causes the reduced levels of transcription in the SNUPs and the premature transmigration of the PGCs. Second, the transgenic osk RNA and with it the putative translational inhibitors are part of the germ granules. There they might associate with the endogenous $p g c$ RNA. Before PGC formation this does not have any effect, but after PGC formation this might result in the less efficient translation of $p g c$ and therefore impaired silencing. This model would explain how the maternal overexpression of osk-nos3'UTR can cause the observed ectopic transcriptional activity in the PGCs. 


\section{Methods}

Here the methods are briefly explained. For detailed protocols see Appendix I: Protocols and Appendix II: Fiji macros.

\section{Fly methods}

\section{Embryo collection and fixation with PFA}

Flies were grown on standard yeast-corn-meal-molasses food at $18^{\circ} \mathrm{C}$ or $25^{\circ} \mathrm{C}$. Embryos of the desired developmental time window are collected on apple juice agar plates supplied with yeast paste. Fixation is performed as described (Karr and Alberts 1986) with minor modifications. Embryos are transferred from the plates to embryo collection sieves and thoroughly rinsed with water. Then they are incubated in 50\% bleach solution for $3 \mathrm{~min}$. After rinsing with water the embryos are transferred to a $20 \mathrm{ml}$ scintillation vial containing $6 \mathrm{ml}$ heptane and $1.5 \mathrm{ml}$ fixation solution and incubated for $20 \mathrm{~min}$ on a shaker. After removing the lower phase $5 \mathrm{ml}$ of $\mathrm{MeOH}$ are added, followed by vigorous manual shaking the vial for 20-30sec. The successfully fixed and devitellinized embryos sink to the bottom of the vial and the upper phase is removed. Then the embryos are rinsed 3 to 5 times with $\mathrm{MeOH}$, transferred to a $1.5 \mathrm{ml}$ test tube and stored at $-20^{\circ} \mathrm{C}$.

\section{$\underline{\mathrm{MeOH}-\text { free devitellinization }}$}

Some staining procedures require fixed and devitellinized embryos without the use of $\mathrm{MeOH}$. For that the embryos are collected and fixed as described above using a higher concentration of PFA and longer incubation. After the fixation the lower phase (PFA) is removed, then the upper phase (heptane) is removed leaving the embryos in the vial. Then the embryos are rinsed 3 to 5 times with PBST and the vial is placed on ice for 
15min. A monolayer of the embryos is glued to the center of a small petri dish using heptane glue and then covered with ice cold PBST. Then a very sharp tungsten needle is used to manually peel the embryos out of the vitellin membrane under a stereo microscope. The vitellin membranes stay glued to the bottom of the petri dish while the embryos can be transferred in PBST to a $1.5 \mathrm{ml}$ test tube for further processing.

\section{Maternal genotypes}

The genotypes of the analyzed embryos always refer to the maternal genotype, i.e. the genotype of the mother. For example, for analyzing homozygous lost mutants female virgins of the genotype $+/+;+/+; \operatorname{lost}[2] /$ lost [2] were crossed to wild type males. The embryos from that cross are then collected, fixed and analyzed as maternal lost mutant embryos.

\section{$\underline{\text { UAS-Gal4 system }}$}

The UAS-Gal4 system (Brand and Perrimon 1993) is used for the targeted expression of transgenes and other constructs. It is a binary system consisting of a fly line expressing the Gal4 transcription factor from yeast under the control of a specific promotor (the Gal4 driver line) and an effector fly line, which contains the desired construct under the control of an Gal4-specific promotor called UAS (upstream activating sequence) (the UAS line). Crossing these two fly lines yields offspring which have both, Gal4 expressed in specific tissue (depending on the driver line) and the UAS effector, which gets expressed only when Gal4 is present.

\section{$\underline{\text { Maternal RNAi }}$}

Many RNAs and proteins present in the early embryo are produced and deposited in the egg from the mother during oogenesis. Analyzing gene functions in early embryos therefore often require the use of maternal mutants to abolish the maternal contribution. 
Many mutations are homozygous lethal which makes it impossible to get adult mutant females. To address his problem the germ line clone (GLC) technique has been developed (Chou et al. 1993). Another, faster way of removing the maternal contribution of a gene is the use of RNAi in the adult female germ line (Staller et al. 2013).

The TRiP collection is a genome wide collection of transgenic fly strains which express a short hairpin RNA directed against a specific gene under the control of UAS (Ni et al. 2011). This allows knocking down gene functions using RNAi in a tissue specific manner using the Gal4-UAS system.

A fly bearing the desired UAS-shRNA construct (from the TRiP collection) is crossed to a Gal4 driver line which is active in the female germ line (MTD-Ga14). The female offspring from that cross, which expresses the shRNA in the germline, is crossed to wild type males. The resulting embryos are collected and analyzed.

\section{$\underline{\text { Fly crosses }}$}

For all analyses maternal genotypes are listed. That means that mothers of the listed genotype were crossed to wild type fathers. From these crosses the embryos were collected and analyzed. For imaging actin and myosin in live embryos w;UASp::UtrGFP,sqh::Sqh-Ch;MatGal4(15) was used as a control. This line crossed to ed RNAi yields $y v / w ; U A S p:: U t r-G F P, s q h:: S q h-C h / e d$ RNAi; MatGal4(15)/+ which express UtrGFP, Sqh-Ch and dsRNA against $e d$ in the female germ line. To obtain females which express Utr-GFP, Sqh-Ch and ed-nos-3'UTR in the germ line w;UASp::UtrGFP,sqh::Sqh-Ch;MatGal4(15) was crossed to w[*];UASp::ed-nos3'UTR yielding w[*];UASp::Utr-GFP,sqh::Sqh-Ch/UASp::ed-nos3'UTR;MatGal4(15)/+.

To obtain lost mutant females which express GFP-pgc-3'UTR in the germ line w; $P\{$ nos::nos-5'UTR-HA-GFP-HA-pgc-3'-UTR $\} / C y O ; M K R S / T M 6 B$ was crossed to $w ; ; l o s t[2] / T M 6 B \quad$ yielding $\quad w ; P\{$ nos::nos-5'UTR-HA-GFP-HA-pgc-3'-UTR\}/CyO; lost[2]/TM6B. From this stock females homozygous for lost [2] were selected. 
$\underline{\text { List of fly lines }}$

\begin{tabular}{|c|c|c|}
\hline fly line & genotype & source \\
\hline OregonR & wild type & \\
\hline lost[2] & +/+; +/+; lost[2]/TM6B float & (Graf 2007) \\
\hline lost RNAi & $\mathrm{y}[1] \mathrm{sc}[*] \mathrm{v}[1] ; \mathrm{P}\{\mathrm{y}[+\mathrm{t} 7.7] \mathrm{v}[+\mathrm{t} 1.8]=$ TRiP.GL01090 $\}$ attP2 & Bloomington \#38931 \\
\hline MTD-Gal4 & $\begin{array}{l}\mathrm{P}\{\mathrm{w}[+\mathrm{mC}]=\mathrm{otu}-\mathrm{GAL} 4:: \mathrm{VP} 16 . \mathrm{R}\} 1, \mathrm{w}[*] ; \mathrm{P}\{\mathrm{w}[+\mathrm{mC}]=\mathrm{GAL} 4- \\
\text { nos.NGT }\} 40 ; \mathrm{P}\{\mathrm{w}[+\mathrm{mC}]=\mathrm{GAL4}:: \mathrm{VP} 16- \\
\text { nos.UTR }\} \text { CG6325[MVD1] }\end{array}$ & Bloomington \#31777 \\
\hline lost $D f$ & w[1118]; Df(3R)BSC316/TM6B, Tb[+] & Bloomington \#24342 \\
\hline ed $R N A i$ & $\mathrm{y}[1] \mathrm{sc}[*] \mathrm{v}[1] ; \mathrm{P}\{\mathrm{y}[+\mathrm{t} 7.7] \mathrm{v}[+\mathrm{t} 1.8]=\mathrm{TRiP} . \mathrm{GL} 00648\} \mathrm{attP} 40$ & Bloomington \#38209 \\
\hline ed-nos-3'UTR & $\mathrm{w}\left[{ }^{*}\right] ; \mathrm{P}\{\mathrm{UASp}::$ ed-nos-3’UTR $\}$ & Vorbrüggen unpubl. \\
\hline lost GFP trap & $\mathrm{w}[1118] ; \mathrm{P}\{\mathrm{w}[+\mathrm{mC}]=\mathrm{PTT}-\mathrm{GA}\} \operatorname{lost}[\mathrm{ZCL} 3169]$ & Bloomington \#6832 \\
\hline $6 x[p g c]$ & $\mathrm{w}[*], \mathrm{P}\{\mathrm{w}[+], \mathrm{pgc}\} ;+/+; \mathrm{P}\{\mathrm{w}[+], \mathrm{pgc}\}$ & $\begin{array}{l}\text { (de Las Heras et al. } \\
\text { 2009) }\end{array}$ \\
\hline $3 x[\mathrm{pgc}] ; \mathrm{lost}$ & $\mathrm{P}\{\mathrm{w}[+], \mathrm{pgc}\} / \mathrm{FM} 7 ;$ lost$[2] / \mathrm{TM} 3$ & this study \\
\hline $\operatorname{pgc}[\Delta 1]$ & $\mathrm{w} ; \operatorname{pgc}[\Delta 1] / \mathrm{CyO}$ & $\begin{array}{l}\text { (Hanyu-Nakamura et } \\
\text { al. 2008) }\end{array}$ \\
\hline$p g c D f$ & $\operatorname{Dp}(1 ; \mathrm{Y}) \mathrm{y}[+] / \mathrm{y}[1] ; \mathrm{Df}(2 \mathrm{R}) \mathrm{X} 58-7, \operatorname{pr}[1] \mathrm{cn}[1] / \mathrm{CyO}, \mathrm{bw}[1]$ & Bloomington \#283 \\
\hline pgc RNAi & $\mathrm{y}[1] \mathrm{sc}[*] \mathrm{v}[1] ; \mathrm{P}\{\mathrm{y}[+\mathrm{t} 7.7] \mathrm{v}[+\mathrm{t} 1.8]=$ TRiP.HMS00601 $\}$ attP2 & Bloomington \#33720 \\
\hline$d h c 64 c$ & mwh[1] Dhc64C[6-10] h[1] st[1] p[p] e[s]/TM6B, Tb[1] & Bloomington \#8747 \\
\hline GFP-pgc-3'UTR & w; P\{nos::nos-5'UTR-HA-GFP-HA-pgc-3'-UTR $\} / C y O ;$ & (Rangan et al. 2009) \\
\hline UtrGFP;Sqh-Ch & w;UASp::Utr-GFP,sqh::Sqh-Ch;MatGal4(15) & (Rauzi et al. 2010) \\
\hline osk-nos-3'UTR & yw[67c23];P\{UASp::osk $\Delta$ 1,2-nos3'UTR $\}$ & (Little et al. 2015) \\
\hline
\end{tabular}

\section{Antibody staining}

Antibody staining was performed as described (Patel 1994) with some modifications. All of the following steps were carried out in $1.5 \mathrm{ml}$ test tubes at room temperature, if not stated otherwise. During all washing and incubation steps the samples were placed on a rotating wheel. When pipetting embryos cut-off pipette tips were used. The widened aperture of the tips prevents damaging the embryos via shearing forces.

The desired amount of fixed embryos is transferred together with some $\mathrm{MeOH}$ to a clean test tube and rinsed several times with $\mathrm{MeOH}$. The $\mathrm{MeOH}$ is then replaced by PBST in a stepwise manner. After blocking the sample with sheep serum in PBST the primary antibodies are added in the required dilution (see List of antibodies) and 
incubated over night at $4^{\circ} \mathrm{C}$. After washing and blocking appropriate secondary antibodies are added (and Dapi if needed) and incubated for 2 hours. This and all the following steps are carried out in darkness to prevent photobleaching. Afterwards the sample is thoroughly washed with PBST.

\section{Primary antibodies}

\begin{tabular}{|l|l|l|l|l|}
\hline Antigen & Host & Use & Dilution & Source \\
\hline Baz & $\begin{array}{l}\text { rabbit } \\
\text { polyclonal }\end{array}$ & $\begin{array}{l}\text { Bazooka, } \\
\text { adherens } \\
\text { junctions }\end{array}$ & $1: 1000$ & Wodarz et al. (1999) \\
\hline Digoxigenin & $\begin{array}{l}\text { sheep, Fab } \\
\text { fragments }\end{array}$ & $\begin{array}{l}\text { DIG-labeled } \\
\text { RNA probes, } \\
\text { HRP } \\
\text { conjugated }\end{array}$ & $1: 500$ & Roche cat. 1207733, Lot 87916922 \\
\hline $\begin{array}{l}\text { Dlg, clone } \\
\text { 4F3 }\end{array}$ & $\begin{array}{l}\text { mouse } \\
\text { IgG1 }\end{array}$ & $\begin{array}{l}\text { lateral } \\
\text { membranes }\end{array}$ & $1: 20$ & DSHB \\
\hline Ed & $\begin{array}{l}\text { rat } \\
\text { polyclonal }\end{array}$ & Echinoid & $1: 5000$ & Laplante and Nilson (2006) \\
\hline GFP & $\begin{array}{l}\text { chicken } \\
\text { polyclonal }\end{array}$ & GFP & $1: 1000$ & Abcam ab13970 \\
\hline Lost & $\begin{array}{l}\text { rabbit } \\
\text { polyclonal }\end{array}$ & Lost & $1: 200$ & Graf (2007) \\
\hline Osk & $\begin{array}{l}\text { rabbit } \\
\text { polyclonal }\end{array}$ & Oskar & $1: 2000$ & Anne Ephrussi \\
\hline Pgc & $\begin{array}{l}\text { rabbit } \\
\text { polyclonal }\end{array}$ & Pgc peptide & $1: 300$ & Hanyu-Nakamura et al. (2008) \\
\hline $\begin{array}{l}\text { p-Tyrosine, } \\
\text { clone PY20 }\end{array}$ & $\begin{array}{l}\text { mouse } \\
\text { IgG2b }\end{array}$ & cell borders & $1: 500$ & Biomol bml-sa240 \\
\hline $\begin{array}{l}\text { pSer2CTD, } \\
\text { clone H5 }\end{array}$ & $\begin{array}{l}\text { mouse } \\
\text { IgM }\end{array}$ & $\begin{array}{l}\text { transcriptional } \\
\text { activity }\end{array}$ & $1: 500$ & $\begin{array}{l}\text { Abcam ab24758 or BioLegend. Cat } \\
\text { 920202. Lot B200429 }\end{array}$ \\
\hline Slam & $\begin{array}{l}\text { guinea pig } \\
\text { polyclonal }\end{array}$ & furrow canal & $1: 5000$ & Jörg Großhans (Brandt 2006) \\
\hline Vasa & $\begin{array}{l}\text { rabbit } \\
\text { polyclonal }\end{array}$ & PGCs & $1: 5000$ & R.Jauch, unpublished \\
\hline Vasa & rat IgM \\
\hline $\begin{array}{l}\beta-T u b u l i n, ~ \\
\text { clone E7 }\end{array}$ & $\begin{array}{l}\text { mouse } \\
\text { IgG1 }\end{array}$ & PGCs & $1: 25$ & DSHB \\
\hline
\end{tabular}

\section{Secondary Antibodies}

Secondary antibodies were anti-rabbit, anti-mouse IgG, anti-rat, anti-chicken or antiguinea pig coupled to Alexa-488, Alexa-568 or Alexa-647. Anti-mouse IgM is coupled 
to DyLight-649. All secondary antibodies were purchased from Jackson ImmunoResearch and used in 1:500 dilution.

\section{$\underline{\text { FISH }}$}

Fluorescent in-situ hybridization was performed as described (Lecuyer 2011) with some modifications. The labeled antisense RNA probes were created in this study $(c y c B, p g c$, $g c l$ ) or a kind gift from Dr. Ralf Pflanz (nos, osk). All of the following steps were carried out in $1.5 \mathrm{ml}$ test tubes at room temperature, if not stated otherwise. During all washing and incubation steps the samples were placed on a rotating wheel. When pipetting embryos cut-off pipette tips were used. The widened aperture of the tips prevents damaging the embryos via shearing forces.

The desired amount of fixed embryos is transferred together with some $\mathrm{MeOH}$ to a clean test tube and rinsed several times with $\mathrm{MeOH}$. The $\mathrm{MeOH}$ is then replaced by PBST in a stepwise manner. The PBST is then replaced by Hybridazation buffer $(\mathrm{Hyb})$ in a stepwise manner. Afterwards the embryos are equilibrated in Hyb buffer in a $57^{\circ} \mathrm{C}$ waterbath for 1 hour, then the labeled antisense-RNA probe is added. The sample is then incubated overnight at $57^{\circ} \mathrm{C}$ in a waterbath.

After rinsing and washing the embryos with $57^{\circ} \mathrm{C} \mathrm{Hyb} \mathrm{buffer} \mathrm{the} \mathrm{Hyb} \mathrm{buffer} \mathrm{is} \mathrm{replaced}$ by PBST in a stepwise manner. The embryos are then incubated with an appropriate horseradish peroxidase (HRP) coupled antibody that detects the RNA probe (anti-DIG or anti-FITC). After washing off the unbound antibodies with PBST the embryos are equilibrated with the amplification diluent from the tyramide signal amplification (TSA) kit (PerkinElmer). Then the desired fluorophore solution from the TSA kit is added and incubated for 10 to 20min. After washing extensively with PBST the embryos can be mounted to a microscope slide or used for antibody staining.

\section{$\underline{\text { RISH }}$}

RNA in-situ hybridization (RISH) used to visualize ed RNA was performed as described (Tautz and Pfeifle 1989) with some modifications. The labeled antisense- 
RNA probe was obtained from Gerd Vorbrüggen (unpubl.). All of the following steps were carried out in $1.5 \mathrm{ml}$ test tubes at room temperature, if not stated otherwise. During all washing and incubation steps the samples were placed on a rotating wheel. When pipetting embryos cut-off pipette tips were used. The widened aperture of the tips prevents damaging the embryos via shearing forces.

The desired amount of fixed embryos is transferred together with some $\mathrm{MeOH}$ to a clean test tube and rinsed several times with $\mathrm{MeOH}$. The $\mathrm{MeOH}$ is then replaced by PBST in a stepwise manner. The PBST is then replaced by Hybridazation buffer (Hyb) in a stepwise manner. Afterwards the embryos are equilibrated in Hyb buffer in a $57^{\circ} \mathrm{C}$ waterbath for 1 hour, then the labeled antisense-RNA probe is added. The sample is then incubated overnight at $57^{\circ} \mathrm{C}$ in a waterbath.

After rinsing and washing the embryos with $57^{\circ} \mathrm{C} \mathrm{Hyb}$ buffer the Hyb buffer is replaced by PBST in a stepwise manner. The embryos are then incubated with an appropriate alkaline phosphatase (AP) coupled antibody that detects the RNA probe (anti-DIG or anti-FITC) at $4^{\circ} \mathrm{C}$ over night. After washing off the unbound antibodies with PBST the embryos are equilibrated in AP buffer solution and transferred to a white weighing dish. After adding NBT and BCIP and mixing the staining reaction can be observed using a stereo microscope. When the desired staining intensity is reached the reaction is stopped by adding PBST. After transferring the embryos to a $1.5 \mathrm{ml}$ test tube the sample is thoroughly rinsed and washed with PBST.

\section{Ovary preparation and staining}

Ovaries have been dissected from females expressing Lost-GFP (Lost GFP trap) in PBS. After rinsing the ovaries were incubated with phalloidin and Dapi for 30min, followed by washing with PBS. Then the ovaries were immediately put on a microscope slide in some PBS, spread out with forceps, covered with a coverslip and analyzed with a confocal microscope. 


\section{Imaging and analysis}

\section{Mounting stained embryos on a microscope slide}

\section{in VectaShield}

All mounting methods require stained embryos in PBST in a $1.5 \mathrm{ml}$ test tube.

After removing all excess of PBST from the test tube 2-3 drops of VectaShield (VectorLabs) are added and gently mixed. After the embryos have sunk to the bottom of the tube the excess of VectaShield is replaced by fresh VectaShield and gently mixed. The desired amount of embryos are transferred to a clean microscope slide using a cutoff pipette tip and covered with a coverslip without causing air bubbles. The edges are then sealed with nail polish.

\section{in DPX}

DPX is a non-aqueous mounting agent that provides good clearing while preventing bleaching (Espada et al. 2005). A variant which is less toxic than the classic recipe has been used (DPX new, Merck). The PBST in the test tube with the stained embryos is replaced by $100 \% \mathrm{EtOH}$ in a stepwise manner. After washing the sample with EtOH the EtOH is replaced by xylene (Sigma) in a stepwise manner. The desired amount of embryos is then transferred to a clean microscope slide using a cut-off pipette tip in as little xylene as possible. After removing the excess xylene with a tissue the embryos are quickly covered with DPX mounting medium followed by a coverslip. After 20-30min the edges are sealed with nail polish.

\section{in Canada balsam}

The PBST in the test tube with the stained embryos is replaced by $100 \% \mathrm{EtOH}$ in a stepwise manner. A drop of canada balsam is placed on a clean microscope slide. The desired amount of embryos is then transferred onto the canada balsam drop using a cut- 
off pipette tip in as little EtOH as possible. After drying the excess EtOH by gently blowing the embryos are stirred into the canada balsam and everything is covered with a coverslip. The canada balsam can take up to 24 hours to solidify.

\section{Confocal microscopy}

Confocal microscopy was performed using a LSM780 confocal laser scanning microscope (Zeiss). A Plan-Apochromat 25x/0.8 Imm Korr DIC or a Plan-Apochromat 40x/1.4 Oil DIC objective was used. Laser intensities and detector gain values were adjusted to prevent over exposure while using the full dynamic range.

\section{$\underline{\text { Image analysis and quantification }}$}

Confocal images were analyzed using Zen2011 (Zeiss) and Fiji, a variant of ImageJ (NIH). Figures were annotated and assembled using the ScientiFig plugin for Fiji (Aigouy and Mirouse 2013). The number of PGCs, transmigrating PGCs and transcriptionally active PGCs was counted manually using the CellCounter plugin for Fiji. 3D image stacks covering the complete posterior pole of an embryo were analyzed. PGCs were identified by the presence of Vasa. PGCs were counted as "transcriptionally active" when they showed a clear pCTD signal in their nucleus.

\section{Transcriptional activity in SNUPs}

The intensity of the nuclear pCTD signal was used as readout for transcriptional activity. Since the signal intensity is compared within each embryo (SNUPs vs. the other somatic nuclei) differences between stainings and imaging sessions can be ignored. For measuring the relative SNUP activity a custom macro for Fiji was created (see Appendix II for details). The macro batch-processes a defined input folder, which must 
contain the 3D confocal stacks to be analyzed. It requires .lsm files (from the LSM780 confocal microscope) oriented posterior up and stained for Vasa, pCTD and Dapi. After defining the input folder the macro opens the first stack and increases the brightness of each slice depending on its z-coordinate to compensate for the reduced signal intensity that occurs when recording 3D stacks. This step uses a custom made macro (see Appendix II for details). Then, after removing background the user is asked to set the measurement parameters which include threshold values for each channel which are saved a file. Then all open images are closed and the second stack is opened. After the input parameters for all files in the input folder have been set the macro starts the analysis.

First a mask is created from the Dapi channel, then a mask from Vasa channel. This requires the 3D Object Counter plugin for Fiji. Then, for each slice in the stack, the Vasa mask is subtracted from the Dapi mask, which now only contains the somatic nuclei. A rectangular selection created from the in y-direction extended bounding box of the Vasa mask is defined and applied to the Dapi mask. Everything of the Dapi mask inside this selection is treated as SNUP while everything outside is treated as a normal somatic nucleus. Using these masks and selections the signal intensity of the pCTD channel is measured only in the SNUPs and in the other somatic nuclei, respectively. This is done for each slice of the 3D stack. Then the cumulative pCTD signal intensity for the SNUPs and the rest of the soma are calculated and divided by the total number of analyzed voxels, which yields the average pCTD staining intensity. From these values the average pCTD signal intensity of the SNUPs relative to the other somatic nuclei is calculated.

This measurement procedure is used on every 3D stack in the input folder and all measured values are saved in a file. The results can then be subjected to statistical analysis.

\section{Germ plasm intensity profiles}

Here, the method is briefly described. For details refer to Appendix I: Protocols and to Appendix II: Fiji scripts. To visualize and quantify the distribution of germ plasm RNAs embryos are stained with an anti-Vasa antibody to label the germ plasm after the 
RNA of interest has been stained using FISH. Then 3D confocal stacks of posterior poles of stage 1 or 2 embryos are acquired covering the complete germ plasm.

For all stacks to be analyzed a y-projection is created using Fiji. In this projection the diameter of the germ plasm is measured using the "Line Selection" tool and the "Measure" function in Fiji.

For obtaining the intensity profiles a custom Fiji macro was created. It takes an input folder containing the .lsm files from the confocal microscope and creates y-projections for each stack. Then a 10 voxel high rectangular selection through the center of the germ plasm is created which spans the entire y-projection. On each x-position the mean intensity value within the selection is recorded using the "getProfile" function from Fiji and saved in a table. This is done for the Vasa channel as well as for the germ plasm FISH channel.

The intensity profiles are then smoothened and normalized. Then the profiles are scaled and adjusted in x-direction to the diameter and the position of the germ plasm in the $y$ projection. These calculations can be carried out in a spreadsheet. The data is imported into OriginPro 9, where the intensity profiles for a given staining and genotype are first averaged using the "Average Multiple Curves" tool and the averaged curve is plotted.

\section{Somatic vs. PGC distribution of $o s k$ and $p g c$ RNA}

Stage 4 embryos are analyzed. Because of some Vasa protein left in the soma after PGC budding it is not possible to use a mask from the Vasa channel to distinguish between a somatic RNA signal and an RNA signal which is within the PGCs. Instead the identity of the nuclei (somatic or PGC nucleus) is used to determine whether a FISH signal is somatic or with a PGC. This requires 3D confocal stacks covering the complete posterior pole of an embryo stained with Vasa, Dapi and FISH of the RNA of interest. For that, a series of custom Fiji macros was created (see Appendix II: Fiji macros for details) which use several functions of the ImageJ 3D Suite plugin (Ollion et al. 2013). All macros are batch-processing all files from a defined input folder. The first macro takes an input folder containing the .lsm files from the confocal microscope and splits the channels. Then it calls a second macro which identifies all nuclei as single objects in 3D from the Dapi channel. This is done by first finding local maxima of signal intensity 
using the "3D Fast Filters" tool from the 3D Suite plugin. The maxima are then used as seeds for the 3D watershed algorithm from the 3D Suite plugin. This yields a list of 3D objects representing all nuclei in the image stack. Then around the centroid of each nucleus a sphere with a radius of $5 \mu \mathrm{m}$ is drawn. This yields a list of spheres each representing a volume around a specific nucleus. This list is saved to a file.

The first macro then calls a third macro which uses the Vasa channel and the list of spheres as inputs. The macro then measures the average Vasa staining intensity within each of the spheres. The spheres with a high Vasa intensity are considered to represent PGC nuclei, while the others represent somatic nuclei. The macro then labels each object in the list according to its identity and saves the labeled list to a file. The assignment of the nuclear identities is manually checked and corrected if needed. A list of objects which contains only spheres around somatic nuclei is saved to a file.

Then a forth macro uses this list to define the regions for measuring the FISH intensities. For this, a mask is created from the Vasa channel of a given embryo. This mask contains the PGCs as well as the germ plasm left in the soma. Since the somatic germ plasm always localizes directly apical to the somatic nuclei, these regions can be removed from the Vasa mask by deleting everything within a sphere around each somatic nucleus. These spheres are defined by the list of objects obtained from the third macro. After that the Vasa mask only contains the PGCs without the somatic remnants of the germ plasm. Using the mask the signal intensity of the FISH channel is measured within the mask and then outside the mask. Then the cumulative FISH signal intensity for the PGCs and the soma are calculated and divided by the total number of analyzed voxels, which yields the average FISH staining intensity representing the amount of RNA. From these values the relative amount of osk RNA within the PGCs or the relative amount of $p g c$ RNA in the soma can be inferred.

This measurement procedure is used on every $3 \mathrm{D}$ stack in the input folder and all measured values are saved in a file. The results can then be subjected to statistical analysis. 


\section{Statistics and data visualization}

Statistical significance of differences between groups was tested using Mann-Whitney$\mathrm{U}$ test. All calculations if not stated otherwise have been performed in OriginPro 9 (OriginLabs). Data was visualized using OriginPro 9 (OriginLabs).

For calculating the statistical significance of the difference between the $p g c$ FISH intensity profiles of wild type and lost embryos the adjusted and normalized profile curves have been linearly interpolated using the "Interpolate" function in OriginPro9. This generated a set of interpolated data with 500 evenly spaced $\mathrm{x}$-values. Then for each of the 500 points on the $\mathrm{x}$-axis a Mann-Whitney-U test was calculated to obtain a set of 500 p-values which can be plotted against the interpolated x-values. In the center of the germ plasm, where the $p g c$ FISH intensity profile in lost embryos differs from wild type, the p-values are below 0.0001 .

\section{Molecular biology}

\section{Extraction of genomic DNA from flies}

Genomic DNA which can be used as a template for PCR is prepared as described (Gloor et al. 1993) with minor modifications. In brief a single anesthetized fly is put into a $1.5 \mathrm{ml}$ test tube. The fly is then mashed in Squishing buffer (SB) with a pipette tip and incubated for $30 \mathrm{~min}$ at room temperature. Then the tube is heated to $95^{\circ} \mathrm{C}$ to inactivate the Proteinase $\mathrm{K}$ from the SB. After a brief centrifugation the supernatant can be used as a template for PCR or stored in the fridge for several weeks.

\section{Template vector for creating in-situ probes}

A specific labeled antisense RNA probe is needed to visualize RNA using (fluorescent) in-situ hybridization. The probes are produced via in-vitro transcription from a suitable 
template vector, which contains a fragment of the gene of interest in antisense direction downstream of a universal promotor. The template vector can be created using standard molecular cloning techniques.

First genomic DNA from wild type flies is prepared (see method "Extraction of genomic DNA from flies"). Then the desired fragment is amplified using the genomic DNA as a template for PCR using Phusion High-Fidelity DNA polymerase (NEB) according the manufacturer's instructions. The primers are listed below.

The success of the PCR is verified by agarose gel electrophoresis and the fragment is extracted from the gel using a Gel extraction kit (Qiagen) according to the manufacturer's instructions. The purified fragment is then cloned into the pJet1.2-blunt vector using the CloneJet PCR Cloning Kit (Thermo Fisher Scientific) according to the manufacturer's instructions and transformed into competent bacteria (One Shot TOP10. Invitrogen) according to the manufacturer's instructions. Positive clones are identified via colony PCR according to the protocol provided with the CloneJet PCR Cloning Kit (Thermo Fisher Scientific) using the pJet1.2-forward sequencing primer and the FORWARD primer from the genomic PCR. This ensures that the fragment is inserted in the correct orientation for producing antisense RNA. A positive clone is chosen and the vector amplified via a midi scale plasmid preparation (see method "Medium scale plasmid preparation"). An aliquot of the template vector is then sent for sequencing to MWG Operon (Eurofins Genomics) to verify the sequence and the orientation of the insert.

\section{$\underline{\text { Gel electrophoresis }}$}

For size separation and purification of DNA fragment agarose gel electrophoresis was used. Agarose $(1 \% \mathrm{w} / \mathrm{w})$ was dissolved TAE buffer, boiled, mixed with ethidium bromide and casted into gel chambers. DNA fragments were visualized using a UV lamp. 


\section{DNA oligos (Primers) used for PCR}

Primers were designed using PerlPrimer (v1.1.21) and produced by MWG (Eurofins Genomics). The following DNA oligos were used:

\begin{tabular}{|l|l|l|}
\hline Name & Sequence 5' $\rightarrow \mathbf{3}$ & purpose \\
\hline pJet1.2-forward & CGACTCACTATAGGGAGAGCGGC & Colony-PCR, squencing \\
\hline cycB_ex4_for & GATGAAGTACTTGGACATCGT & $\begin{array}{l}\text { PCR for creating template for } \\
\text { creating } c y c B \text { in-situ probe }\end{array}$ \\
\cline { 1 - 2 } cycB_ex4_rev & TACAGTCAAGTCCAGTTCTTCC & $\begin{array}{l}\text { PCR for creating template for } \\
\text { creating } g c l \text { in-situ } \text { probe }\end{array}$ \\
\cline { 1 - 2 } gcl_ex3_for & TGATCCGGCCCAGTTAGACACC &
\end{tabular}

\section{Medium scale plasmid preparation}

A 45ml bacterial culture from a single colony was grown overnight. Plasmid preparation was performed using PlasmidPlus Midi Kit (Qiagen) according to the manufacturer's instructions. DNA was eluted in $200 \mu l$ water and the concentration was measured using a spectrophotometer (NanoDrop, Thermo Scientific).

\section{Creating labeled antisense RNA in-situ probes}

Labeled antisense RNA probes are produced via in-vitro transcription from a suitable template vector. Antisense RNA against $p g c$ was created from a cDNA clonse (RE14873, Drosophila Genome Resource Center). Probes against $c y c B$ and $g c l$ were created from a genomic clone (see method "Template vector for creating in-situ probes"). First the template is linearized using a restriction endonuclease which cuts downstream of the insert without disrupting the promoter-insert cassette. The linearized plasmid is then purified using Qiaquick Nucleotide Removal Kit (Qiagen) according to the manufacturer's instructions. The labeled antisense RNA probe is produced using the DIG RNA Labeling Kit (Roche) according to the manufacturer's instructions. The 
reaction contains the linearized plasmid as a template, NTP labeling mix, a suitable RNA polymerase, reaction buffer and RNAse inhibitor. After the incubation the success of the reaction is verified by loading a small aliquot of the reaction to an agarose gel for electrophoresis. The labeled RNA probe is then purified by EtOH precipitation. In brief, $\mathrm{EtOH}$, water and sodium acetate are added to the reaction which causes the RNA to precipitate. After centrifugation the supernatant is discarded and the RNA pellet washed with EtOH. Afterwards the dried pellet is resuspended in Hybe buffer and stored at $20^{\circ} \mathrm{C}$.

\section{Materials}

Fix solution $\quad 4 \%$ paraformaldehyde in PBS, 20 mM EGTA

heptane glue Fixogum (Marabu) 1:15 in heptane

Hyb buffer $\quad 50 \%$ formamide, $5 x$ SSC, $200 \mu \mathrm{g} / \mathrm{ml}$ ssDNA, $100 \mu \mathrm{g} / \mathrm{ml}$ tRNA, $50 \mu \mathrm{g} / \mathrm{ml}$ heparin

PBS $\quad 1.37 \mathrm{M} \mathrm{NaCl}, 100 \mathrm{mM} \mathrm{Na} 2 \mathrm{HPO} 4,27 \mathrm{mM} \mathrm{KCl}, 20 \mathrm{mM}$ KH2HPO4, pH 7.4

PBTS PBS $+0.1 \%(\mathrm{v} / \mathrm{v})$ Triton $\mathrm{X}-100$

Squishing buffer $10 \mathrm{mM}$ Tris- $\mathrm{HCl}$ (pH 8.2); $1 \mathrm{mM}$ EDTA, $25 \mathrm{mM} \mathrm{NaCl}, 200 \mu \mathrm{g} / \mathrm{ml}$ Proteinase K (freshly added)

TAE (50X) $\quad 2 \mathrm{M}$ Tris, $1 \mathrm{M}$ acetic acid, $50 \mathrm{mM}$ EDTA, pH 8.4

\section{Echinoid project contributions}

The echinoid project was started as a lab rotation and bachelor thesis project by Ninett Wolfram under the practical supervision by the author of this dissertation. The following results were obtained by Ninett Wolfram and reported in her Bachelor thesis:

- $\quad$ ed RISH on wild type embryos (Fig. 4 A)

- fly crosses to obtain $e d[F 72]$ GLC mutant embryos 
- antibody staining ( $\alpha$-Vasa, $\alpha$-pTyr) on wild type and ed[F72] GLC embryos (Fig. 5 ABDEGH)

- quantifying transmigrating PGCs in wild type and ed[F72] GLC embryos (Fig. $5 \mathrm{~J}$, first two columns)

- molecular cloning to create a pUASp-ed-nos3'UTR vector

- establishing transgenic fly lines containing UASp-ed-nos3'UTR construct

- fly crosses to obtain embryos with maternal ed-nos-3'UTR contribution

- ed RISH on ed-nos3'UTR embryos (Fig. 6 A,B)

- quantifying transmigrating PGCs in ed-nos3'UTR embryos (Fig. $6 \mathrm{~J}$ )

All results from the echinoid project not mentioned in the above list were obtained by the author of this dissertation. 



\section{List of abbreviations}

\begin{tabular}{|c|c|}
\hline AEL & after egg laying \\
\hline AJ & adherens junction \\
\hline AP & alkaline phosphatase \\
\hline BCIP & 5-Bromo-4-chloro-3-indolyl phosphate \\
\hline CNS & central nervous system \\
\hline CTD & carboxyterminal domain \\
\hline DIG & Digoxigenin \\
\hline DSHB & Developmental Studies Hybridoma Bank \\
\hline EMT & epithelial-to-mesenchymal transition \\
\hline $\mathrm{EtOH}$ & ethanol \\
\hline $\mathrm{FC}$ & furrow canal \\
\hline FISH & fluorescent in-situ hybridization \\
\hline GBE & germ band extension \\
\hline GFP & green fluorescent protein \\
\hline GLC & germline clone \\
\hline GOF & gain-of-function \\
\hline GSC & germline stem cell \\
\hline HRP & horseradish peroxidase \\
\hline LOF & loss-of-function \\
\hline $\mathrm{MeOH}$ & methanol \\
\hline MZT & maternal-to-zygotic transition \\
\hline NBT & nitroblue tetrazolium \\
\hline ORF & open reading frame \\
\hline PBS & phosphate-buffered saline \\
\hline PBST & phosphate-buffered saline + Triton $X-100$ \\
\hline PCR & polymerase chain reaction \\
\hline pCTD & phosphorylated CTD \\
\hline PFA & para-formaldehyde \\
\hline PGCs & primordial germ cells \\
\hline PMG & posterior midgut \\
\hline RNA & ribonucleic acid \\
\hline
\end{tabular}


RNP

RRM

SB

SEM

SGP

SNUPs

TAE

TSA

UAS

UTR ribonucleoprotein particle

RNA recognition motif

squishing buffer

standard error of the mean

somatic gonadal precursor

somatic nuclei underneath the PGCs

Tris acetate EDTA buffer

tyramide signal amplification

upstream activating sequence

untranslated region 


\section{List of genes}

\begin{tabular}{|c|c|}
\hline Abbreviation & Gene name \\
\hline$a P K C$ & atypical protein kinase $C$ \\
\hline arm & armadillo, $\beta$-Catenin \\
\hline Arp-1 & Actin-related protein 1 \\
\hline$a u b$ & aubergine \\
\hline$b a z$ & bazooka \\
\hline$b c d$ & bicoid \\
\hline bru & bruno \\
\hline$c l b$ & columbus, HMG Coenzyme A reductase \\
\hline cno & canoe \\
\hline$c r b$ & crumbs \\
\hline cup & cup \\
\hline$C y c B$ & Cyclin B \\
\hline Dhc64C & Dynein heavy chain $64 C$ \\
\hline$d l g$ & discs-large \\
\hline$e d$ & echinoid \\
\hline foi & fear-of-intimacy \\
\hline gcl & germ cell-less \\
\hline Gir & Girdin \\
\hline grk & gurken \\
\hline$h k b$ & huckebein \\
\hline $\lg l$ & lethal (2) giant larvae \\
\hline lost & lost \\
\hline mle & maleless \\
\hline nos & nanos \\
\hline orb & ool8 RNA-binding protein \\
\hline osk & oskar \\
\hline$p A b p$ & poly $(A)$ binding protein \\
\hline$p g c$ & polar granule component \\
\hline Rhol & Rhol \\
\hline
\end{tabular}

CG number

CG42783

CG11579

CG6174

CG6137

CG5055

CG1034

CG31762

CG10367

CG42312

CG6383

CG11181

CG3510

CG7507

CG1725

CG12676

CG6817

CG8411

CG12734

CG17610

CG9768

CG2671

CG14648

CG11680

CG5637

CG10868

CG10901

CG5119

CG32885

CG8416 


$\begin{array}{lll}\text { RhoGEF2 } & \text { Rho guanine nucleotide exchange factor 2 } & \text { CG9635 } \\ \text { rump } & \text { rumpelstiltskin } & \text { CG9373 } \\ \text { scrib } & \text { scribbled } & \text { CG43398 } \\ \text { sdt } & \text { stardust } & \text { CG32717 } \\ \text { shg } & \text { shotgun, DE-Cadherin } & \text { CG3722 } \\ \text { slam } & \text { slow as molasses } & \text { CG9506 } \\ \text { srp } & \text { serpent } & \text { CG3992 } \\ \text { stau } & \text { staufen } & \text { CG5753 } \\ \text { tral } & \text { trailer hitch } & \text { CG10686 } \\ \text { Tre1 } & \text { Trapped in endoderm-1 } & \text { CG3171 } \\ \text { tud } & \text { tudor } & \text { CG9450 } \\ \text { vas } & \text { vasa } & \text { CG46283 } \\ \text { wun } & \text { wunen } & \text { CG8804 } \\ \text { wun2 } & \text { wunen-2 } & \text { CG8805 } \\ \text { Zn72D } & \text { zinc-finger protein at } 72 D & \text { CG5215 } \\ \alpha-C a t & \alpha-C a t e n i n & \text { CG17947 }\end{array}$

Earlier the gene lost (GC14648) was called aeneas (aen) internally and can be found under that name in Molitor (2002) and Graf (2007). 


\section{List of figures}

Figure Page

Fig. 1: The germ plasm is deposited at the posterior pole during oogenesis $\quad 7$

Fig. 2: Cell polarity and adhesion are established during cellularization. $\quad 10$

Fig. 3: Schematic representation of the migration of the PGCs. 13

$\begin{array}{ll}\text { Fig. 4: Ed is found the embryonic epithelium but not in the PGCs. } & 21\end{array}$

Fig. 5: In embryos which lack maternal Ed the PGCs mix with the underlying epithelium. 23

Fig. 6: Ectopic expression of $e d$ within the PGCs leads to PGCs mixing with the epithelium. 25

Fig. 7: Ed is removed from the PMG cells when the PGCs transmigrate. 27

Fig. 8: Cellularization is not disturbed in $e d$ RNAi and $e d$-nos-UTR embryos. 28

Fig. 9: The apical actin network is disrupted in fixed ed RNAi and ed-nos-UTR embryos. 29

Fig. 10: The apical actin network is disrupted in live ed RNAi and ed-nos-UTR embryos. 30

Fig. 11: Lost is a sponge body component and ubiquitously present in early embryos. 33

Fig. 12: The PGCs in embryos which lack maternal lost cross the PMG epithelium prematurely. $\quad 35$

Fig. 13: The PGCs in embryos which lack maternal lost show ectopic transcriptional activity 36

Fig. 14: Cellularization phenotypes in lost embryos are restricted to the very posterior pole. 39

Fig. 15: The somatic nuclei underneath the PGCs (SNUPs) have reduced levels of transcription in embryos which lack maternal lost. $\quad 42$

Fig. 16: The localization patterns of germ plasm RNAs revealed by FISH. 44

Fig. 17: The localization patterns of most germ plasm RNAs don't differ between wild type and lost. $\quad 46$

Fig. 18: The average relative pgc FISH staining intensity in the center of the germ plasm is higher in maternal lost embryos. $\quad 48$

Fig. 19: lost interacts genetically with $p g c$ GOF. $\quad 50$

Fig. 20: The PGCs in embryos which lack maternal lost or maternal $p g c$ show ectopic transcriptional activity.

Fig. 21: lost interacts genetically with dynein heavy chain (dhc).

Fig. 22: The amount of $p g c$ RNA which remains in the soma after PGC formation is not increased in maternal lost mutants.

Fig. 23: Maternal loss of lost does not drastically affect the localization of Pgc peptide or a $p g c$ translation reporter construct after PGC formation.

Fig. 24: Embryos from mothers expressing GFP-pgc-3'UTR show lost-like phenotypes. 59

Fig. 25: The amount of osk RNA within the PGCs is drastically increased in maternal lost mutants. $\quad 61$

Fig. 26: The localization of osk RNA is highly dynamic during PGC formation. 64

Fig. 27: The localization of Osk protein is not affected in maternal lost and in osk-nos-3'UTR embryos $\quad 66$

Fig. 28: Maternal expression of osk-nos-3'UTR is sufficient to cause lost-like phenotypes. 69 



\section{Declaration}

I hereby declare that I wrote this dissertation "The early Development of the Germline in Drosophila melanogaster" on my own and without the use of any other than the cited sources and aids.

Place, Date

Signature 



\section{References}

Acharya, S., P. Laupsien, C. Wenzl, S. Yan and J. Grosshans (2013). "Function and dynamics of slam in furrow formation in early Drosophila embryo." Dev Biol.

Aigouy, B. and V. Mirouse (2013). "ScientiFig: a tool to build publication-ready scientific figures." Nat Methods 10(11): 1048.

Allan, V. (1996). "Motor proteins: a dynamic duo." Current biology : CB 6(6): 630-633.

Anne, J. (2010). "Targeting and anchoring Tudor in the pole plasm of the Drosophila oocyte." PLoS One 5(12): e14362.

Arkov, A. L., J. Y. Wang, A. Ramos and R. Lehmann (2006). "The role of Tudor domains in germline development and polar granule architecture." Development 133(20): 4053-4062.

Bai, J., W. Chiu, J. Wang, T. Tzeng, N. Perrimon and J. Hsu (2001). "The cell adhesion molecule Echinoid defines a new pathway that antagonizes the Drosophila EGF receptor signaling pathway." Development 128(4): 591-601.

Baltz, A. G., M. Munschauer, B. Schwanhausser, A. Vasile, Y. Murakawa, M. Schueler, N. Youngs, D. Penfold-Brown, K. Drew, M. Milek, E. Wyler, R. Bonneau, M. Selbach, C. Dieterich and M. Landthaler (2012). "The mRNA-bound proteome and its global occupancy profile on protein-coding transcripts." Mol Cell 46(5): 674-690.

Bastock, R. and D. S. Johnston (2008). "Drosophila oogenesis." Current biology : CB 18(23): R1082-1087.

Bate, M. and A. Martinez-Arias, Eds. (1993). The Development of Drosophila melanogaster. New York, Cold Spring Harbor Laboratory Press.

Bilder, D., M. Li and N. Perrimon (2000). "Cooperative regulation of cell polarity and growth by Drosophila tumor suppressors." Science 289(5476): 113-116.

Bilder, D. and N. Perrimon (2000). "Localization of apical epithelial determinants by the basolateral PDZ protein Scribble." Nature 403(6770): 676-680.

Boyle, M. and S. DiNardo (1995). "Specification, migration and assembly of the somatic cells of the Drosophila gonad." Development 121(6): 1815-1825.

Brand, A. H. and N. Perrimon (1993). "Targeted gene expression as a means of altering cell fates and generating dominant phenotypes." Development 118(2): 401-415. 
Brandt, A., F. Papagiannouli, N. Wagner, M. Wilsch-Brauninger, M. Braun, E. E. Furlong, S. Loserth, C. Wenzl, F. Pilot, N. Vogt, T. Lecuit, G. Krohne and J. Grosshans (2006). "Developmental control of nuclear size and shape by Kugelkern and Kurzkern." Curr Biol 16(6): 543-552.

Breitwieser, W., F. H. Markussen, H. Horstmann and A. Ephrussi (1996). "Oskar protein interaction with Vasa represents an essential step in polar granule assembly." Genes Dev 10(17): 2179-2188.

Campbell, K., G. Whissell, X. Franch-Marro, E. Batlle and J. Casanova (2011). "Specific GATA factors act as conserved inducers of an endodermal-EMT." Dev Cell 21(6): 1051-1061.

Castagnetti, S. and A. Ephrussi (2003). "Orb and a long poly(A) tail are required for efficient oskar translation at the posterior pole of the Drosophila oocyte." Development 130(5): $835-843$.

Castello, A., B. Fischer, K. Eichelbaum, R. Horos, B. M. Beckmann, C. Strein, N. E. Davey, D. T. Humphreys, T. Preiss, L. M. Steinmetz, J. Krijgsveld and M. W. Hentze (2012). "Insights into RNA biology from an atlas of mammalian mRNA-binding proteins." Cell 149(6): 1393-1406.

Chou, T. B., E. Noll and N. Perrimon (1993). "Autosomal P[ovoD1] dominant femalesterile insertions in Drosophila and their use in generating germ-line chimeras." Development 119(4): 1359-1369.

Coravos, J. S. and A. C. Martin (2016). "Apical Sarcomere-like Actomyosin Contracts Nonmuscle Drosophila Epithelial Cells." Dev Cell.

da Silva, S. M. and J. P. Vincent (2007). "Oriented cell divisions in the extending germband of Drosophila." Development 134(17): 3049-3054.

Dahlgaard, K., A. A. Raposo, T. Niccoli and D. St Johnston (2007). "Capu and Spire assemble a cytoplasmic actin mesh that maintains microtubule organization in the Drosophila oocyte." Dev Cell 13(4): 539-553.

Dahmus, M. E. (1996). "Reversible phosphorylation of the C-terminal domain of RNA polymerase II." J Biol Chem 271(32): 19009-19012.

de Las Heras, J. M., R. G. Martinho, R. Lehmann and J. Casanova (2009). "A functional antagonism between the pgc germline repressor and torso in the development of somatic cells." EMBO reports 10(9): 1059-1065.

DeFalco, T. J., G. Verney, A. B. Jenkins, J. M. McCaffery, S. Russell and M. Van Doren (2003). "Sex-specific apoptosis regulates sexual dimorphism in the Drosophila embryonic gonad." Developmental cell 5(2): 205-216. 
Desai, R., R. Sarpal, N. Ishiyama, M. Pellikka, M. Ikura and U. Tepass (2013). "Monomeric alpha-catenin links cadherin to the actin cytoskeleton." Nat Cell Biol 15(3): 261-273.

Dienstbier, M., F. Boehl, X. Li and S. L. Bullock (2009). "Egalitarian is a selective RNA-binding protein linking mRNA localization signals to the dynein motor." Genes \& development 23(13): 1546-1558.

Duque, J. and N. Gorfinkiel (2016). "Integration of actomyosin contractility with cellcell adhesion during dorsal closure." Development 143(24): 4676-4686.

Edgar, B. A., C. P. Kiehle and G. Schubiger (1986). "Cell cycle control by the nucleocytoplasmic ratio in early Drosophila development." Cell 44(2): 365-372.

Ephrussi, A., L. K. Dickinson and R. Lehmann (1991). "Oskar organizes the germ plasm and directs localization of the posterior determinant nanos." Cell 66(1): 37-50.

Escudero, L. M., S. Y. Wei, W. H. Chiu, J. Modolell and J. C. Hsu (2003). "Echinoid synergizes with the Notch signaling pathway in Drosophila mesothorax bristle patterning." Development 130(25): 6305-6316.

Espada, J., A. Juarranz, S. Galaz, M. Canete, A. Villanueva, M. Pacheco and J. C. Stockert (2005). "Non-aqueous permanent mounting for immunofluorescence microscopy." Histochem Cell Biol 123(3): 329-334.

Forrest, K. M. and E. R. Gavis (2003). "Live imaging of endogenous RNA reveals a diffusion and entrapment mechanism for nanos mRNA localization in Drosophila." Curr Biol 13(14): 1159-1168.

Geer, L. Y., M. Domrachev, D. J. Lipman and S. H. Bryant (2002). "CDART: protein homology by domain architecture." Genome Res 12(10): 1619-1623.

Gelbart, W. M. and D. B. Emmert (2010). FlyBase High Throughput Expression Pattern Data Beta Version.

Giot, L., J. S. Bader, C. Brouwer, A. Chaudhuri, B. Kuang, Y. Li, Y. L. Hao, C. E. Ooi, B. Godwin, E. Vitols, G. Vijayadamodar, P. Pochart, H. Machineni, M. Welsh, Y. Kong, B. Zerhusen, R. Malcolm, Z. Varrone, A. Collis, M. Minto, S. Burgess, L. McDaniel, E. Stimpson, F. Spriggs, J. Williams, K. Neurath, N. Ioime, M. Agee, E. Voss, K. Furtak, R. Renzulli, N. Aanensen, S. Carrolla, E. Bickelhaupt, Y. Lazovatsky, A. DaSilva, J. Zhong, C. A. Stanyon, R. L. Finley, Jr., K. P. White, M. Braverman, T. Jarvie, S. Gold, M. Leach, J. Knight, R. A. Shimkets, M. P. McKenna, J. Chant and J. M. Rothberg (2003). "A protein interaction map of Drosophila melanogaster." Science 302(5651): $1727-1736$.

Gloor, G. B., C. R. Preston, D. M. Johnson-Schlitz, N. A. Nassif, R. W. Phillis, W. K. Benz, H. M. Robertson and W. R. Engels (1993). "Type I repressors of P element mobility." Genetics 135(1): 81-95. 
Goldstein, J. L. and M. S. Brown (1990). "Regulation of the mevalonate pathway." Nature 343(6257): 425-430.

Graf, R. (2007). Characterization of the Role of aeneas in Primordial Germ Cell Migration and Blastoderm Cellularization. Dr. rer. nat. Dissertation, Georg-AugustUniversität Göttingen.

Hanyu-Nakamura, K., S. Kobayashi and A. Nakamura (2004). "Germ cell-autonomous Wunen2 is required for germline development in Drosophila embryos." Development 131(18): 4545-4553.

Hanyu-Nakamura, K., H. Sonobe-Nojima, A. Tanigawa, P. Lasko and A. Nakamura (2008). "Drosophila Pgc protein inhibits P-TEFb recruitment to chromatin in primordial germ cells." Nature 451(7179): 730-733.

Harris, T. J. and M. Peifer (2004). "Adherens junction-dependent and -independent steps in the establishment of epithelial cell polarity in Drosophila." J Cell Biol 167(1): 135-147.

Harris, T. J. and M. Peifer (2005). "The positioning and segregation of apical cues during epithelial polarity establishment in Drosophila." J Cell Biol 170(5): 813-823.

Herold, N., C. L. Will, E. Wolf, B. Kastner, H. Urlaub and R. Luhrmann (2009). "Conservation of the protein composition and electron microscopy structure of Drosophila melanogaster and human spliceosomal complexes." Molecular and cellular biology 29(1): 281-301.

Hertel, A. (2011). Migration von primordialen Keimbahnstammzellen in Drosophila melanogaster. Dipl.-Biol. Diploma Thesis, Georg-August-Universität Göttingen.

Houssin, E., U. Tepass and P. Laprise (2015). "Girdin-mediated interactions between cadherin and the actin cytoskeleton are required for epithelial morphogenesis in Drosophila." Development 142(10): 1777-1784.

Irvine, K. D. and E. Wieschaus (1994). "Cell intercalation during Drosophila germband extension and its regulation by pair-rule segmentation genes." Development 120(4): 827-841.

Jaglarz, M. K. and K. R. Howard (1994). "Primordial germ cell migration in Drosophila melanogaster is controlled by somatic tissue." Development 120(1): 83-89.

Jaglarz, M. K. and K. R. Howard (1995). "The active migration of Drosophila primordial germ cells." Development 121(11): 3495-3503.

Jain, R. A. and E. R. Gavis (2008). "The Drosophila hnRNP M homolog Rumpelstiltskin regulates nanos mRNA localization." Development 135(5): 973-982. 
Jambor, H., C. Brunel and A. Ephrussi (2011). "Dimerization of oskar 3' UTRs promotes hitchhiking for RNA localization in the Drosophila oocyte." RNA 17(12): 2049-2057.

Jambor, H., S. Mueller, S. L. Bullock and A. Ephrussi (2014). "A stem-loop structure directs oskar mRNA to microtubule minus ends." RNA 20(4): 429-439.

Jenkins, A. B., J. M. McCaffery and M. Van Doren (2003). "Drosophila E-cadherin is essential for proper germ cell-soma interaction during gonad morphogenesis." Development 130(18): 4417-4426.

Jenny, A., O. Hachet, P. Zavorszky, A. Cyrklaff, M. D. Weston, D. S. Johnston, M. Erdelyi and A. Ephrussi (2006). "A translation-independent role of oskar RNA in early Drosophila oogenesis." Development 133(15): 2827-2833.

Kanke, M., H. Jambor, J. Reich, B. Marches, R. Gstir, Y. H. Ryu, A. Ephrussi and P. M. Macdonald (2015). "oskar RNA plays multiple noncoding roles to support oogenesis and maintain integrity of the germline/soma distinction." RNA 21(6): 1096-1109.

Kanke, M. and P. M. Macdonald (2015). "Translational activation of oskar mRNA: reevaluation of the role and importance of a 5' regulatory element [corrected]." PLoS One 10(5): e0125849.

Karr, T. L. and B. M. Alberts (1986). "Organization of the cytoskeleton in early Drosophila embryos." The Journal of cell biology 102(4): 1494-1509.

Khuc Trong, P., H. Doerflinger, J. Dunkel, D. St Johnston and R. E. Goldstein (2015). "Cortical microtubule nucleation can organise the cytoskeleton of Drosophila oocytes to define the anteroposterior axis." Elife 4.

Kim-Ha, J., K. Kerr and P. M. Macdonald (1995). "Translational regulation of oskar mRNA by bruno, an ovarian RNA-binding protein, is essential." Cell 81(3): 403-412.

Krieg, M., Y. Arboleda-Estudillo, P. H. Puech, J. Kafer, F. Graner, D. J. Muller and C. P. Heisenberg (2008). "Tensile forces govern germ-layer organization in zebrafish." Nat Cell Biol 10(4): 429-436.

Kumar, S., C. Konikoff, B. Van Emden, C. Busick, K. T. Davis, S. Ji, L. W. Wu, H. Ramos, T. Brody, S. Panchanathan, J. Ye, T. L. Karr, K. Gerold, M. McCutchan and S. J. Newfeld (2011). "FlyExpress: visual mining of spatiotemporal patterns for genes and publications in Drosophila embryogenesis." Bioinformatics 27(23): 3319-3320.

Kunwar, P. S., H. Sano, A. D. Renault, V. Barbosa, N. Fuse and R. Lehmann (2008). "Tre1 GPCR initiates germ cell transepithelial migration by regulating Drosophila melanogaster E-cadherin." The Journal of cell biology 183(1): 157-168. 
Kunwar, P. S., M. Starz-Gaiano, R. J. Bainton, U. Heberlein and R. Lehmann (2003). "Tre1, a G protein-coupled receptor, directs transepithelial migration of Drosophila germ cells." PLoS biology 1(3): E80.

Laplante, C. and L. A. Nilson (2006). "Differential expression of the adhesion molecule Echinoid drives epithelial morphogenesis in Drosophila." Development 133(16): 32553264.

Laplante, C. and L. A. Nilson (2011). "Asymmetric distribution of Echinoid defines the epidermal leading edge during Drosophila dorsal closure." The Journal of cell biology 192(2): 335-348.

Laplante, C., S. M. Paul, G. J. Beitel and L. A. Nilson (2010). "Echinoid regulates tracheal morphology and fusion cell fate in Drosophila." Developmental dynamics : an official publication of the American Association of Anatomists 239(9): 2509-2519.

Laprise, P. and U. Tepass (2011). "Novel insights into epithelial polarity proteins in Drosophila." Trends Cell Biol 21(7): 401-408.

Leatherman, J. L. and T. A. Jongens (2003). "Transcriptional silencing and translational control: key features of early germline development." Bioessays 25(4): 326-335.

Lecuit, T. (2005). "Cell adhesion: sorting out cell mixing with echinoid?" Current biology: CB 15(13): R505-507.

Lecuit, T. and P. F. Lenne (2007). "Cell surface mechanics and the control of cell shape, tissue patterns and morphogenesis." Nat Rev Mol Cell Biol 8(8): 633-644.

Lecuit, T., R. Samanta and E. Wieschaus (2002). "slam encodes a developmental regulator of polarized membrane growth during cleavage of the Drosophila embryo." Developmental cell 2(4): 425-436.

Lecuyer, E. (2011). "High resolution fluorescent in situ hybridization in Drosophila." Methods in molecular biology 714: 31-47.

Lecuyer, E., H. Yoshida, N. Parthasarathy, C. Alm, T. Babak, T. Cerovina, T. R. Hughes, P. Tomancak and H. M. Krause (2007). "Global analysis of mRNA localization reveals a prominent role in organizing cellular architecture and function." Cell 131(1): 174-187.

Lehmann, R. (2016). "Germ Plasm Biogenesis--An Oskar-Centric Perspective." Curr Top Dev Biol 116: 679-707.

Leptin, M. (1995). "Drosophila gastrulation: from pattern formation to morphogenesis." Annu Rev Cell Dev Biol 11: 189-212.

Lerit, D. A. and E. R. Gavis (2011). "Transport of germ plasm on astral microtubules directs germ cell development in Drosophila." Current biology: CB 21(6): 439-448. 
Lin, S., S. Zhang and R. Cohen (2007). "Growl, a novel gurken RNA binding protein essential for dorsoventral patterning." Program and Abstracts. 48th Annual Drosophila Research Conference, Philadelphia, PA, 2007: 510C.

Little, S. C., K. S. Sinsimer, J. J. Lee, E. F. Wieschaus and E. R. Gavis (2015). "Independent and coordinate trafficking of single Drosophila germ plasm mRNAs." Nat Cell Biol.

Liu, H., J. Y. Wang, Y. Huang, Z. Li, W. Gong, R. Lehmann and R. M. Xu (2010). "Structural basis for methylarginine-dependent recognition of Aubergine by Tudor." Genes Dev 24(17): 1876-1881.

Macdonald, P. M., M. Kanke and A. Kenny (2016). "Community effects in regulation of translation." Elife $\mathbf{5}$.

Mahowald, A. P. (1962). "FINE STRUCTURE OF POLE CELLS AND POLAR GRANULES IN DROSOPHILA MELANOGASTER." Journal of Experimental Zoology 151(3): 201-\&.

Mahowald, A. P. (2001). "Assembly of the Drosophila germ plasm." Int Rev Cytol 203: 187-213.

Mani, R., R. P. St Onge, J. L. t. Hartman, G. Giaever and F. P. Roth (2008). "Defining genetic interaction." Proc Natl Acad Sci U S A 105(9): 3461-3466.

Marchler-Bauer, A., Y. Bo, L. Han, J. He, C. J. Lanczycki, S. Lu, F. Chitsaz, M. K. Derbyshire, R. C. Geer, N. R. Gonzales, M. Gwadz, D. I. Hurwitz, F. Lu, G. H. Marchler, J. S. Song, N. Thanki, Z. Wang, R. A. Yamashita, D. Zhang, C. Zheng, L. Y. Geer and S. H. Bryant (2017). "CDD/SPARCLE: functional classification of proteins via subfamily domain architectures." Nucleic Acids Res 45(D1): D200-D203.

Markussen, F. H., A. M. Michon, W. Breitwieser and A. Ephrussi (1995). "Translational control of oskar generates short OSK, the isoform that induces pole plasma assembly." Development 121(11): 3723-3732.

Martin, A. C., M. Kaschube and E. F. Wieschaus (2009). "Pulsed contractions of an actin-myosin network drive apical constriction." Nature 457(7228): 495-499.

Martin, K. C. and A. Ephrussi (2009). "mRNA localization: gene expression in the spatial dimension." Cell 136(4): 719-730.

Mason, F. M., M. Tworoger and A. C. Martin (2013). "Apical domain polarization localizes actin-myosin activity to drive ratchet-like apical constriction." Nat Cell Biol 15(8): 926-936. 
Mathews, W. R., D. Ong, A. B. Milutinovich and M. Van Doren (2006). "Zinc transport activity of Fear of Intimacy is essential for proper gonad morphogenesis and DEcadherin expression." Development 133(6): 1143-1153.

Mazumdar, A. and M. Mazumdar (2002). "How one becomes many: blastoderm cellularization in Drosophila melanogaster." Bioessays 24(11): 1012-1022.

McGill, M. A., R. F. McKinley and T. J. Harris (2009). "Independent cadherin-catenin and Bazooka clusters interact to assemble adherens junctions." J Cell Biol 185(5): 787796.

Micklem, D. R., J. Adams, S. Grunert and D. St Johnston (2000). "Distinct roles of two conserved Staufen domains in oskar mRNA localization and translation." EMBO J 19(6): 1366-1377.

Molitor, A. (2002). Isolierung und Charakterisierung des aeneas-Gens von Drosophila melanogaster. Dr. rer. nat. Dissertation, Technische Universität Carolo-Wilhelmina zu Braunschweig.

Morin, X., R. Daneman, M. Zavortink and W. Chia (2001). "A protein trap strategy to detect GFP-tagged proteins expressed from their endogenous loci in Drosophila." Proc Natl Acad Sci U S A 98(26): 15050-15055.

Nakamura, A., R. Amikura, M. Mukai, S. Kobayashi and P. F. Lasko (1996). "Requirement for a noncoding RNA in Drosophila polar granules for germ cell establishment." Science 274(5295): 2075-2079.

Nakamura, A., K. Sato and K. Hanyu-Nakamura (2004). "Drosophila cup is an eIF4E binding protein that associates with Bruno and regulates oskar mRNA translation in oogenesis." Dev Cell 6(1): 69-78.

Nakamura, A. and G. Seydoux (2008). "Less is more: specification of the germline by transcriptional repression." Development 135(23): 3817-3827.

Nezis, I. P., D. J. Stravopodis, I. Papassideri, M. Robert-Nicoud and L. H. Margaritis (2000). "Stage-specific apoptotic patterns during Drosophila oogenesis." Eur J Cell Biol 79(9): 610-620.

Ni, J. Q., R. Zhou, B. Czech, L. P. Liu, L. Holderbaum, D. Yang-Zhou, H. S. Shim, R. Tao, D. Handler, P. Karpowicz, R. Binari, M. Booker, J. Brennecke, L. A. Perkins, G. J. Hannon and N. Perrimon (2011). "A genome-scale shRNA resource for transgenic RNAi in Drosophila." Nat Methods 8(5): 405-407.

Nieuwburg, R., D. Nashchekin, M. Jakobs, A. P. Carter, P. Khuc Trong, R. E. Goldstein and D. St Johnston (2017). "Localised dynactin protects growing microtubules to deliver oskar mRNA to the posterior cortex of the Drosophila oocyte." Elife 6. 
Ollion, J., J. Cochennec, F. Loll, C. Escude and T. Boudier (2013). "TANGO: a generic tool for high-throughput 3D image analysis for studying nuclear organization." Bioinformatics 29(14): 1840-1841.

Padash Barmchi, M., S. Rogers and U. Hacker (2005). "DRhoGEF2 regulates actin organization and contractility in the Drosophila blastoderm embryo." J Cell Biol 168(4): 575-585.

Palancade, B. and O. Bensaude (2003). "Investigating RNA polymerase II carboxylterminal domain (CTD) phosphorylation." Eur J Biochem 270(19): 3859-3870.

Patel, N. H. (1994). "Imaging neuronal subsets and other cell types in whole-mount Drosophila embryos and larvae using antibody probes." Methods Cell Biol 44: 445-487.

Rabinowitz, M. (1941). "Studies on the cytology and early embryology of the egg of Drosophila melanogaster." Journal of Morphology 69(1): 1-49.

Rangan, P., M. DeGennaro, K. Jaime-Bustamante, R. X. Coux, R. G. Martinho and R. Lehmann (2009). "Temporal and spatial control of germ-plasm RNAs." Current biology: CB 19(1): 72-77.

Rauzi, M., U. Krzic, T. E. Saunders, M. Krajnc, P. Ziherl, L. Hufnagel and M. Leptin (2015). "Embryo-scale tissue mechanics during Drosophila gastrulation movements." Nat Commun 6: 8677.

Rauzi, M., P. F. Lenne and T. Lecuit (2010). "Planar polarized actomyosin contractile flows control epithelial junction remodelling." Nature 468(7327): 1110-1114.

Rawlins, E. L., B. Lovegrove and A. P. Jarman (2003a). "Echinoid facilitates Notch pathway signalling during Drosophila neurogenesis through functional interaction with Delta." Development 130(26): 6475-6484.

Rawlins, E. L., N. M. White and A. P. Jarman (2003b). "Echinoid limits R8 photoreceptor specification by inhibiting inappropriate EGF receptor signalling within R8 equivalence groups." Development 130(16): 3715-3724.

Renault, A. D., P. S. Kunwar and R. Lehmann (2010). "Lipid phosphate phosphatase activity regulates dispersal and bilateral sorting of embryonic germ cells in Drosophila." Development 137(11): 1815-1823.

Renault, A. D., Y. J. Sigal, A. J. Morris and R. Lehmann (2004). "Soma-germ line competition for lipid phosphate uptake regulates germ cell migration and survival." Science 305(5692): 1963-1966.

Reuter, R., B. Grunewald and M. Leptin (1993). "A role for the mesoderm in endodermal migration and morphogenesis in Drosophila." Development 119(4): 11351145 . 
Rorth, P. (1998). "Gal4 in the Drosophila female germline." Mech Dev 78(1-2): 113118.

Roth, S. and J. A. Lynch (2009). "Symmetry breaking during Drosophila oogenesis." Cold Spring Harb Perspect Biol 1(2): a001891.

Roth, S., F. S. Neuman-Silberberg, G. Barcelo and T. Schupbach (1995). "cornichon and the EGF receptor signaling process are necessary for both anterior-posterior and dorsal-ventral pattern formation in Drosophila." Cell 81(6): 967-978.

Samarage, C. R., M. D. White, Y. D. Alvarez, J. C. Fierro-Gonzalez, Y. Henon, E. C. Jesudason, S. Bissiere, A. Fouras and N. Plachta (2015). "Cortical Tension Allocates the First Inner Cells of the Mammalian Embryo." Dev Cell 34(4): 435-447.

Santos, A. C. and R. Lehmann (2004a). "Germ cell specification and migration in Drosophila and beyond." Current biology: CB 14(14): R578-589.

Santos, A. C. and R. Lehmann (2004b). "Isoprenoids control germ cell migration downstream of HMGCoA reductase." Developmental cell 6(2): 283-293.

Seifert, J. R. and R. Lehmann (2012). "Drosophila primordial germ cell migration requires epithelial remodeling of the endoderm." Development 139(12): 2101-2106.

Serbus, L. R., B. J. Cha, W. E. Theurkauf and W. M. Saxton (2005). "Dynein and the actin cytoskeleton control kinesin-driven cytoplasmic streaming in Drosophila oocytes." Development 132(16): 3743-3752.

Seydoux, G. and M. A. Dunn (1997). "Transcriptionally repressed germ cells lack a subpopulation of phosphorylated RNA polymerase II in early embryos of Caenorhabditis elegans and Drosophila melanogaster." Development 124(11): 21912201.

Shulman, J. M., R. Benton and D. St Johnston (2000). "The Drosophila homolog of C. elegans PAR-1 organizes the oocyte cytoskeleton and directs oskar mRNA localization to the posterior pole." Cell 101(4): 377-388.

Sigal, Y. J., M. I. McDermott and A. J. Morris (2005). "Integral membrane lipid phosphatases/phosphotransferases: common structure and diverse functions." Biochem J 387(Pt 2): 281-293.

Simon, B., P. Masiewicz, A. Ephrussi and T. Carlomagno (2015). "The structure of the SOLE element of oskar mRNA." RNA 21(8): 1444-1453.

Sinsimer, K. S., R. A. Jain, S. Chatterjee and E. R. Gavis (2011). "A late phase of germ plasm accumulation during Drosophila oogenesis requires lost and rumpelstiltskin." Development 138(16): 3431-3440. 
Sinsimer, K. S., J. J. Lee, S. Y. Thiberge and E. R. Gavis (2013). "Germ plasm anchoring is a dynamic state that requires persistent trafficking." Cell Rep 5(5): 11691177.

Slaidina, M. and R. Lehmann (2017). "Quantitative Differences in a Single Maternal Factor Determine Survival Probabilities among Drosophila Germ Cells." Curr Biol 27(2): 291-297.

Snee, M. J., D. Harrison, N. Yan and P. M. Macdonald (2007). "A late phase of Oskar accumulation is crucial for posterior patterning of the Drosophila embryo, and is blocked by ectopic expression of Bruno." Differentiation 75(3): 246-255.

Snee, M. J. and P. M. Macdonald (2009). "Dynamic organization and plasticity of sponge bodies." Developmental dynamics : an official publication of the American Association of Anatomists 238(4): 918-930.

Sonnenblick, B. P. (1941). "Germ Cell Movements and Sex Differentiation of the Gonads in the Drosophila Embryo." Proceedings of the National Academy of Sciences of the United States of America 27(10): 484-489.

Spradling, A. C., M. de Cuevas, D. Drummond-Barbosa, L. Keyes, M. Lilly, M. Pepling and T. Xie (1997). "The Drosophila germarium: stem cells, germ line cysts, and oocytes." Cold Spring Harb Symp Quant Biol 62: 25-34.

St Johnston, D., D. Beuchle and C. Nusslein-Volhard (1991). "Staufen, a gene required to localize maternal RNAs in the Drosophila egg." Cell 66(1): 51-63.

St Johnston, D., W. Driever, T. Berleth, S. Richstein and C. Nusslein-Volhard (1989). "Multiple steps in the localization of bicoid RNA to the anterior pole of the Drosophila oocyte." Development 107 Suppl: 13-19.

Staller, M. V., D. Yan, S. Randklev, M. D. Bragdon, Z. B. Wunderlich, R. Tao, L. A. Perkins, A. H. Depace and N. Perrimon (2013). "Depleting gene activities in early Drosophila embryos with the "maternal-Gal4-shRNA" system." Genetics 193(1): 51-61.

Starz-Gaiano, M., N. K. Cho, A. Forbes and R. Lehmann (2001). "Spatially restricted activity of a Drosophila lipid phosphatase guides migrating germ cells." Development 128(6): 983-991.

Starz-Gaiano, M. and R. Lehmann (2001). "Moving towards the next generation." Mechanisms of development 105(1-2): 5-18.

Stoiber, M. H., S. Olson, G. E. May, M. O. Duff, J. Manent, R. Obar, K. G. Guruharsha, P. J. Bickel, S. Artavanis-Tsakonas, J. B. Brown, B. R. Graveley and S. E. Celniker (2015). "Extensive cross-regulation of post-transcriptional regulatory networks in Drosophila." Genome Res 25(11): 1692-1702. 
Strome, S. and D. Updike (2015). "Specifying and protecting germ cell fate." Nat Rev Mol Cell Biol 16(7): 406-416.

Tanaka, T., Y. Kato, K. Matsuda, K. Hanyu-Nakamura and A. Nakamura (2011). "Drosophila Mon2 couples Oskar-induced endocytosis with actin remodeling for cortical anchorage of the germ plasm." Development 138(12): 2523-2532.

Tautz, D. and C. Pfeifle (1989). "A non-radioactive in situ hybridization method for the localization of specific RNAs in Drosophila embryos reveals translational control of the segmentation gene hunchback." Chromosoma 98(2): 81-85.

Tepass, U. (1996). "Crumbs, a component of the apical membrane, is required for zonula adherens formation in primary epithelia of Drosophila." Dev Biol 177(1): 217225 .

Tepass, U. (2012). "The apical polarity protein network in Drosophila epithelial cells: regulation of polarity, junctions, morphogenesis, cell growth, and survival." Annu Rev Cell Dev Biol 28: 655-685.

Tepass, U., G. Tanentzapf, R. Ward and R. Fehon (2001). "Epithelial cell polarity and cell junctions in Drosophila." Annu Rev Genet 35: 747-784.

Thomson, T., N. Liu, A. Arkov, R. Lehmann and P. Lasko (2008). "Isolation of new polar granule components in Drosophila reveals P body and ER associated proteins." Mechanisms of development 125(9-10): 865-873.

Trcek, T., M. Grosch, A. York, H. Shroff, T. Lionnet and R. Lehmann (2015). "Drosophila germ granules are structured and contain homotypic mRNA clusters." Nat Commun 6: 7962.

Umetsu, D., B. Aigouy, M. Aliee, L. Sui, S. Eaton, F. Julicher and C. Dahmann (2014). "Local increases in mechanical tension shape compartment boundaries by biasing cell intercalations." Curr Biol 24(15): 1798-1805.

Van Doren, M., H. T. Broihier, L. A. Moore and R. Lehmann (1998a). "HMG-CoA reductase guides migrating primordial germ cells." Nature 396(6710): 466-469.

Van Doren, M., W. R. Mathews, M. Samuels, L. A. Moore, H. T. Broihier and R. Lehmann (2003). "fear of intimacy encodes a novel transmembrane protein required for gonad morphogenesis in Drosophila." Development 130(11): 2355-2364.

Van Doren, M., A. L. Williamson and R. Lehmann (1998b). "Regulation of zygotic gene expression in Drosophila primordial germ cells." Curr Biol 8(4): 243-246.

Vanzo, N., A. Oprins, D. Xanthakis, A. Ephrussi and C. Rabouille (2007). "Stimulation of endocytosis and actin dynamics by Oskar polarizes the Drosophila oocyte." Dev Cell 12(4): 543-555. 
Vazquez-Pianzola, P., H. Urlaub and B. Suter (2011). "Pabp binds to the osk 3'UTR and specifically contributes to osk mRNA stability and oocyte accumulation." Dev Biol 357(2): 404-418.

Vogel, C., S. A. Teichmann and C. Chothia (2003). "The immunoglobulin superfamily in Drosophila melanogaster and Caenorhabditis elegans and the evolution of complexity." Development 130(25): 6317-6328.

Warn, R. M. and A. Warn (1986). "Microtubule arrays present during the syncytial and cellular blastoderm stages of the early Drosophila embryo." Exp Cell Res 163(1): 201210.

Wei, S. Y., L. M. Escudero, F. Yu, L. H. Chang, L. Y. Chen, Y. H. Ho, C. M. Lin, C. S. Chou, W. Chia, J. Modolell and J. C. Hsu (2005). "Echinoid is a component of adherens junctions that cooperates with DE-Cadherin to mediate cell adhesion." Dev Cell 8(4): 493-504.

Wenzl, C., S. Yan, P. Laupsien and J. Grosshans (2010). "Localization of RhoGEF2 during Drosophila cellularization is developmentally controlled by Slam." Mechanisms of development 127(7-8): 371-384.

Wheatley, S., S. Kulkarni and R. Karess (1995). "Drosophila nonmuscle myosin II is required for rapid cytoplasmic transport during oogenesis and for axial nuclear migration in early embryos." Development 121(6): 1937-1946.

Wodarz, A., A. Ramrath, U. Kuchinke and E. Knust (1999). "Bazooka provides an apical cue for Inscuteable localization in Drosophila neuroblasts." Nature 402(6761): 544-547.

Worringer, K. A., F. Chu and B. Panning (2009). "The zinc finger protein Zn72D and DEAD box helicase Belle interact and control maleless mRNA and protein levels." BMC molecular biology 10: 33 .

Zalokar, M. (1976). "Autoradiographic study of protein and RNA formation during early development of Drosophila eggs." Dev Biol 49(2): 425-437.

Zimyanin, V. L., K. Belaya, J. Pecreaux, M. J. Gilchrist, A. Clark, I. Davis and D. St Johnston (2008). "In vivo imaging of oskar mRNA transport reveals the mechanism of posterior localization." Cell 134(5): 843-853. 



\section{Curriculum vitae}

The Curriculum vitae is not available in the electronic version. 



\section{Appendix l: Protocols}

\section{Antibody staining with embryos}

- Wash embryos in $600 \mu \mathrm{l}$ methanol and $300 \mu \mathrm{l}$ PBST for $10 \mathrm{~min}$

- Wash embryos in $300 \mu \mathrm{l}$ methanol and $600 \mu \mathrm{l}$ PBST for $10 \mathrm{~min}$

- Rinse embryos 3 times with $1 \mathrm{ml}$ PBST

- wash embryos 3 times for 15 min with PBST

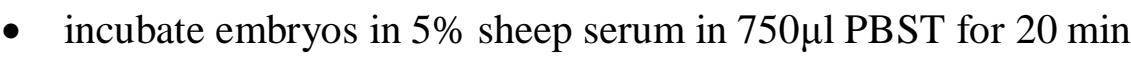

- repeat blocking step once

- add primary antibody/antibodies in $2 \%$ sheep serum in $750 \mu 1$ PBST

- incubate over night at $4^{\circ} \mathrm{C}$ rotating

- rinse $3 \mathrm{x}$ with $1 \mathrm{ml}$ PBST

- wash 10min, 20min, 30min with $1 \mathrm{ml}$ PBST

- incubate embryos in $2 \%$ sheep serum in $750 \mu \mathrm{l}$ PBST for $20 \mathrm{~min}$

- add fluorescent secondary antibody/antibodies (1:500), in 750 $\mu$ PBST with 2\% sheep serum

- if needed, add DAPI now (from 500X stock, use $3 \mu \mathrm{l}$ in $750 \mu \mathrm{l}$ volume)

- incubate $2 \mathrm{~h}$ at $\mathrm{RT}$ rotating

- rinse 3-5 times with $1 \mathrm{ml}$ PBST

- wash 10min, 20min, 30min with $1 \mathrm{ml}$ PBST.

\section{Embryo fixation}

- remove dead flies from apple juice agar plate

- optional: remove yeast paste from AJ agar plate

- cover plate with water

- use brush to gently detach embryos from agar

- transfer water-embryo suspension into collection sieve

- rinse with water to remove yeast

- incubate sieve with embryos in $50 \%$ bleach solution for $3 \mathrm{~min}$

- rinse thoroughly with water

- prepare glass vials with $1.5 \mathrm{ml}$ fix solution and $6 \mathrm{ml}$ heptane

- remove water from embryo collection sieve

- transfer embryos to $20 \mathrm{ml}$ scintillation vial

- incubate for $20 \mathrm{~min}$ on shaker

- gently remove lower phase with pasteur pipette without removing embryos 
- add $6 \mathrm{ml}$ methanol

- shake heavily for $10-20 \mathrm{sec}$

- let embryos sink down

- remove upper phase and embryos in interphase completely

- rinse 3x with methanol

- transfer embryos to $1.5 \mathrm{ml}$ test tube

- store at $-20^{\circ} \mathrm{C}$

\section{Create antisense RNA probes}

- use $5 \mu \mathrm{g}$ plasmid DNA

- linearize for $2 \mathrm{~h}$ to $3 \mathrm{~h}$ at $37^{\circ} \mathrm{C}$

- purify using QIAquick Nucleotide Removal Kit (Qiagen) columns

- elute in $30 \mu 1$ to $40 \mu 1 \mathrm{H}_{2} 0$

- measure DNA concentration with NanoDrop

- use DIG RNA Labeling Kit (Roche)

- $\quad$ set up reaction (10 $\mu 1$ end volume):

- $1 \mu \mathrm{g}$ linearized plasmid DNA (ca. $6 \mu \mathrm{l})$

- $1 \mu 1$ transcription buffer $10 \mathrm{x}$

- $1 \mu 1$ NTP Labeling Mixture

- $1 \mu 1$ RNase inhibitor

- $1 \mu 1$ RNA polymerase (T7, T3 or SP6)

- incubate $2 \mathrm{~h}$ at $37^{\circ} \mathrm{C}$

- load $1 \mu \mathrm{l}$ of reaction mixture $\left(+9 \mu 1 \mathrm{H}_{2} \mathrm{O},+2 \mu 1\right.$ loading dye) onto $1 \%$ agarose gel to check reaction

- add RNAse free water to sample up to $50 \mu 1$

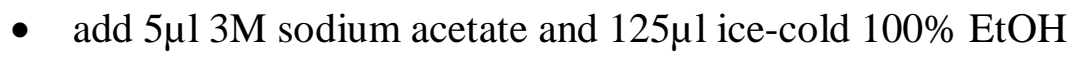

- place at $-70^{\circ} \mathrm{C}$ overnight

- centrifuge at max speed at cooled tabletop centrifuge for $20 \mathrm{~min}$

- remove supernatant, wash with $150 \mu 170 \% \mathrm{EtOH}$

- centrifuge at max speed at cooled tabletop centrifuge for $20 \mathrm{~min}$

- remove EtOH, dry pellets in speed-vac (2-5 min)

- resuspend probe in $100 \mu 1$ Hybe buffer

- store at $-20^{\circ} \mathrm{C}$ 


\section{FISH}

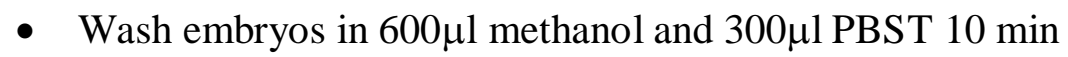

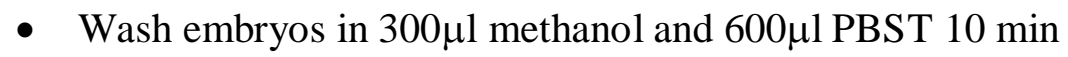

- Rinse embryos 3 times with $1 \mathrm{ml}$ PBST

- Wash embryos 3 times 15 min with PBST

- Wash 1 time in HybB/PBST 1:1 for 20 min at RT

- Wash 3 times 20 min in $\mathrm{HybB}$ at $57^{\circ} \mathrm{C}$ (water bath)

- remove HybB; leave $\sim 40 \mu 1$ of buffer on embryos

- add $1 \mu 1$ of labeled (DIG) probe, mix gently

- incubate at $57^{\circ} \mathrm{C}$ over night (water bath)

- discard probe-HybB mix

- rinse $3 \mathrm{x}$ with $1 \mathrm{ml} \mathrm{HybB}\left(57^{\circ} \mathrm{C}\right)$

- wash $10 \mathrm{~min}, 20 \mathrm{~min}, 30 \mathrm{~min}$ with $1 \mathrm{ml} \mathrm{HybB}, 57^{\circ} \mathrm{C}$ (water bath)

- wash 20min with $1 \mathrm{ml} \mathrm{HybB/PBST} \mathrm{1:1} \mathrm{at} \mathrm{RT}$

- rinse $3 \mathrm{x}$ with $1 \mathrm{ml}$ PBST

- wash 10min, 20min, 30min with $1 \mathrm{ml}$ PBST

- add primary antibody (sheep anti-DIG, HRP coupled) 1:200 to 1:500

- incubate $1.5 \mathrm{~h}$ at RT

- Rinse 3 times with PBST

- wash $10 \mathrm{~min}, 20 \mathrm{~min}, 30 \mathrm{~min}$ with $1 \mathrm{ml}$ PBST

- take as much PBST from embryos as possible

- rinse 2 times with $100 \mu$ l Amplification diluent (TSA kit)

- Add $2 \mu 1$ of the Fluorophor solution to the dilution solution

- Stir embryos up a bit and incubate in the dark for 10 to $20 \mathrm{~min}$

- Rinse at least 3-10 times with PBST

- wash 10min, 20min, 30min with $1 \mathrm{ml}$ PBST

- proceed with antibody staining or desired mounting protocol 



\section{Appendix II: Fiii macros}

\section{Macro for measuring pCTD intensity of SNUPs}

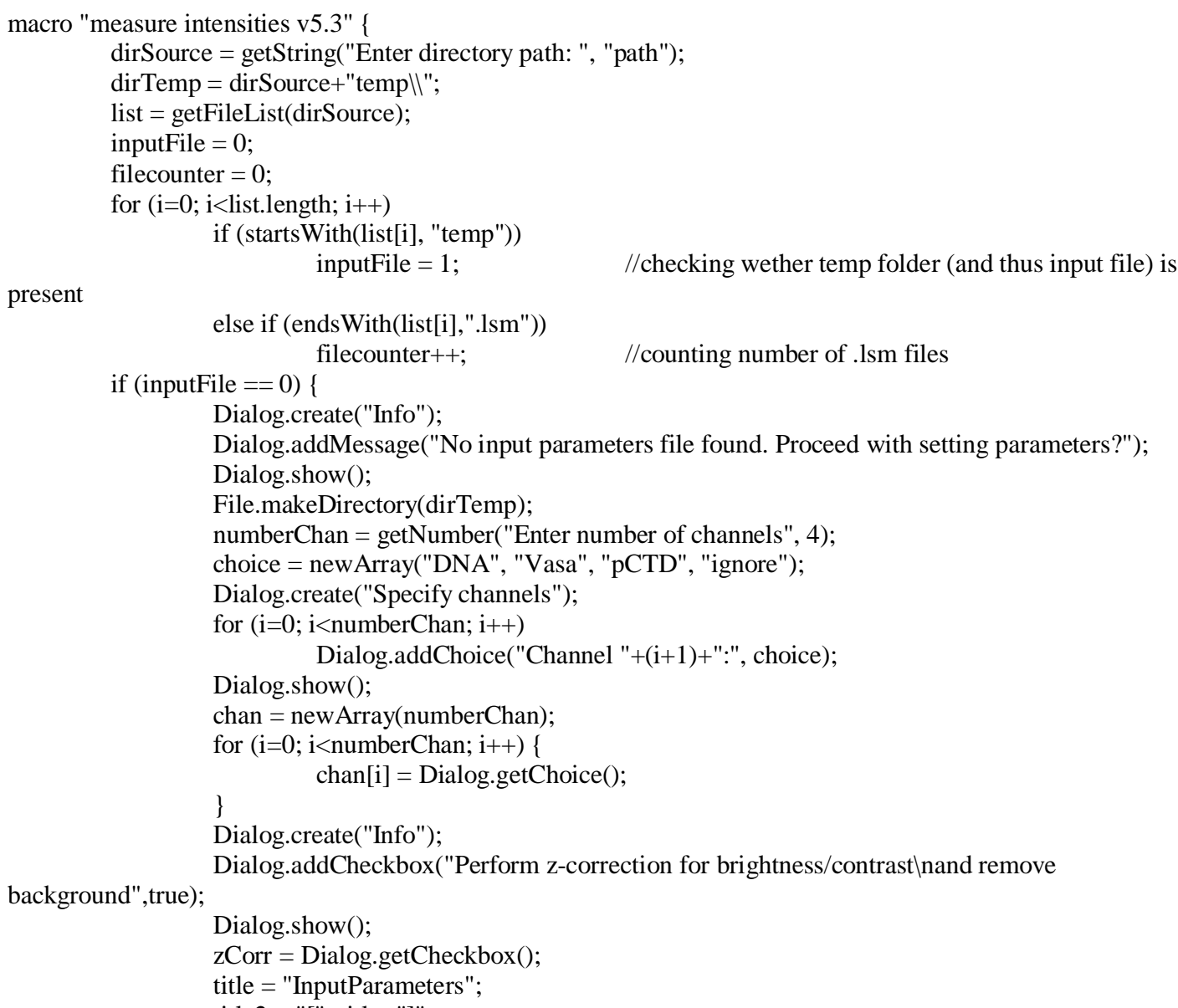

//now opens each file one after another, splitting channels, asks for thresholds, stores the values in an table and the channel files in Itemp folder

run("New... ", "name="+title+" type=Table width=950 height=600");

print(title2,

"|\Headings:filenameltthresholdDNA|tthresholdVasaltthresholdpCTD $\mid$ tlowerBorderltcomment|tblank2");

for $(\mathrm{i}=0$; $\mathrm{i}<$ list.length; $\mathrm{i}++)\{$

if (endsWith(list[i],".lsm")) \{

open(dirSource+list[i]);

run("Split Channels");

for $(\mathrm{k}=0 ; \mathrm{k}<$ numberChan; $\mathrm{k}++)\{\quad$ //perform actions on

channels

if (chan[k] == "ignore") \{ //close all channels labeled as "ignore" selectWindow("C"+(k+1)+"-"+list[i]); close ();

\} else if $(($ chan $[\mathrm{k}]==$ "DNA") \&\& (zCorr $==$ true $))\{$ //perform zcorrection \& background removal in DNA and VASA channels, if checkbox was checked selectWindow("C"+(k+1)+"-"+list[i]); runMacro("F:IIGWDG-

cloud\\Fiji.app \|macros \lown $\mid$ z_correction_brightness.ijm"); 
//gaussian smooth

substantially!!
run("Apply LUT", "stack");

run("Gaussian Blur...", "sigma=2 stack");

setMinAndMax $(5,255) ; / /$ this reduces background

run("Apply LUT", "stack");

\} else if $((\operatorname{chan}[\mathrm{k}]==$ "Vasa") \&\& $(\mathrm{zCorr}==$ true $))\{$

selectWindow("C"+(k+1)+"-"+list[i]); runMacro("F:IIGWDG-

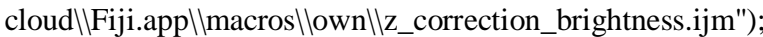

setMinAndMax $(15,255) ; \quad / /$ remove background.threshold 20

run("Apply LUT", "stack");

run("Gaussian Blur...", "sigma=2 stack");

//gaussian smooth

substantially!!

setMinAndMax(10. 255); //this reduces background

run("Apply LUT", "stack");

\}

waitForUser("Set thresholds", "Adjust the threshold for each channel

individually. Set the lower y-border in the pCTD channel. Then click ok. InIf no threshold is set in Vasa channel this stack will be skipped during measurement procedure.");

Dialog.create("Set parameters");

Dialog.addMessage("Optional: Leave a comment or mark the stack for being

skipped during measurement.");

Dialog.setInsets(15, 50. 5);

Dialog.addString("comments", "");

Dialog.setInsets(15, 50. 5);

Dialog.addCheckbox("skip this stack",false);

Dialog.show();

comment $=$ Dialog.getString () ;

skip $=$ Dialog.getCheckbox () ;

for $(j=0 ; j<$ numberChan; $j++)\{$

$$
\text { if ( } \operatorname{chan}[\mathrm{j}]==\text { "DNA") \{ }
$$

selectWindow("C"+(j+1)+"-"+list[i]);

getThreshold(1,thresholdDNA);

resetThreshold();

if ( kip $==$ false)

saveAs("tiff",dirTemp+"DNA_"+list[i]);

//save channel file only if stack is not marked as "skip"

close();

$$
\text { \} }
$$

$$
\text { else if }(\text { chan }[\mathrm{j}]==\text { "Vasa") }\{
$$$$
\text { selectWindow("C"+(j+1)+"-"+list[i]); }
$$

getThreshold(1,thresholdVasa);

resetThreshold();

if ( skip $==$ false) close ();

saveAs("tiff",dirTemp+"Vasa_"+list[i]);

\}

else if $(\operatorname{chan}[\mathrm{j}]==$ "pCTD") \{

selectWindow("C"+(j+1)+"-"+list[i]);

getThreshold(1,thresholdpCTD);

resetThreshold();

getLine $(x 1, y 1, x 2, y 2$, lineWidth);

if $(x 1 !=-1)$

lowerBorder $=\operatorname{round}((\mathrm{y} 1+\mathrm{y} 2) / 2)$;

else

lowerBorder = "NaN"; 


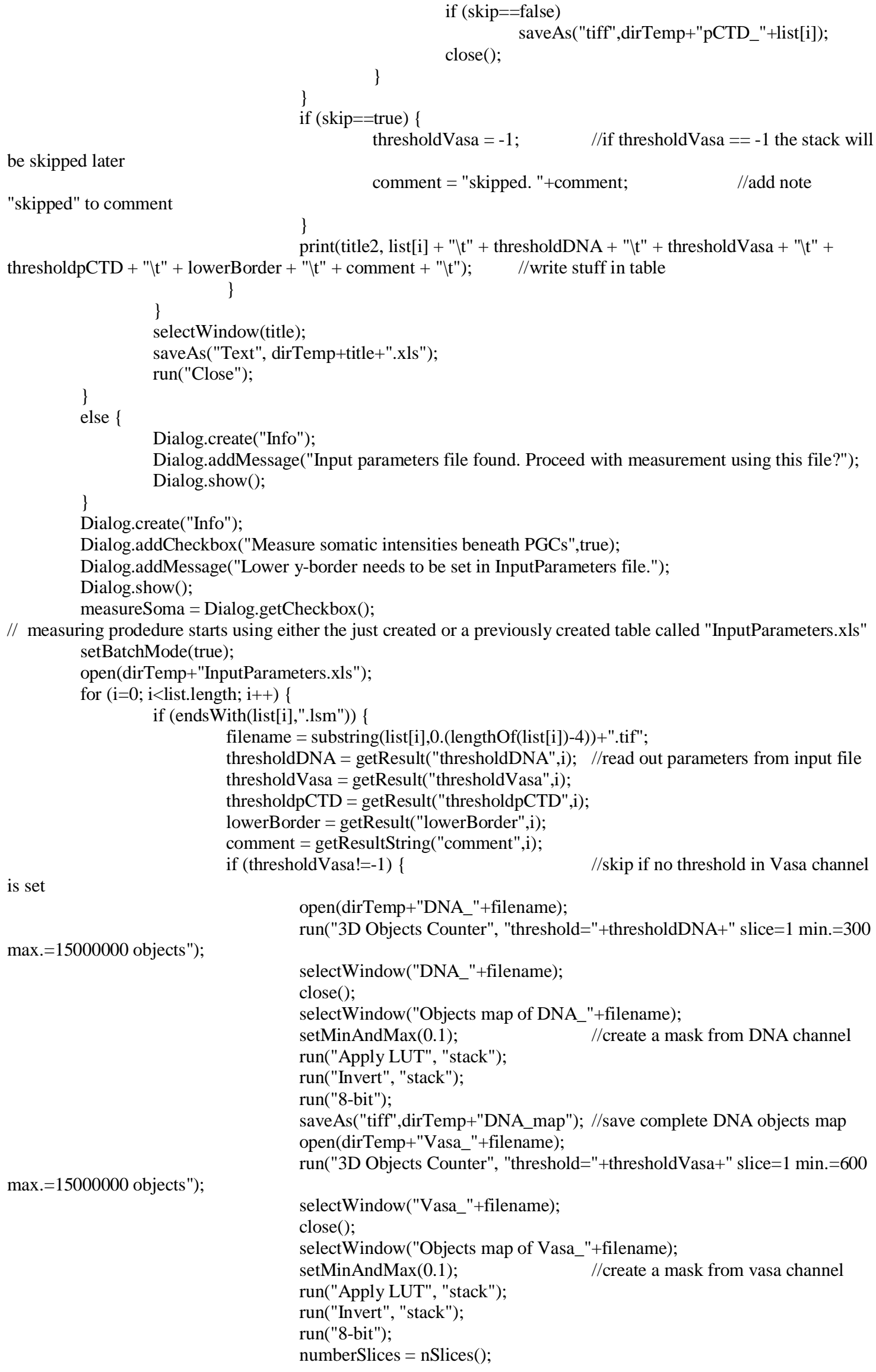

//skip if no threshold in Vasa channel

is set

max.=15000000 objects");

$\max .=15000000$ objects");

open(dirTemp+"DNA_"+filename);

run("3D Objects Counter", "threshold="+thresholdDNA+" slice=1 min.=300

selectWindow("DNA_"+filename);

close ();

selectWindow("Objects map of DNA_"+filename);

setMinAndMax $(0.1)$; //create a mask from DNA channel

run("Apply LUT", "stack");

run("Invert", "stack");

run("8-bit");

saveAs("tiff",dirTemp+"DNA_map"); //save complete DNA objects map open(dirTemp+"Vasa_"+filename);

run("3D Objects Counter", "threshold="+thresholdVasa+" slice=1 min.=600

selectWindow("Vasa_"+filename);

close ();

selectWindow("Objects map of Vasa_"+filename);

setMinAndMax $(0.1) ; \quad$ //create a mask from vasa channel

run("Apply LUT", "stack");

run("Invert", "stack");

run("8-bit");

numberSlices $=$ nSlices () ; 
domain

pCTD channel and get histogram for each slice

histogram counts and save as array[256]

to DNA channel

domain

\}

close();

Vasa channel and invert
DNA channel

signals INSIDE vasa domain

for $(j=0 ; j<$ numberSlices; $j++)\{$

selectWindow("Objects map of Vasa_"+filename);

setSlice $(j+1)$;

run("Create Selection"); $\quad$ //apply that mask (slice by slice) to

selection $=$ selectionType () ;

if (selection $!=-1)\{$

selectWindow("DNA_map.tif");

setSlice $(\mathrm{j}+1)$;

run("Restore Selection");

run("Make Inverse");

run("Fill", "slice"); // black eveything OUTSIDE vasa

selectWindow("DNA_map.tif");

run("Select None");

setMinAndMax $(0.1) ; \quad$ //create mask from DNA_map channel for

run("Apply LUT", "stack");

HistoIn = newArray $(256)$;

open(dirTemp+"pCTD_"+filename);

for $(j=0 ; j<$ numberSlices; $j++)\{$

selectWindow("DNA_map.tif");

setSlice $(j+1)$;

run("Create Selection");

selection $=$ selectionType () ;

if (selection != -1) \{

selectWindow("pCTD_"+filename); //apply mask to

setSlice $(\mathrm{j}+1)$;

run("Restore Selection");

getHistogram(values, counts, 256);

for $(\mathrm{k}=0 ; \mathrm{k}<256 ; \mathrm{k}++)$

HistoIn[k] = HistoIn[k] + counts[k]; //sum up

\}

selectWindow("DNA map.tif"); $\quad$ //close modified DNA map

selectWindow("Objects map of Vasa_"+filename); //select mask from

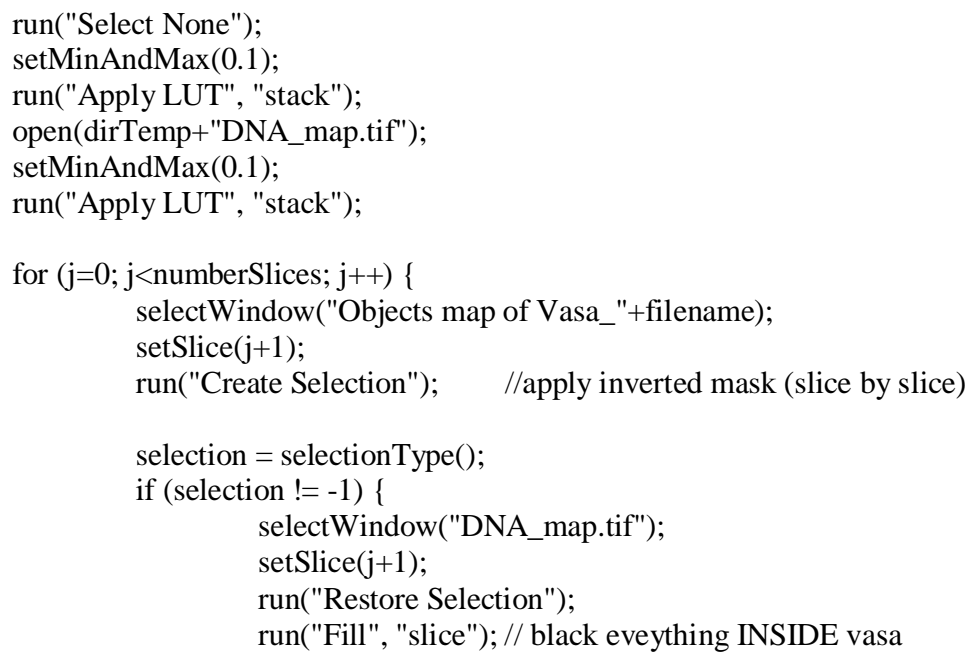


selectWindow("DNA_map.tif");

run("Select None");

setMinAndMax (0.1);

//create mask from

DNA channel for signals OUTSIDE vasa domain

$$
\text { run("Apply LUT", "stack"); }
$$

saveAs("tiff",dirTemp+"DNA_map"); ～//save modified DNA_map.

now DNA_map only contains somatic nuclei!!

HistoOut = newArray $(256)$;

for $(j=0 ; j<$ numberSlices; $j++)\{$

selectWindow("DNA_map.tif");

setSlice $(j+1)$;

run("Create Selection");

selection $=$ selectionType () ;

if (selection $!=-1$ )

selectWindow("pCTD_"+filename); //apply mask to

pCTD channel and get histogram for each slice

setSlice $(j+1)$;

run("Restore Selection");

getHistogram(values, counts, 256);

for $(\mathrm{k}=0 ; \mathrm{k}<256 ; \mathrm{k}++)$

HistoOut $[\mathrm{k}]=$ HistoOut $[\mathrm{k}]+$ counts $[\mathrm{k}]$;

//sum up histogram counts and save as array[256]

\}

\}

selectWindow("DNA_map.tif"); //close modified DNA_map

close ();

// $\quad$ measuring SNUP activity starts

if (measureSoma $==$ true) \{

if (lowerBorder $==-1$ )

exit("Lower y-border not set. Check InputParameters

file.");

selectWindow("Objects map of Vasa_"+filename);

run("Select None");

setMinAndMax $(0.1)$;

run("Apply LUT", "stack");

for $(j=0 ; j<$ numberSlices; $j++)\{$

//

selectWindow("Objects map of Vasa_"+filename);

setSlice $(j+1)$;

run("Create Selection");

selection = selectionType () ;

if (selection !=-1) \{

getSelectionBounds(x, y, width, height);

//get coordinates of bounding box of selection

$$
\text { if (width <3) }
$$

makeRectangle $(\mathrm{x}, 0$. width,

lowerBorder); //make new selection with lower y-border and $\mathrm{x}$-values from vasa mask else

makeRectangle((x+(width*15/100)),0.(width-(width*15/100)),lowerBorder); //measure only in under-vasaarea minus $15 \%$ from left and right

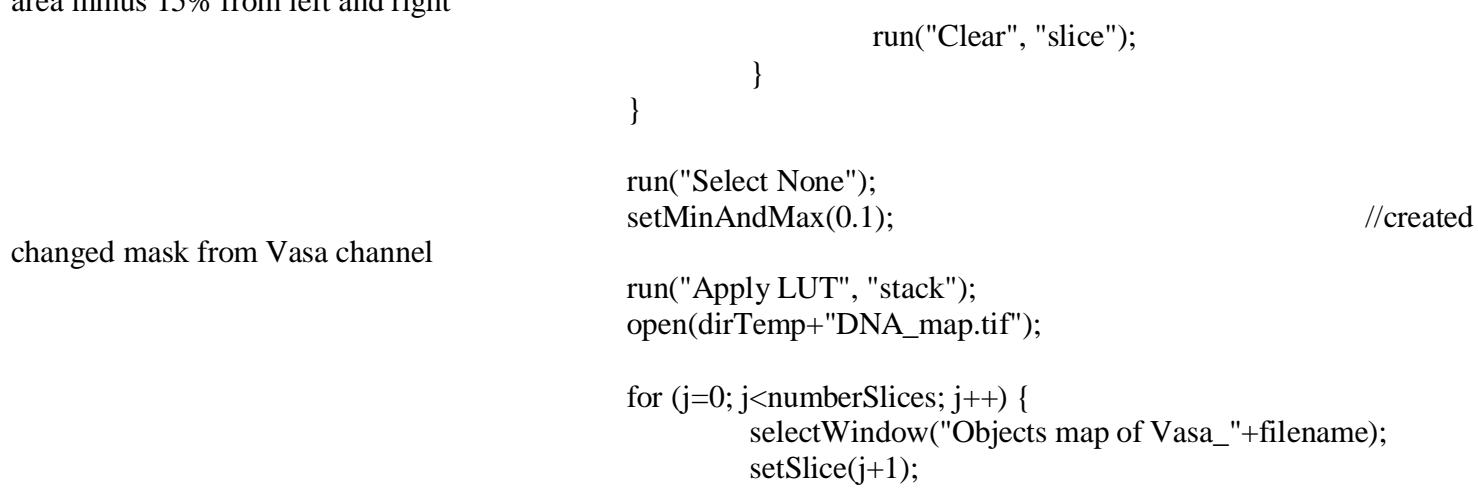


by slice) to DNA channel (vasa domain already blacked)

run("Create Selection"); / / apply changed mask (slice

selection $=$ selectionType () ;

if (selection != -1)

selectWindow("DNA_map.tif");

setSlice $(\mathrm{j}+1)$;

run("Restore Selection");

run("Fill", "slice"); // black eveything INSIDE

under-PGC area

signals OUTSIDE under-PGC area

mask to pCTD channel and get histogram for each slice

selectWindow("DNA_map.tif");

run("Select None");

setMinAndMax (0.1); //create mask from DNA channel for

run("Apply LUT", "stack");

SomaNotUnder = newArray (256);

for $(\mathrm{j}=0 ; \mathrm{j}<$ numberSlices; $\mathrm{j}++)$

selectWindow("DNA_map.tif");

setSlice $(j+1)$;

run("Create Selection");

selection $=$ selectionType () ;

if (selection != -1)

selectWindow("pCTD_"+filename); //apply

setSlice $(j+1)$;

run("Restore Selection");

getHistogram(values, counts, 256);

for $(\mathrm{k}=0 ; \mathrm{k}<256 ; \mathrm{k}++)$

SomaNotUnder $[\mathrm{k}]=$ SomaNotUnder $[\mathrm{k}]$

+ counts[k]; $\quad$ //sum up histogram counts and save as array[256]

DNA_map

selectWindow("DNA_map.tif");

//close changed

close();

selectWindow("Objects map of Vasa_"+filename); / / /select

changed mask from Vasa channel and invert

run("Select None");

run("Invert", "stack");

setMinAndMax $(0.1)$;

run("Apply LUT", "stack");

open(dirTemp+"DNA_map.tif");

//open

DNA_map, with Vasa domain already blacked

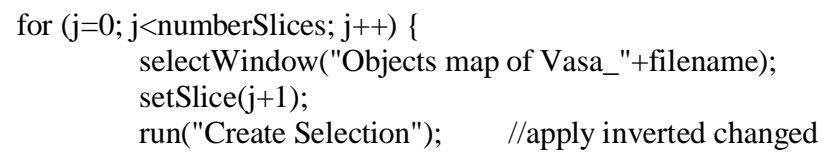

mask (slice by slice) to DNA channel (vasa domain already blacked) selection $=$ selectionType () ;

if (selection != -1)

selectWindow("DNA_map.tif");

setSlice $(j+1)$;

run("Restore Selection");

run("Fill", "slice"); // black eveything OUTSIDE

under-PGC area

\}

selectWindow("DNA_map.tif");

run("Select None");

setMinAndMax $(0.1) ; \quad$ //create mask from DNA channel for

signals INSIDE under-PGC area

run("Apply LUT", "stack");

SomaUnder $=$ new Array $(256)$; 
for $(j=0 ; j<$ numberSlices; $j++)\{$

selectWindow("DNA_map.tif");

setSlice $(j+1)$;

run("Create Selection");

selection $=$ selectionType () ;

if (selection != -1) \{

mask to pCTD channel and get histogram for each slice

selectWindow("pCTD_"+filename); //apply

setSlice $(j+1)$;

run("Restore Selection");

getHistogram(values, counts, 256);

for $(\mathrm{k}=0 ; \mathrm{k}<256 ; \mathrm{k}++)$

counts[k];

//sum up histogram counts and save as array[256]

SomaUnder $[\mathrm{k}]=$ SomaUnder $[\mathrm{k}]+$

\}

\}

close();

close();

close ();

NumberVoxelsIn $=0$;

TotalValueIn $=0$;

NumberVoxelsOut $=0$;

TotalValueOut $=0$;

NumberSomaNotUnder $=0$;

TotalSomaNotUnder $=0$;

NumberSomaUnder $=0$;

TotalSomaUnder $=0$;

pCTD threshold defines the background lvl of pCTD channel. each voxel with intensity<theshold will be ignored in the statistics.

* this is because of the yolk which always gives a signal in DAPI channel and thereby will be treated as nuclei. this dramatically reduces

* the average intensity of somatic pCTD. since there is no pCTD signal in the yolk ignoring all voxels below threshold solves this issue. */

\section{for $(\mathrm{k}=$ thresholdpCTD; $\mathrm{k}<256 ; \mathrm{k}++)$ \{}

//ignore all values below threshold

$$
\text { if (measureSoma }==\text { true) }\{
$$

SomaNotUnder[k];

NumberSomaNotUnder $=$ NumberSomaNotUnder +

SomaUnder[k];

$(\mathrm{k} *$ SomaNotUnder[k]);

$$
\text { NumberSomaUnder }=\text { NumberSomaUnder }+
$$

TotalSomaNotUnder $=$ TotalSomaNotUnder +

TotalSomaUnder $=$ TotalSomaUnder $+(\mathrm{k} *$ SomaUnder $[\mathrm{k}])$

NumberVoxelsIn = NumberVoxelsIn + HistoIn[k];

//sum up all counts from histogram arrays

NumberVoxelsOut $=$ NumberVoxelsOut + HistoOut $[\mathrm{k}]$;

TotalValueIn $=$ TotalValueIn $+(\mathrm{k} *$ HistoIn $[\mathrm{k}])$;

//sum all

values(=intensities) from histogram arrays

TotalValueOut $=$ TotalValueOut $+(\mathrm{k} *$ HistoOut $[\mathrm{k}]) ;$

//calculate mean values

\}

MeanIntensityIn = TotalValueIn/NumberVoxelsIn;

MeanIntensityOut $=$ TotalValueOut/NumberVoxelsOut;

MeanSomaNotUnder $=$ TotalSomaNotUnder/NumberSomaNotUnder;

MeanSomaUnder $=$ TotalSomaUnder/NumberSomaUnder;

if $(\mathrm{i}==0)\{$

run("New... ", "name=NakedResults type=Table width=950

height=400"); //create new table if loop runs the first time print("[NakedResults]",

"I|Headings:stageltNumberVoxelsIn $\mid t$ TotalValueInltNumberVoxelsOutltTotalValueOutltMeanIntensityIn\tMeanInten 
sityOutltMeanSomaNotUnderltMeanSomaUnderltslideID ItnameltcommentltthresholdDNAltthresholdVasaltthreshold pCTDltyborder");

\} slideID = substring(filename, 0.12$) ; \quad$ //get info about staining from

filename length = lengthOf(filename); name $=$ substring(filename, $13,($ length- 4$)) ; \quad / /$ store results and file info in

table

stage $=$ substring(filename, 18,20$)$;

print("[NakedResults]", stage + "lt" + NumberVoxelsIn + "lt" + TotalValueIn

+ "It" + NumberVoxelsOut + "|t" + TotalValueOut + "lt" + MeanIntensityIn + "|t" + MeanIntensityOut + "lt"

+MeanSomaNotUnder+ "|t" +MeanSomaUnder+ "It" + slideID + "lt" + name + "lt" + comment+ "\t" + thresholdDNA

+ "lt" + thresholdVasa + "lt" + thresholdpCTD + "It" + lowerBorder);

\}

else $\{\quad / /$ is run if stack is skipped

$$
\text { if }(i==0)\{
$$

run("New... ", "name=NakedResults type=Table width=950

height=400"); $\quad$ //create new table if loop runs the first time print("[NakedResults]",

"|\Headings:stageltNumberVoxelsIn $\backslash t T o t a l V a l u e I n \backslash t N u m b e r V o x e l s O u t|t T o t a l V a l u e O u t| t M e a n I n t e n s i t y I n \backslash t M e a n I n t e n$ sityOutltMeanSomaNotUnderltMeanSomaUnderltslideID $\backslash$ tnameltcomment ttthresholdDNAltthresholdVasaltthreshold pCTDltyborder");

$$
\text { \} }
$$

slideID = substring(filename, 0.12$) ; \quad$ //get info about staining from

filename

$$
\text { length = lengthOf(filename); }
$$$$
\text { name = substring(filename,13,(length-4)); //insert empty line in results }
$$

table, just file info and "skipped"+commment

stage $=$ substring (filename, 18,20$)$;

print("[NakedResults]", stage + "|t" + "|t" + "|t" + "lt" + "|t" + "|t" + "|t" + "|t"

+ "It" + slideID + "It" + name + "It" + comment+ "It" + thresholdDNA + "It" + thresholdVasa + "It" + thresholdpCTD $+" \mid \mathrm{t}$ + lowerBorder);

\}

selectWindow("NakedResults");

saveAs("Text", dirSource+"NakedResults.xls");

selectWindow("Results");

run("Close");

setBatchMode(false);

\}

waitForUser("Info", "Naked results table saved in source folder");

\title{
Somatic vs. PGC distribution of osk and pgc RNA
}

\section{first macro}

\author{
//written by Andres Hertel March 2016 \\ macro "soma_vs_PGC_get-ROI" \{ \\ //folder must contain a subfolder linput. subfolder \temp will be created \\ dir = getString("Enter directory path: ", "path"); \\ setBatchMode(true); \\ numberChan = getNumber("Enter number of channels", 4); \\ //prompt user for channel identity. DNA, Vasa, measureThis (FISH), ignore \\ choice = newArray("DNA", "Vasa", "FISH", "ignore"); \\ Dialog.create("Specify channels"); \\ for (ch=0; $\mathrm{ch}<$ numberChan; $\mathrm{ch}++) / /$ variable "ch" is index of channel \\ Dialog.addChoice("Channel "+(ch+1)+":", choice); \\ chan = newArray(numberChan); \\ for $(\mathrm{ch}=0 ; \mathrm{ch}<$ numberChan; $\mathrm{ch}++)\{$
}


\}

chan[ch] $=$ Dialog.getChoice(); //save channel identity as array chan[ch]

//store list of files in source folder as array list[i]

dirSource = dir+"|linput I";

dirTemp = dir+"||templ|";

File.makeDirectory(dirTemp);

list $=$ getFileList $($ dirSource $)$;

for (file $=0$; file $<$ list.length; file ++ ) $\{$ //variable "file" is index of file list in input folder

filename $=$ list[file]; //read filename

ID = substring(filename, 0.15 ); //ID of the file. used for saving ROIs and other output

"st04". if not, skip that file if ((indexOf(filename, "st04")) != (-1)) \{ //check filename for presense for

open(dirSource+list[file]);

rename("bild"); //this makes it easier (while coding) since every file has the same name

now. real name is stored in "filename" variable run("Split Channels");

//rename channels according to input. close all ignore channels for (ch=0; ch<numberChan; ch++) \{

selectWindow("C"+(ch+1)+"-bild");

if $(\mathrm{chan}[\mathrm{ch}]==$ "ignore")

else close();

\} rename("bild-"+chan[ch]);

//get ROIs from nuclei. save ROI lists in somewhere.

selectWindow("bild-DNA");

runMacro("F:IIGWDG-

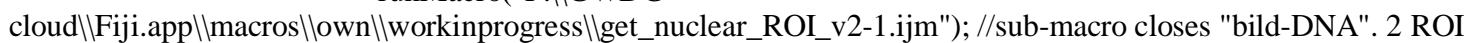

files are saved.

//rename ROI file from "bild-ROI" to "ID_ROI". ID is part of filename.

File.copy("F:IIsoma_vs_PGCIItempllbild_DNA-ROI.zip", dir+"II"+ID+"_DNA-

ROI.zip");

// File.copy("F:Ilsoma_vs_PGCIItest|lbild_DNA-ROI-spheres.zip",

"F:Ilsoma_vs_PGCl|test|ltempl|"+ID+"_DNA-ROI-spheres.zip")

dir+"|।"+ID+"_DNA-ROI-spheres.zip");

File.copy("F:Ilsoma_vs_PGClItempllbild_DNA-ROI-spheres.zip",

selectWindow("bild-Vasa");

//submacro for finding the PGCs. needs stack "bild-Vasa" and Roi file "bild_DNA-ROI-spheres.zip". saves a ROI file in folder.

runMacro("F:IIGWDG-

cloud\Fiji.app \|macros\lown》|workinprogress $\backslash$ identify_PGCs_and_label.ijm");

//copy ROI file

labeled.zip");

File.copy("F:Ilsoma_vs_PGC||temp|lbild_nuclei-labeled.zip", dirTemp+ID+"_nuclei-

//save vasa channel in temp folder. apply "fire" LUT first

selectWindow("bild-Vasa");

run("Fire");

saveAs("Tiff", dirTemp+ID+"-Vasa.tif");

close("*"); //close all image windows

run("Collect Garbage"); //run Java garbage collector, which flushes memory to free

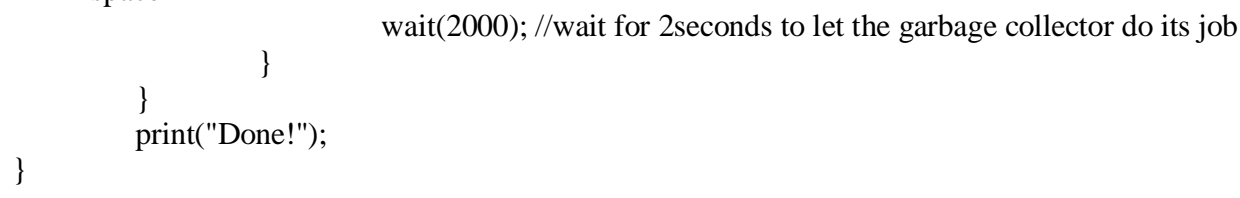

\section{$\underline{\text { second macro }}$}

//written by Andres Hertel February 2016

macro "get_nuclear_ROI" \{

$/ /$ macro should work on single channel stacks which contain the DAPI signal. make sure only one suitable image is open/active 
//image must be named "bild-DNA"

rename("bild-DNA");

getDimensions(width, height, channels, slices, frames);

//correct brightness/contrast in z-dimension. increases brightness depending on z

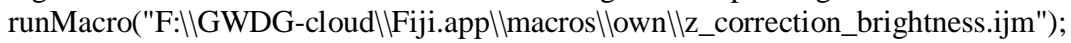

//remove background. threshold 30.

setMinAndMax(30. 255);

run("Apply LUT", "stack");

//gaussian smooth

run("Gaussian Blur...", "sigma=2 stack");

setMinAndMax(10. 255); //this reduces background substantially!!

run("Apply LUT", "stack");

//apply s-log curve. slope is set to 7

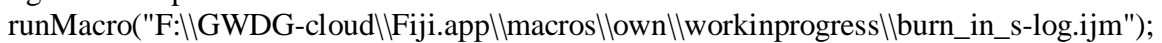

//find local maxima. use 3D suite plugin. radius in xyz is 3microns. use Voxelsize to calc pixel values

getVoxelSize(x,y,z,unit);

setVoxelSize(x,x,z,unit);

getVoxelSize(x,y,z,unit);

Voxelvolume $=\mathrm{x}^{*} \mathrm{y}^{*} \mathrm{z} ; \quad / /$ volume of one voxel in cubic microns

$\mathrm{x} \_$rad $=\operatorname{round}(3 / \mathrm{x})$;

y_rad $=\operatorname{round}(3 / \mathrm{y})$;

Z_rad $=\operatorname{round}(3 / \mathrm{z})$;

run("3D Fast Filters","filter=MaximumLocal radius_x_pix="+x_rad+" radius_y_pix="+y_rad+"

radius_z_pix $="+\mathrm{z} \_$rad+" Nb_cpus $=8 "$ );

//use "3D_MaximumLocal" as a seed for 3D watershed

run("3D Watershed", "seeds_threshold=50 image_threshold=0 image=bild-DNA

seeds=3D_MaximumLocal radius=2");

//get ROIs from watershed into 3D ROI manager

run("3D Manager");

selectWindow("watershed");

//save ROI list

Ext.Manager3D_AddImage();

Ext.Manager3D_Save("F:IIsoma_vs_PGCIItempllbild_DNA-ROI.zip");

close("bild-DNA");

close("3D_MaximumLocal");

close("watershed");//all image windows(from this sub macro) closed

//remove all ROIs with volume lower than 25 cubic microns (normal nucleus has around 60). they are noise.

counter $=0 ; \quad$ //object number is changing when an object is deleted. therefore manual increment of select-object-counter do \{

Ext.Manager3D_Select(counter);

Ext.Manager3D_Measure3D(counter,"Vol",volume);

if (volume $<(25 /$ Voxelvolume $))\{$

\} else \{ Ext.Manager3D_Delete();

\}

counter++;

Ext.Manager3D_Count(nb_obj);

\} while (counter $<$ nb_obj);

//get centroids from objects (nuclei) in 3D manager. draw sphere around center with $\mathrm{r}=5$ microns (use pixelvalues here!)

//then add image with sphere to 3D manager. close image. repeat for all objects.

Ext.Manager3D_Count(nb_obj); //nb_obj = number of objects in 3D manager (number of nuclei)

for $\left(\mathrm{m}=0 ; \mathrm{m}<\mathrm{nb} \_\mathrm{obj} ; \mathrm{m}++\right)\{$

Ext.Manager3D_Select(m); //m is current object

Ext.Manager3D_Centroid3D(m,cx,cy,cz);

run("3D Draw Shape", "size="+width+","+height+","+slices+"

center="+(round $(\mathrm{cx}))+", "+(\operatorname{round}(\mathrm{cy}))+",++(\operatorname{round}(\mathrm{cz}))+"$

radius="+(round $(5 / x))+",+(\operatorname{round}(5 / y))+", "+(\operatorname{round}(5 / z))+"$ vector1=1.0.0.0.0.0 vector2=0.0.1.0.0.0 res_xy=1.000

res_z $=1.000$ unit=unit value $=255$ display $=[$ New stack $] ")$;

selectWindow("Shape3D");

Ext.Manager3D_AddImage();

\} close("Shape3D"); 
//select first nb_obj objects, delete them. list now only contains ROIs defining spheres around each nucleus. save list Ext.Manager3D_SelectFor(0.(nb_obj),1);

Ext.Manager3D_Delete();

Ext.Manager3D_Save("F:I|soma_vs_PGC||temp|lbild_DNA-ROI-spheres.zip");

Ext.Manager3D_SelectAll();

Ext.Manager3D_Delete();

Ext.Manager3D_Close();

for $($ counter $=0$; counter $<3$; counter ++$)\{$ run("Collect Garbage");

\} wait(2000);

\}

\section{third macro}

//written by Andres Hertel March 2016

macro "identify PGCs" \{

//submacro for "soma_vs_PGC"

//requires single channel stack named "bild-Vasa" and a ROI list (from get_nuclear_ROI macro) setMinAndMax $(5,255)$;

run("Apply LUT", "stack"); //remove everything darker than 5. its background run("3D Manager");

//load ROI list which contains spheres around nuclei

Ext.Manager3D_Load("F:Isoma_vs_PGCIItempllbild_DNA-ROI-spheres.zip");

Ext.Manager3D_Count(nb_obj); //get number of object in list

Labels = newArray(nb_obj);

MeanValues = newArray $($ nb_obj);

LabelsSort = newArray(nb_obj);

MeanValuesSort = newArray $($ nb_obj $)$;

ratio $=$ newArray $($ nb_obj);

ratio[0] $=1$;

for $(o b j=0$; obj<nb_obj; obj++) \{

Ext.Manager3D_GetName(obj, objLabel);

Ext.Manager3D_Quantif3D(obj,"Mean",mean);

Labels[obj] = objLabel; //save labels and mean values as array

\} MeanValues[obj] = mean

ranks = Array.rankPositions(MeanValues); //get ranks of meanValues in anscending order

//sort Labels[obj] and MeanValues[obj] according to ranks. save as new arrays

for $\left(o b j=0 ; o b j<n b \_o b j ; o b j++\right)\{$

index $=$ ranks $[\mathrm{obj}]$;

LabelsSort[obj] = Labels[index $]$

\}

MeanValuesSort [obj] = MeanValues[index $]$;

//calculate relative change from MeanvaluesSort[obj] to next number in array for (obj=1;obj<nb_obj; obj++) //skip first entry as there is no previous one ratio[obj] = MeanValuesSort[obj]/MeanValuesSort[(obj-1)]; maxima $=$ Array.findMaxima $($ ratio, 0.01$)$;

//find the highest maximum for which MeanValue>5 (below that cannot be a PGC)

$\mathrm{obj}=0$;

do \{

index = maxima[obj];

obj++;

\} while $(($ MeanValuesSort[index]) < 5); //variable "index" now contains the position of first PGC in

MeanValuesSort[obj]

//every object equal or brighter than "MeanValuesSort[index]" is considered a PGC nucleus.

Ext.Manager3D_DeselectAll();

//rename all objects to "soma_<number>" or "PGC_<number>"

for $\left(o b j=0 ; o b j<n b \_o b j ; o b j++\right)\{$

Ext.Manager3D_Select(obj);

if $(($ MeanValues [obj] $)<($ MeanValuesSort[index] $))$

else

Ext.Manager3D_Rename("soma_"+obj);

Ext.Manager3D_Rename("PGC_"+obj); 
\}

Ext.Manager3D_SelectAll();

//save selected objects as file

Ext.Manager3D_Save("F:Issoma_vs_PGC||temp|lbild_nuclei-labeled.zip");

//delete all objects, then close 3D manager, then run garbage collector 3 times

Ext.Manager3D_Delete();

Ext.Manager3D_Close();

for $($ counter $=0$; counter $<3$; counter++) \{ run("Collect Garbage");

\} wait(2000);

\}

\section{fourth macro}

//written by Andres Hertel February-June 2016

macro "soma_vs_PGC_measure" \{

dir = getString("Enter directory path: ", "path");

setBatchMode(true);

numberChan = getNumber("Enter number of channels", 4);

//ask for number of channels

//prompt user for channel identity. DNA, Vasa, measureThis (FISH), ignore

choice = newArray("DNA", "Vasa", "FISH", "ignore");

Dialog.create("Specify channels");

for (ch=0; ch<numberChan; ch++) //variable "ch" is index of channel

Dialog.show(); Dialog.addChoice("Channel "+(ch+1)+":", choice);

chan = newArray (numberChan);

for $(\mathrm{ch}=0$; $\mathrm{ch}<$ numberChan; $\mathrm{ch}++)$ \{

\}

chan $[\mathrm{ch}]=$ Dialog.getChoice();//save channel identity as array chan[ch]

threshVASA $=10$; //threshold for quantifying Vasa staining later. every voxel below threshVASA will be

ignored

threshFISH $=10 ;$ //threshold for quantifying FISH staining later. every voxel below threshFISH will be

ignored

dirSource = dir+"|linputll";

dirTemp = dir+"||templ|",

list = getFileList(dirSource); //store list of files in source folder as array list[i]

$/ /$ create output table

tableName $=$ "[results_table]";

run("New... ", "name="+tableName+" type=Table"); print(tableName,

"IlHeadings:filenameltnb_PGCltVASAVasaMaskOUTvoxltVASAVasaMaskOUTtotltVASAVasaMaskINvox ltVAS AVasaMaskINtotltFISHVasaMaskOUTvox $t$ tFISHVasaMaskOUTtotltFISHVasaMaskINvox ltFISHVasaMaskINtot $\backslash t$ VASAPGCMaskOUTvox $|t V A S A P G C M a s k O U T t o t| t V A S A P G C M a s k I N v o x l t V A S A P G C M a s k I N t o t \mid t F I S H P G C M a s$ kOUTvoxltFISHPGCMaskOUTtotltFISHPGCMaskINvox ltFISHPGCMaskINtotltcomments"); //header of the table for (file $=0$; file $<$ list.length; file ++$)\{$ //variable "file" is index of file list in input folder filename = list[file]; //read filename $\mathrm{ID}=$ substring(filename, 0.15$)$; //ID of the file. used for saving ROIs and other output

"st04". if not, skip that file if ((indexOf(filename, "st04")) != (-1)) \{ //check filename for presense for

open(dirSource+list[file]);

rename("bild"); //this makes it easier (while coding) since every file has the same name

now. real name is stored in "filename" variable getVoxelSize(x,y,z,unit); run("Split Channels");

//rename channels according to input. close all ignore channels for $(\mathrm{ch}=0$; $\mathrm{ch}<$ numberChan; $\mathrm{ch}++)\{$ selectWindow("C"+(ch+1)+"-bild");

if $(\mathrm{chan}[\mathrm{ch}]==$ "ignore") close ();

else

rename("bild-"+chan[ch]);

\}

//create mask from Vasa channel. duplicate image first.

selectWindow("bild-Vasa"); 
run("Select None"); //make sure there is no selection in image! f*cking important!

run("Duplicate...", "duplicate");

selectWindow("bild-Vasa-1");

rename("bild-Vasa-mask");

run("Gaussian Blur...", "sigma=0.2 scaled stack");

setMinAndMax(10. 255); //remove background

run("Apply LUT", "stack");

//first blur, then background removal is good for getting rid of unspecific stuff

run("Gaussian Blur...", "sigma=0.2 scaled stack");

setMinAndMax (5, 255); //remove background

run("Apply LUT", "stack");

$\log . \mathrm{ijm} ")$

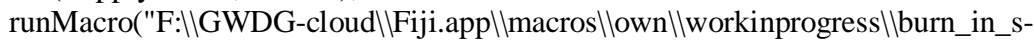

run("Gaussian Blur...", "sigma=0.50 scaled stack");

setMinAndMax(20. 255); //remove background

run("Apply LUT", "stack");

//mask is ready for removal of somatic Vasa

run("3D Manager");

contains somatic spheres (manually corrected)

Ext.Manager3D_Load(dirTemp+ID+"_nuclei-labeled.zip"); //load ROI file which

selectWindow("bild-Vasa-mask");

Ext.Manager3D_SelectAll();

Ext.Manager3D_Erase(); //deletes objects from list AND deletes regions in the stack

setThreshold(1, 255);

run("Make Binary", "method=Default background=Default black");

Ext.Manager3D_AddImage(); //add remainings as ROI, then save it

Ext.Manager3D_Save(dir+"||"+ID+"_Vasa-clean.zip");

Ext.Manager3D_SelectAll();

Ext.Manager3D_Delete();

Ext.Manager3D_Close();

//done with making Vasa mask. proceed with making mask from PGCs

selectWindow("bild-FISH");

getDimensions(width, height, channels, slices, frames); //get info about image size and

slices

newImage("HyperStack", "8-bit color-mode", width, height, 1, slices, 1); //create blank

(black) stack with same size. there the ROIs will be drawn

run("3D Manager");

Ext.Manager3D_Load(dir+"||"+ID+"_DNA-ROI-spheres.zip"); //load ROI file with

nuclear spheres (all nuclei!)

/l

Ext.Manager3D_Count(nb_nuc); //number of objects is number of nuclei

print("nb_nuc: "+nb_nuc);

selectWindow("HyperStack");

Ext.Manager3D_SelectAll(); //select all objects

Ext.Manager3D_FillStack(255, 255, 255); //fills the ROI with white

Ext.Manager3D_Delete(); //deletes objects from ROI manager list

//HyperStack now has all nuclear spheres filled

Ext.Manager3D_Load(dirTemp+ID+"_nuclei-labeled.zip"); //load ROI file which contains somatic spheres (manually corrected)

Ext.Manager3D_Count(nb_som);

nb_PGC = (nb_nuc - nb_som); //number of PGC!

Ext.Manager3D_SelectAll(); //select all objects

Ext.Manager3D_Erase(); //deletes objects from list AND deletes regions in the stack

//HyperStack now only contains PGC spheres

Ext.Manager3D_Close();

//expand PGC selection in 3D. 1micron in all directions.

//ratio of xy-scale to z-scale determines how often "dilate" (only xy-dimension) is used before using "dilate 3D" (xyz) once

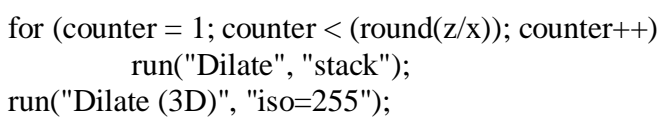

//load soma-spheres ROI file. then delete these regions in "hyperStack". whats left are PGCs without somatic Vasa/FISH signal!!

$$
\text { run("3D Manager"); }
$$


contains somatic spheres (manually corrected)

Ext.Manager3D_Load(dirTemp+ID+"_nuclei-labeled.zip"); //load ROI file which

Ext.Manager3D_SelectAll(); //select all objects

Ext.Manager3D_Erase(); //deletes objects from list AND deletes regions in the stack

Ext.Manager3D_AddImage(); //add remainings as ROI, then save it

Ext.Manager3D_Save(dir+"|।"+ID+"_PGC-clean.zip");

Ext.Manager3D_SelectAll();

Ext.Manager3D_Delete();

Ext.Manager3D_Close();

//done with making PGC mask. proceed with measuring

/* use both masks (from Vasa channel: "bild-Vasa-mask" and from PGCs: "HyperStack") to measure intensities inside and outside these

masks. measure in Vasa channel and in FISH channel. so 8 measurements: two channels, two masks, inside \& outside. $(2 * 2 * 2=8)$

for each measurement the number of voxels above threshold and the total intensity of these voxels are stored. (= 16 values)

mean values can be calculated later in excel/origin if needed. names of variables are:

VASAVasaMaskOUTvox, VASAVasaMaskOUTtot, VASAVasaMaskINvox, VASAVasaMaskINtot

FISHVasaMaskOUTvox, FISHVasaMaskOUTtot, FISHVasaMaskINvox, FISHVasaMaskINtot

VASAPGCMaskOUTvox, VASAPGCMaskOUTtot, VASAPGCMaskINvox, VASAPGCMaskINtot

FISHPGCMaskOUTvox, FISHPGCMaskOUTtot, FISHPGCMaskINvox, FISHPGCMaskINtot

$* /$

histograms

VASAVasaMaskOUT $=$ new $\operatorname{Array}(256)$

VASAVasaMaskIN = newArray $(256)$;

FISHVasaMaskOUT = newArray(256);

FISHVasaMaskIN = newArray(256);

VASAPGCMaskOUT = newArray(256);

VASAPGCMaskIN = newArray (256);

FISHPGCMaskOUT = newArray(256);

FISHPGCMaskIN = newArray (256);

//use Vasa-mask first.

for $(j=0 ; j<$ slices $; j++)\{$

selectWindow("bild-Vasa-mask");

setSlice $(\mathrm{j}+1)$;

run("Create Selection");

if (selectionType != -1) \{ //skip slice if nothing selected

selectWindow("bild-Vasa");

setSlice $(j+1)$;

run("Restore Selection");

getHistogram(values, counts, 256);

for $(\mathrm{k}=0 ; \mathrm{k}<256 ; \mathrm{k}++)$

counts[k]; $\quad$ //sum up histogram counts and store as array

VASAVasaMaskOUT[k] = VASAVasaMaskOUT[k] +

run("Make Inverse");

getHistogram(values, counts, 256);

for $(\mathrm{k}=0 ; \mathrm{k}<256$; $\mathrm{k}++)$

VASAVasaMaskIN $[\mathrm{k}]=$ VASAVasaMaskIN $[\mathrm{k}]+$

counts[k]; $\quad$ //sum up histogram counts and store as array

selectWindow("bild-FISH"); //repeat measuring in FISH channel setSlice $(j+1)$;

run("Restore Selection");

getHistogram(values, counts, 256);

for $(\mathrm{k}=0 ; \mathrm{k}<256 ; \mathrm{k}++)$

FISHVasaMaskIN $[\mathrm{k}]$ = FISHVasaMaskIN $[\mathrm{k}]+\operatorname{counts}[\mathrm{k}]$;

//sum up histogram counts and store as array

run("Make Inverse");

getHistogram(values, counts, 256);

for $(\mathrm{k}=0 ; \mathrm{k}<256 ; \mathrm{k}++)$ FISHVasaMaskOUT[k] = FISHVasaMaskOUT[k] +

counts[k];

//sum up histogram counts and store as array

\}

\}

//now repeat all the measuring with PGCmask 


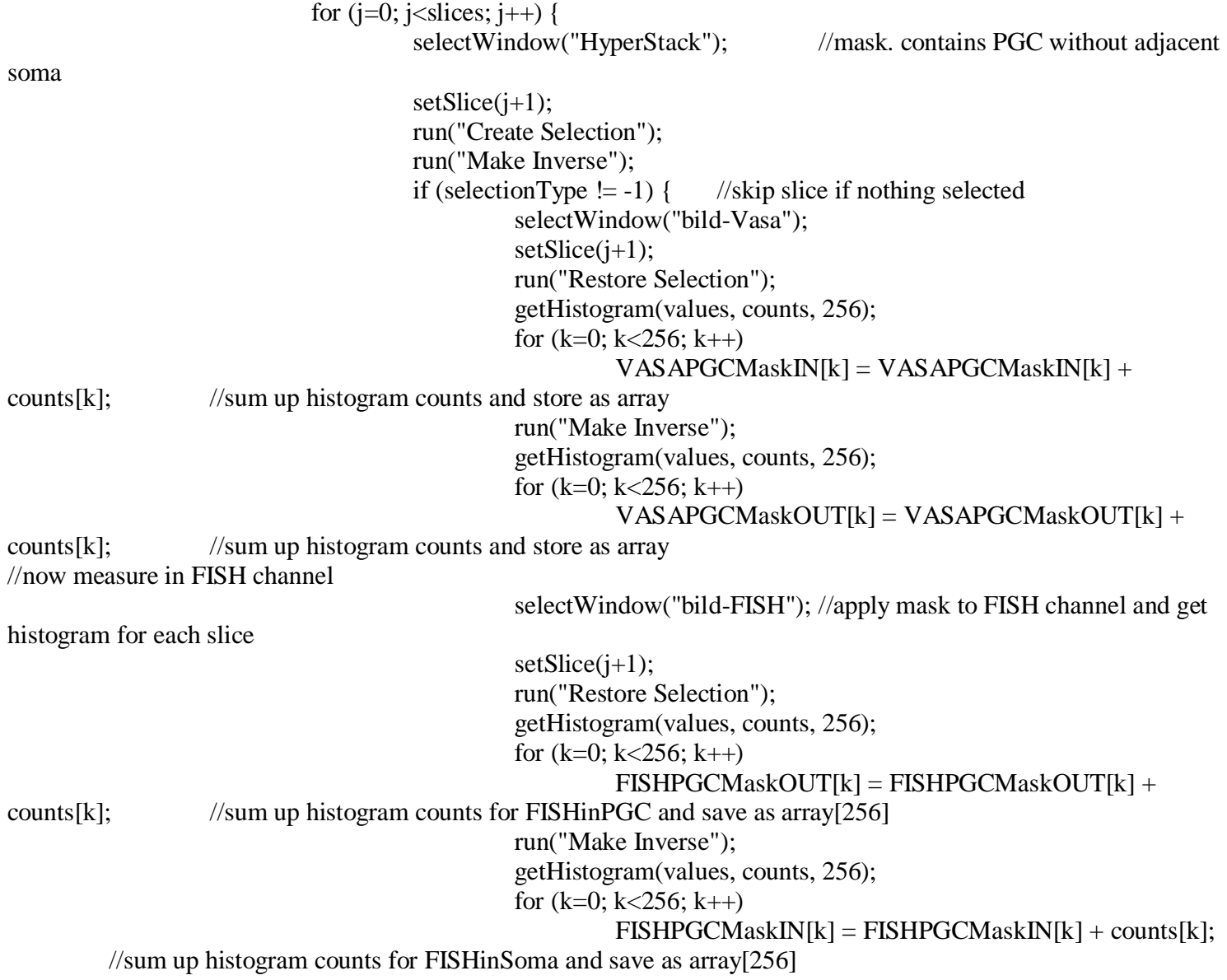




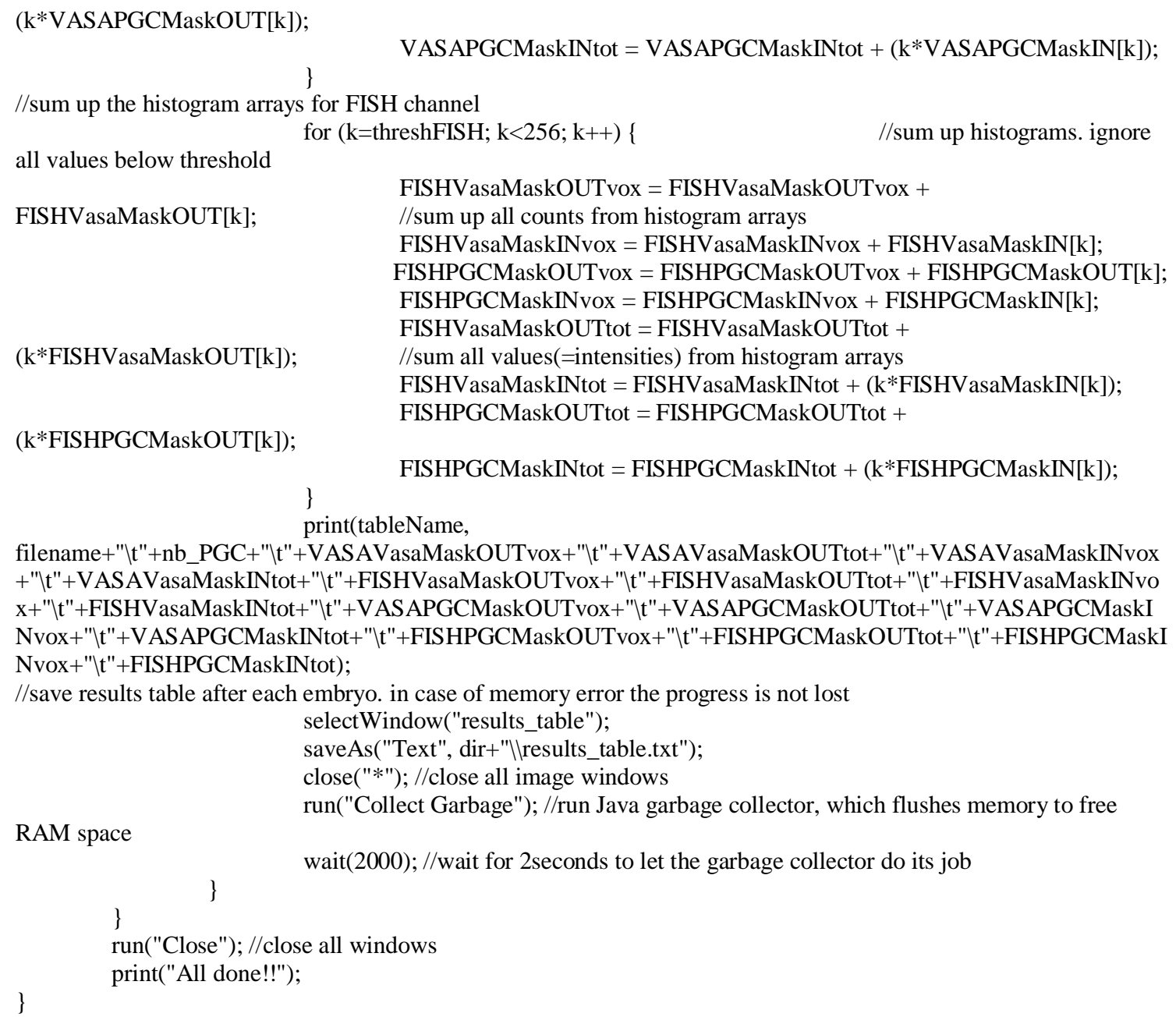

\section{Germ plasm intensity profiles}

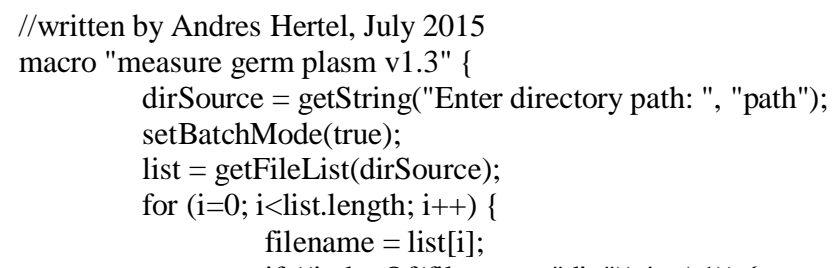

wrong orientation

rename("bild");

run("Rotate 90 Degrees Right");

slices $=$ nSlices $/ 4$

//divide by number of channels

run("Make Substack...", "channels=3-4 slices=1-"+slices+""); //keep two

channels open, but measure only one. or else "enhance contrast" isn't working

run("Split Channels");

selectWindow("C1-bild-1"); $\quad$ //C1 = VASA channel; $\mathrm{C} 2$ = pgc channel

run("Enhance Contrast...", "saturated=0.001 process_all use"); //(at least) two

channels have to be open to perform this task. one is not working! 


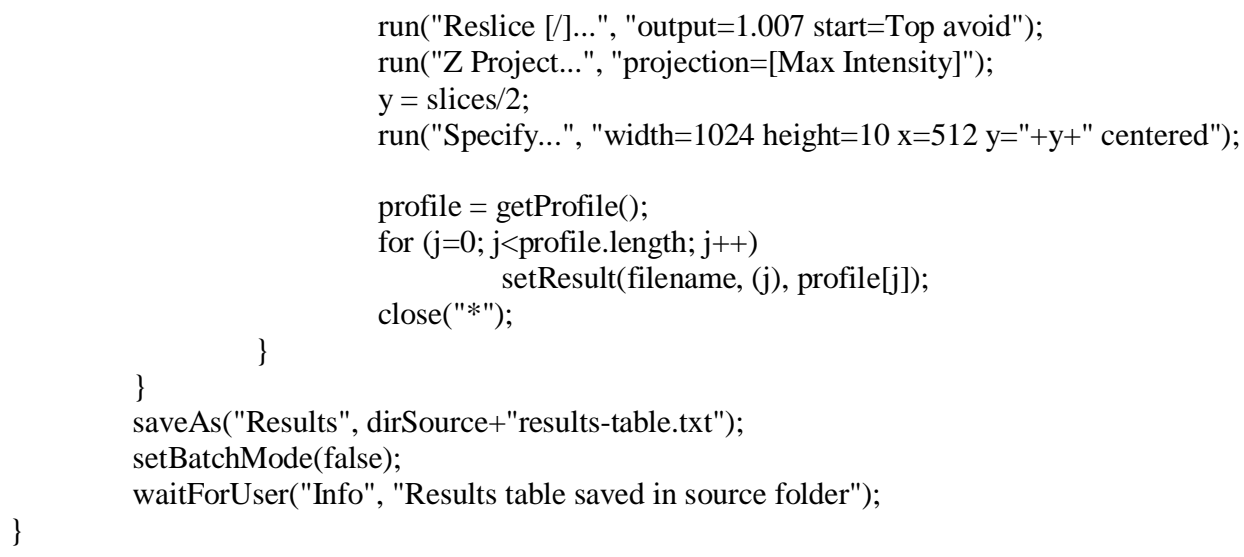

\section{Others}

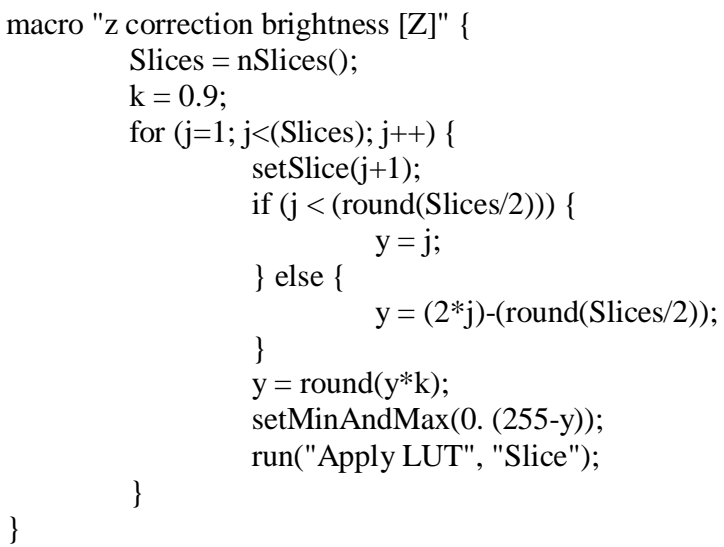

//written by Andres Hertel, October 2015

macro "apply s-log"

setBatchMode(true);

//defines slope of log-function. 1 means almost linear. the higher the value the steeper the slope. must be integer

slope $=7$

getDimensions(width, height, channels, slices, frames);

if (channels > 1)

exit("Single channel image required!");

//determine brightest pixel in stack, store in maxVal variable

$\max \mathrm{Val}=0$;

for $(\mathrm{i}=0 ; \mathrm{i}<$ slices; $\mathrm{i}++)\{$

setSlice $(\mathrm{i}+1)$;

getStatistics(area, mean, min, max);

if $(\max >\max V a l)$

$$
\max V a l=\max
$$

\}

//find real bit depth of picture. the smallest $\mathrm{n}$ with $2^{\wedge} \mathrm{n}>\max V a \mathrm{l}$

bit $=0$;

do \{

bit++;

\} while $(((\operatorname{pow}(2$, bit $))-1)<\max V a l)$;

if (bit $<8$ )

bit $=8 ; \quad / /$ minimum depth is 8 bit

//increase brightness of stack that it uses complete dynamic range of $\left(2^{\wedge}\right.$ bit $)-1$

factor $=((\operatorname{pow}(2$, bit $))-1) / \mathrm{max} V a \mathrm{l}$;

for $(i=0 ; i<$ slices; $i++)\{$ 
setSlice $(\mathrm{i}+1)$;

for $(\mathrm{x}=0 ; \mathrm{x}<$ width; $\mathrm{x}++)\{$

for $(y=0 ; y<$ height; $y++)\{$

intensity $=\operatorname{getPixel}(\mathrm{x}, \mathrm{y})$

intensity $=$ floor(intensity*factor $)$;

\} setPixel(x,y,intensity);

\}

//calculate variables for log-function

factor $=\operatorname{pow}(2,($ bit-slope $)$;

correction $=1-(1 /(2 *$ factor $))$;

if $($ factor $<1)$

correction $=0$;

scaling $=((\operatorname{pow}(2$, slope $)) /(\log (\operatorname{pow}(2$, slope $)))) *$ factor;

scalingCorrection $=\log (((\operatorname{pow}(2$, bit $))-1) /$ factor $) / \log (((\operatorname{pow}(2$, bit $))-1) /$ factor $)+$ correction $)$;

//apply log-function to every pixel in every slice

for $(\mathrm{i}=0 ; \mathrm{i}<$ slices; $\mathrm{i}++)$

setSlice $(\mathrm{i}+1)$;

for $(\mathrm{x}=0 ; \mathrm{x}<$ width; $\mathrm{x}++)\{$

for $(y=0 ; y<$ height; $y++)\{$

intensity $=$ getPixel $(\mathrm{x}, \mathrm{y})$

if (intensity $!=0)\{$

intensity $=$

$\log (($ intensity/factor $)+$ correction $) *$ scaling*scalingCorrection;

intensity = floor(intensity);

setPixel(x,y,intensity);

run("Collect Garbage"); 